AcCePted for Publication in The Astrophysical Journal

Preprint typeset using $\mathrm{LAT}_{\mathrm{E}} \mathrm{X}$ style emulateapj v. 05/12/14

\title{
BRIGHTEST CLUSTER GALAXIES AT THE PRESENT EPOCH
}

\author{
TOD R. LAUER \\ National Optical Astronomy Observatory, ${ }^{*}$ P.O. Box 26732, Tucson, AZ 85726 \\ Marc Postman \\ Space Telescope Science Institute, ${ }^{\dagger} 3700$ San Martin Drive, Baltimore, MD 21218
}

\author{
Michael A. Strauss, Genevieve J. Graves, and Nora E. Chisari \\ Department of Astrophysical Sciences, Princeton University, Princeton, NJ \\ Accepted for Publication in The Astrophysical Journal
}

\begin{abstract}
We have obtained photometry and spectroscopy of $433 z \leq 0.08$ brightest cluster galaxies (BCGs) in a full-sky survey of Abell clusters to construct a BCG sample suitable for probing deviations from the local Hubble flow. The BCG Hubble diagram over $0<z<0.08$ is consistent to within $2 \%$ of the Hubble relation specified by a $\Omega_{m}=0.3, \Lambda=0.7$ cosmology. This sample allows us to explore the structural and photometric properties of BCGs at the present epoch, their location in their hosting galaxy clusters, and the effects of the cluster environment on their structure and evolution. We revisit the $L_{m}-\alpha$ relation for BCGs, which uses $\alpha$, the log-slope of the BCG photometric curve of growth, to predict the metric luminosity in an aperture with $14.3 \mathrm{kpc}$ radius, $L_{m}$, for use as a distance indicator. Residuals in the relation are $0.27 \mathrm{mag} \mathrm{rms}$. We measure central stellar velocity dispersions, $\sigma$, of the BCGs, finding the Faber-Jackson relation to flatten as the metric aperture grows to include an increasing fraction of the total BCG luminosity. A 3-parameter "metric plane" relation using $\alpha$ and $\sigma$ together gives the best prediction of $L_{m}$, with $0.21 \mathrm{mag}$ residuals. The distribution of projected spatial offsets, $r_{x}$ of BCGs from the X-ray-defined cluster center is a steep $\gamma=-2.33$ power-law over $1<r_{x}<10^{3} \mathrm{kpc}$. The median offset is $\sim 10 \mathrm{kpc}$, but $\sim 15 \%$ of the BCGs have $r_{x}>100 \mathrm{kpc}$. The absolute cluster-dispersion normalized BCG peculiar velocity $\left|\Delta V_{1}\right| / \sigma_{c}$ follows an exponential distribution with scale length $0.39 \pm 0.03$. Both $L_{m}$ and $\alpha$ increase with $\sigma_{c}$. The $\alpha$ parameter is further moderated by both the spatial and velocity offset from the cluster center, with larger $\alpha$ correlated with the proximity of the BCG to the cluster mean velocity or potential center. At the same time, position in the cluster has little effect on $L_{m}$. Likewise, residuals from the metric plane show no correlation with either the spatial or velocity offset from the cluster center. The luminosity difference between the BCG and second-ranked galaxy, M2, increases as the peculiar velocity of the BCG within the cluster decreases. Further, when M2 is a close luminosity "rival" of the BCG, the galaxy that is closest to either the velocity or X-ray center of the cluster is most likely to have the larger $\alpha$. We conclude that the inner portions of the BCGs are formed outside the cluster, but interactions in the heart of the galaxy cluster grow and extend the envelopes of the BCGs.

Keywords: galaxies: clusters: general — galaxies: distances and redshifts — galaxies: elliptical and lenticular, $\mathrm{cD}$ - galaxies: fundamental parameters - galaxies: photometry
\end{abstract}

\section{THE MOST MASSIVE GALAXIES IN THE UNIVERSE}

The brightest and most massive galaxies in the presentday Universe are the first-ranked or brightest cluster galaxies $(\mathrm{BCG})$ in rich galaxy clusters. The first studies of BCGs focussed on their high and almost "standardcandle" luminosities, which allowed the Hubble-flow to be characterized out to large distances (Sandage 1972a b. Gunn \& Oke 1975). The dispersion about the mean luminosity was shown to be significantly smaller than would be the case had the BCGs simply been the brightest galaxies drawn from a standard luminosity function

*The National Optical Astronomy Observatory is operated by AURA, Inc., under cooperative agreement with the National Science Foundation.

$\dagger$ Operated by the Association of Universities for Research in Astronomy, Inc., for the National Aeronautics and Space Administration.
(Tremaine \& Richstone 1977; Loh \& Strauss 2006). The narrowness of the BCG luminosity distribution does not extend to less massive galaxy groups (Geller \& Postman 1983), however; and more recent work argues that only the more luminous BCGs may be special (Lin et al. 2010). These results highlight the need to understand at what mass scale the unique formation and evolution mechanisms that shape BCGs come into play.

The acronym "BCG" underscores that these galaxies are tied to the galaxy clusters that host them. If BCGs are indeed special it is likely to be because their formation and evolution is tied to physical mechanisms unique to rich galaxy clusters. Cannibalism, whereby a BCG sitting in the middle of the cluster potential tends to engulf and merge with its neighbors, has been invoked to explain the high luminosity of these systems (e.g., Ostriker \& Tremaine 1975, Hausman \& Ostriker 1978, but see also Richstone 1975), but it remains unclear why this leads to 
such uniform properties, especially when we know that many clusters undergo interactions and merging. BCG growth by cannibalism does appear to take place in clusters at some level (Lauer 1988), however, dynamical arguments suggest that most of the BCG assembly takes place outside the cluster (Merritt 1985).

The properties of BCGs are distinct from those of the other galaxies in clusters, and any model for their formation has to acknowledge this. BCGs generally sit close to the X-ray centers of their hosting clusters and usually have small "peculiar" velocities relative to the cluster mean. Sastry (1968), Binggeli (1982), Lambas et al. (1988), and others showed that BCGs tend to be aligned with their parent cluster. This has been explored in detail with data from the SDSS by Niederste-Ostholt et al. (2010) and Hao et al. (2011), who found that this alignment is marked only in clusters in which the BCG is dominant, i.e., more than 0.65 mag brighter than the average of the second and third-ranked galaxies. Those clusters in which the BCG is not strongly dominant may be systems that recently underwent a merger, and are therefore not completely relaxed. In short, in many ways the BCG reflects the environment of the cluster that hosts it.

Our approach to understanding the origin of BCGs is to conduct an extensive examination of their presentday structure, luminosity, and cluster environments. We organize our thinking around three broad questions:

\subsection{What Are the Present-Day Properties of BCGs?}

The dispersion in the luminosities of the BCGs about the mean Hubble relation, measured by the first studies to use BCGs as distance indicators, was typically 0.3 to 0.4 magnitudes (Sandage 1972a b; Gunn \& Oke 1975). An important refinement of the use of BCG as distance indicators was developed by Hoessel (1980), who showed that BCG metric luminosity, $L_{m}$, was correlated with the logarithmic slope, $\alpha$, of the photometric curve-ofgrowth. The $L_{m}-\alpha$ relation is a form of a luminosityradius relation that side-steps the difficulties of characterizing the extended envelopes of BCG at large radii and faint isophotal levels. Postman \& Lauer 1995, hereafter $\mathrm{PL} 95)$ reinvestigated the use of $\mathrm{BCG}$ as distance indicators, using the $L_{m}-\alpha$ relation for a full-sky characterization of the linearity of the local Hubble-flow (Lauer \& Postman 1992) and providing a distant reference-frame to measure the relative peculiar velocity of the Local Group (Lauer \& Postman 1994). Residuals about the PL95 $L_{m}-\alpha$ relation were only $0.25 \mathrm{mag}(\mathrm{rms})$.

This paper presents a large full-sky sample of BCGs in Abell clusters over the redshift range $0<z \leq 0.08$. The original goal for obtaining this sample was to extend the bulk-flow analysis of Lauer \& Postman (1994) to greater distances. That work implied that the Abell clusters within $z \leq 0.05$ participated in a coherent motion in excess of $689 \pm 178 \mathrm{~km} \mathrm{~s}^{-1}$ superimposed on the background cosmological expansion or "Hubble flow" within the volume containing the sample. This analysis will be presented in a separate work. Requirements for measuring accurate bulk flows, however, specify much of the sample definition, observational methodologies, and analysis of the BCG properties undertaken in this work. A full sky sample allows for the optimal determination of any large-scale bulk mass flow. The relatively low redshiftlimit of the sample and its overall size is dictated by the scale out to which the BCGs can be used as accurate distance indicators. The observational methodology is driven by the need to obtain highly uniform photometry over the angular and spatial extent of the sample. Much of the analysis is a reinvestigation of the use of BCGs as distance indicators, with a substantially larger sample and new observations that go well beyond the material available to PL95.

Regardless of the bulk-flow analysis, the present sample offers an excellent opportunity to assess the structural properties of BCG to understand their origin, evolution over time, and their particular uniqueness as the luminous endpoint of galaxy formation, problems that were not addressed by the smaller sample and less-complete cluster information available to Postman \& Lauer (1995). Oegerle \& Hoessel (1991) and Lauer et al. (2007), for example, found that the central stellar velocity dispersions, $\sigma$, of BCGs increase very slowly if at all with the total BCG luminosity (also see Bernardi et al. 2007; von der Linden et al. 2007; Liu et al. 2008). 'Typical BCG $\sigma$ values are modest for their large luminosities, which may reflect the origin of BCGs in "dry" mergers (BoylanKolchin et al. 2006). In contrast, BCGs are unusually extended as compared to giant ellipticals, as is seen in the relation between effective radius, $R_{e}$, and total luminosity of the BCGs (Lauer et al. 2007; Bernardi et al. 2007). We will use the structure of BCGs as a probe of the effects of cluster environment on their evolution. In a companion paper (Chisari et al. 2014) we will compare the structure of BCGs to those of other highly luminous elliptical galaxies. The mutual relations between $L, \sigma$, and $R_{e}$ for elliptical galaxies overall are understood as various projections of the "fundamental plane" (Dressler et al. 1987; Djorgovski \& Davis 1987). Understanding the relationship of BCGs to the fundamental plane will be explored in Chisari et al. (2014).

\subsection{Where Are the BCGs Located in Their Galaxy Clusters?}

The "textbook" picture of a galaxy cluster is that it is a swarm of galaxies anchored by a massive $\mathrm{cD}$ residing at rest in the exact center of the potential as marked by hot, X-ray emitting, gas. Early work on the X-ray morphology of galaxy clusters (Jones \& Forman 1984) and their velocity structure (Quintana \& Lawrie 1982) indeed show that the BCG is likely to be centrally located. There are certainly examples of such clusters in our sample. At the same time, there are also massive galaxy clusters, like Coma (Abell 1656), in which neither the BCG, nor second-ranked galaxy, M2, are at the center of the potential. Coma may be the recent merger of two clusters, and this is the point - the position of the BCG with respect to the center of the potential, X-ray emission, may testify to the evolutionary state of both the BCG and the cluster. More recent work (Patel et al. 2006, Hashimoto et al. 2014) shows that the BCG is often displaced from the center of the cluster potential as defined by the X-ray emission. For the present sample of clusters we have quantified the distribution of projected spatial-offsets of the BCGs, finding that it is a steep power-law over three decades in radius.

For the BCG to reside at the spatial center of the cluster, it must also be at rest there. It has long been known that there are BCGs with "significant" peculiar veloci- 
ties within the cluster (Zabludoff et al. 1990, Malumuth 1992 Zabludoff et al. 1993 Oegerle \& Hill 2001). PL95 described the overall distribution of BCG peculiar velocities within their sample as a Gaussian with dispersion, $\sigma_{1}=264 \mathrm{~km} \mathrm{~s}^{-1}$, comparing this to the substantially larger mean cluster velocity-dispersion $\sigma_{c}=666 \mathrm{~km} \mathrm{~s}^{-1}$. We attempted to verify this result with our much larger present sample, finding now that the distribution of peculiar velocities is exponential, extending out to galaxies with $\Delta V_{1}>\sigma_{c}$.

Both the distributions of the spatial and velocity offsets of the BCGs are particularly interesting when compared to the Martel et al. (2014) simulations of galaxy cluster formation and evolution. These simulations emphasize that the location of the BCG within a cluster bears witness to its history of formation from smaller accreted groups and clusters. The dark matter, galaxy, and Xray-emitting gas distributions in any cluster all have different timescales and physical mechanisms for responding to the accretion or interaction with another cluster. The locations of the BCGs reflect this. Skibba et al. (2011) studied the peculiar velocities and spatial positions of a large sample of clusters and groups, showing that the location of the brightest galaxy in the systems provides a sharp test of the mechanisms that formed them.

\subsection{How Does the Cluster Environment Relate to the Properties of the BCGs?}

The relationship of the structure and luminosity of the BCG to the properties of the cluster has proven to be a multi-faceted problem. Initial work showed the BCG luminosity to be only weakly related to the richness of the clusters (Sandage 1972b, Sandage \& Hardy 1973; Sandage 1975, 1976). We re-investigated this relation in PL95, and saw no relation between the metric luminosity and cluster richness.

BCG luminosity and structure, however, do appear to be related to the X-ray properties of the clusters. Schombert (1988) found that the envelope luminosity of cD galaxies, a subset of the BCGs, increases with total cluster X-ray luminosity. Edge (1991) and Edge \& Stewart (1991) found a strong relationship between BCG luminosity and cluster X-ray temperature, which itself is closely related to the cluster velocity dispersion (Solinger \& Tucker 1972). Hudson \& Ebeling (1997) and Collins \& Mann (1998) also found that BCG luminosity increases with cluster X-ray luminosity. Lastly, Brough et al. (2005) found the structure of the BCG to also correlate with cluster X-ray luminosity, with the BCG envelope becoming more extended in more luminous clusters.

In this paper we show that both the BCG luminosity, and the radial extent (as characterized by $\alpha$ ) of their envelopes (where by "envelope" we mean simply the outer portions of the galaxies) correlate with cluster velocity dispersion. We take this a step further, however, finding that the extent of the envelope is related to both the spatial and velocity positions of the BCG within its hosting cluster. The luminosity and structural difference between the BCG and the second ranked galaxy, M2, also appears to depend on which galaxy has the smaller peculiar velocity within the cluster or the smaller offset from the center of the cluster potential as marked by X-ray emission. Beers \& Geller (1983) found that early type galaxies with extended halos (e.g., D or cD galaxies) lie on significant peaks in the cluster galaxy distribution regardless of whether they are the BCG. We now see how the structure of the BCG itself changes smoothly as a function of how close to the center of the cluster it resides.

\subsection{This Paper}

We begin in $\$ 2$ with the geometric and redshift selection of the Abell clusters defining the present sample, detailing the imaging observations used both to select the BCG for any given cluster and to provide accurate surface photometry. Spectroscopic observations are presented, which provide BCG redshifts and central stellar velocity dispersions. A crucial part of the sample definition is the derivation of accurate mean redshifts and velocity dispersions for the galaxy clusters. The projected spatial and velocity locations of the BCGs within their clusters is presented in 83 . The photometric and kinematic properties of the BCGs are presented in 84 , with particular attention to parametric relations between the metric luminosity and BCG structure. This section also explores the relationship between BCG properties and cluster environment. Additional information about the BCGs is provided by the properties of the second-ranked galaxies, M2, which are presented in \$5. We summarize what we have learned about the origin and evolution of BCGs in the final section of the paper.

\section{A FULL-SKY SAMPLE OF LOCAL BCGS 2.1. Definition of the Sample}

The present sample of BCGs comprises 433 Abell (1958) and ACO (Abell et al. 1989) galaxy clusters with mean heliocentric velocities, $V<24,000 \mathrm{~km} \mathrm{~s}^{-1}$ and galactic latitude, $|b| \geq 15^{\circ}$. There is no limit on the minimum richness class of the clusters. Table 1 lists the BCG coordinates, and heliocentric velocities, $V_{1}$, as well as the cluster velocities, $V_{c}$, cluster velocity dispersions, $\sigma_{c}$, the number of galaxy velocities used to compute these, $N_{g}$, and the Schlegel et al. (1998) $A_{B}$ values. The distribution on the sky in galactic coordinates is shown in Figure 1. We use a cosmological model with $H_{0}=70 \mathrm{~km} \mathrm{~s}^{-1} \mathrm{Mpc}^{-1}, \Omega_{m}=0.3$, and $\Lambda=0.7$ throughout this paper.

The sample was originally designed to serve as a reference frame to measure the peculiar velocity of the Milky Way. The inferred luminosities of the BCGs serve as distance indicators, following the methodology presented in Lauer \& Postman (1994, hereafter LP94). In that paper a volume-limited frame was constructed from 119 clusters with $V<15,000 \mathrm{~km} \mathrm{~s}^{-1}$, again using a $\pm 15^{\circ}$ galactic "zone-of-avoidance." The present sample largely includes the LP94 set (as will be qualified further below). For convenience, we will refer to the LP94 set of clusters as the $15 \mathrm{~K}$ sample, while its present augmentation is the $24 \mathrm{~K}$ sample. The $24 \mathrm{~K}$ outer limit of the survey was selected to provide a significantly deeper reference frame than that constructed in LP94, but one that would not be too strongly affected by the limited depth of the Abell and ACO catalogues, which are heavily incomplete beyond $z \sim 0.1$ (Postman et al. 1992).

The present sample is drawn from a considerably larger provisional sample defined by us in the early 1990's based on a literature survey of Abell clusters with measured or 


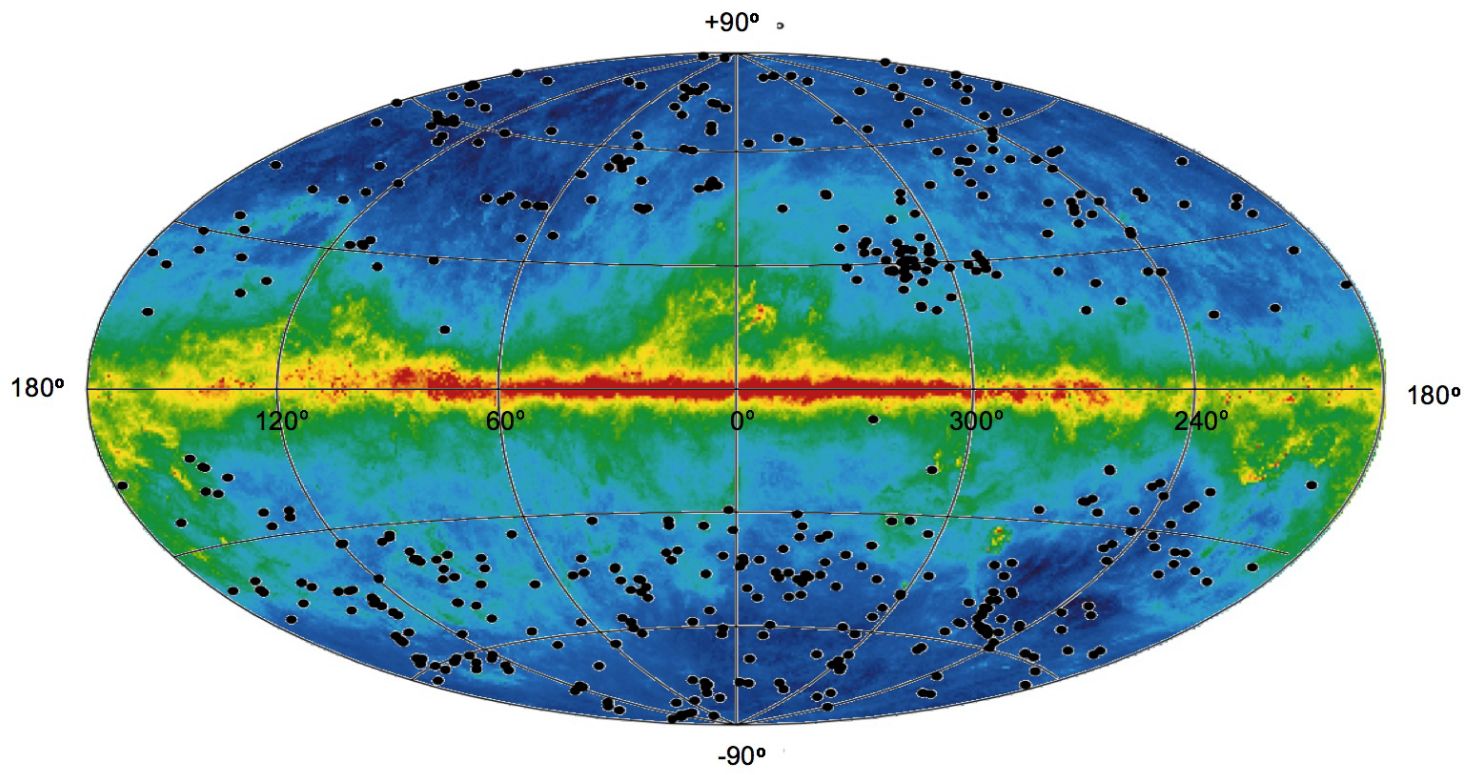

Figure 1. The distribution of the present sample of BCGs is shown in galactic coordinates superimposed over a predicted $94 \mathrm{GHz}$ dust map derived from IRAS and COBE. The dense concentration of clusters at $l \sim 315^{\circ}, b \sim+30^{\circ}$ is due to the combination of the HydraCentaurus and Shapley superclusters. The dust map is a publicly available data product derived using the predict_thermal algorithm by Finkbeiner et al. (1999) and shows the predicted dust emission, in $\mathrm{mK}$ antenna temperature units at $94 \mathrm{GHz}$, using their 2-component model 8.

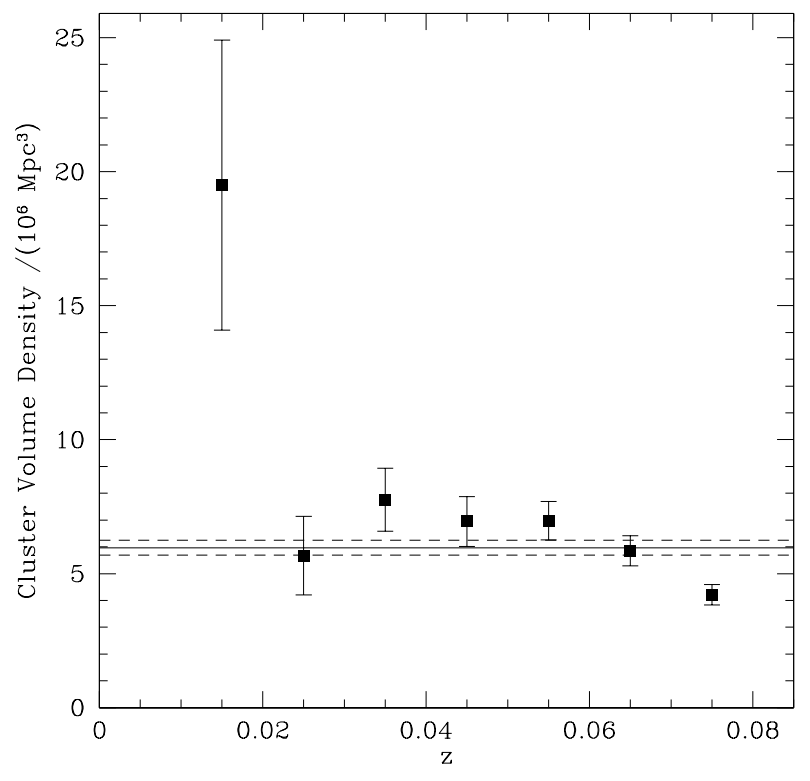

Figure 2. The comoving volume density of the clusters in this study, binned in $\Delta z=0.01$ shells as a function of redshift is shown. The error bars reflect the Poisson errors in the number of clusters in each shell. The horizontal lines give the density (and associated error) of BCGs averaged over the entire volume, but including 53 additional clusters lacking observations or that were excluded for having non-elliptical BCGs. The volume calculation accounts for the $\pm 15^{\circ}$ galactic-plane "zone of avoidance" and the counts in each bin are corrected for the Abell cluster galactic latitude selection function $P(|b|)=\operatorname{dex}(0.3[1-c s c|b|])$.

estimated redshifts. Because we wanted to construct the best sampling of the local volume possible within the limitations of the Abell and ACO catalogues, we were liberal with accepting plausible candidates for the $24 \mathrm{~K}$ sample. As we describe below, the final cluster selection is made with a combination of the latest published redshift surveys and radial velocities that we measured ourselves.

The final set of 433 clusters are those of the larger candidate set for which we observed the BCG, and had sufficient redshift information to determine that the cluster was within the $24 \mathrm{~K}$ redshift limit. There are 38 additional clusters that had well-determined redshifts that placed them within the $24 \mathrm{~K}$ volume, but for which we were unable to image the BCG or sufficient candidates to be confident of the BCG selection. There are 15 more clusters with non-elliptical BCGs, which were also excluded from the sample. These clusters are listed in Table 2. These two sets include 5 clusters observed as part of the $15 \mathrm{~K}$ sample, but that are now deleted from the present sample for a variety of reasons. With the present richer data set we now find that for two of the $15 \mathrm{~K}$ clusters we in fact observed a small foreground group in front of a rich cluster, selected the M2 rather than the correct $\mathrm{BCG}$ in one cluster, and that the BCG is non-elliptical in the remaining two clusters.

The cluster space density as a function of redshift is shown in Figure 2. The cluster counts in each bin have been weighted by the established Abell cluster galactic latitude selection function: $P(|b|)=\operatorname{dex}(0.3[1-c s c|b|])$ (Bahcall \& Soneira 1983). The volume computation accounts for the $\pm 15^{\circ}$ galactic-plane zone-of-avoidance. The average cluster comoving space density over the range $z \leq 0.08$ is $(5.97 \pm 0.28) \times 10^{-6} \mathrm{Mpc}^{-3}$, including the $5 \overline{3}$ missed and non-elliptical BCG clusters. The space density of Abell clusters used in this study is relatively constant, with the exception of the last bin where a significant decline is seen. The positive deviation in the first bin, centered at $z=0.015$, is not statistically significant $(\sim 2.5 \sigma)$.

The completeness of the Abell catalog as a function of richness, redshift, and galactic latitude has been extensively studied. The trends are such that the com- 


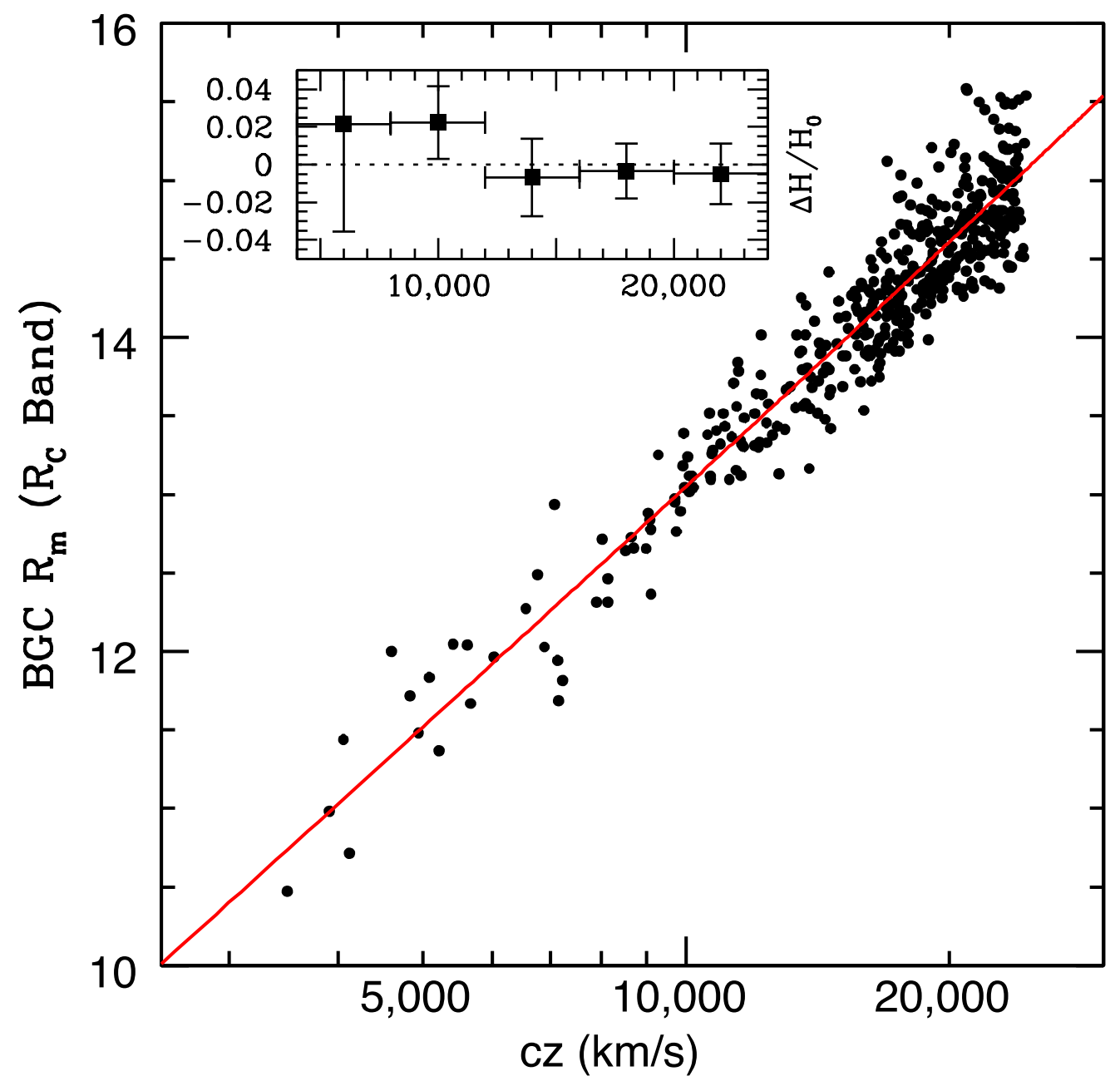

Figure 3. The Hubble diagram derived from the present $24 \mathrm{~K}$ sample, showing BCG apparent metric luminosity as a function of cluster redshift (CMB frame). The red line is the mean theoretical Hubble relationship assuming $\Omega_{m}=0.3$, and $\Lambda=0.7$. The inset shows the binned residuals about relation in shells of $4000 \mathrm{~km} \mathrm{~s}^{-1}$ starting at $4000 \mathrm{~km} \mathrm{~s}^{-1}$, expressed as $\Delta H / H_{0}$. The largest deviation in a shell is $\Delta H / H_{0}=-0.022$, but none is significantly different from zero.

pleteness is lower at lower richness, higher redshift, and lower galactic latitude. More specifically, Postman et al. (2002) show that the detection efficiency as a function of richness class in the Abell catalog is $\sim 55 \%$ for $\mathrm{RC}=0$, $\sim 75 \%$ for $\mathrm{RC}=1$ and is essentially $100 \%$ for $\mathrm{RC} \geq 2$. The richness class distribution in the current $24 \mathrm{~K}$ sample is $55 \% \mathrm{RC}=0,35 \% \mathrm{RC}=1$, and $10 \% \mathrm{RC} \geq 2$. Our study here is immune to the known completeness trends so long as the properties of the BCG in the detected Abell clusters are representative of those in the clusters that were missed during the construction of the northern and southern Abell catalogs.

\subsubsection{A BCG Hubble Diagram}

As a further illustration of the sample geometry and its utility as a probe of the Hubble flow within the local volume, we show a Hubble diagram derived from our BCG sample in Figure 3. The details of the sample selection, reduction, and analysis of the photometry needed to generate this figure are the subject of much of the rest of this paper. For now, the relevant details are that the velocities are mean cluster velocities in the cosmic microwave background (CMB) frame, and the photometry is the apparent metric luminosity, $R_{m}$, of the BCGs, but with extinction and k-corrections applied. The photometry has also been corrected to $\alpha=0.5$ using the relationship (equation 8) between metric luminosity and $\log \alpha$, a parameter measuring the slope of the photometric curve-of-growth at the metric radius, $r_{m}$.

The Hubble diagram shows that the number of galaxies per velocity interval, rises with distance as $\sim D^{2}$, as expected for a survey with a roughly constant cluster density with redshift. The sharp cut-off at the $24 \mathrm{~K}$ velocity limit is also evident. The rms scatter about the nominal theoretical relation specified by $\Omega_{m}=0.3$, and $\Lambda=0.7$ is $0.271 \mathrm{mag}$. We can constrain any departures from the expected Hubble flow as a function of redshift by binning the residuals about the Hubble relation. Since our sample is full sky, this effectively tests for monopole variations in the Hubble flow with 
distance. Figure 3 shows the mean residuals in shells of $4000 \mathrm{~km} \mathrm{~s}^{-1}$ starting at $4000 \mathrm{~km} \mathrm{~s}^{-1}$, expressed as $\Delta H / H_{0}$. All but the innermost shell are consistent with $\Delta H / H_{0}=0$ at the $<2.0 \%$ level; the innermost shell is also consistent with $\Delta H / H_{0}=0$, but with a poorer $5.7 \%$ error due to the small number of BCGs interior to 8000 $\mathrm{km} \mathrm{s}^{-1}$. The present result is completely consistent with the BCG Hubble diagram derived from the earlier $15 \mathrm{~K}$ sample (Lauer \& Postman 1992), but with errors nearly a factor of two smaller.

\subsection{Selection of the BCGs}

We define the BCG to be the brightest member (in the $R_{C}$-band) of the cluster within a $14.3 \mathrm{kpc}$ radius ${ }^{1}$ "metric aperture" centered on the galaxy (and with close or embedded companions photometrically subtracted), with the proviso that the galaxy must also be an elliptical. The use of metric BCG luminosity as a distance indicator was initially advanced by Gunn \& Oke (1975), and developed further by Hoessel (1980) and PL95. As demonstrated in PL95, our particular choice of the metric aperture minimizes the scatter in the average BCG luminosity. The aperture is large enough to include a large fraction of the total luminosity of the BCG, but avoids the difficulty of measuring a total magnitude for the BCG, which requires surface photometry at very faint levels and large angular radii in a rich-cluster environment. This problem has limited the accuracy of a number of recent studies of BCGs. The photometry provided by the SDSS and 2MASS surveys, for example, strongly underestimates the total luminosity of low- $z$ BCGs (Lauer et al. 2007). The SDSS photometry suffers from oversubtraction of the sky background, while the 2MASS total magnitudes are based on a profile model that fails to include the extensive envelopes of the galaxies.

As with the definition of the cluster sample, we were liberal with observing all plausible BCG candidates for any given cluster, making the final choice only when all the observations were in hand. The initial selection of BCG candidates was done visually from digitized skysurvey plates, augmented with velocity information when available. Unless one galaxy was strongly dominant and known to be in the cluster from its redshift, we would typically select several bright elliptical galaxies for imaging and spectroscopic observations, with the final selection based on CCD aperture photometry and knowledge of the cluster redshift.

As noted in PL95, the BCGs in the $15 \mathrm{~K}$ sample were often displaced in angle and/or velocity from the nominal cluster center, thus we attempted to select all bright elliptical galaxies within the nominal Abell radius of the cluster, rather than the brightest "central" galaxy. The Abell radius is $1.5 h^{-1} \mathrm{Mpc}$ or $2.1 \mathrm{Mpc}$ for the present cosmological parameters. ${ }^{2}$ One of the questions that will be considered in the later sections is the extent to which the BCG is in fact displaced from the cluster spatial and velocity centroids - allowing for the possibility that the

1 This is the same as the $10 h^{-1} \mathrm{kpc}$ radius used in LP94 and PL95.

2 This is somewhat larger than the over-density radius, $r_{200}$, (Carlberg et al. 1997) which has a median value of $1.7 \mathrm{Mpc}$ for the present sample, and is often used as a proxy for the physical extent of a galaxy cluster.
BCG may be significantly offset from either is critical to the BCG selection.

The selection of the BCG can be complex, and different surveys may disagree on which galaxy is the BCG in any given cluster. As one example, we compared our selection to those from von der Linden et al. (2007), who extracted their sample from the SDSS-based C4 cluster catalogue (Miller et al. 2005), using isophotal magnitudes for BCG luminosity. Of the $429 \mathrm{C} 4$ clusters selected by von der Linden et al. (2007) that should be within our redshift limit, only 44 clusters are in our sample. 3 Of the 44 cluster matches, we agreed on the BCG in 33 or $75 \%$ of the clusters. In 8 of the 11 clusters remaining von der Linden et al. (2007) selected a galaxy that we classified as M2, the second-ranked galaxy, based on our photometry. As noted in the next section, this choice may depend on the size of the metric aperture, but we concluded that our M2 would be the BCG based on total flux (see below) in only 3 of the 8 cases. Lastly, for one cluster, A1142, the C4 catalogue identified two clusters, with the BCG for one corresponding to our M2 for A1142.

\subsubsection{A Subset of Bright M2 Members}

As a natural consequence of imaging all plausible BCG candidates, we also imaged a large sample of secondranked cluster members, M2, as based on their metric luminosities. This set is presented in Table 3 . We observed 179 M2 galaxies, corresponding to $\sim 41 \%$ coverage over the total sample of 433 clusters. Of course, we were most likely to observe M2 when it was a close rival to the BCG. We thus have constructed a sample of M2s that are likely to have properties similar to the BCGs. Indeed, many of the M2 galaxies in clusters with more luminous BCGs in fact are more luminous than a significant fraction of the BCGs in other clusters. The M2 sample appears to be nearly complete for galaxies within $0.3 \mathrm{mag}$ of the BCG luminosity in any cluster. Because we were not complete in observing M2s that were not close rivals of the $\mathrm{BCG}$, however, this sample must be used carefully.

We emphasize that because the BCG/M2 selection is based on the metric, rather than total luminosity, there are $14 \mathrm{M} 2 \mathrm{~s}$ (identified in Table 3 that would have been selected as the BCG had a larger aperture been used. These galaxies have $\alpha$ substantially larger than that of their corresponding BCG, such that the integrated flux out to a given radius ultimately exceeds that of the $\mathrm{BCG}$ when the radius is large enough. Because this ambiguity affects only a small portion of the sample, and the radial limits of the surface photometry are heterogeneous at radii well outside the metric aperture, we prefer to preserve the purely metric-aperture based BCG selection. ${ }^{4}$

\subsection{Imaging Observations and Photometry}

\subsubsection{Observations}

\footnotetext{
3 There were 8 additional clusters that might have been in the common set. We were not able to obtain BCG photometry in 4 of them, and the BCGs in the remaining 4 were not elliptical galaxies.

4 All of the M2s flagged as ultimately exceeding the BCG in luminosity do so at only very large radii. In one case, however, for the BCG/M2 pair in A3531, the transition occurred just outside the metric radius, so we designated the initial M2 galaxy as the true BCG.
} 
Images of the BCGs were obtained in 13 runs between 1989 and 1995 using CCD cameras on the KPNO-4m, KPNO-2.1m, and CTIO-1.5m telescopes. The runs are listed in Table 4. The first set of runs from 1989 to 1991 were used for the observations of the $15 \mathrm{~K}$ sample presented in PL95, but are repeated here for convenience. As compared to the first set of runs, the cameras used in the later runs generally had larger fields, allowing for more straight forward estimation of the sky level, as well as improved efficiency for observing multiple BCG candidates in a single observation.

For the PL95 observations of the $15 \mathrm{~K}$ sample, we obtained images in both the Kron-Cousins $R_{C}$ and Johnson $B$ filters. The $R_{C}$-band imagery served as the primary material used for the photometry, with the $B$-band providing auxiliary information to test the validity of the extinction and k-corrections, as well as to test the BCG $B-R_{C}$ color as diagnostic of the properties of the galaxies. In PL95, however, we found that the BCGs had a very narrow range in color $\left(\left\langle B-R_{C}\right\rangle=1.51 ; \sigma_{\left(B-R_{C}\right)}=\right.$ $0.06 \mathrm{mag})$ that showed no correlation with other properties of the BCGs or with residuals in their photometric distance estimates. We thus elected to only obtain $R_{C^{-}}$ band images for the present sample, given the demands of observing a large number of galaxies in the limits of the observing time available. ${ }^{5}$

To allow for the use of BCGs as photometric distance indicators, we could only obtain useful images under photometric conditions. About $20 \%$ of each night was dedicated to observing Landolt (1983) standard stars. Frequent observation of standards not only allowed the photometric quality of the night to be monitored, but also allowed for frequent characterization of the airmassextinction term, which often varied from night to night, or even over the duration of a single night. Given the very narrow range of color seen in BCGs, we were less concerned with determining the color terms of the cameras, and selected standard stars that closely matched the typical $B-R_{C}$ colors of the BCGs. The median scatter in the standard star photometry over all nights was only $0.008 \mathrm{mag}$, with the two poorest nights having residuals of 0.022 and 0.035 mags.

In addition to obtaining accurate photometric calibration, we also were concerned with accurate flat-field calibration of the images, such that accurate sky levels could be measured. This was done by observing a number of "blank sky" fields during the night to correct for largescale illumination patterns that were not removed by the standard use of dome flat-field images. We could not use the alternative of generating a sky-flat from the stack of images obtained on any given night, since the BCGs are extended and were typically centered in the CCD fields. This procedure reduced the error in the sky levels from several percent to a few tenths of a percent. As we discuss below, the final total error in the metric magnitudes as measured by cross validation is only $0.01 \mathrm{mag}$, demonstrating that any errors associated with the sky subtraction must be less than those contributed by the photometric solution.

${ }^{5}$ 2MASS K-band photometry is too shallow to provide reliable measurements over the metric aperture. See Appendix B in Lauer et al. (2007) for full details.

\subsubsection{Image Reduction and Surface Photometry}

Reduction of the CCD images obtained in the newer set of runs followed the same procedures as were described in PL95. Sky levels were determined from the intensity modes measured in the corners of the images. Surface photometry of the BCG candidates was obtained using the least-squares isophote-fitting algorithm of Lauer (1986). In brief, the algorithm describes the galaxies as a nested set of concentric elliptical isophotes, which are allowed to have arbitrary surface brightness, ellipticity, and position angle as a function of radius. The key feature of the algorithm is that it allows galaxies in the images to overlap; indeed it was developed explicitly to decompose "multiple-nucleus" BCG into individual galaxies. In multiple systems, overlapping, merging, or even luminous galaxies completely embedded in the BCG are modeled and subtracted from the envelope of the $\mathrm{BCG}$ prior to measurement of the metric luminosity. Again, no assumed form of the surface brightness profile was imposed. The algorithm also allows bad pixels, bright stars, compact galaxies, dust patches, and so on, to be excluded from the surface photometry solution.

Once the surface photometry for all the galaxies in an image was completed, model images were reconstructed from the surface brightness profiles and their total flux integrated in a geometric series of circular apertures centered on the galaxy. This is the final form of the photometry used for the subsequent analysis. The actual value of the luminosity with the metric aperture is obtained by using cubic splines to interpolate among the series of apertures, based on the final velocity adopted for any given cluster. This representation is highly accurate; the $1-\sigma$ difference between the surface photometry integrated over the metric aperture versus integrating over the galaxy image directly (carefully cleaned of contaminating sources) is only $0.003 \mathrm{mag}$.

Lastly, the photometry is corrected for galactic extinction and the filter K-correction, as was done in PL95; for the $R_{C}$-band, $K_{R}=\log _{10}(1+0.96 z)$, and $A_{R}=0.59 A_{B}$. Extinction values for the present work are provided by Schlegel et al. (1998), in contrast with PL95, where extinctions from Burstein \& Heiles (1982) were used. Table 1 gives the $A_{B}$ values used.

\subsubsection{Cross Validation of the Photometry}

To provide additional validation of the galaxy photometry, observations of several galaxies were repeated in multiple runs, as was also done in PL95. We also reimaged most of the $15 \mathrm{~K}$ BCGs in the course of obtaining the $24 \mathrm{~K}$ sample. This not only allows the accuracy of any given aperture measurement to be confirmed, but also provides a test for any systematic differences in the photometric zeropoints between the various runs. Because we were concerned with obtaining consistent photometry over the full sky, care was taken to ensure that extensive cross-validation observations between the north/south hemispheres and spring/fall observing seasons were obtained. Figure 4 shows the measured differences in the metric magnitude for galaxies observed across different runs. The data allowed 48 separate inter-run comparisons to be performed, assembled from 260 overlap galaxy observations obtained over the course of the imaging part of the survey. This provided $62 \%$ of the 78 potentially 


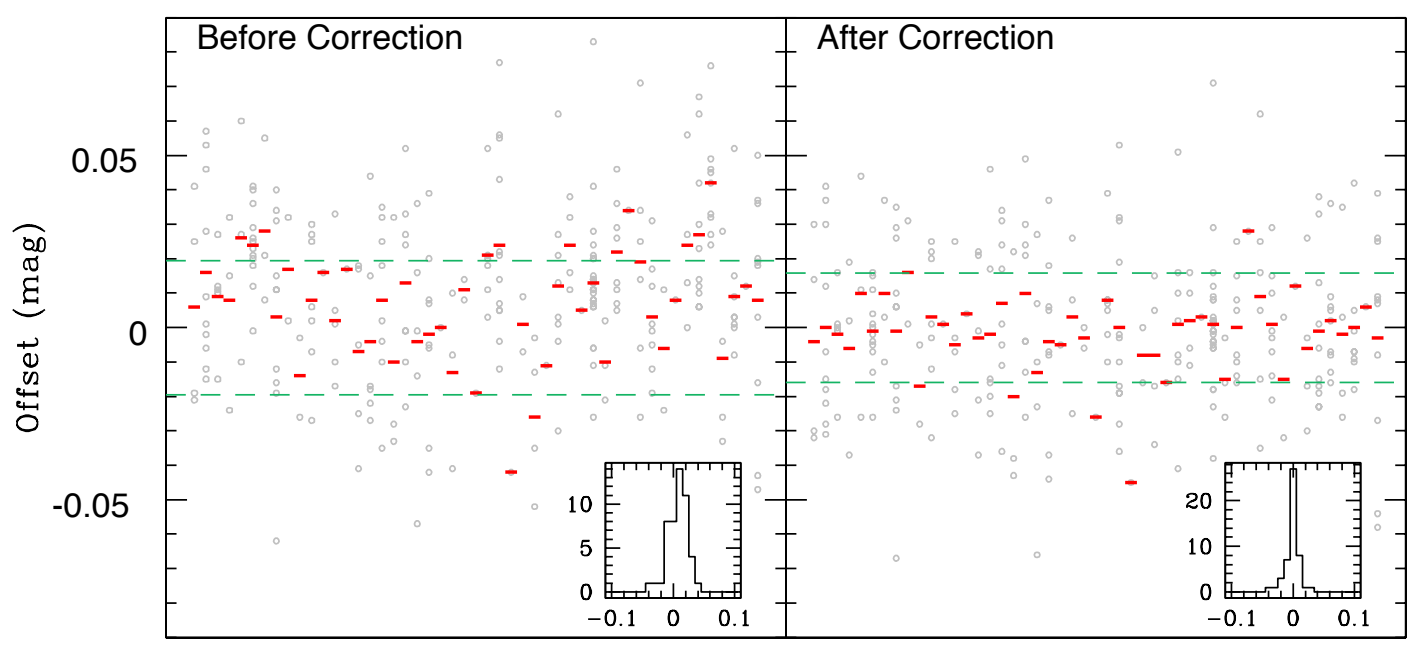

Run Comparison

Run Comparison

Figure 4. The differences between the single observations of the metric magnitude for galaxies observed in common between different observing runs. A total of 48 inter-run comparisons, derived from 260 overlap galaxy observations, are shown. The light grey data points show the individual differences. Red lines show the mean offset for each inter-run comparison. The dashed green lines show the total rms value. The plot on the left shows the results before the global offset corrections are applied to the photometry. The plot on the right shows the residual differences after the offset corrections are applied to each run. The distribution of the average metric magnitude offsets for all 48 inter-run comparisons is shown in the histograms in the lower right of each plot. As the analysis is based on differences between pairs of observations, the photometric error in any single observation will be $\sqrt{2} \times$ smaller on average. After correction, the final total photometric error for any metric magnitude is 0.011 mag.

unique comparisons among the 13 runs, thus densely populating the run/run cross-correlation matrix.

The total rms for all differences between pairs of duplicate galaxy metric magnitudes is $0.0195 \mathrm{mag}$, which implies that the average error in any single measure is $0.0138 \mathrm{mag}$, a factor of $\sqrt{2}$ smaller. By fitting for an average photometric offset correction for each run (done as a simultaneous least-squares fit to the entire ensemble of overlap observations), we can reduce the total difference rms to $0.0159 \mathrm{mag}$, or $0.0112 \mathrm{mag}$ for any single observation. Because the average photometric correction for any single run is small, $0.0104 \mathrm{mag}$, we have chosen not to apply the corrections in the present work; however, systematic differences between runs are more important for the measurement of large scale deviations from the Hubble flow, and will be considered in our use of the present sample as a velocity reference frame.

\subsection{Spectroscopic Observations}

We obtained long-slit spectra of all BCG candidates in the sample over the course of 14 observing runs, spanning a five year timeframe, at NOAO's Cerro Tololo InterAmerican Observatory (CTIO) and Kitt Peak National Observatory (KPNO). The CTIO observations were done primarily using the Blanco 4-m telescope, except for the first two runs, which used the 1.5-m telescope. All KPNO runs were done using the Goldcam spectrograph on the $2.1 \mathrm{~m}$ telescope. Table 5 summarizes the instrumental parameters. The slit width was set to 2 arc-seconds. For most observations, two or three independent exposures were obtained (and coadded for further analysis), although in some cases only a single exposure was acquired. The exposure times for each individual exposure varied depending on telescope aperture and the es- timated target redshift. As the overall objective was to use the spectra to obtain both a measurement of the redshift and the internal stellar velocity dispersion, we set integrations to achieve a minimum signal-to-noise ratio of 20 per pixel in the final co-added $1 \mathrm{D}$ spectrum. A total of 842 co-added spectra were obtained for 689 unique galaxies.

Over the course of the survey, we repeatedly observed 13 bright nearby galaxies as radial velocity reference standards. These observations were designed to provide a cross-check on our redshift measurement accuracy over the duration of the program. The mean absolute value of the velocity difference between the reference galaxy redshifts from different runs was $32 \mathrm{~km} \mathrm{~s}^{-1}$ $(\langle\Delta \mathrm{v} / \mathrm{v}\rangle=0.005)$ with an rms scatter of $38 \mathrm{~km} \mathrm{~s}^{-1}$. We also observed a subset of BCGs multiple times, both from this survey and from the earlier LP94 survey to serve as cross-checks between different observing runs and telescopes. For the velocity dispersion estimates, a series of spectra were repeatedly obtained of $27 \mathrm{~K}$-giant stars.

The 2D spectra were corrected for basic instrumental signatures. Bad columns were identified and interpolated over. Bias subtraction was done by first using the overscan region to determine the mean DC level, which was subtracted from the full frame. Bias structure removal was then performed using a series of zero-duration exposures acquired before the start of each night. Quartz lamp exposures were co-added and normalized to provide a flat-field correction frame. Any cosmic ray hits that extended for more than 2 pixels were manually identified and interpolated over where possible. Smaller cosmic ray hits were dealt with during co-addition of the extracted $1 \mathrm{D}$ spectra.

The 1D spectra were extracted and wavelength- 
calibrated using IRAF's NOAO onedspec package. The extraction was done using a 3rd order Legendre polynomial function to allow the aperture center to track any significant spectral curvature along the dispersion axis. The average spectrum extraction aperture width was 9 arcseconds (rms 2.5 arcsec), which is significantly larger than the typical FWHM seeing $(\sim 1.5$ arcsec $)$ for any given observation. The 9 arcsec width corresponds, on average, to a projected physical width of $10 \mathrm{kpc}$ ( rms $3.6 \mathrm{kpc}$ ). The background level was estimated in two 15pixel wide regions on either side of the source spectrum with a 15 pixel gap between the center of the source spectrum and the start of the background sampling regions. Two iterations of $3 \sigma$ rejection were done during both spectrum tracing and background level determination to reduce susceptibility to cosmic rays. Occasionally, spectra for other galaxies (in addition to the BCG) fell along the slit. We extracted these spectra as well in hopes of providing additional redshift information for the clusters.

The extracted 1D spectra were wavelength-calibrated by extracting identical regions of the companion arc lamp spectra obtained either just before or just after each galaxy spectrum. Helium-Neon-Argon arc lamps were used for these observations. The IRAF dispcor routine was used to perform the wavelength calibration. We typically used a 3rd order polynomial wavelength solution. The wavelength calibration was checked both by looking at the fit residuals provided in the IRAF identify and reidentify routines, and by confirming that the prominent night sky emission lines appeared at their proper central wavelengths. A final co-added 1D spectrum for each object observed on a given night was then produced from the individual wavelength-calibrated 1D spectra using the IRAF scombine routine. Any cosmic ray artifacts that may have survived the co-addition were manually removed via interpolation using IRAF's splot routine.

\subsubsection{Redshift Measurements}

Redshifts were measured using the IRAF-based RVSAO package xcsao. We used eight independent high $\mathrm{S} / \mathrm{N}$ spectral templates of elliptical galaxies to perform the cross-correlations. These 8 templates include spectra of M32, NGC3379, NGC4648, NGC7331, the BCG in Abell 779, and three different composite spectra of low redshift early type galaxies. Eight templates are chosen to allow an estimate to be made of any systematic errors in the cross-correlation measurement. Regions around prominent night sky lines $(\mathrm{Hg}, \mathrm{NaD}, \mathrm{OI})$ and strong atmospheric $\mathrm{OH}$ absorption bands were excluded from the fitting procedure. A galaxy redshift for each object was computed by first rejecting the templates with the highest and lowest redshift value and then averaging the results for the remaining six templates. For nearly all our high $\mathrm{S} / \mathrm{N}$ spectra, however, all eight templates yielded consistent redshift values. The typical dispersion between templates was $30 \mathrm{~km} \mathrm{~s}^{-1}$ and the mean velocity error in our redshifts is $45 \mathrm{~km} \mathrm{~s}^{-1}$. The average Tonry \& Davis (1979) cross-correlation R-value, which quantifies the significance of the peak in the normalized cross-correlation function between the galaxy and template spectra is 8.6, with values ranging from 6 to 15 for the BCG candidates.

About $5 \%$ of our spectra have emission lines (only 8 of these emission line systems are BCGs). We used the IRAF routine rvidlines to measure the redshifts of these objects, We typically were able to identify between 8 to 10 emission features in each spectrum in which emission was present. The velocity error in a typical emissionline based redshift is $30 \mathrm{~km} \mathrm{~s}^{-1}$. Table 6 lists the IDs, celestial coordinates, heliocentric redshifts and errors for galaxies, as well as the mean Tonry \& Davis (1979) $R$ values.

The mean absolute-value velocity difference for $\sim 200$ objects with multiple observations is $39 \mathrm{~km} \mathrm{~s}^{-1}$ with a standard deviation of $41 \mathrm{~km} \mathrm{~s}^{-1}$. The mean absolutevalue velocity difference between our redshift measurements and that from SDSS DR7 (Abazajian et al. 2009) for 82 galaxies in common between the two surveys is 33 $\mathrm{km} \mathrm{s}^{-1}$ with a standard deviation of $31 \mathrm{~km} \mathrm{~s}^{-1}$. In both comparisons, any potential systematic shifts are comparable to or less than the scatter in the common measurements and are also comparable to or less than the individual measurement errors.

\subsubsection{Velocity Dispersion Measurements}

We measured central stellar velocity dispersions from the extracted one-dimensional spectra using a "direct" penalized pixel-fitting method, as implemented the IDL code pPXF ${ }^{6}$ (Cappellari \& Emsellem 2004). We use the pPXF code in combination with single-burst stellar population synthesis models (Vazdekis et al. 2010) based on the empirical MILES stellar library (Sánchez-Blázquez et al. 2006), with a Chabrier-style initial mass function (model "Mbi1.30") ${ }^{7}$. The templates span a range of metallicities $(-1.71<[\mathrm{Z} / \mathrm{H}]<+0.22)$ and single-burst ages $(1 \mathrm{Gyr}<$ age $<17.8 \mathrm{Gyr})$. As part of the fitting process, pPXF finds the linear combination of templates that best reproduces the galaxy spectrum. These models are convolved with the instrumental resolution for each observing run, which is modeled using a low-order polynomial fit to the width of the arclines, and typically varies as a function of wavelength. We allow pPXF to fit for four velocity moments $(V, \sigma, \mathrm{h} 3$, and h4) and use a fourth order multiplicative polynomial to account for continuum mismatch due to imperfect spectral fluxcalibration. We mask regions covering possible strong emission lines (the Balmer lines and [OIII] $\lambda 4959,5007$ ), and run the fit iteratively; on the first run, we identify $\pm 4 \sigma$ outliers in the fit residuals as noise spikes, mask them out, then rerun the velocity fits.

We further experimented with a number of model parameter choices that might introduce systematic effects into the $\sigma$ measurements. The massive BCGs in our sample have non-Solar abundance ratios (in particular, they are enhanced in $\mathrm{Mg}, \mathrm{CN}$, and $\mathrm{C}_{2}$, e.g., Graves et al. 2007; Greene et al. 2013), while the stellar population templates have Solar-scale abundance patterns. We experimented with masking the absorption line regions strongly affected by these non-Solar abundances, but this had a negligible impact on the resulting $\sigma$ measurements. We also investigated the effects of using the pPXF "BIAS" keyword to push the velocity solution toward low values of $\mathrm{h} 3$ and $\mathrm{h} 4$. This also had a negligible effect on our derived $\sigma$ values. Fitting for only two velocity moments (i.e., setting h3 $=\mathrm{h} 4=0$ ) in some cases produced

\footnotetext{
${ }^{6}$ http://www-astro.physics.ox.ac.uk/ mxc/idl/

7 http://miles.iac.es//pages/ssp-models.php
} 

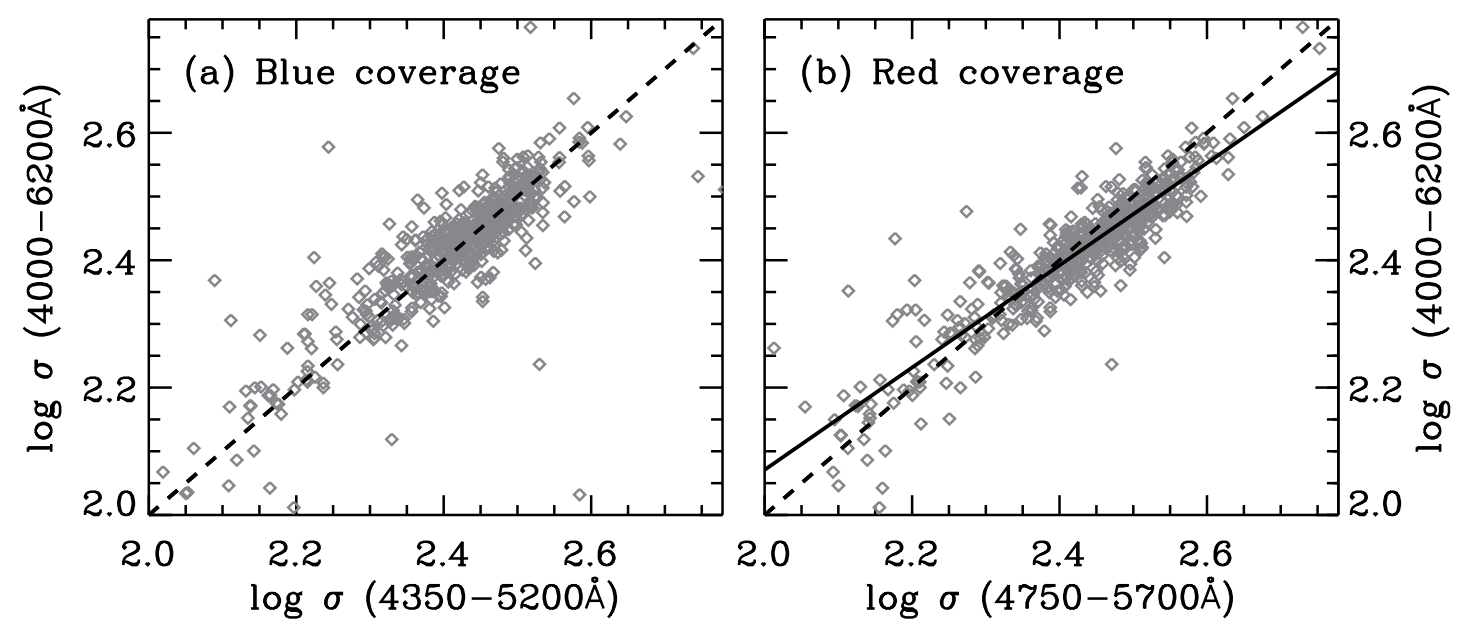

Figure 5. Comparing velocity dispersions measured from the "full" spectral range versus those measured from the more limited "blue" or "red" wavelength coverage. Open diamonds show individual galaxies in our sample. Dashed lines show a one-to-one relation. When only the blue wavelength range is used, the recovered velocity dispersions agree with those derived from the full spectral range (panel a). When only the red wavelength range is used, the measured $\sigma$ values are biased high for high- $\sigma$ galaxies (panel b). We use a linear fit to the correlation to correct $\sigma$ values measured in the red when the full spectral range is not available (solid line).

a modest increase in the derived $\sigma$ values, as did using a higher-degree multiplicative polynomial for the continuum adjustment. There was little effect for galaxies with $\sigma \sim 150 \mathrm{~km} \mathrm{~s}^{-1}$, but increases of $\sim 20 \mathrm{~km} \mathrm{~s}^{-1}$ for galaxies with $\sigma \sim 350 \mathrm{~km} \mathrm{~s}^{-1}$ were seen. We elected to use the fourth order multiplicative polynomial and unbiased four moment velocity fits for our final measurements.

By far the largest systematic effect was the choice of rest-frame wavelength interval used in the velocity fits, with $\sigma$ values biased by up to $\sim 40 \mathrm{~km} \mathrm{~s}^{-1}$ when comparing different wavelength intervals. Our observations were not acquired with uniform wavelength coverage (see Table 5). Accordingly, we define three rest-frame wavelength ranges to use in our analysis. The "full" range of

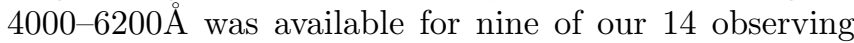
runs. We also defined a more limited "blue" range of 4350-5200A for runs CT92F, CT93S, and KP92S, and a

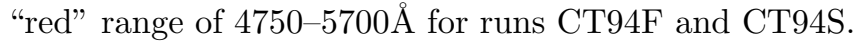
For the nine runs with full wavelength coverage, we measured $\sigma$ from the "blue", "red", and "full" wavelength separately, in order to calibrate the effect of differing wavelength coverage as described below.

Due to imperfect data archiving, we were only able to retrieve pixel-by-pixel error arrays for a subset of the observing runs. The pPXF code uses error spectra both to penalize low-S/N or bad pixels in the fitting process and to estimate errors in the derived parameters, such as $\sigma$. We were able to use the iterative outlier rejection described above to mask bad pixels, with all other pixels being assigned equal weight. In order to estimate the uncertainties in measured values of $\sigma$, we again resorted to iterative use of the pPXF code. In the first run, all pixels were simply assigned equal (and arbitrary) errors. pPXF outputs the residuals between the bestfitting template combination and the observed spectrum, which have Gaussian scatter about zero. We used the width of the scatter as an estimate of the typical flux error per pixel, then reran pPXF using this value as the input error for all pixels to propagate through the resulting uncertainties in $\sigma$. Where the true error spectra were available, we could compare these estimated errors in $\sigma$ with the true errors; the difference in error estimates was Gaussian with a mean offset of $0.63 \mathrm{~km} \mathrm{~s}^{-1}$ and width of $0.75 \mathrm{~km} \mathrm{~s}^{-1}$. This means that where we could compare them, the bootstrapped error estimates agreed with the true statistical error estimates to within $1-2 \mathrm{~km} \mathrm{~s}^{-1}$. This made us confident that bootstrapped errors could be used reliably for observations whose error spectra had been lost. Overall, the typical uncertainty in our $\sigma$ measurements is $\sim 14 \mathrm{~km} \mathrm{~s}^{-1}$, but varies substantially between observations, depending on the spectral $\mathrm{S} / \mathrm{N}$ and wavelength coverage.

For runs with full wavelength coverage, we compared the $\sigma$ measurements from the full $4000-6200 \AA$ range to

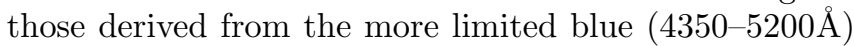

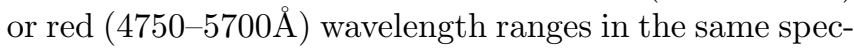
tra, as shown in Figure 5. This calibration demonstrates that when only the blue coverage is available the resulting $\sigma$ measurements are unbiased. The mean offset between the blue and full coverage $\sigma$ values is $-3.2 \mathrm{~km} \mathrm{~s}^{-1}$, with rms scatter of $19.6 \mathrm{~km} \mathrm{~s}^{-1}$ and no clear trend with $\sigma$. In contrast, measurements made with only the red coverage show substantial bias; the mean offset is $+9.3 \mathrm{~km}$ $\mathrm{s}^{-1}$ but increases to $\sim 30 \mathrm{~km} \mathrm{~s}^{-1}$ for the highest $\sigma$ galaxies, with similar scatter of $20.7 \mathrm{~km} \mathrm{~s}^{-1}$. To put all of our targets onto the same effective system, we fit a line that defines the "correction" from the red coverage onto the full coverage values. This correction is applied to the $\sigma$ measurements from the CT94F and CT94S runs, which only have the red wavelength coverage. No correction is applied to the runs with blue coverage.

The spectroscopic observations include many repeat measurements of individual targets, usually in different runs. These can be used to test the internal consistency of our $\sigma$ measurements. Using only $\sigma$ measurements made from the full spectral coverage, we find that differences between repeat measurements of galaxies are Gaussian distributed with a standard deviation of 16.5 $\mathrm{km} \mathrm{s}^{-1}$. This is comparable to the expected typical statistical error of $14 \mathrm{~km} \mathrm{~s}^{-1}$, suggesting that the $\sigma$ measure- 


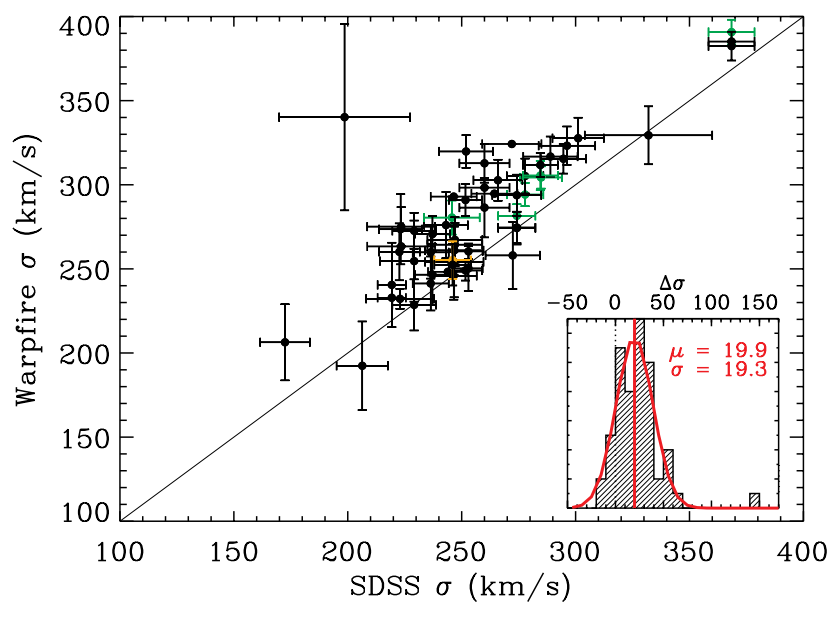

Figure 6. A comparison of our $\sigma$ measurements versus those from the SDSS spectroscopic survey for galaxies in common. Black, green, and orange data points are from the KP96S, KP93S, and KP92F runs, respectively. The solid line shows the one-to-one relation. The inset panel shows a histogram of the offsets between the present and the SDSS measurements. These are Gaussian distributed (red curve) with a mean offset of $19.9 \mathrm{~km} \mathrm{~s}^{-1}$ and scatter of $19.3 \mathrm{~km} \mathrm{~s}^{-1}$. The scatter is comparable to what is expected from the combined present and SDSS observational errors. We do not correct our $\sigma$ measurements onto the SDSS system, but merely note that they are offset to higher values.

ments are stable across the various runs. This is not a trivial statement, given that the observations use two different telescopes, different instruments and instrumental configurations, and span multiple years including instrument upgrades. Comparing repeat observations on a runby-run basis, the runs showing the largest mean offsets from the rest are KP92S (-18.6 $\mathrm{km} \mathrm{s}^{-1}$ for 16 galaxies), CT93S (17.3 $\mathrm{km} \mathrm{s}^{-1}$ for 4 galaxies), and KP94F (-12.3 $\mathrm{km} \mathrm{s}^{-1}$ for 12 galaxies). Notice that none of these deviant runs are the "corrected" runs with only red wavelength coverage. All other runs show offsets that are $<10 \mathrm{~km}$ $\mathrm{s}^{-1}$ from the aggregate.

Where multiple observations are available, rather than averaging the individual measurements, we assign a "best" measurement for each galaxy. For the vast majority of the sources, the various observations agree within the estimated $3 \sigma$ errors; for these sources, the best measurement is the one with the smallest formal error in $\sigma$ (i.e., that measured from the highest-S/N spectrum). For the six galaxies where repeat measurements show catastrophic disagreement $(>3 \sigma)$, we choose the best observation based on the following criteria: full wavelength coverage is preferred over limited red or blue coverage, spectra with noticeable flux calibration or sky subtraction issues are disfavored, higher $\mathrm{S} / \mathrm{N}$ is preferred over low $\mathrm{S} / \mathrm{N}$, and better wavelength coverage is preferred over higher $\mathrm{S} / \mathrm{N}$. These measurements and calibrations result in a sample of $689 \sigma$ "best" measurements among our galaxies.

Finally, 78 of our galaxies are in the SDSS spectroscopic survey, making it possible to compare our $\sigma$ measurements to those from the SDSS spectroscopic pipeline (Bolton et al. 2012, see Adelman-McCarthy et al. 2008 for a comparison of different velocity dispersion algorithms in SDSS). This comparison is shown in Figure 6 . The overlapping galaxy sample is mostly from the KP96S run (black points), with a few from KP93S (green points) and one from KP92F (orange point). The solid line shows the one-to-one relation. The inset panel shows a histogram of the differences between our $\sigma$ and the SDSS $\sigma$ measurements. These have a Gaussian distribution with a mean offset of $19.9 \mathrm{~km} \mathrm{~s}^{-1}$ and width of $19.3 \mathrm{~km} \mathrm{~s}^{-1}$. The scatter compares favorably with the estimated statistical errors in the measurement $(\sim 17$ $\mathrm{km} \mathrm{s}^{-1}$ for the typical errors from our observations and those from the SDSS combined in quadrature) and the internal consistency of our repeat measurements $(16.5 \mathrm{~km}$ $\left.\mathrm{s}^{-1}\right)$. However, there is a significant systematic offset of $\sim 20 \mathrm{~km} \mathrm{~s}^{-1}$. The larger angular apertures $\left(\sim 9^{\prime \prime}\right)$ of the spectral extractions used in this work relative to the $3^{\prime \prime}$ fiber apertures used in SDSS may explain some of the systematic shift if the trend of increasing stellar velocity dispersion with increasing radius, like that seen in the BCG in Abell 383 (Newman et al. 2011), is typical. We do not attempt to "correct" our values onto the SDSS system, but note that work combining $\sigma$ measurements from different sources and spectral reduction pipelines must take such systematic variations into account. Dispersion values for the BCGs and M2 galaxies are listed in Tables 7 and 8 , respectively.

\subsection{Derivation of the Cluster Redshifts}

Cluster redshifts were derived based on galaxy velocities drawn from the database maintained by the NASA Extragalactic Database (NED), augmented by velocities measured for the BCG candidates by us, and SDSS Data Release 7 spectroscopy (Abazajian et al. 2009) where available. The "biweight" estimator of Beers et al. (1990) was used to calculate the mean cluster redshifts, $V_{c}$, and velocity dispersions, $\sigma_{c}$. The initial calculations used galaxies within $\pm 3000 \mathrm{~km} \mathrm{~s}^{-1}$ of the nominal BCG or estimated cluster redshifts, and the cluster Abell radius. While the biweight statistic is designed to be robust in the presence of background or foreground contamination, we still considered it prudent to remove obvious background contamination or other complexities, such as overlap with nearby clusters or groups. This was done by ad hoc inspection of the velocity maps and histograms for each cluster. A second robust statistic introduced by Beers et al. (1990) was used to estimate the cluster velocity dispersion for clusters with four or more velocities.

Table 1 lists the final number, $N_{g}$, of galaxy velocities used to compute the mean velocity and dispersion. This parameter is used as a general marker for the quality of both parameters. For some evaluations of the peculiar velocities of the BCGs within their clusters, we will restrict the analysis to clusters with $N_{g} \geq 50$, to minimize the effects of the error in the mean velocity. For analyses requiring accurate $\sigma_{c}$, we require the clusters to have $N_{g} \geq 25$.

\section{THE LOCATION OF BCGS IN THEIR GALAXY CLUSTERS}

\subsection{The Peculiar Velocities of BCGs}

The stereotypical image of a galaxy cluster has the BCG centrally located, both in projected angular coordinates and radial velocity relative to other cluster members. Studies of clusters with rich enough velocity sampling such that an accurate mean cluster redshift can be estimated, however, show that the BCG may often 


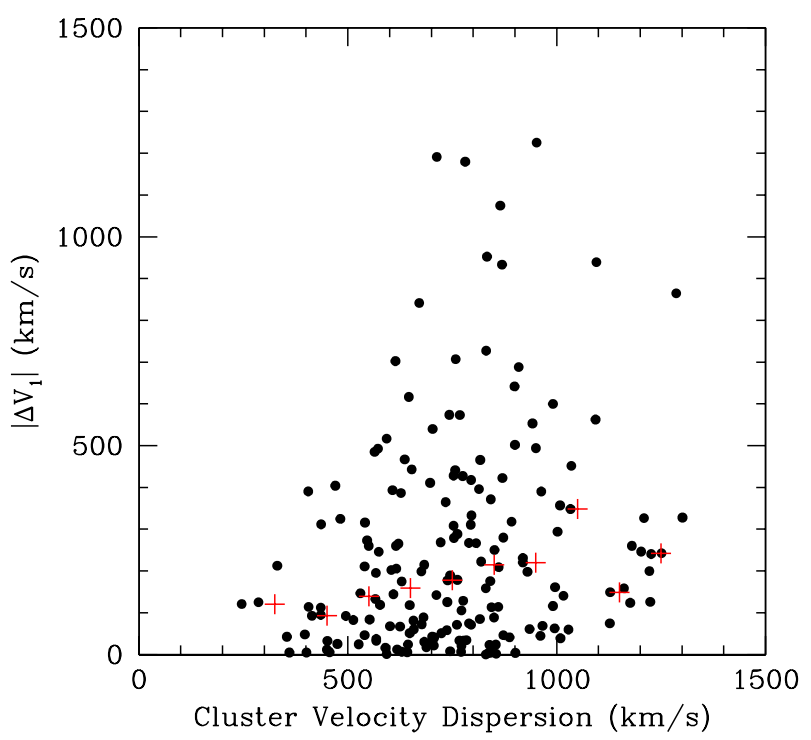

Figure 7. The distribution of $\left|\Delta V_{1}\right|$, the absolute value of the peculiar velocity of the BCG within the cluster as a function of cluster velocity dispersion, for the 178 clusters with 50 or more galaxies with measured redshifts. The red crosses show the median peculiar velocity for each interval of $100 \mathrm{~km} \mathrm{~s}^{-1}$ in $\sigma_{c}$ (except for the first bin, which runs from 275 to $400 \mathrm{~km} \mathrm{~s}^{-1}$ ).

have a significant "peculiar velocity" with respect to their hosting cluster (Zabludoff et al. 1990: Malumuth 1992, Zabludoff et al. 1993; Oegerle \& Hill 2001). PL95 obtained the distribution of BCG $\Delta V_{1} \equiv\left(V_{1}-V_{c}\right) /(1+z)$ for 42 clusters with 20 or more member velocities, finding that the $\Delta V_{1}$ followed a Gaussian distribution with $\sigma_{\Delta V_{1}}=264 \mathrm{~km} \mathrm{~s}^{-1}$, once the error in cluster mean redshift was accounted for. This value is $\sim 0.4$ of the typical 1 -D cluster velocity dispersion, $\sigma_{c}=666 \mathrm{~km} \mathrm{~s}^{-1}$, of the same subset of clusters. To test the hypothesis that the BCG peculiar velocities may be related to their masses, merger histories, and ages, we have derived the BCG peculiar velocity distribution function and investigated the relationship of this parameter to other BCG properties. The distribution function is considered in this section, while the relationship of BCG peculiar velocities to other BCG properties will be considered later in the paper.

Figure 7 shows the absolute values of $\Delta V_{1}$ as function of cluster velocity dispersion for the 178 clusters in the present sample that have 50 or more member velocities. With this level of velocity information the error in the mean cluster velocity is $\sim 100 \mathrm{~km} \mathrm{~s}^{-1}$ or less, allowing relatively small $\Delta V_{1}$ to be detected. As can be seen, most BCGs have $\Delta V_{1}$ well in excess of this error threshold. The median peculiar velocity increases with $\sigma_{c}$. A powerlaw fit shows

$$
\left|\Delta V_{1}\right|=152 \pm 15\left(\frac{\sigma_{c}}{600 \mathrm{~km} \mathrm{~s}^{-1}}\right)^{0.66 \pm 0.26} \mathrm{~km} \mathrm{~s}^{-1}
$$

Coziol et al. (2009) studied the distribution of BCG peculiar velocities in a large sample of Abell clusters and also found $\left|\Delta V_{1}\right|$ to increase with $\sigma_{c}$, although they did not quantify the trend.

Figure 8 shows the binned distribution of $\Delta V_{1}$ normalized by the cluster velocity dispersion. Normalizing by $\sigma_{c}$ largely removes any dependence of the am-

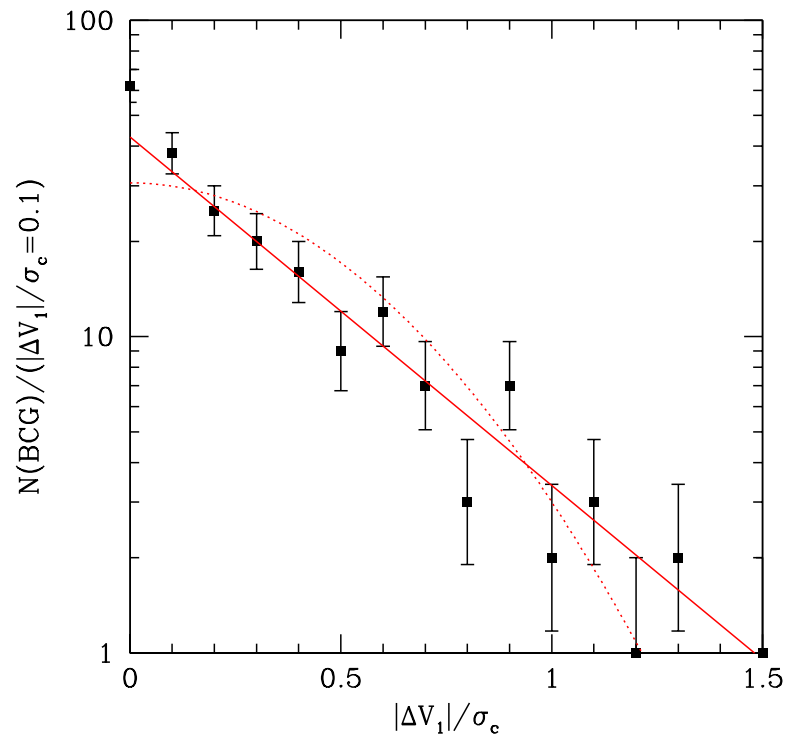

Figure 8. The figure shows the binned distribution of $\left|\Delta V_{1}\right| / \sigma_{c}$, the absolute value of the radial velocity difference between the BCG and mean cluster velocity, normalized by the cluster velocity dispersion, for the 178 clusters with 50 or more galaxies with measured redshifts. The bins are 0.1 units wide. The solid line is an exponential with scale-length 0.39 in $\left|\Delta V_{1}\right| / \sigma_{c}$. The dotted line is the best-fit Gaussian distribution; it is clearly a poorer fit.

plitude of the peculiar velocity on the properties of the cluster itself. Since we are restricting this analysis to clusters with 50 redshifts or more, the errors on $\Delta V_{1} / \sigma_{c}$ will be $<1 / \sqrt{50} \approx 0.14$. In normalized units, the mean $\Delta V_{1} / \sigma_{c}=0.04 \pm 0.04$, with an rms dispersion of 0.49 - note that this number measures a different statistical property of the distribution than does the median peculiar velocity plotted in Figure 7. The two BCGs with the largest $\Delta V_{1} / \sigma_{c}$ values are those in A2399 and A3764, which have $\left|\Delta V_{1}\right|$ of 1191 and $1180 \mathrm{~km} \mathrm{~s}^{-1}$, respectively, or normalized values of 1.67 and 1.51 .

The distribution of $\left|\Delta V_{1}\right| / \sigma_{c}$ is exponential in form. Since the line-of-sight velocity distributions of galaxies in clusters are well known to be Gaussian (Yahil \& Vidal 1977), a random draw of any non-BCG cluster member would, of course, echo this expectation. An exponential velocity distribution specific to the BCGs is thus surprising. The best-fitting exponential distribution is

$$
\ln (N)=-2.54 \pm 0.18\left|\Delta V_{1}\right| / \sigma_{c}+3.76 \pm 0.20,
$$

where $N$ is the number of clusters per bin of width 0.1 in $\left|\Delta V_{1}\right| / \sigma_{c}$. The implied exponential scale length (the reciprocal of the slope given above) is thus $0.39 \pm 0.03$ in $\left|\Delta V_{1}\right| / \sigma_{c}$; this form and scale implies that the median $\left|\Delta V_{1}\right| / \sigma_{c}$ is 0.26 . Note that for small $\left|\Delta V_{1}\right| / \sigma_{c}$, the observed distribution represents the convolution of an unknown intrinsic peculiar-velocity distribution with the error distributions of the BCG and cluster redshifts, plus the errors in the cluster dispersions. However, the observed distribution appears to be smooth and simple in form, thus the intrinsic distribution is likely to transition smoothly from BCGs with $\left|\Delta V_{1}\right| / \sigma_{c} \approx 0$ to those with large $\left|\Delta V_{1}\right| / \sigma_{c}$, where the peculiar velocity of any given $\mathrm{BCG}$ is clearly significant.

The present distribution appears to be similar to that 
measured by Coziol et al. (2009), although they did not characterize it with any functional form. We tested the likelihood that the distribution was non-Gaussian using an Anderson-Darling test (Stephens 1974), finding that a Gaussian distribution is strongly rejected. For the observed rms distribution of $\Delta V_{1} / \sigma_{c}$ of 0.46 , the Gaussian is rejected at the $8 \times 10^{-6}$ significance level. Deletion of the two galaxies that have the largest relative peculiar velocities decreases the rms value of the distribution to 0.44 , and the AD-test allows a Gaussian at $6 \times 10^{-5}$ significance.

It has long been known that the difference in peculiar velocities of pairs of galaxies is exponentially distributed on small scales, both in observations of redshift-space distortions of the two-point correlation function and in simulations (Fisher et al. 1994, Marzke et al. 1995). This effect has been explained in terms of the number, rather than mass, weighting of galaxies in pair statistics (Diaferio \& Geller 1996, Juszkiewicz et al. 1998). We are unaware of an equivalent study of $\mathrm{BCG}$ peculiar velocities in clusters. Reid et al. (2014), in their analysis of redshift-space distortions of SDSS/BOSS galaxies, identified massive halos at $z \approx 0.55$ in a $\Lambda$ CDM N-body simulation containing $2048^{3}$ particles in a box $677.7 h^{-1}$ $\mathrm{Mpc}$ on a side. They measured the difference in peculiar velocity between the most dense spherical region of radius 0.2 times the virial radius, and the cluster overall. Reid (private communication) finds that the distribution of this difference is accurately exponential in a variety of halo mass bins corresponding to rich clusters. While it is unclear whether the Reid et al. identification of the highest-density region in each halo is a good proxy for the BCG, this result is intriguing, and it would be interesting to explore more detailed cluster simulations in which individual subhalos can be identified.

The exponential distribution may reflect a dispersion in the ages of the clusters, the timing of when the BCG was captured by the cluster, or may simply be due to the superposition of Gaussian distributions of different velocity dispersions, weighted by the BCG number distribution (Diaferio \& Geller 1996). BCGs in the tail of the exponential distribution may be those in which the BCG arrived to the cluster in the merger of a group or subcluster relatively recently, and not yet completely relaxed. These ideas could also be explored in simulations, or by looking for correlations between BCG peculiar velocity and signatures of merging in their host clusters.

\subsection{The Projected Spatial Location of BCGs With Respect to the $X$-ray Centers}

The X-ray emission from the intracluster medium provides insight into processes that govern the formation and evolution of the BCGs. Numerous investigations (Edge 1991; Edge \& Stewart 1991; Hudson \& Ebeling 1997:Collins \& Mann 1998; Stott et al. 2012) find significant positive correlations between the total luminosity of the BCG and the X-ray luminosity and X-ray temperature. Schombert (1988) finds that the envelopeluminosity of $\mathrm{cD}$ galaxies, a subset of the BCGs, increases with total cluster X-ray luminosity. Stott et al. (2012) find that the steepness of the $L_{X}-T_{X}$ relation in galaxy clusters correlates with the stellar masses and X-ray offsets of their BCGs. Clusters in which the offset between the BCG position and the peak of the X-ray surface brightness distribution is small tend to be the most regular, most massive systems Allen 1998; Smith et al. 2005; Hudson et al. 2010). Haarsma et al. (2010) find that $\sim 90 \%$ of local $(z<0.2)$ clusters host a BCG within $\sim 30 \mathrm{kpc}$ of the X-ray peak, although their sample is small, and unlikes us, they include a criterion of proximity to the X-ray peak for selecting the BCG from among candidates of "similar" brightness. Brough et al. (2005) find that the structure of the BCG correlates with cluster X-ray luminosity, with the BCG envelope becoming more extended in more luminous clusters.

Our sample is well suited to characterize the precise form of the distribution of the spatial offset, $r_{x}$, of the BCG from the peak of the intra-cluster medium (ICM) $\mathrm{X}$-ray emission, and to assess whether the spatial offset correlates with the velocity offset of the BCG relative to the mean cluster velocity. ${ }^{8}$ In addition, the availability of robust BCG profile shape measurements allows us to determine if the BCG stellar light profile is influenced by the spatial offset.

We cross-correlated our BCG catalog with the ROSAT-based X-ray Brightest Abell-type Cluster Survey (XBACS; Ebeling et al. 1996). XBACS is a fluxlimited catalog derived from the ROSAT All-Sky Survey (RASS; Voges et al. 1999). Of the 283 Abell cluster sources listed in the VizieR version of the XBACS sample, 111 are in common with our current survey. An additional 70 clusters in our current sample have X-ray peak positions from the analysis of the RASS done by Ledlow et al. (2003), which extended to an X-ray flux limit that is $\sim 7$ times lower than that used to derive the XBACS. Chandra X-ray Observatory data were obtained as well for 48 of the ROSAT clusters from the Archive of Chandra Cluster Entropy Profile Tables (ACCEPT; Cavagnolo et al. 2009), which we use in preference to the ROSA' peak positions given the superb Chandra angular resolution. We also searched the literature for XMM data but only 13 of the clusters in our sample have XMM data, too few to provide independent cross-checks on the ROSAT and Chandra samples. We thus focus our X-ray analyses on the above subsample of 174 clusters in our survey, using ROSAT data for 127 clusters and Chandra data for 47 clusters. We note that while Chandra is not a survey mission, the Abell clusters in our survey that have Chandra X-ray temperatures and luminosities that span the same range as the ROSAT temperatures and luminosities. No significant biases are introduced by including the Chandra data in our study of BCG dependence on the X-ray properties of their host clusters.

ROSAT and Chandra have very different on-axis point spread functions: FWHM of $\sim 0.5$ arcseconds for Chandra vs. $\sim 1$ arcminute for ROSAT. We used a sample of 101 Abell clusters (not limited by the redshift limits of our current survey) with observations from both observatories to measure the typical difference, $\theta_{x}$, between the ROSAT peak position and the Chandra peak position. The distribution of $\theta_{x}$ is shown in Figure 9 . The median and mean differences between the ROSAT X-ray position and the Chandra X-ray position are 43 arcsec-

8 Many investigators prefer to normalize $r_{x}$ by a cluster overdensity scale, such as $r_{200}$ or $r_{500}$. Since these latter scales are proportional to $\sigma_{c}$, which only varies by $\sim 2 \times$ over the sample, this would make little difference for the $r_{x}$ distribution, which extends over three orders of magnitude. 


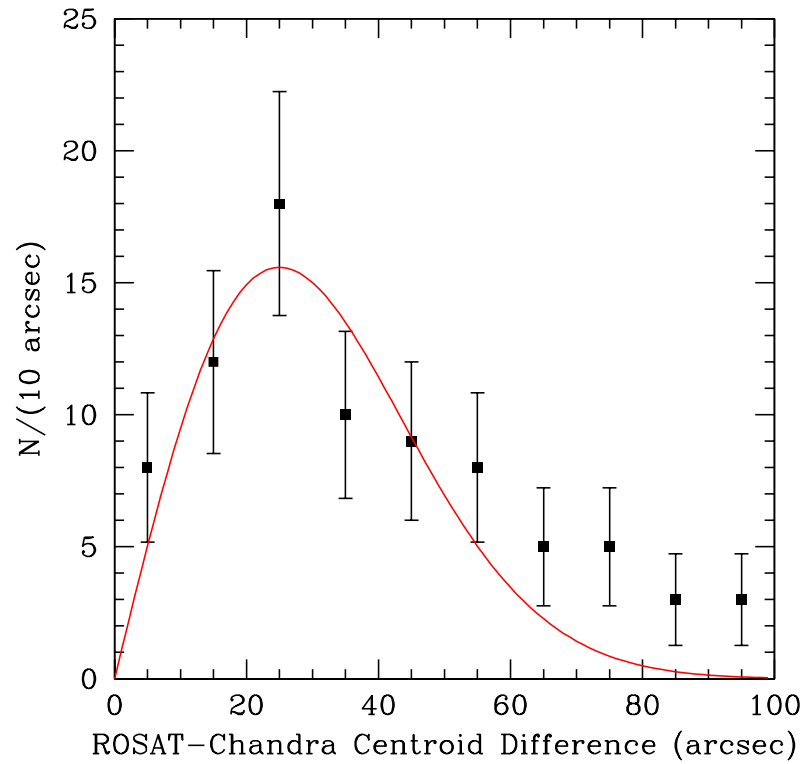

Figure 9. Histograms of the angular differences between Chandra and ROSAT centers for 101 Abell clusters with observations from both observatories. The red line gives a best-fit Rayleigh distribution (equation 3 based on the assumption of a circularly symmetric Gaussian model for the distribution of peak-location differences.

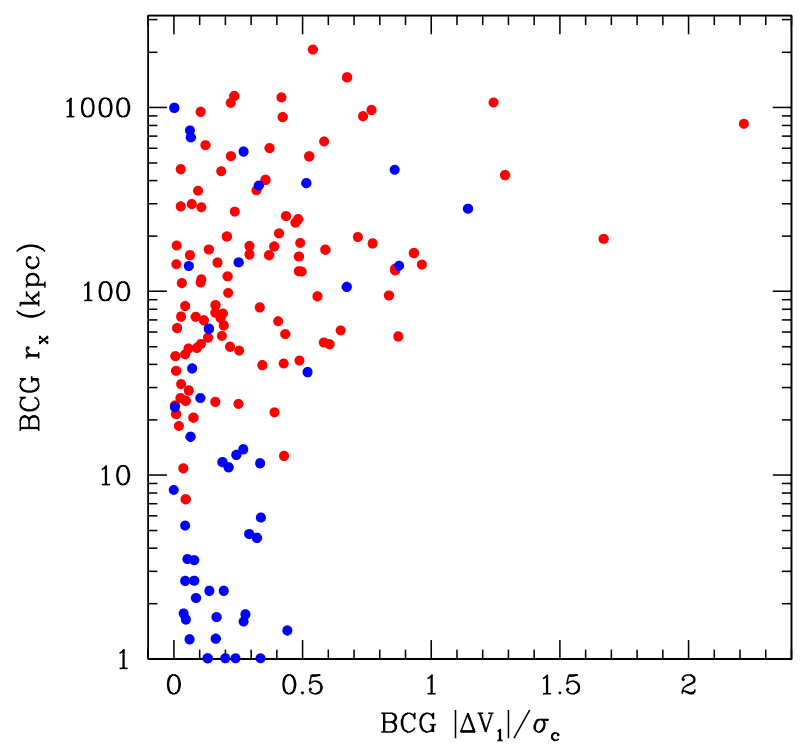

Figure 10. The radial offsets of the BCGs from the cluster X-ray center is plotted as a function of the absolute normalized peculiar velocity of the BCGs within the cluster. Clusters observed with Chandra are blue; those observed with ROSAT are red. Small radial separations $\left(r_{x}<40 \mathrm{kpc}\right)$ always correspond to small peculiar velocities $\left(\left|\Delta V_{1}\right| / \sigma_{c}<0.5\right)$, while large peculiar velocities $\left(\left|\Delta V_{1}\right| / \sigma_{c}>1\right)$ always correspond to large spatial offsets $\left(r_{x}>200\right.$ $\mathrm{kpc})$.

onds and 69 arcseconds, respectively. The median and mean difference between the ROSAT and the Chandra peak X-ray positions in projected physical distance units derived from cluster redshift information are $68 \mathrm{kpc}$ and $121 \mathrm{kpc}$, respectively.

We characterize the distribution of differences between the location of the Chandra and ROSAT X-ray peaks

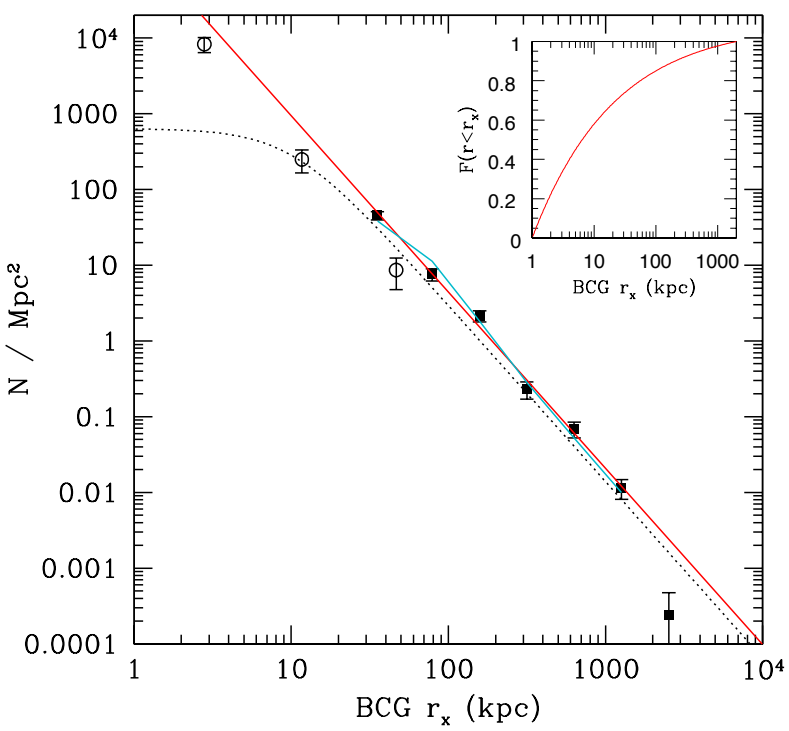

Figure 11. The surface density distribution of BCGs with respect to the cluster X-ray center. The normalization is set to provide a unit integral over the distribution (every cluster has one BCG). Solid points give the density of the full set of BCGs with measured $\mathrm{X}$-ray offsets. The innermost bin extends from the center to 50 kpc; subsequent bins have outer limits geometrically increasing by a factor of two. Open points are the subset of BCGs that have cluster centers provided by Chandra, and thus are not resolutionlimited on this scale. The inner bin from this set extends from the origin to $4 \mathrm{kpc}$, with the next two bins increasing geometrically by a factor of four. The red line is a power-law of index $\gamma=-2.33$ fitted only to the full-set bins. The dotted line is a $\gamma=-2.33$ power-law with a $10 \mathrm{kpc}$ core (equation 5). The blue line is this form as "observed," blurring it with a Gaussian with dispersion $\sigma_{x}$ for the fraction of the clusters observed by ROSAT. The inset gives the cumulative integral of the pure power-law running from $1 \mathrm{kpc}$ to $2 \mathrm{Mpc}$.

on the assumption that the distribution can be modeled as a circularly symmetric Gaussian. This will be almost entirely due to the large ROSAT PSF, but it may be more compact than that, given that the appropriate source of variance is the error in the ROSAT centers, rather than the width of the ROSAT PSF itself. The distribution of the total angular differences between the X-ray peaks will be a Rayleigh distribution,

$$
p\left(\theta_{x}\right)=\sigma_{x}^{-2} \exp \left[\frac{-\theta_{x}^{2}}{2 \sigma_{x}^{2}}\right] \theta_{x} d \theta_{x},
$$

where $\sigma_{x}$ is the dispersion of the Gaussian, as well as the peak location of the above distribution. We fitted this form to $\theta_{x}$ in 10-arcsecond bins, limited to the six bins around the peak of the distribution shown in Figure 9 to avoid the effects of extreme outliers. We measure $\sigma_{x}=25 \pm 3$ arcsec, or an implied FWHM of 60 arcsec for the underlying position-error Gaussian. The adopted distribution given by equation (3) for this $\sigma_{x}$ is also plotted in Figure 9.

Figure 10 shows the offset between the BCG position and ROSAT position as a function of the velocity offset between the BCG and the cluster for the 174 clusters in our sample with ROSAT data. The somewhat triangular shape of the distribution of points shows that there is an overall correlation between the BCG spatial and velocity offsets. In particular, $\left|\Delta V_{1}\right| / \sigma_{c}>1$ occurs only for clus- 
ters with $r_{x}>200 \mathrm{kpc}$, while all $r_{x}<40 \mathrm{kpc}$ clusters have $\left|\Delta V_{1}\right| / \sigma_{c}<0.5$. Stated qualitatively, BCG that are close to the X-ray center in projection always have relatively small velocities. Of course, with a large enough sample, there should be BCGs with high velocities seen in projection against the center; however, $r_{x}<40 \mathrm{kpc}$ corresponds to a very small portion of the projected area of the galaxy clusters.

Figure 11 shows the radial distribution of $r_{x}$ for the subset of BCGs with cluster X-ray centers as a plot of BCG surface density as a function of radius. The normalization is set so that the cumulative integral over the surface density for each cluster is unity (each cluster has a single BCG). Generating this figure requires understanding the effects of the angular resolution on the X-ray peak locations obtained with ROSAT as well as how to incorporate the high-resolution Chandra data into the sample. As it happens, however, this is only a minor issue as the ROSAT data are used mainly at larger radii and the Chandra data are used exclusively at small radii.

The solid points in Figure 11 represent the entire Xray sample, using both Chandra and ROSAT together, as radial bins of $r_{x}$. The innermost bin extends from the origin to $50 \mathrm{kpc}$, thus enclosing most of the ROSAT PSF. Subsequent bins are rings starting at $50 \mathrm{kpc}$, with inner and outer limits increasing geometrically by a factor of two. The data follow a simple power-law, which as we discuss below, appears to be the best form for the overall BCG spatial distribution. A log-log line fitted to the points gives the surface density as

$$
\begin{gathered}
\log _{10} \rho\left(r_{x}\right)=(-2.33 \pm 0.08) \log _{10}\left[\frac{r_{x}}{1 \mathrm{kpc}}\right] \\
+5.30 \pm 0.19 \log _{10}\left[\mathrm{~N} \mathrm{Mpc}^{-2}\right]
\end{gathered}
$$

which is shown in the figure as the red line.

We used Monte Carlo simulations to understand the effects of the limiting ROSAT resolution on the apparent density profile, and to verify that the profile incorporating ROSAT data was consistent with the center of the distribution inferred from Chandra alone. For an assumed surface-density profile, we drew $10^{4}$ "BCGs" for each cluster in the X-ray subset. For clusters observed by only by ROSAT, we scaled the angular position-error Gaussian to the appropriate physical resolution, given the redshift of the cluster, and drew a point at random from the circularly symmetric Gaussian centered at a radius drawn from the density distribution. For Chandra, we simply drew galaxies directly from the assumed profile. The form we tested included an inner quadratic-core to suppress the singular number integral as $r_{x} \rightarrow 0$, implied by the $\gamma<-2$ power-law,

$$
\rho\left(r_{x}\right)=\rho_{0}\left(1+\left(\frac{r_{x}}{a}\right)^{2}\right)^{\gamma / 2}
$$

where $a$ specifies the core scale, and $\rho_{0}$ the central surface density.

We did not attempt to derive $a$ formally, but simply compared the quality of the fits obtained by varying $a$ geometrically in the sequence of $a=5,10,20$, and 40 kpc. We fixed $\gamma=-2.33$, given its excellent description of the outer profile, where the ROSAT PSF would have little effect. As it happens, even with the large $50 \mathrm{kpc}$ outer limit of the inner-most bin using the full X-ray sample, we required $a \leq 10 \mathrm{kpc}$ to obtain a satisfactory fit to the central point. The "observed" profile for $a=$ $10 \mathrm{kpc}$ incorporating the blurring of the ROSAT offsets is shown in Figure 11 as the solid blue line, while the intrinsic unblurred-profile is shown as the dotted line. The effects of the ROSAT resolution is evident on the simulated profile, but with $a=10 \mathrm{kpc}$, the form given by equation (5) just matches the central point. It is also noteworthy that the profile incorporating ROSAT data is indeed compatible the profile inferred from Chandra data alone.

The subset of Chandra observations underscores the conclusion that any core in the BCG $r_{x}$ distribution must be extremely small. Figure 11 shows the implied surface density from the Chandra clusters alone. Again the Chandra points were incorporated into the bins representing the full sample, but here we can use considerably finer radial bins, given the superb Chandra angular resolution. The inner Chandra bin extends from the origin to $4 \mathrm{kpc}$, with the next two bins covering 4-16 and 16$64 \mathrm{kpc}$. The inner-most Chandra bin is fully an order of magnitude above the density implied by the $a=10$ kpc profile. The pure power-law fit given in equation (4) did not incorporate the central Chandra-only points, but its inward extrapolation clearly falls only slightly above them. In short, the BCG $r_{x}$ distribution shows no sign of any core or decrease in slope as $r_{x} \rightarrow 0$.

\subsubsection{Some BCGs Have Large X-ray Offsets}

A radial integral over equation (4) gives the cumulative distribution of the BCGs away from the cluster X-ray center, which is shown as an insert in Figure 11. The integral starts at $1 \mathrm{kpc}$ to avoid the central divergence, and continues out to include a few clusters with $r_{x}>1 \mathrm{Mpc}$. The steep power-law form in equation (4) unifies two superficially different pictures of where BCGs are located in their hosting clusters. The median $r_{x}$ implied by this distribution is only $\sim 10 \mathrm{kpc}$, and is consistent with the common impression that most BCGs reside close to the center of the X-ray gas, and presumably to the center of the cluster potentials. At the same time, the distribution also includes BCGs with large displacements from the X-ray defined center; $15 \%$ of the BCGs in the present sample have $r_{x}>100 \mathrm{kpc}$, with the largest offsets reaching $\sim 1 \mathrm{Mpc}$. Because the finding that some BCGs may be greatly displaced from the center of the cluster potential is strongly at odds with the paradigm that BCGs should be centrally located (at least in relaxed clusters), we review the evidence for BCGs with large $r_{x}$ and their import for understanding the formation of BCGs and clusters.

As noted in the Introduction, the first large survey of the X-ray morphology of galaxy clusters (Jones \& Forman 1984) showed that the majority of systems have well-defined X-ray cores largely coincident with the position of a bright galaxy, typically the BCG. However, the same study also showed that if the cluster sample was sorted by X-ray core radius, the ensemble showed a smooth progression to clusters with large X-ray cores not coincident with any particular galaxy. To underscore this point we note two well-studied rich clusters that have long been known to have BCGs markedly displaced from the peak of the X-ray emission. A1367, part 
of the present sample, is among the first examples found of a cluster with regular X-ray morphology, but with a large offset between the BCG (NGC 3842 in this case) and X-ray center (Bechtold et al. 1983); Table 7 gives $r_{x}=354 \mathrm{kpc}$ for this cluster. The Coma cluster (A1656) is the classic example of a rich galaxy cluster, yet it also is a system with a large BCG/X-ray offset, having $r_{x}=256$ kpc. White et al. (1993) analyzed a deep ROSAT image of Coma, and in conjunction with the positions and X-ray morphology of its two bright central elliptical galaxies, NGC 4889 (the BCG) and NGC 4874 (M2), concluded that Coma was produced in a still ongoing merger of two massive clusters.

Martel et al. (2014) emphasize that the BCG offset from the cluster center of mass (which may be different from the location of the peak X-ray emission), as well as the velocity offset discussed in the previous section, is a signature of the assembly of galaxy clusters by hierarchical merging. The BCG itself may be introduced into the cluster as part of an infalling group. A key point is that the galaxies, X-ray gas, and the dark matter halo of the cluster all have strongly different mechanisms and time scales for relaxing after cluster mergers. While we might expect the X-ray morphology of the cluster to be disturbed by strong or recent mergers with smaller clusters or groups, it is likely that the regularity of the X-ray gas distribution is re-established before any new BCG introduced by the merger is dynamically "captured" by the central potential. The regularity of the X-ray morphology plus the amplitude of $\left|\Delta V_{1} / \sigma_{c}\right|$ and $r_{x}$ in fact may provide means to constrain the recent merger history of clusters.

Previous studies of the location of BCGs with respect to the peak of the X-ray emission have produced diverse results. Patel et al. (2006) measured $r_{x}$ for a sample of 49 clusters and found $r_{x}>100 \mathrm{kpc}$ in 16 systems, or $33 \%$ of the sample, a fraction considerably larger than the $15 \%$ that we found. The positional accuracy of their centers is low, so a large fraction of the measured offsets with $r_{x} \sim 100 \mathrm{kpc}$ may really be significantly smaller; however their $r_{x}$ distribution has a long tail extending to three clusters with $r_{x}>500 \mathrm{kpc}$. In contrast, Haarsma et al. (2010) find $r_{x}>100 \mathrm{kpc}$ for just one cluster out of their small sample of 33, although, as we noted above, they also included proximity to the X-ray peak as a criterion for selecting their BCGs in the first place. It does appear that there is an important distinction between searching broadly within the cluster for the BCG versus selecting the brightest galaxy within the core of the $\mathrm{X}$ ray emission. Hashimoto et al. (2014) explicitly limited their search for the BCG to within $500 \mathrm{kpc}$ of the X-ray peak, but found offsets out to this limit.

While we have used extensive imaging and velocity observations to cast a wide net for the BCG in any cluster, we have relied on the literature to provide the matching X-ray centers. In order to understand the reliability of the largest BCG/X-ray offsets seen in our sample, we obtained archival Chandra or ROSAT images for the subset of clusters with $r_{x}>500 \mathrm{kpc}$. Of the clusters with X-ray centers available, we initially identified 29 clusters with $r_{x}>500 \mathrm{kpc}$. Of these, we accepted 22 clusters as credible systems with $r_{x}$ of this amplitude. Our criterion was that the X-ray center had to fall within the extended Xray source closest to the BCG that was associated with galaxies consistent with the cluster redshift.

Of the seven clusters rejected, three were cases in which the X-ray emission was from either a foreground or background system seen in projection close to the nominal cluster, which itself had no detectable X-ray emission. Since we thus had no valid X-ray center, these clusters were dropped from the set with X-ray data. In four clusters, the clusters were either binary, with the BCG clearly associated with a different X-ray component than we had assumed, or the X-ray emission from a projected cluster at different redshift had been selected over the $\mathrm{X}$-ray emission from the nominal cluster. In these cases we remeasured the $r_{x}$ with respect to the revised centers. In one cluster, A0548, $r_{x}$ decreased, but still remained $>500 \mathrm{kpc}$. In the end we conclude that $22 / 174$ or $12 \%$ of the sample has $r_{x}>500 \mathrm{kpc}$. We show X-ray maps for four examples of clusters with $r_{x} \sim 1 \mathrm{Mpc}$ in Figure 12 The clusters have well-defined central X-ray emission, but their BCGs are well outside of it.

In addition to these observational tests, we are encouraged by the cluster formation simulations of Martel et al. (2014), which produce ensembles of clusters that exhibit both the large $\left|\Delta V_{1} / \sigma_{c}\right|$ and $r_{x}$ seen in the present sample. Martel et al. argue that their simulations support cluster formation by the "merging group scenario." As various groups merge with the cluster over the age of the Universe, the identity of the BCG may change several times. Newly arrived BCGs can be marked by high $\left|\Delta V_{1} / \sigma_{c}\right|$ and large offsets from the center of their clusters, which we characterize with $r_{x}$.

The history of many of the more massive clusters simulated by Martel et al. (2014) show significantly long periods during which the BCG lies at a projected distance of more than $500 \mathrm{kpc}$ or even $1 \mathrm{Mpc}$ from the cluster center. The typical value of $\left|\Delta V_{1} / \sigma_{c}\right|$ is found to range over 0.15 to 0.31 for Abell-like clusters, in excellent agreement with the median value of 0.26 found for our sample in the previous section. The maximum value of $\left|\Delta V_{1} / \sigma_{c}\right|$ seen in the simulations may briefly exceed 1.5 in the early stages of a merger, again in good agreement with the observational limits on the BCG peculiar velocities. We argue later in this paper that additional lines of evidence support the merging-group scenario.

\section{THE PHOTOMETRIC AND KINEMATIC PROPERTIES OF BCGS}

The average luminosities of BCGs have long been known to have relatively little dispersion, allowing these galaxies to be used as "standard candles" (Humason et al. 1956: Sandage 1972a b). In this section we will explore the luminosity distribution function of BCGs and its relationship to other physical properties of the galaxies, such as their concentration and central stellar velocity dispersion. The BCG metric luminosities and structural parameters are tabulated in Table 7. The CMB frame has been assumed for calculation of all parameters.

\subsection{The Metric Luminosity of BCGs}

Figure 13 shows the distribution of $M_{m}$ for the present sample, where we have applied the extinction and $\mathrm{k}$ corrections outlined in $\$ 2.3 .2$ to the observed $R_{C}$ surface photometry. The distribution is well fitted by a Gaussian with mean $-22.844 \pm 0.016$, and standard deviation of $\sigma_{L}=0.337 \mathrm{mag}$. This is good agreement with 

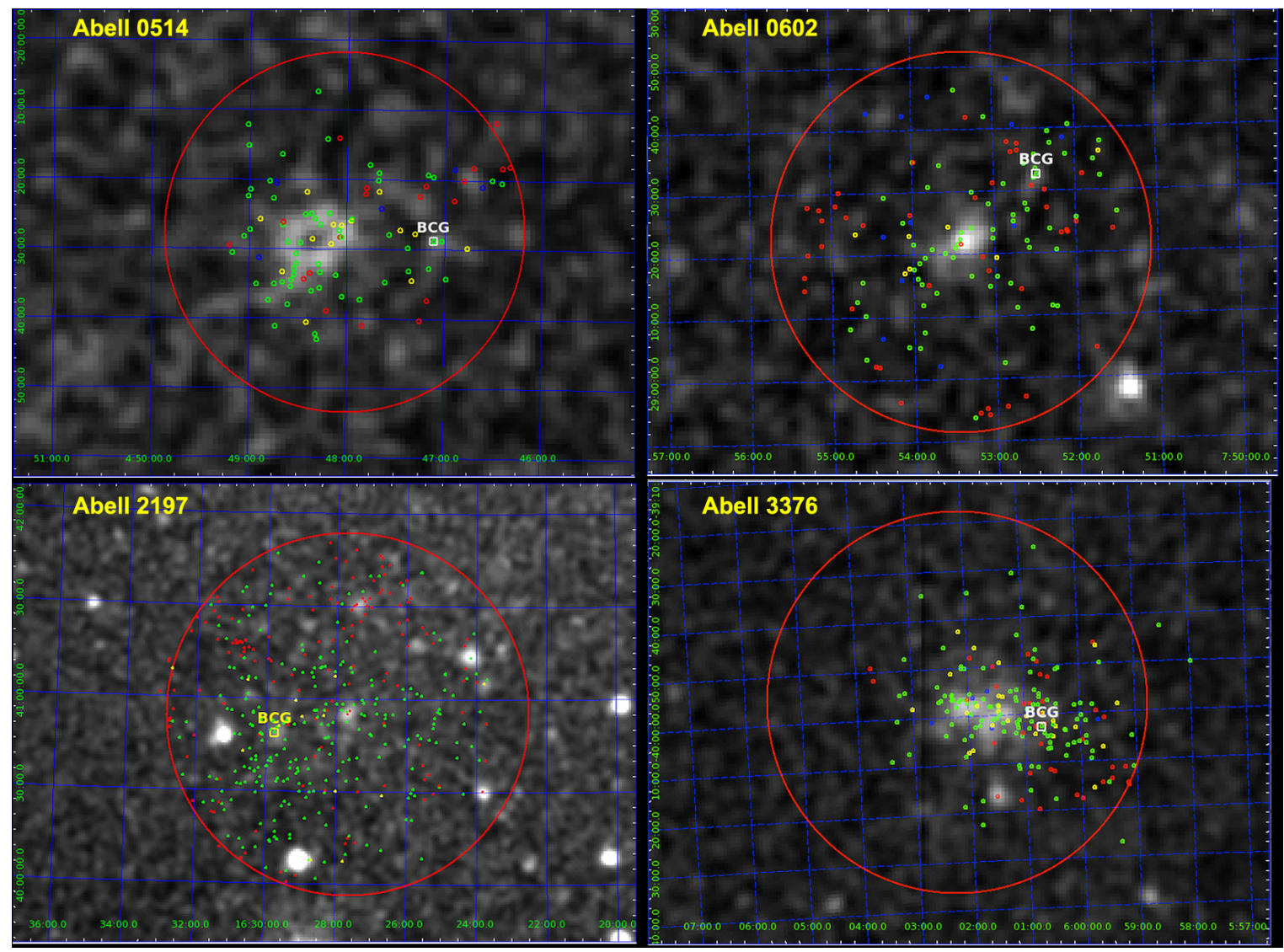

Figure 12. ROSAT All-Sky Survey images (smoothed with a gaussian) are shown for four clusters with $r_{x} \sim 1 \mathrm{Mpc}$. For A0514 $r_{x}=1060$ $\mathrm{kpc}$, A0602 $r_{x}=1134 \mathrm{kpc}$, A2197 $r_{x}=886 \mathrm{kpc}$, and A3376 $r_{x}=995 \mathrm{kpc}$. The large circle marks the Abell radius centered on the peak of the X-ray emission. The location of the BCG is indicated. Points mark galaxies with measured velocities, with green for galaxies within $1000 \mathrm{~km} \mathrm{~s}^{-1}$ of the mean cluster velocity, yellow for galaxies within 1000 to $1500 \mathrm{~km} \mathrm{~s}^{-1}$ of the mean, red for galaxies with velocities $>1500 \mathrm{~km} \mathrm{~s}^{-1}$ above the mean, and blue for galaxies with velocities $>1500 \mathrm{~km} \mathrm{~s}^{-1}$ below the mean. In all four clusters the BCG clearly falls within the velocity and spatial distributions of cluster galaxies, but are markedly displaced from peak of the associated X-ray emission.

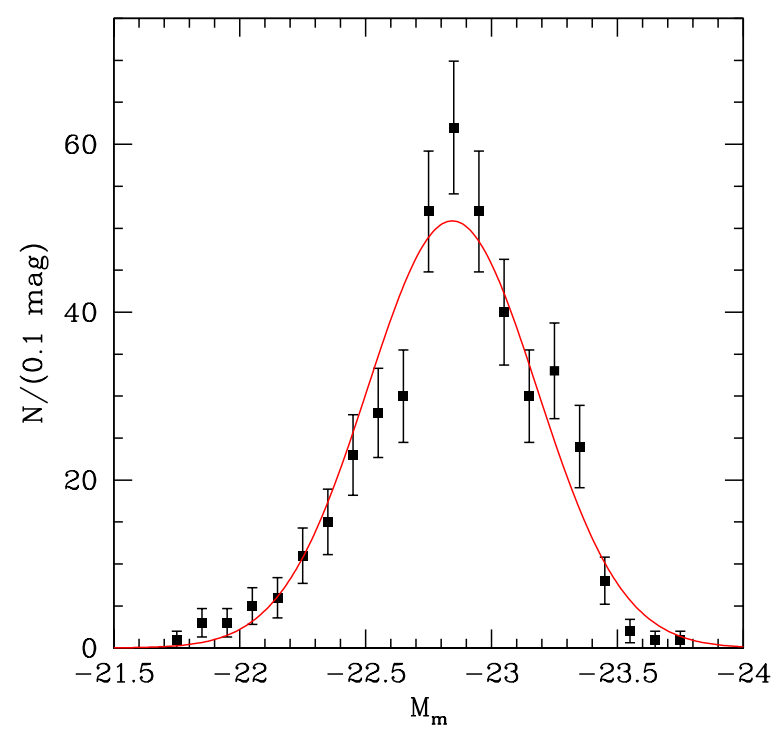

Figure 13. The binned distribution of metric luminosity, $M_{m}$, for the present BCG sample. The bins are 0.1 mags wide and the errors are from Poisson statistics. The red line shows the best-fit Gaussian, which has mean $M_{m}=-22.844 \pm 0.016$ and $\sigma_{L}=0.337$ $\operatorname{mag}\left(R_{C}\right.$ band)

$\sigma_{L}=0.327$ mag measured from the $15 \mathrm{~K}$ sample in PL95.
We have excluded three extremely faint BCGs of the total sample of 433 BCGs from the Gaussian fit and most of the analysis that follows. The lowest luminosity bin plotted in Figure 13 is $-21.7>M_{m}>-21.8$, which contains a single BCG. ${ }^{9}$ The three BCGs in question, those in A3188, A3599, and A3685, all have $M_{m}>-21.46$, which is yet fainter by $\sim \sigma_{L}$; all are fainter than the mean $M_{m}$ by $>4 \sigma_{L}$. A3599 and A3685 have very few cluster members with velocities and may not be real systems.

\subsection{The $L_{m}-\alpha$ Relationship}

Hoessel (1980) observed a sample of BCGs to refine their use as "standard candles" in cosmological probes, finding that $L_{m}$ correlated with the physical concentration of the galaxies. Elliptical galaxies have long been known to have a relationship between total luminosity and effective radius, which is reflected in the relationship between the metric luminosity and radial scale as well. Hoessel (1980) expressed the physical concentration of the BCGs in terms of $\alpha$, the logarithmic slope of the variation of $L_{m}$ with the physical radius of the aperture, $r$, evaluated at the metric radius:

$$
\alpha \equiv d \log L_{m} /\left.d \log r\right|_{r_{m}} .
$$

${ }^{9}$ When we explicitly refer to $L_{m}$ in magnitude units we will use the variable $M_{m}$, or absolute metric magnitude. 


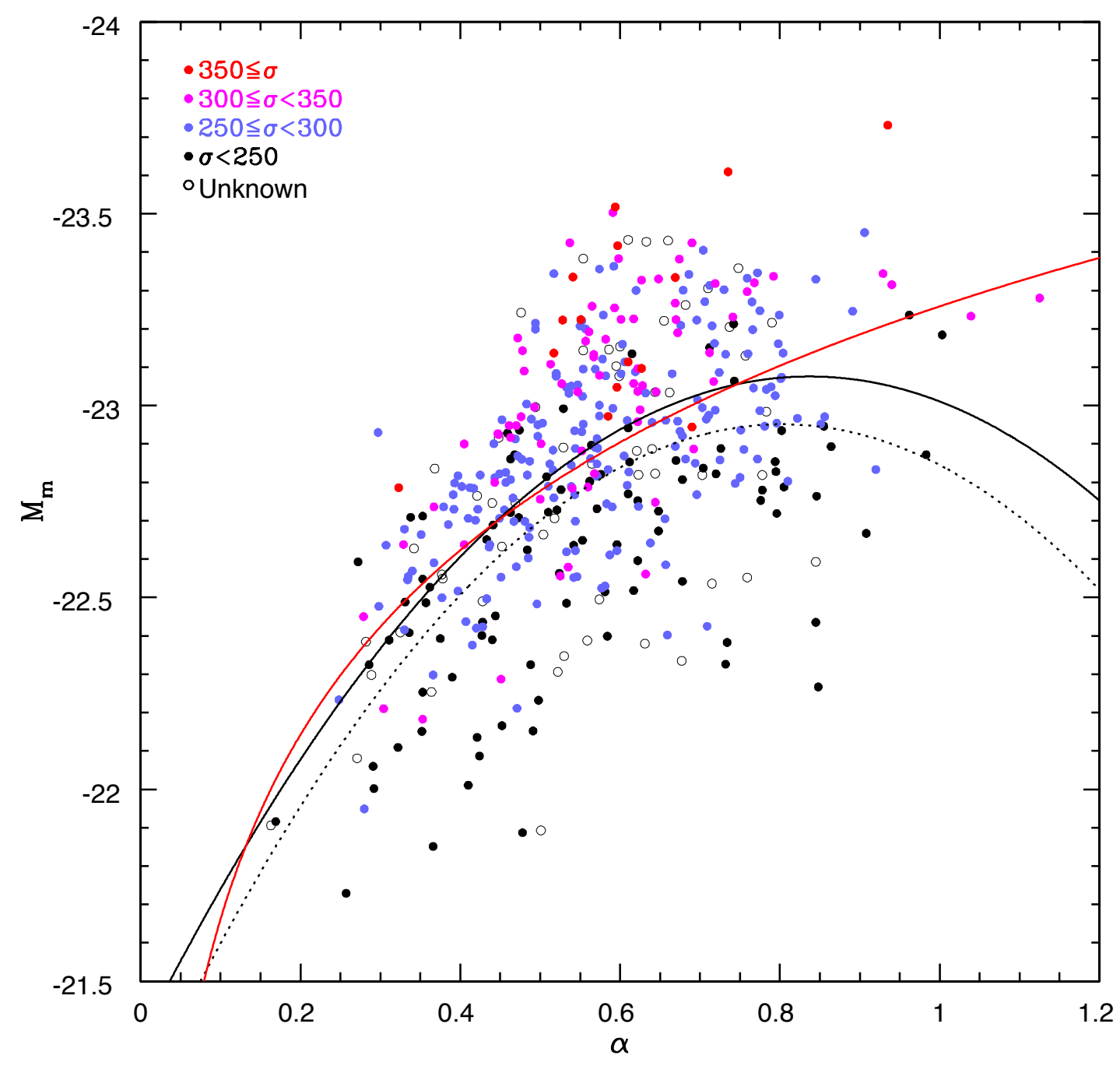

Figure 14. The relationship between metric luminosity, $M_{m}$, and $\alpha$ is plotted for BCGs. The solid black line is the mean quadratic $L_{m}-\alpha$ relation for the present sample, given by equation $\sqrt{7}$, while the red line fits the present sample with a linear function of log $\alpha$ (equation 8). The dotted line shows the quadratic $L_{m}-\alpha$ relation of PL95 rescaled to $H_{0}=70 \mathrm{~km} \mathrm{~s}^{-1} \mathrm{Mpc}^{-1}$. Symbols are color-coded by central stellar velocity dispersion, $\sigma$. Note that at any $\alpha$, objects with higher $M_{m}$ typically have higher $\sigma$.

In addition to serving as a measure for the concentration of the BCGs, $\alpha$ also relates the error in $L_{m}$ to a corresponding distance error, $\sigma_{D}=\sigma_{L} /(2-\alpha)$ for using BCGs as standard candles. When $\alpha=0$, all the galaxy light is contained within the aperture, and $L_{m}$ is the total luminosity, while when $\alpha=2$, the surface brightness distribution is constant with radius, and the metric luminosity provides no information on distance.

The $L_{m}-\alpha$ relationship observed by Hoessel (1980) showed that $\alpha$ initially increases steeply with $L_{m}$, then plateaus for the more luminous BCGs. LP94 and PL95 confirmed this behavior, and quantified it by fitting a quadratic relationship between $L_{m}$ and $\alpha$, which they used as a distance indicator. For this application, $\alpha$ is used to predict $L_{m}$, which in turn is used as a "standard candle" to infer the distance to the BCGs - in this case, $L_{m}$ must be the dependent, rather than independent variable. The $L_{m}-\alpha$ relationship for the present sample is plotted in Figure 14. A quadratic form fitted to all BCG but the three with $M_{m}>-21.5$ gives

$$
M_{m}=-21.35 \pm 0.13-(4.12 \pm 0.43) \alpha+(2.46 \pm 0.36) \alpha^{2} .
$$

We note that we weight all points equally in this fit, and those that follow, unless we indicate otherwise. This is because the observed scatter around the relation of Eq. 7 is over an order of magnitude larger than the formal observational errors. The residuals about the relation are $0.267 \mathrm{mag} \mathrm{rms}$ in $M_{m}$ in the CMB frame, essentially identical to the CMB-frame residuals of $0.261 \mathrm{mag}$ for the $15 \mathrm{~K}$ sample of LP94. In passing, we note that the quadratic form gives significantly smaller residuals in $L_{m}$ than does a simple linear relation in $\alpha$, as we first showed in PL95. The bulk flow of the Abell cluster sample with respect to the CMB was derived by LP94 by finding the average BCG peculiar velocity field that minimized residuals in the $L_{m}-\alpha$ relationship.

The quadratic form of the $L_{m}-\alpha$ relationship derived 


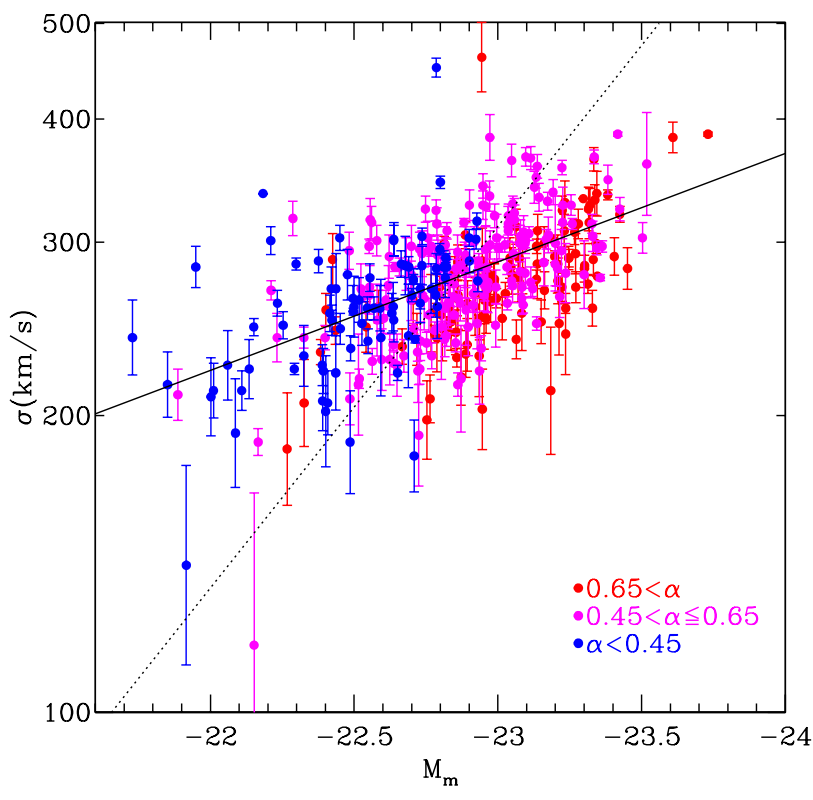

Figure 15. Central stellar velocity dispersion, $\sigma$, is plotted as a function of metric luminosity, $L_{m}$. The solid line is the mean relationship between the two parameters when $L_{m}$ is the independent variable (equation 9). The dotted line is the relationship fitted when $\sigma$ is the independent variable (equation 10. The points are color-coded by $\alpha$. Note that at any $\sigma, \alpha$ tends to increase with $L_{m}$.

by PL95 is also shown in Figure 14 . The earlier relation falls $\sim 0.1 \mathrm{mag}$ below the present relationship. The majority of this offset (0.06 mag) is likely to be due to our present use of Schlegel et al. (1998) extinctions rather than the Burstein \& Heiles (1982) values used by PL95; the average $A_{B}$ values from SFD are 0.10 mag greater than those of $\mathrm{B} \& \mathrm{H}$ for the $15 \mathrm{~K}$ sample.

The quadratic form has the unattractive feature, however, of reaching maximum $L_{m}$ at $\alpha \sim 0.8$, and then predicting fainter $L_{m}$ as $\alpha$ increases beyond this point where there are few BCGs to elucidate its behavior. This motivated us to introduce a new form, fitting $L_{m}$ to a linear function of $\log \alpha$. This new form closely parallels the quadratic form over most of the domain, but is monotonic, and may better represent the handful of BCGs with $\alpha>0.9$. This form has the additional advantage of requiring only two, rather than three parameters. For the present sample with $M_{m}<-21.5$, we measure

$$
M_{m}=-23.26 \pm 0.03-(1.597 \pm 0.104) \log _{10} \alpha .
$$

The residuals in $M_{m}$ are $0.271 \mathrm{mag}$ rms in the $\mathrm{CMB}$ frame, only 0.004 mag larger than those for the form given in equation (7). This form is also plotted in Figure 14 and only strongly differs from the quadratic form for $\alpha>1$, where we have very few galaxies.

\subsection{The $L_{m}-\sigma$ Relationship}

Figure 15 plots the relationship between $M_{m}$ and $\sigma$. While the measurement errors in $\sigma$ are subdominant to the intrinsic scatter in this relationship, they are not completely negligible, and we have incorporated them into our fitting procedure, following the methodology described in Hogg et al. (2010) and Kelly (2011).

If $M_{m}$ is treated as the independent variable, the relationship derived from a simple least-squares fit for the

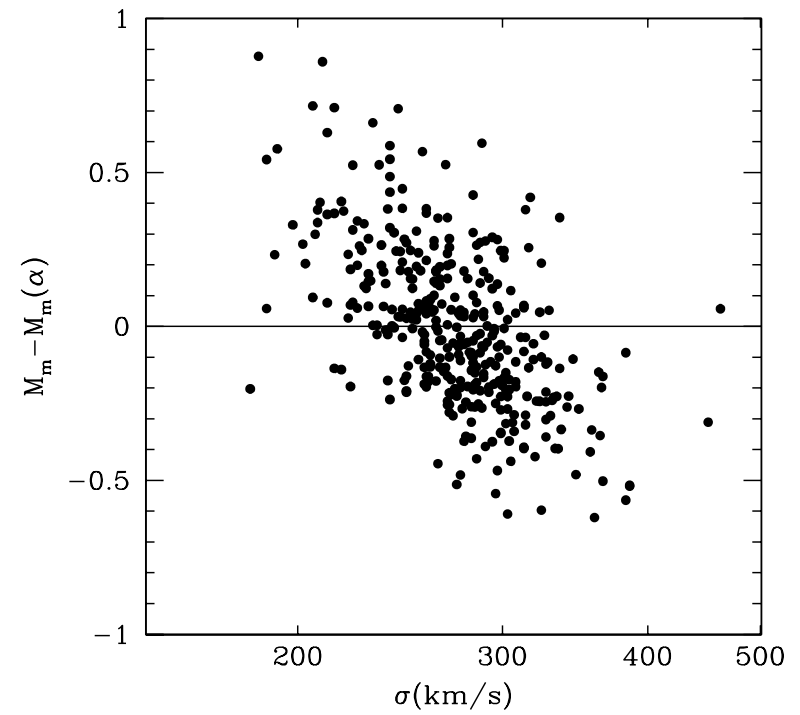

Figure 16. Residuals in $M_{m}$ from the mean relationship between $M_{m}$ and $\alpha$ given by equation $(8)$ and shown in Figure 14 are plotted as a function of central stellar velocity dispersion, $\sigma$. A clear correlation is evident in the sense that positive residuals (BCGs with fainter than the mean $M_{m}$ for a given $\alpha$ ) correspond to low $\sigma$ and negative residuals correspond to higher values of $\sigma$.

369 galaxies with $M_{m}<-21.5$ and measured $\sigma$ is:

$$
\begin{aligned}
& \log _{10}\left(\frac{\sigma}{300 \mathrm{~km} \mathrm{~s}^{-1}}\right)= \\
& \quad-(0.275 \pm 0.023)\left(\frac{M_{m}}{2.5}\right)-2.55 \pm 0.21
\end{aligned}
$$

with $0.052 \pm 0.02$ scatter in $\log \sigma$, corresponding to $\pm 12 \%$ in $\sigma$. Conversely, if $\sigma$ is the independent variable, then

$$
\begin{array}{r}
M_{m}=-22.956 \pm 0.015-2.5(1.09 \pm 0.08) \times \\
\log _{10}\left(\frac{\sigma}{300 \mathrm{~km} \mathrm{~s}^{-1}}\right),
\end{array}
$$

with an intrinsic scatter of $0.278 \pm 0.011$ in $M_{m}$, implying that $\sigma$ is just as good as $\alpha$ is for predicting $M_{m}$.

The slope in equation $(9)$ is essentially the same as the classic Faber \& Jackson (1976) result of $\sigma \propto L^{1 / 4}$ for normal elliptical galaxies. This suggests that the central portions of the BCGs enclosed within $r_{m}$ may have a "normal" relationship between $\sigma$ and $L$, in contrast to that between total BCG L and $\sigma$. As noted in the Introduction, Oegerle \& Hoessel (1991) and Lauer et al. (2007) found that $\sigma$ is only weakly correlated with BCG total luminosity. This behavior may reflect the putative formation of BCGs by dry mergers of less luminous elliptical galaxies. Simulations of this process shows that $\sigma$ remains essentially constant over dry mergers, with the effective radius, $R_{e}$, growing rapidly with $L$ (BoylanKolchin et al. 2006). This associated steepening of the $R_{e}-L$ relation has also been seen in BCGs (Lauer et al. 2007 ).

We also fitted the Faber-Jackson relationship with $M_{m}$ measured at $2 \times$ and $4 \times$ the nominal metric radius to test the hypothesis that the relation between BCG $L$ and $\sigma$ becomes shallower as $r_{m}$ increases to include a larger fraction of total galaxy luminosity, When the metric ra- 


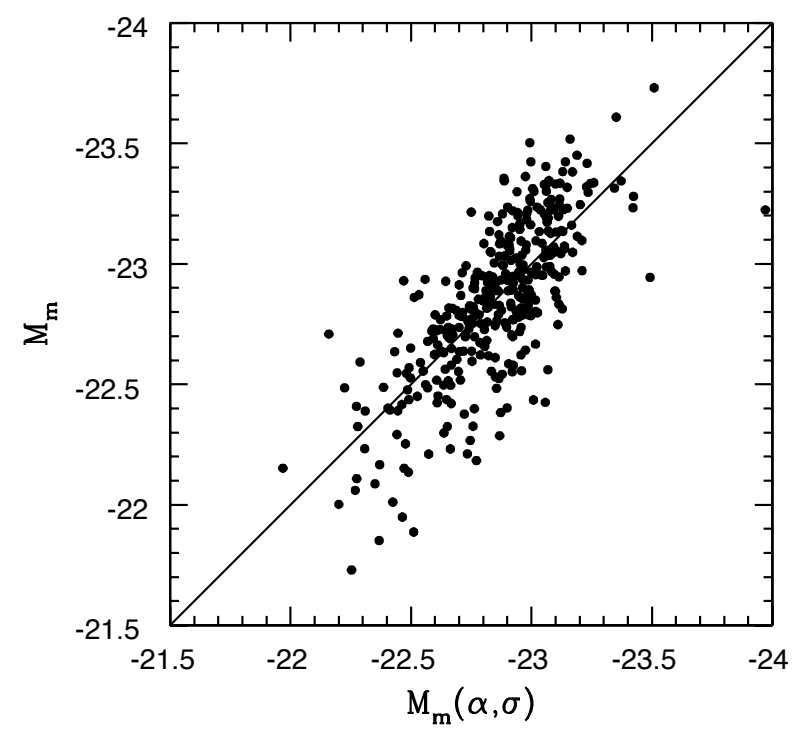

Figure 17. Metric luminosity is plotted as a function of $M_{m}$ estimated from $\alpha$ and $\sigma$ through the multi-parameter relationship between the three parameters given by equation (13).

dius is doubled, we find

$$
\begin{aligned}
& \log _{10}\left(\frac{\sigma}{300 \mathrm{~km} \mathrm{~s}^{-1}}\right)= \\
& -(0.205 \pm 0.022) M_{m}\left(2 r_{m}\right) / 2.5-1.94 \pm 0.20,
\end{aligned}
$$

for the 352 BCGs that have both valid $\sigma$ and photometry at $2 r_{m}$, with an intrinsic scatter of $0.054 \pm 0.02$ in $\log \sigma$. For $M_{m}$ measured at $4 r_{m}$ the sample decreases to 200 BCGs, and we measure

$$
\begin{aligned}
& \log _{10}\left(\frac{\sigma}{300 \mathrm{~km} \mathrm{~s}^{-1}}\right)= \\
& -(0.147 \pm 0.027) M_{m}\left(4 r_{m}\right) / 2.5-1.44 \pm 0.26,
\end{aligned}
$$

with an intrinsic scatter of $0.053 \pm 0.03$ in $\log \sigma$. In short, the slope decreases from $\sim 1 / 4$ to $\sim 1 / 5$ and then $\sim 1 / 6$ as $r_{m}$ is doubled twice. As even at $4 r_{m}$ the integrated luminosity of the BCGs is still increasing, the relationship for $\sigma$ and total luminosity would be yet shallower.

\subsection{The $L_{m}-\alpha-\sigma$ "Metric Plane"}

With the large $24 \mathrm{~K}$ sample and improved knowledge of galaxy and cluster parameters over what was available to PL95, we can now better investigate sources of residual scatter in the $L_{m}-\alpha$ or $L_{m}-\sigma$ relationships. In fact, given the fundamental plane relationships between total $L, \sigma$, and $R_{e}$ (Dressler et al. 1987; Djorgovski \& Davis 1987) for ordinary elliptical galaxies, it is not surprising to find that a multi-parameter "metric plane" relationship between $L_{m}, \sigma$, and $\alpha$, has smaller scatter than those between any two of these parameters.

The points in the $L_{m}-\alpha$ relationship shown in Figure 14 are color-coded by $\sigma$, showing a strong gradient such that at any $\alpha$, higher $L_{m}$ is correlated with higher $\sigma$. Likewise, the color-coding of the points by $\alpha$ in the $L_{m}-$ $\sigma$ plot in Figure 15 show that at any $\sigma$, higher $L_{m}$ is correlated with higher $\alpha$. This behavior is shown more explicitly in Figure 16, which plots the $M_{m}$ residuals from the mean $L_{m}-\alpha$ relationship given by equation
(8), as a function of $\sigma$. A strong correlation is clearly evident.

Use of $\alpha$ and $\sigma$ together to predict $M_{m}$ for the 368 galaxies with $M_{m}<-21.5$ and measured $\sigma$ gives the relationship,

$$
\begin{aligned}
M_{m}=- & 23.31 \pm 0.03-(1.43 \pm 0.09) \log _{10} \alpha \\
& -(2.20 \pm 0.17) \log _{10}\left(\frac{\sigma}{300 \mathrm{~km} \mathrm{~s}^{-1}}\right) .
\end{aligned}
$$

The intrinsic scatter is $0.214 \pm 0.010$ in $M_{m}$, a marked improvement over the $L_{m}-\alpha$ and $L_{m}-\sigma$ relationships. Figure 17 plots observed $M_{m}$ as a function of $M_{m}(\alpha, \sigma)$ estimated from this relationship. This metric plane is not identical to a fundamental plane relation, but with $L_{m}$ and $\alpha$ serving as proxies for $L$ and $R_{e}$, it encodes similar structural information. A detailed comparison between the metric plane and a true fundamental plane relation for the present sample is the subject of our second paper (Chisari et al. 2014).

\subsection{The Relationship Between BCGs and Their Clusters \\ 4.5.1. BCGs and the Bulk Properties of Clusters}

As noted in the Introduction, the structure and luminosity of BCGs may be tied to the properties of the clusters as traced by the temperature and luminosity of the associated X-ray emitting gas. The present $24 \mathrm{~K}$ sample suggests that BCG luminosity is also correlated with cluster velocity dispersion, which can serve as a proxy for cluster X-ray luminosity, $L_{X}$, given the relationship between the two cluster parameters (Solinger \& Tucker 1972; Quintana \& Melnick 1982, Wu et al.1999, Mahdavi \& Geller 2001).

Figure 18 plots cluster velocity dispersion as a function of both $M_{m}$ and $\alpha$ for the 259 clusters having 25 or more galaxy redshifts, the minimum number needed for accurate measurement of the velocity dispersion. While there is considerable scatter in the velocity dispersion at any $M_{m}$ or $\alpha$, there is a clear correlation such that the median dispersion increases by nearly a factor of two over the range of both parameters. The relation between $\alpha$ and $\sigma_{c}$ in particular echoes the relation between the shallowness of the BCG surface brightness profile and cluster X-ray luminosity seen by Schombert (1988) and Brough et al. (2005).

Both correlations seen in Figure 18 raise the question of whether or not $\sigma_{c}$ offers any independent information that can reduce some of the scatter in the $L_{m}-\alpha$ relationship. Hudson \& Ebeling (1997) argued that there is an $L_{m}-\alpha-L_{X}$ relationship, which offers better prediction of $L_{m}$ than using $\alpha$ alone and in doing so reduces the significance of the LP94 bulk-flow amplitude. If we take $\sigma_{c}$ as a proxy for $L_{X}$, we should thus expect to see a $L_{m}-\alpha-\sigma_{c}$ relationship if the Hudson \& Ebeling (1997) is highly significant.

Figure 19 plots the residuals of the $L_{m}-\alpha$ relationship, given by equation (8), as a function of cluster velocity dispersion. The residuals of the $L_{m}-\alpha$ relationship do show a barely significant correlation with cluster velocity dispersion in the sense that brighter residuals in $L_{m}$ are still associated with clusters with higher velocity dispersion. The measured slope is $-0.23 \pm 0.10 \mathrm{mag}$ per dex in dispersion, such that the mean $M_{m}$ residuals decrease 


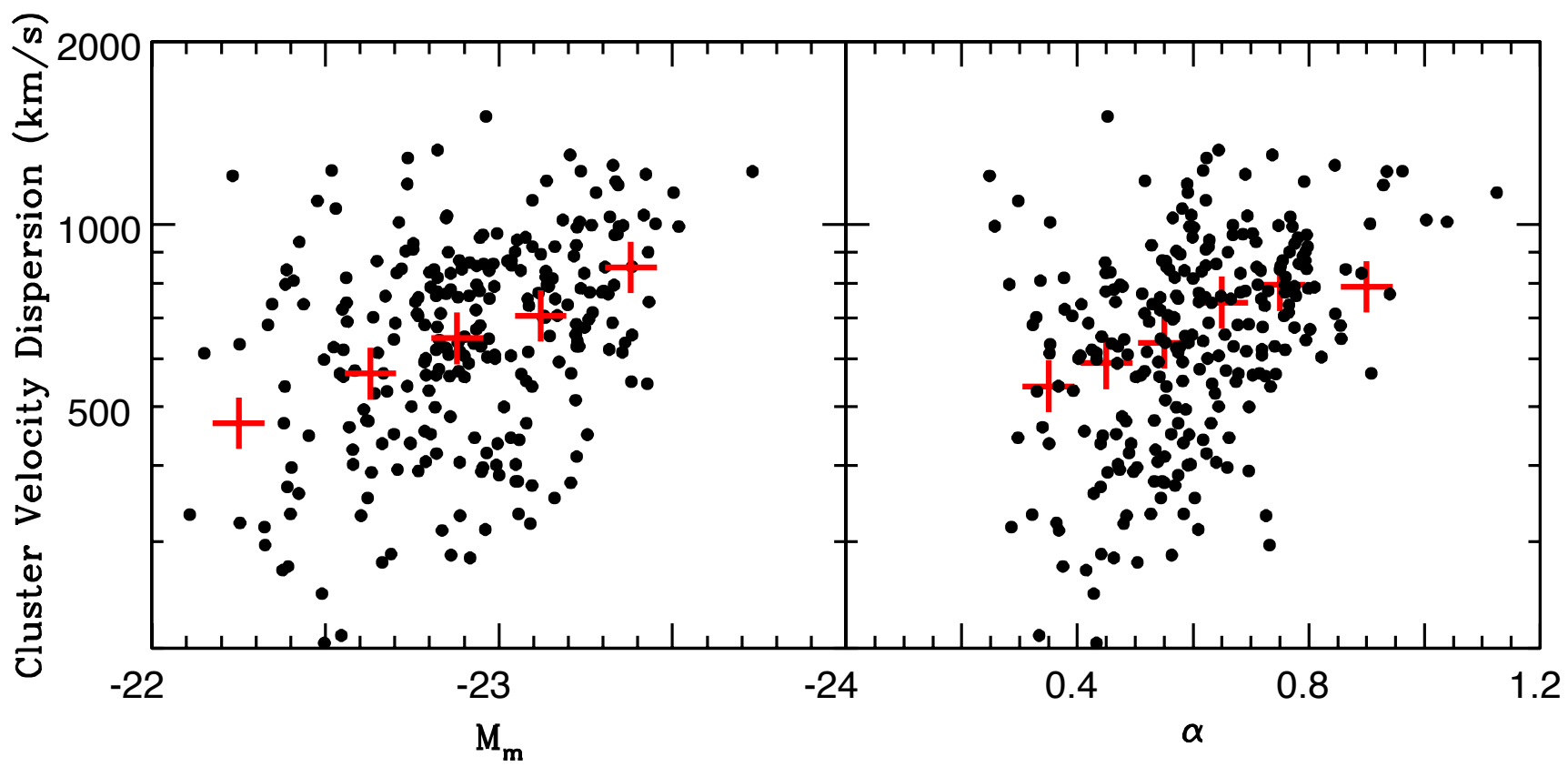

Figure 18. Cluster velocity dispersion is plotted as a function of BCG $M_{m}$ (left panel) and $\alpha$ (right panel) for the 259 clusters with 25 or more galaxy redshifts. The red crosses give the median velocity dispersion for each 0.25 mag in $M_{m}$ or 0.2 bin in $\alpha$. Both parameters increase with velocity dispersion.

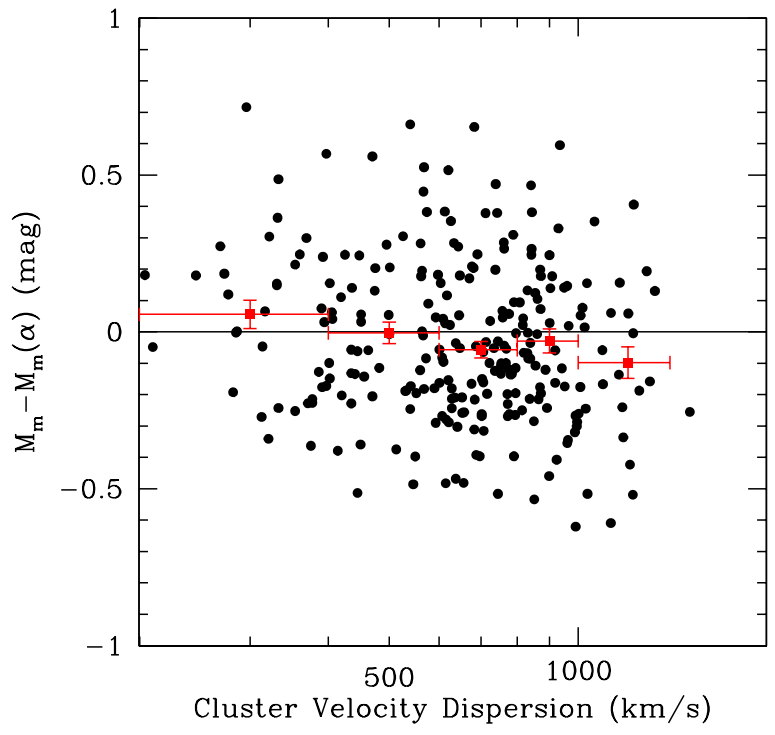

Figure 19. Luminosity residuals from the $L_{m}-\alpha$ relationship (equation 8) are plotted as a function of cluster velocity dispersion for the 259 clusters with 25 or more galaxy redshifts. The red points give the mean residual in bins of width $200 \mathrm{~km} \mathrm{~s}^{-1}$ (except for the highest bin, which was widened to include more points). The mean of the $M_{m}$ residuals decreases by $\sim 0.15$ mag over the range of the cluster velocity dispersions.

by $\sim 0.15$ mag over the sample range of cluster velocity dispersion.

The $L_{m}-\alpha-\sigma$ relation (equation 13 ), however, "soaks up" this residual dependence on cluster velocity dispersion. When $\alpha$ and $\sigma$ are used to predict $L_{m}$, the remaining dependence on cluster velocity dispersion decreases to $\sim 0.07 \mathrm{mag} / \mathrm{dex}$ with no significance over the sample range in dispersion. Notably, the $L_{m}$ residuals from this relation are already slightly better than those of the Hudson \& Ebeling (1997) $L_{m}-\alpha-L_{X}$ relationship. The present $L_{m}-\alpha-\sigma$ relation thus offers a BCG-based distance indicator with the effects of the cluster environment removed.

Both the metric luminosity and structure of the BCGs may also be related to the peculiar velocities of the BCGs within their hosting clusters. Figure 20 plots the peculiar velocities of the BCGs normalized by cluster velocity dispersion as a function of BCG $L_{m}$ and $\alpha$ for the subset of clusters with 25 or more members. The median peculiar velocity steadily decreases with increasing $\alpha$. The trend of median $\left|\Delta V_{1} / \sigma\right|$ with $M_{m}$ is less clear; however, the most luminous BCGs have relatively smaller peculiar velocities.

\subsubsection{The Structure of BCGs and Their Positions Within the Clusters}

Figure 21 shows that $\alpha$ is also correlated with the offset of the BCG within the cluster relative to the X-ray center. The right panel shows that $\alpha$ is clearly larger for BCGs closer to the center of their clusters, while left panel of the figure shows that $M_{m}$ is largely unrelated to the position of the BCG within the cluster. The former result appears to be consistent with the weak correlation discovered by Ascaso et al. (2011) between the effective radii of the BCGs and their spatial offsets, with larger BCGs being positioned closer to the centers of the clusters.

One possibility is that the increase of $\alpha$ may be dominated by high-speed or non-merging interactions with other galaxies in the cluster. The interactions would preferentially take place more often at the center of the clus- 


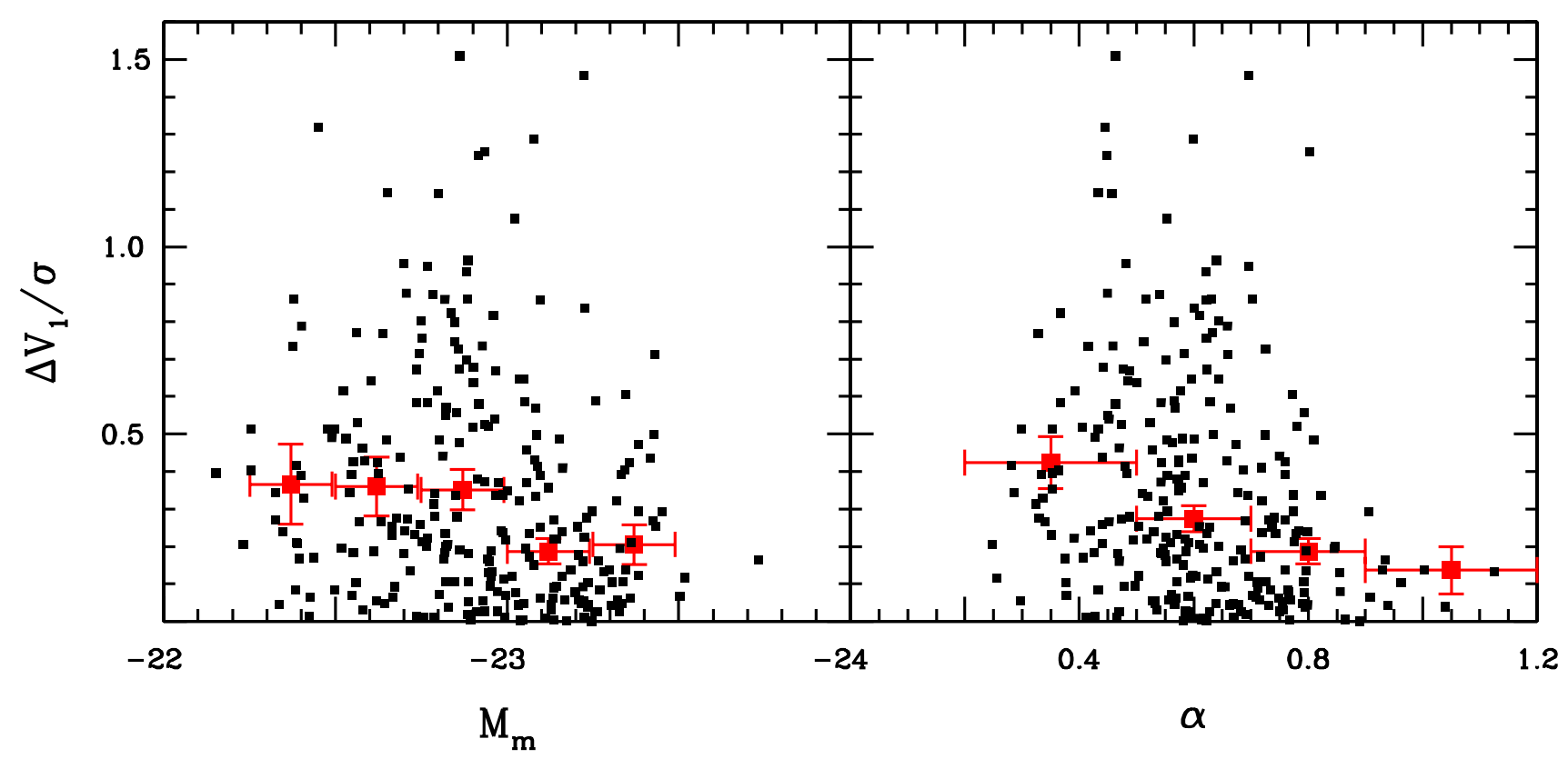

Figure 20. Absolute peculiar velocity of the BCGs normalized by cluster velocity dispersion is plotted as a function of BCG $M_{m}$ and $\alpha$ for the subset of clusters with 25 or more members. The red points gives the median $\left|\Delta V_{1} / \sigma\right|$ in 0.25 mag bins in $M_{m}$ or 0.2 bins in $\alpha$.
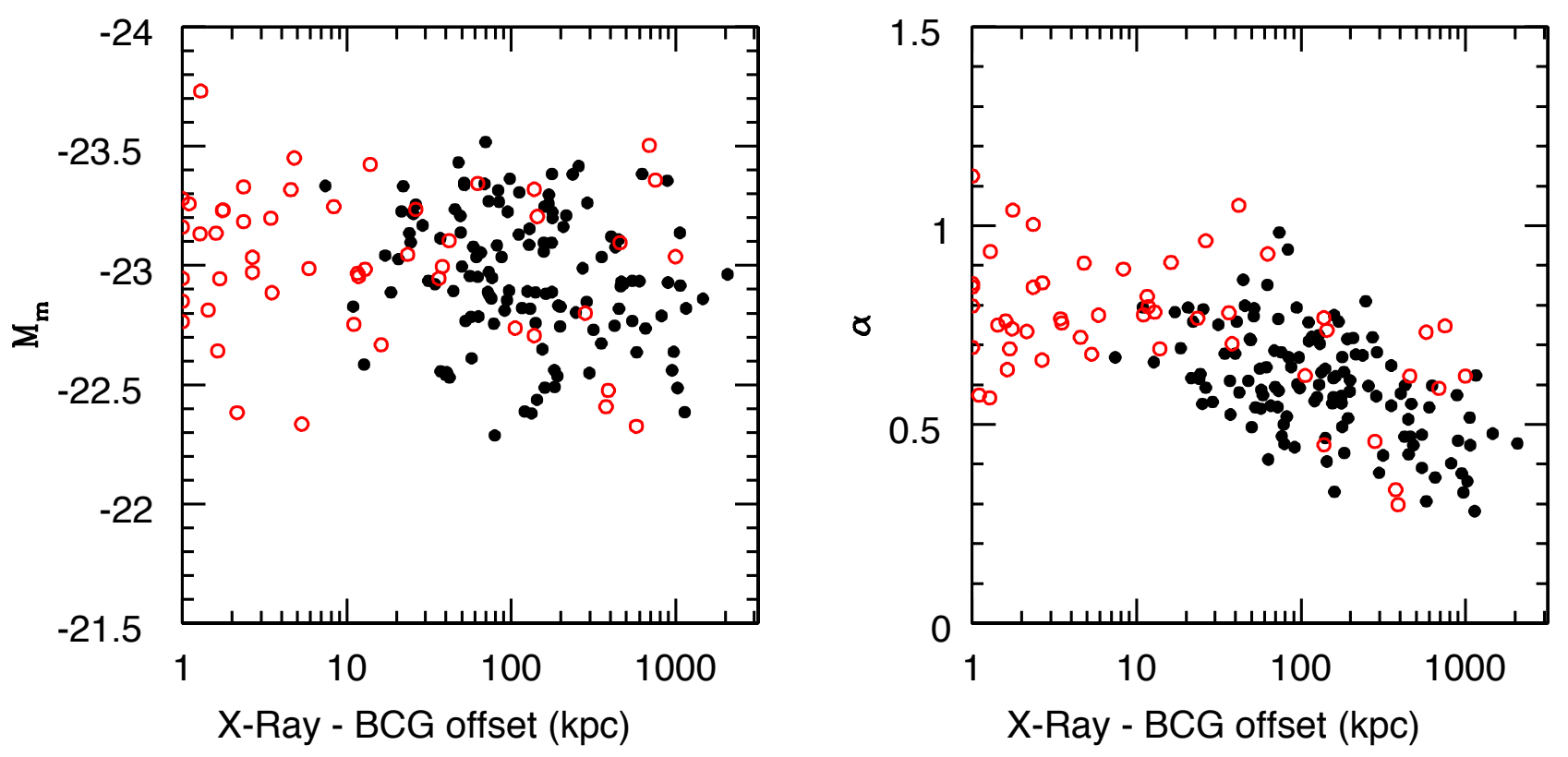

Figure 21. $M_{m}$ and $\alpha$ are plotted as a function of distance of the BCG from the X-ray defined center of the cluster. Solid black symbols indicate clusters with ROSAT measurements, while the open red symbols indicate clusters with Chandra-based X-ray centers. Little dependence on $M_{m}$ with distance from the center is seen, while $\alpha$ increases with decreasing distance. 


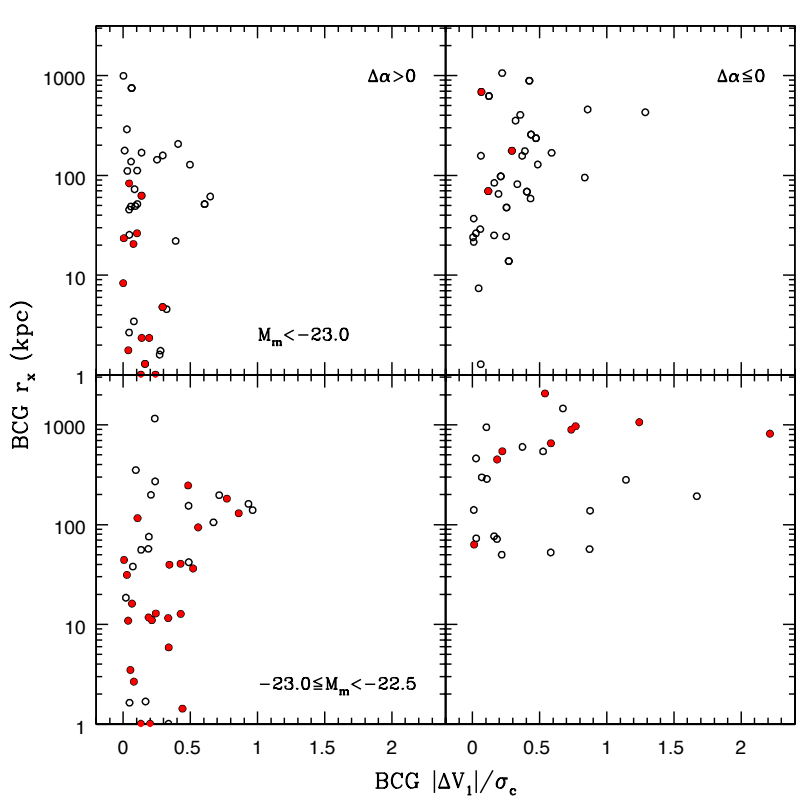

Figure 22. The distribution of BCG X-ray offset, $r_{x}$, and normalized velocity offset, $\left|\Delta V_{1}\right| / \sigma_{c}$, (for clusters with $N \geq 25$ velocities) are shown for four regions in the BCG $M_{m}-\alpha$ space. The columns separate BCGs by whether or not they fall above (left) or below (right) the mean relation of $\alpha$ on $M_{m}$ (equation 14). The rows then segregate the BCGs by $M_{m}$ interval, with the brightest BCGs plotted in the top row. Red points are BCGs with $\alpha$ deviating from the mean $\alpha\left(M_{m}\right)$ relationship by $1 \sigma$ or more.

ter and would add energy to the stellar envelope of the BCGs, causing them to become more extended. Without actual mergers, however, little stellar mass is added to the BCGs, thus no overall luminosity growth occurs as the structure of the BCGs becomes more extended. A second hypothesis is that merging does occur as the BCG dwells within the cluster center, but the density of stars within the metric aperture does not increase in the process. Hausman \& Ostriker (1978) argued that dry mergers in fact would cause little central growth of the BCGs, a phenomenon that is also seen in merger simulations (Boylan-Kolchin et al. 2006). Recent theoretical and observational work (Hopkins et al. 2009, van Dokkum et al. 2010) indeed argues that growth of massive galaxies since redshifts $\sim 2$ is mainly in their outer envelopes.

Since both $L_{m}$ and $\alpha$ are related to the position of the BCGs with respect to both the spatial and velocity centroids of the clusters, we also examined the combined effect of the last two parameters. Figure 22 revisits the plot of the BCG spatial location within the cluster, $r_{x}$, versus the normalized absolute BCG peculiar velocity, $\left|\Delta V_{1}\right| / \sigma_{c}$, which was first shown for the full sample of BCGs with X-ray centers and accurate mean velocities in Figure 10. We now split the BCGs with $M_{m}<-22.5$ into two luminosity bins, ${ }^{10}$ each of which is split further into two halves by whether or not the galaxies are above or below the mean relation for $\alpha$, given $M_{m}$,

$\alpha\left(M_{m}\right)=(-0.256 \pm 0.019)\left(M_{m}+22.5\right)+0.484 \pm 0.009$.

10 There are very few BCGs with $M_{m}>-22.5$ that have X-ray cluster centers available, thus we cannot do this analysis for the lower-luminosity BCGs.

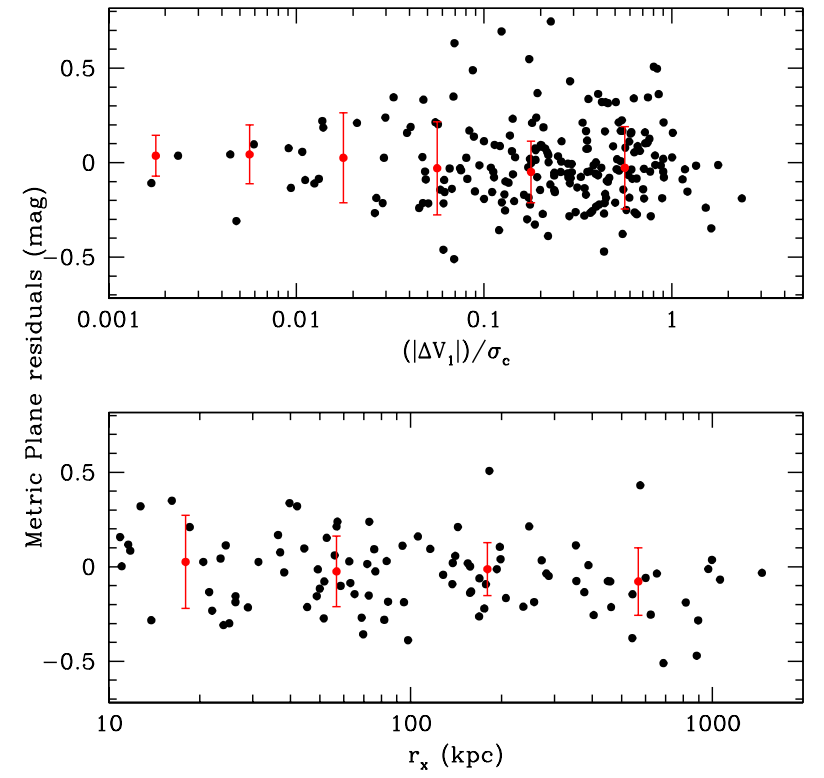

Figure 23. The magnitude residuals from the metric plane relation of Equation (13), shown as a function of velocity offset from the mean redshift of the cluster (upper panel) and positional offset from the X-ray center, where available (lower panel). As in Figures 8 and 22 the velocity offset is normalized by the velocity dispersion of the cluster. The median values in bins, and the one-sigma widths (as determined from the interquartile range) are also shown in red. There is no evidence for a systematic bias, or larger scatter, at large offset in either velocity or position.

These four subsets are shown as individual panels in Figure 22, with the columns separating BCGs with higher (left) or lower (right) than average $\alpha$, given $M_{m}$, and the rows corresponding to the two luminosity bins, with the brightest BCGs plotted in the top row. In any panel, we additionally note (red symbols) BCGs with $\alpha$ residuals in excess of the rms residual, $\sigma_{\alpha}$ about the mean relation; $\sigma_{\alpha}=0.13$ for the full BCG sample.

Examination of the individual panels suggests that $r_{x}$ and $\left|\Delta V_{1}\right| / \sigma_{c}$ indeed both work together to moderate the structure of the BCGs. The upper left panel in Figure 22 contains the most luminous $\left(M_{m}<-23.0\right)$ and extended BCGs. There are no BCGs with $\left|\Delta V_{1}\right| / \sigma_{c}>0.7$, and the BCGs with $\Delta \alpha>\sigma_{\alpha}$, have $\left|\Delta V_{1}\right| / \sigma_{c}<0.3$, or peculiar velocities less than half of those of the BCGs in this luminosity range with $0<\Delta \alpha<\sigma_{\alpha}$. Moreover, these large $\Delta \alpha$ galaxies have $r_{x}<100 \mathrm{kpc}$, while the BCGs with $0<\Delta \alpha<\sigma_{\alpha}$ can have $r_{x}$ an order of magnitude larger.

The galaxies in the upper right panel of Figure 22 are just as luminous as the BCGs in the panel to their left, however this subset now has three galaxies with $\left|\Delta V_{1}\right| / \sigma_{c}>0.7$, and a paucity of galaxies with $r_{x}<10$ kpc. The three galaxies with $\Delta \alpha<-\sigma_{\alpha}$ have $r_{x}$ an order of magnitude larger than those with $\sigma_{\alpha}<\Delta \alpha<0$.

BCGs in the $-23.0<M_{m}<-22.5$ luminosity bin largely echo the behavior exhibited by the more luminous BCGs, although the BCGs with $\Delta \alpha>\sigma_{\alpha}$ have $\left|\Delta V_{1}\right| / \sigma_{c}$ about twice as large. The BCGs in this luminosity range with $\Delta \alpha<\sigma_{\alpha}$ (right-middle panel) avoid the centers of their clusters even more, however, with no galaxies having $r_{x}<50 \mathrm{kpc}$. The BCGs with $\Delta \alpha<-\sigma_{\alpha}$ have $r_{x}$ an order of magnitude yet larger.

The overall picture, again, is that $\alpha$ is larger for the BCGs that lie closer to the spatial and velocity center 


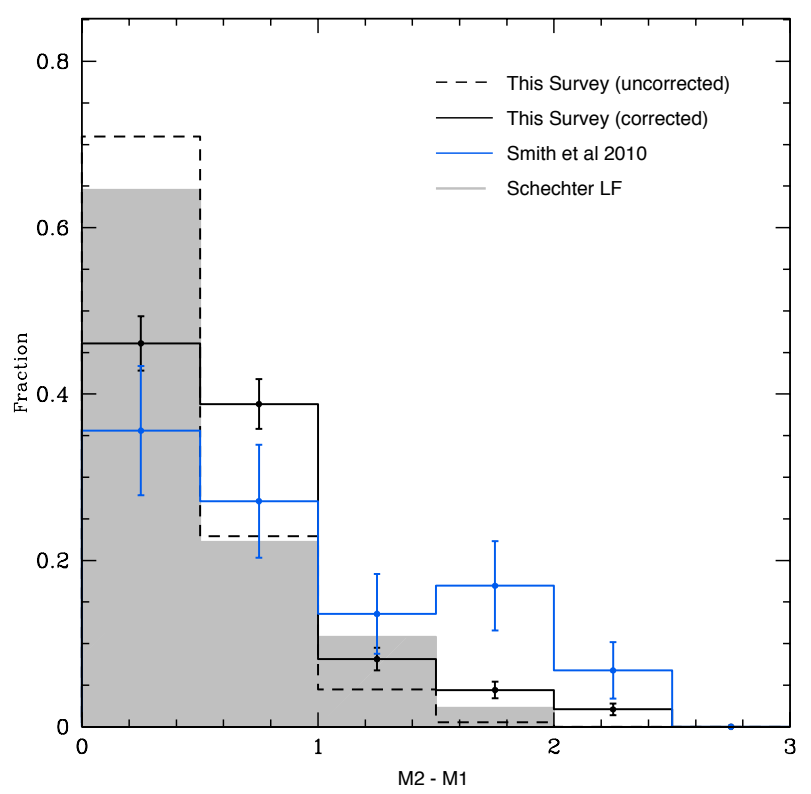

Figure 24. The distribution of $M_{2}-M_{1}$ for various cluster samples. The dashed black histogram shows the $M_{m}(\mathrm{M} 2)-M_{m}(\mathrm{BCG})$ distribution for the 179 clusters for which we directly observed both the BCG and the second-ranked galaxy. $M_{m}(\mathrm{M} 2)$ and $M_{m}(\mathrm{BCG})$ are the metric luminosities of the M2 galaxy and the BCG, respectively. The solid black line shows this distribution corrected using a reference set of 30 clusters with $M_{m}(\mathrm{M} 2)-M_{m}$ (BCG) derived from the SDSS. These 30 clusters were selected at random from amongst the clusters that we did not directly observe the secondranked galaxy. The solid blue histogram shows the $M_{2}-M_{1}$ distribution from the study done by Smith et al. (2010). The light grey shaded histogram shows the expected $M_{2}-M_{1}$ distribution for 2000 simulated clusters with galaxy luminosities drawn from a Schechter luminosity function.

of their hosting clusters. BCGs with $r_{x}<10 \mathrm{kpc}$ and $\left|\Delta V_{1}\right| / \sigma_{c}<0.5$ are highly likely to have markedly higher $\alpha$, with $\Delta \alpha>\sigma_{\alpha}$ as compared to BCGs of the same luminosity. Conversely, galaxies with $\left|\Delta V_{1}\right| / \sigma_{c}>1$ always have large $r_{x}$ and $\Delta \alpha<0$. It is striking that the BCGs with $r_{x} \sim 50 \mathrm{kpc}$ have larger $\alpha$ than do BCGs at larger distances from the center - these galaxies are still well displaced from the X-ray center, but yet are deep enough within the potential such that $\alpha$ has already been affected. Conversely, position within the cluster seems to have little effect on $L_{m}$, as we already saw in Figures 20 and 21

While $\alpha$ is dependent on the spatial and velocity locations of the BCGs within the cluster, the metric plane scatter seems to be independent of both. Figure 23 shows the residuals of the metric plane (equation 13 as a function of $\left|\Delta V_{1}\right| / \sigma_{c}$ (upper panel), and for those objects with $\mathrm{X}$-ray data, as a function of $r_{x}$ (lower panel). There is no evidence for a bias or increased scatter for objects with large offsets. The metric plane thus again implicitly accounts for the environmental effects of the clusters, regardless of whether the BCGs reside in the center of the cluster or in its outskirts.

\section{THE NATURE OF SECOND-RANKED GALAXIES}

\subsection{The Photometric Properties of M2}

We imaged the second-ranked galaxies, M2, in $~ 41 \%$ of the clusters in the total sample. As we described in 2.2 .1 we did this mainly when the identity of the BCG

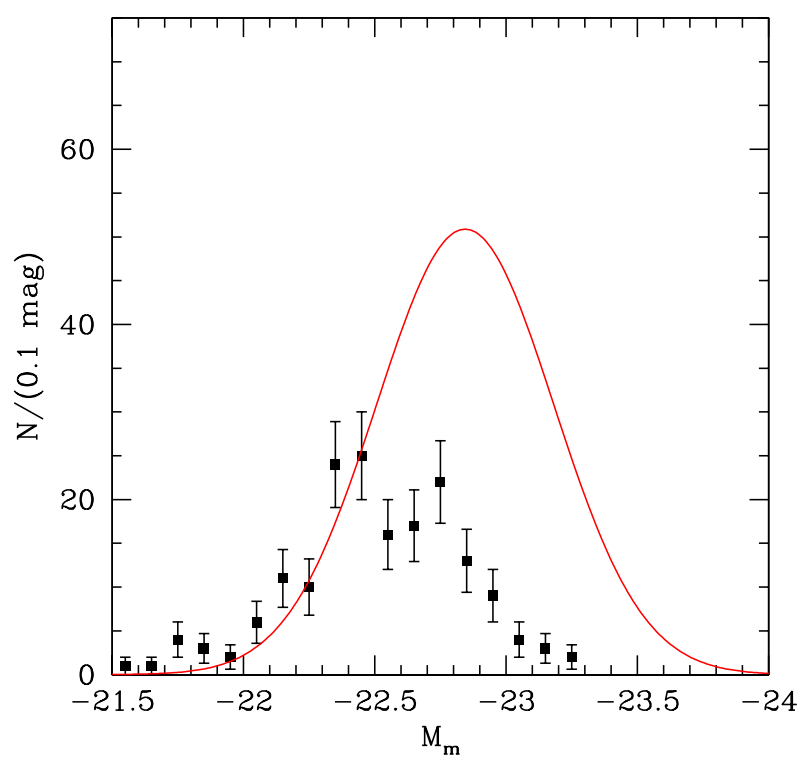

Figure 25. This figure shows the distribution of the metric luminosities of the observed M2 sample. The red line is the Gaussian fitted to the BCG luminosity function shown in Figure 13

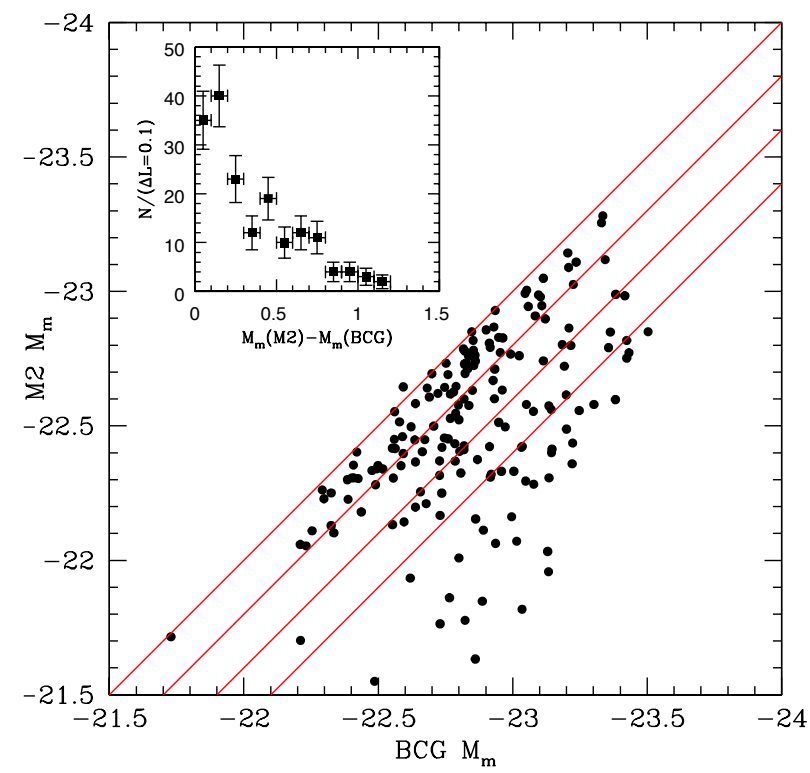

Figure 26. The distribution of $M_{m}(\mathrm{BCG})$ versus $M_{m}(\mathrm{M} 2)$, the metric luminosities of the $\mathrm{BCG}$ and $\mathrm{M} 2$ galaxies, respectively, is plotted for the clusters with M2 observations (41\% of the total sample). The upper red line line marks $M_{m}(\mathrm{BCG})=M_{m}(\mathrm{M} 2)$, with the subsequent lines marking offsets of $M_{m}(\mathrm{M} 2)$ from $M_{m}$ (BCG) in $0.2 \mathrm{mag}$ steps. The inset figure gives the histogram of the difference in metric magnitude between the BCG and M2 in finer bins than shown in Figure 24 This is equivalent to binning along the red lines in the main figure. (The one M2 above the line is that in A3531, where the BCG becomes the brightest galaxy in an aperture slightly larger than the nominal $r_{m}$.)

during the initial definition of the sample was ambiguous, thus we are less likely to have data on M2 in cases where the BCG is dominant, i.e., considerably brighter than M2. We assess how strongly our observational procedures bias our derived $M_{m}(\mathrm{M} 2)-M_{m}(\mathrm{BCG})$ distribution by randomly selecting 30 clusters in our $15 \mathrm{~K}$ sample for which we did not observe the second-rank galaxy. Here, 


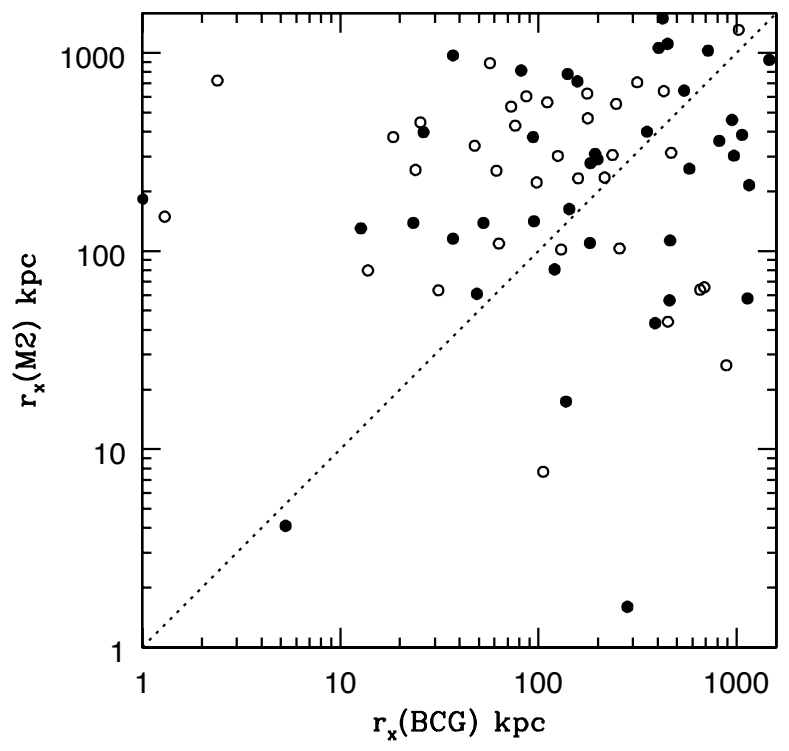

Figure 27. The projected offsets of M2 from the cluster X-ray centers are plotted against those of the BCG. Solid symbols are clusters for which $M_{m}(\mathrm{M} 2)-M_{m}(\mathrm{BCG})<0.3$ mag. while open symbols mark clusters with relatively less luminous M2s. Note that when $r_{x}(\mathrm{BCG})>100 \mathrm{kpc}$ there are only three clusters with a "rival" $\mathrm{M} 2$ with $r_{x}(\mathrm{M} 2)<50 \mathrm{kpc}$. Clusters with large $r_{x}(\mathrm{BCG})$ thus do not correspond to those with small $r_{x}(\mathrm{M} 2)$. The cluster in the figure with the smallest $r_{x}(\mathrm{M} 2)$ is A0539. Its BCG has a large peculiar velocity and a smaller $\alpha$ than its $\mathrm{M} 2$, but the BCG is brighter than M2 for all aperture radii.

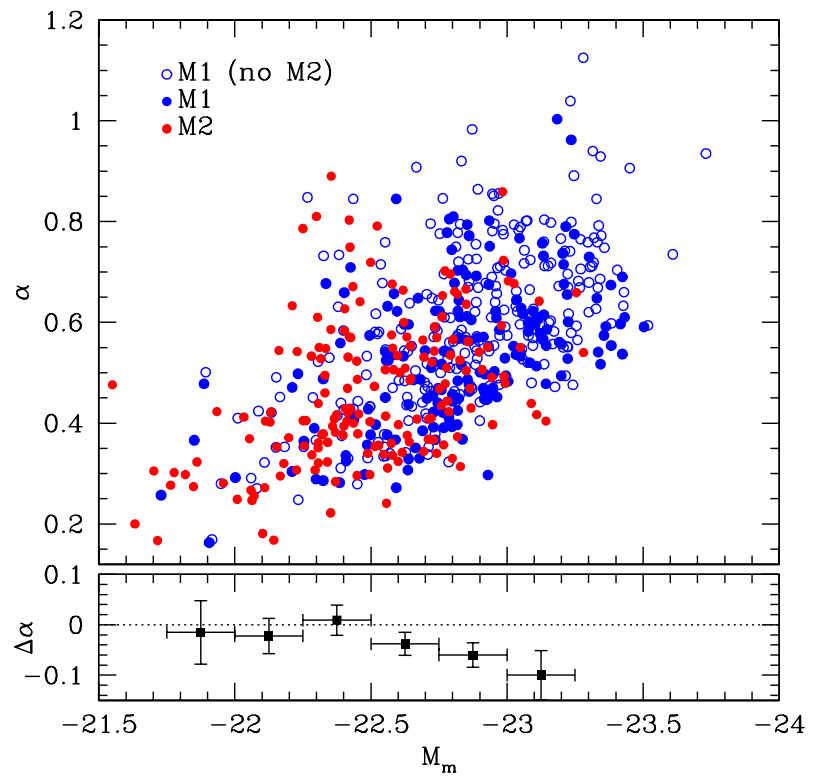

Figure 28. The correlation between $\alpha$ and $M_{m}$ for BCGs (blue; open symbols are BCGs in clusters lacking observed M2s) and M2s (red); $M_{m}$ is now shown as the independent variable. The mean difference between the BCGs and M2 $\alpha$ in each 0.25 mag bin in $M_{m}$ is plotted at the bottom. For $M_{m}>-22.5$, no difference is seen between the two populations, while the M2 have increasingly smaller average $\alpha$ than the BCGs as $M_{m}$ increases in luminosity over $M_{m}<-22.5$.

$M_{m}(\mathrm{M} 2)$ and $M_{m}(\mathrm{BCG})$ are the metric luminosities of the M2 galaxy and the BCG, respectively. For these 30 clusters, we used the Sloan Digital Sky Survey (SDSS) r-band images to derive $M_{m}(\mathrm{M} 2)-M_{m}(\mathrm{BCG})$ using the

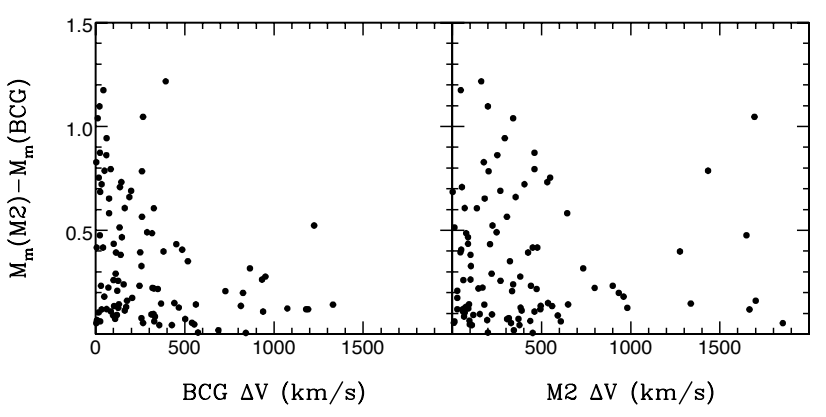

Figure 29. The left panel shows the M2-BCG luminosity difference as function of the absolute peculiar radial velocity of the BCG within the cluster. In the right panel the luminosity difference is plotted with respect to the peculiar velocity of M2. Only clusters with 25 members or more are plotted. Note that $M_{m}(\mathrm{M} 2)-M_{m}$ (BCG) $<0.5$ for nearly all clusters in which the BCG $\Delta V_{1}>400 \mathrm{~km} \mathrm{~s}^{-1}$.

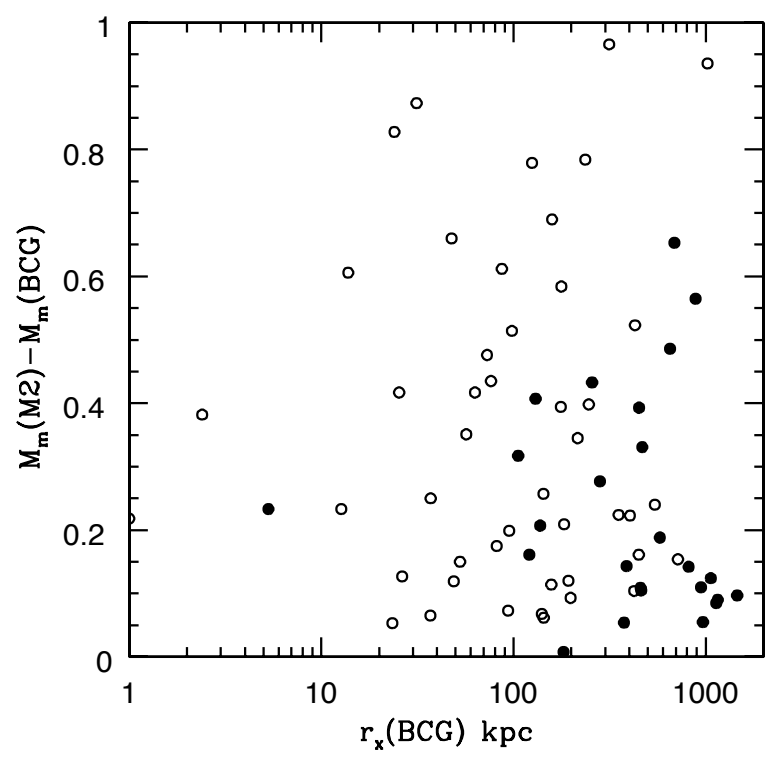

Figure 30. The luminosity difference between M2 and their matching BCG, $M_{m}(\mathrm{M} 2)-M_{m}(\mathrm{BCG})$ is plotted as a function of the offset of the BCG from the X-ray center. Solid symbols are clusters for which the M2 falls closer than the BCG to the Xray center, while open symbols mark the opposite case. There is no relationship between the luminosity of M2 and the BCG until $r_{x}<20 \mathrm{kpc}$, where no M2s with $M_{m}(\mathrm{M} 2)-M_{m}(\mathrm{BCG})<0.2 \mathrm{mag}$ are seen. This figure ratifies the impression from Figure 27 that there are very few clusters with M2 closer to the X-ray center once the BCG $r_{x}<100 \mathrm{kpc}$.

same photometric techniques as described in $\$ 2.3 .2$. Under the assumption that this randomly selected sample of 30 clusters is representative of the clusters for which we did not initially observe the second-ranked galaxy, we derive a corrected $M_{m}(\mathrm{M} 2)-M_{m}(\mathrm{BCG})$ distribution by drawing $M_{m}(\mathrm{M} 2)-M_{m}(\mathrm{BCG})$ values from the SDSS sample for the remaining $\sim 59 \%$ of the clusters in our sample. The results are shown in Figure 24. The clusters for which we did observe M2 in our survey have a $M_{m}(\mathrm{M} 2)-M_{m}(\mathrm{BCG})$ distribution that is peaked at lower values than the corrected $M_{m}(\mathrm{M} 2)-M_{m}(\mathrm{BCG})$ distribution. We show, for comparison, the $M_{2}-M_{1}$ distribution from Smith et al. (2010) and from galaxies drawn from 2000 realizations of clusters with a Schechter 


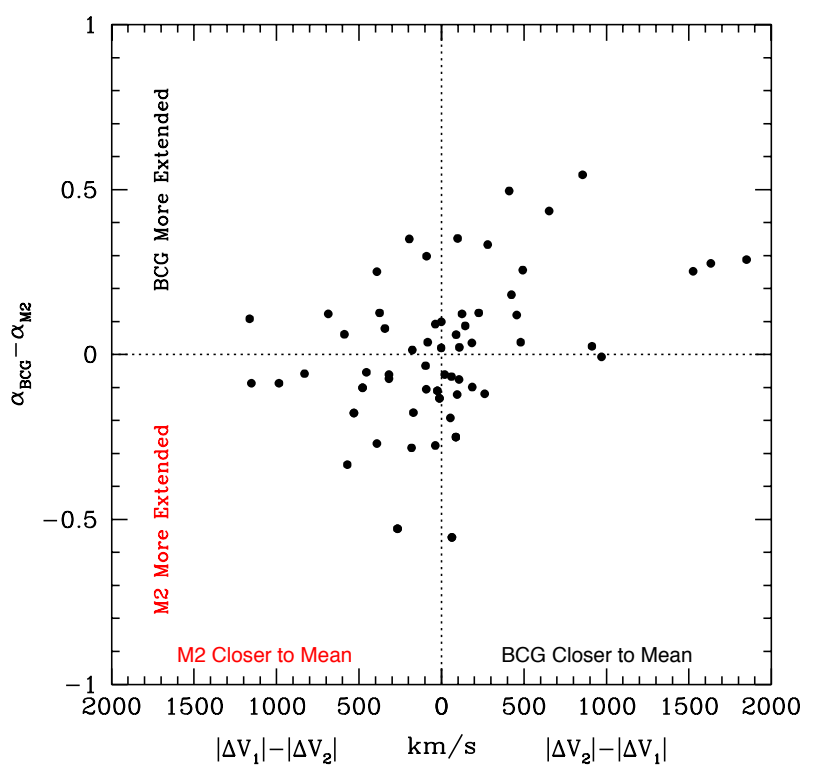

Figure 31. The difference in $\alpha$ between the BCG and M2 in a given cluster is plotted as a function of the difference between the absolute values of their peculiar velocities relative to the mean cluster velocity, for M2 galaxies with $M_{m}(\mathrm{M} 2)-M_{m}(\mathrm{BCG})<0.3$ mag and clusters having 25 or more galaxy velocities. When the M2 falls closer to the mean velocity, typically $\alpha_{2}>\alpha_{1}$, while the opposite is true when the BCG is the galaxy closer to the mean velocity.

(1976) luminosity function. A KS test rejects consistency between our corrected $M_{m}(\mathrm{M} 2)-M_{m}(\mathrm{BCG})$ distribution and that from the Smith et al. (2010) study at the $99 \%$ confidence level. Our selection procedure clearly introduces a significant bias that cannot be completely compensated for. However, the Smith et al. (2010) clusters are an X-ray selected sample, and thus may favor more luminous BCGs; their corresponding M2s may thus follow a different luminosity-offset distribution from ours as well. While this rules out performing analyses that require a complete distribution of $M_{m}(\mathrm{BCG})$ vs. $M_{m}(\mathrm{M} 2)$, we can, however, compare the structure of the BCG and M2 on a per-cluster basis. It also appears that our M2 sample is close to complete for $M_{m}(\mathrm{M} 2)-M_{m}(\mathrm{BCG}) \leq 0.3$ mag.

Figure 25 shows the distribution of the metric absolute magnitude, $M_{m}$, of the present M2 sample compared to the Gaussian representation of the BCG luminosity function shown in Figure $13{ }^{11}$ Interestingly, the largest differences between the $\overline{M 2}$ and BCG distributions are seen at the bright end, rather than the fainter end, where one might expect the selection effects to be the most severe. There are few highly luminous M2s, despite a deliberate effort to include such galaxies in the sample. For example, while there are 139 BCGs that have $M_{m}<-23$, there are only nine M2s that exceed this threshold.

\subsection{The Relationship of M2 versus the BCG in the Hosting Cluster}

Figure 26 plots the BCG versus M2 luminosities for each cluster, as well as a histogram of the difference in the metric magnitude between the BCG and M2 in finer

\footnotetext{
11 All the M2 parameters plotted in the figures in this section are provided in Table 8
}

bins than shown in Figure 24. In the majority of cases, the M2 systems imaged are within a few tenths of a magnitude of the BCGs. Indeed, the M2 in any given cluster may be more luminous than many of the BCGs in other clusters. We observed M2s in 54 of the 139 clusters with BCGs with $M_{m} \leq-23$, or $39 \%$ of the systems, a fraction essentially identical to that for all clusters. At the same time, only $17 / 54$ or $\sim 30 \%$ of this subset have M2s within 0.3 mag of the BCG $M_{m}$, a level at which we consider the M2 to be a close "rival" of the BCG, while $60 / 98$ or $\sim 60 \%$ of the BCGs with M2s observed and $-23<M_{m} \leq-22.5$ have an M2 that is a close rival. The difference between the two subsets is readily evident in Figure 26. This result is consistent with the classic result from Sandage \& Hardy (1973) that the most luminous BCGs are associated with relatively faint M2s. The large luminosity differences between the BCG and M2 for the brightest BCGs are also consistent with the arguments of Tremaine \& Richstone (1977), Loh \& Strauss (2006), and Lin et al. (2010) that these galaxies are "special" and are not drawn from a standard luminosity function.

Figure 27 shows that the projected offsets of the BCGs and their corresponding M2s from the X-ray center of their clusters follow different distributions. There are essentially no M2s closer to the X-ray center than the BCG once $r_{x}(\mathrm{BCG})<100 \mathrm{kpc}$. Even when $r_{x}(\mathrm{BCG})>100$ $\mathrm{kpc}$, there are only a handful of M2s with $r_{x}(\mathrm{M} 2)<50$ kpc; of these there are only three M2s with luminosities within $0.3 \mathrm{mag}$ of their BCGs. In short, even though we have searched for and selected BCGs at large distances from the X-ray centers of their clusters, we are not overlooking a large population of bright "central" galaxies that might plausibly be better choices for the dominant galaxy in the cluster.

There are other ways in which the population of M2 galaxies differs from that of the BCGs besides just being less luminous. Figure 28 compares the $L_{m}-\alpha$ properties of the two sets. In this case, we plot $M_{m}$ as the independent variable, instead of assigning this role to $\alpha$ as we did in 84.2 . Intriguingly, this figure echoes the conclusion suggested by Figures 25 and 26 that the differences between M2 and the BCGs are most important for the brightest BCGs. For $M_{m}>-22.75$, the M2 and BCG galaxies have essentially indistinguishable distributions of $\alpha$ as a function of $M_{m}$. It's also worth noting that given the large scatter in the $L_{m}-\alpha$ relation, many of the M2s may actually have larger $\alpha$ values than do their corresponding BCGs. For $M_{m}<-23$, however, while the mean $\alpha$ continues to increase for BCGs, it does not for the M2s. The few M2s with $M_{m}<-23$ have significantly lower $\alpha$ on average than the corresponding set of BCGs. Considering the results from both the previous and following sections, this implies that the bright M2s are galaxies that are not likely to be close to the X-ray center of their clusters. Bright M2s with more "normal" $\alpha$ would be more centrally located, and thus vulnerable to merging with the BCG. The median $r_{x}$ for M2 with $M_{m} \leq-22.5$ is $327 \mathrm{kpc}$, indeed showing that the brightest $\mathrm{M} 2 \mathrm{~s}$ are most likely to be found markedly displaced from the center of the cluster.

Additional insight into the differences between M2 and the BCG comes from considering their relative velocity and spatial displacements within their clusters. Figure 29 plots luminosity difference between M2 and the BCG, 


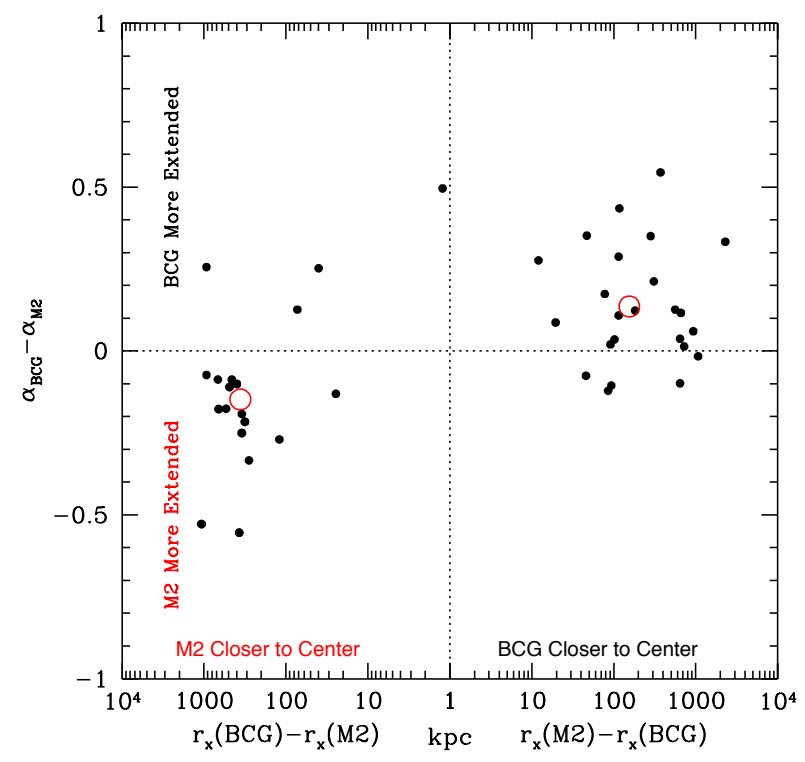

Figure 32. The difference in $\alpha$ between the BCG and M2 in a given cluster is plotted as a function of the difference between their radial offsets from the X-ray center in clusters where $M_{m}(\mathrm{M} 2)-$ $M_{m}(\mathrm{BCG})<0.3 \mathrm{mag}$. The left half of the graph plots clusters in which the BCG is further from the X-ray center than the M2 galaxy, while the right side plots clusters in which M2 is further away. The red circle indicates the mean $\Delta \alpha$ for each half of the figure. When M2 and the BCG are close in luminosity the galaxy closest to the X-ray center is most likely to have the larger $\alpha$.

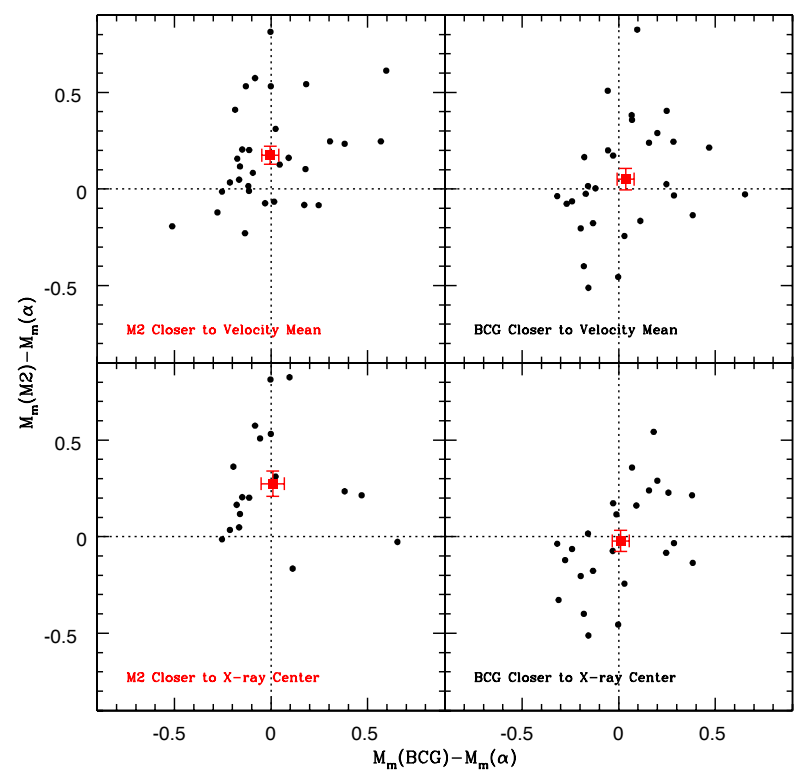

Figure 33. The luminosity residuals of the BCGs from the $L_{m}-\alpha$ relation (equation 8) are plotted against those of M2 for clusters in which $M_{m}(\mathrm{M} 2)-M_{m}(\mathrm{BCG})<0.3 \mathrm{mag}$. This set of clusters is further divided into four subsets depending on whether the BCG or M2 is closer to the mean cluster velocity or X-ray center. The red symbol in each panel shows the mean BCG and M2 luminosity residuals. When M2 is further from the velocity or X-ray center it appears to follow the $L_{m}-\alpha$ relation. M2s closer to the center, however, are significantly dimmer than predicted by the $L_{m}-\alpha$ relation, given their large $\alpha$ values but lower metric luminosities.

$M_{m}(\mathrm{M} 2)-M_{m}(\mathrm{BCG})$ as a function of either the peculiar velocity of the BCG with the cluster (left panel) or that of M2 (right panel) for clusters with 25 or more members (so as to minimize the contribution of the error in the mean redshift to the peculiar velocities). It is striking that $M_{m}(\mathrm{M} 2)-M_{m}(\mathrm{BCG})<0.5 \mathrm{mag}$ for nearly all clusters in which the BCG $\Delta V_{1}>400 \mathrm{~km} \mathrm{~s}^{-1}$. This suggests a picture in which the BCGs with high peculiar velocities are relatively recent additions to the cluster that have not had enough time to undergo the relaxation interactions that would move them closer to the cluster velocity centroid. In this case the M2 is likely to be the "former BCG," reflecting its rank prior to the infall of a more luminous galaxy. However, a fraction of these M2s will ultimately merge with the "new" BCG once the latter's peculiar velocity has been reduced enough to make merging interactions possible. The "new" M2 will be less luminous than the old M2 that merged with the $\mathrm{BCG}$, thus increasing the BCG-M2 luminosity difference for that cluster.

The effect of the BCG on M2 is also visible when $M_{m}(\mathrm{M} 2)-M_{m}(\mathrm{BCG})$ is plotted as function of the BCG $\mathrm{X}$-ray offset (Figure 30). The main signature is that there are no M2s with $M_{m}(\mathrm{M} 2)-M_{m}(\mathrm{BCG})<0.2 \mathrm{mag}$ once the BCG gets within $20 \mathrm{kpc}$ of the X-ray center. Conversely, there appears to be no relationship between $M_{m}$ (M2) $-M_{m}$ (BCG) and $r_{x}$ for BCGs falling outside this radius. The figure also ratifies the conclusion discussed in the context of Figure 27 that there are essentially no M2s closer to the center than the BCG once $r_{x}(\mathrm{BCG})<100 \mathrm{kpc}$.

The structure of M2 also appears to be affected by its proximity to the mean velocity of its hosting cluster, as is the case for the BCGs. Figure 31 compares $\alpha$ between the BCG and M2 in clusters (with 25 or more galaxy velocities) in which $\mathrm{M} 2$ is a close rival of the $\mathrm{BCG}$ $\left(M_{m}(\mathrm{M} 2)-M_{m}(\mathrm{BCG})<0.3 \mathrm{mag}\right)$. On average, the $\mathrm{M} 2 \mathrm{~s}$ should have smaller $\alpha$, given their smaller $L_{m}$; however, the figure shows that this is clearly over-ridden by the effect of the proximity of the galaxies to the velocity centroid of the cluster. The M2s have slightly larger $\alpha$ when $\left|\Delta V_{2}\right|<\left|\Delta V_{1}\right|$, that is when M2 has the smaller peculiar velocity, while the BCGs nearly always have large $\alpha$ when the situation is reversed. The $\alpha$ differences are not symmetrical with the difference in absolute peculiar velocities, given the initial bias for the BCGs to have higher $\alpha$, but the effect is clear. The processes that increase $\alpha$ as the BCG approaches the center of the cluster are also in play for M2.

Likewise, when $M_{m}(\mathrm{M} 2)-M_{m}(\mathrm{BCG})<0.3 \mathrm{mag}$, the galaxy of the two that is closest to the $\mathrm{X}$-ray center of the cluster is more likely to have the higher $\alpha$. Figure 32 plots the difference in $\alpha$ between the BCG and M2 as function of the difference between each galaxy's offset from the X-ray center. For the 24 clusters with $M_{m}(\mathrm{M} 2)-M_{m}(\mathrm{BCG})<0.3$ (and X-ray positions available) and the BCG closer to the X-ray center than M2 (right half of the figure), $\alpha(\mathrm{M} 2)-\alpha(\mathrm{BCG})=-0.14 \pm 0.4$. When M2 is closer to the center (left half of the figure), the situation is reversed, with $\alpha(\mathrm{M} 2)-\alpha(\mathrm{BCG})=$ $+0.15 \pm 0.5$ for the 17 clusters in this subset.

The finding that rival M2s have larger $\alpha$ than their corresponding BCGs despite their smaller metric luminosities when closer to the X-ray or velocity centers can the BCGs implies that they will strongly deviate from 
the $L_{m}-\alpha$ relation. This is shown in Figure 33, which plots the BCG versus M2 luminosity residuals from the $L_{m}-\alpha$ relation (equation 8) for clusters in which the BCG has $M_{m}(\mathrm{M} 2)-M_{m}(\mathrm{BCG})<0.3 \mathrm{mag}$. The set of clusters is divided into four subsets depending on which of the BCG or M2 is closer to the velocity or X-ray center. When the M2 is further from either center, both the M2 and BCG luminosity residuals average to zero, showing that the M2s act as essentially lower-luminosity BCGs. Interestingly, the residuals of both galaxies appear to be correlated for this subset. When M2 is closer to either the velocity of X-ray center, however, $M_{m}(\mathrm{M} 2)$ is significantly dimmer than predicted from their large $\alpha$ values. M2s closer to the cluster center than their matching BCGs cannot be simply considered to be less luminous examples of a BCG.

\section{THE PRESENT STRUCTURE OF BCGS AS A REFLECTION OF THEIR ORIGIN}

The initial motivation of this work was to define a BCG sample to extend our earlier studies of deviations from the smooth Hubble flow (Lauer \& Postman 1992, 1994 from the $15 \mathrm{~K}$ velocity limit to $24 \mathrm{~K}$. As such, a large portion of this paper was concerned with revisiting the complete definition of the sample, including selection of the galaxy clusters, selection of the BCG, measurement of the photometry, spectroscopy, and so on. Compared to the LP94 sample, the present increase in limiting redshift leads to a substantial increase in sample size; the $15 \mathrm{~K}$ sample included 119 BCGs, while the $24 \mathrm{~K}$ sample comprises 433 galaxies. Apart from its present use to elucidate the present structure of BCGs, the sample represents a substantial, full-sky collection of precise BCG photometry, BCG central stellar velocity dispersions, cluster redshifts, and cluster velocity dispersions for nearby galaxy clusters, all of which can be used for many other investigations. We return to the questions posed in the Introduction, and finish with an attempt to integrate these results into an improved picture of the origin and evolution of BCGs.

\subsection{What Are the Properties of BCGs?}

The initial survey of BCG photometric parameters shows essentially identical results to those presented in PL95. The distribution of $M_{m}$ is Gaussian with a dispersion of 0.337 mags, and the $L_{m}-\alpha$ relation has scatter of $0.267 \mathrm{mag}$ in the CMB frame. We now also offer a form that predicts $M_{m}$ from a linear relationship in $\log \alpha$, rather than the quadratic form of PL95, due to its monotonic behavior with $\alpha$; it also provides an acceptable fit to the data. It is notable that the random scatter in $M_{m}$ has not been reduced from that presented in PL95, despite new photometry obtained for most of the $15 \mathrm{~K}$ BCGs, and improved cluster velocities.

The present work goes beyond PL95 by including central stellar velocity-dispersion observations of the BCGs. Use of metric luminosities obtained in different aperture sizes allows us to track the flattening of the Faber \& Jackson (1976) relationship between $L$ and $\sigma$ as the aperture grows to include a larger fraction of the total galaxy luminosity. The relationship between $L_{m}$ and $\sigma$ using our standard $14.3 \mathrm{kpc}$ metric radius resembles the classic $\sigma \propto L^{1 / 4}$ relation, while increasing $r_{m} 4 \times$ larger to $57.1 \mathrm{kpc}$ yields a much shallower $\sigma \propto L^{1 / 6}$ relation. The flatter Faber-Jackson relation for BCGs has been discussed extensively in the literature, but this shows that it is likely a consequence of the extremely large envelopes of the BCGs.

The residuals of the $L_{m}-\alpha$ relationship correlate strongly with $\sigma$, thus motivating the development of a three-parameter "metric plane" relationship between $L_{m}, \alpha$, and $\sigma$ analogous to the "fundamental plane" relations. Use of $\alpha$ and $\sigma$ together predicts $M_{m}$ to a precision of 0.206 mag, a substantial improvement over the $L_{m}-\alpha$ relationship. The metric plane relation also implicitly removes correlations between the residuals of the $L_{m}-\alpha$ relation and the cluster velocity dispersion.

\subsection{Where Are the BCGs Located in Their Galaxy Clusters?}

A first step in understanding the relationship of BCGs to their galaxy clusters is to ask where they are located in the clusters. In answering this question we have produced quantitative distribution functions for both the projected spatial and peculiar velocities of the BCGs with the cluster, where the spatial offset is defined with respect to the cluster X-ray center, and the velocity with respect to the mean cluster velocity. The spatial offset, $r_{x}$ follows a steep power-law with $\gamma=-2.33$ over three decades in radius; there is no evidence for any core at scales $>10 \mathrm{kpc}$.

The absolute normalized peculiar velocities $\left|\Delta V_{1}\right| / \sigma_{c}$ of BCGs within their clusters follows an exponential distribution, with scale length 0.39 . The spatial and velocity offsets are correlated. Large $\left|\Delta V_{1}\right| / \sigma_{c}$ always corresponds to large $r_{x}$ and small $r_{x}$ always corresponds to small $\left|\Delta V_{1}\right| / \sigma_{c}$.

These results raise an important caveat in understanding the relation between the BCGs and their hosting clusters. While BCGs do prefer to reside near the central regions of galaxy clusters, BCGs with $r_{x}>100 \mathrm{kpc}$ or $\left|\Delta V_{1}\right| / \sigma_{c}>0.5$ are common. These outlying BCGs further follow the same metric plane as do those closer to the center of their parent clusters. This has important consequences for understanding the relationship between "intercluster light" (ICL) and the extended envelopes of BCGs. BCGs are often simplistically assumed to always reside at both the spatial and velocity center of ICL, such that it becomes ambiguous as to where the BCG ends and the ICL picks up (see the discussion in Lauer et al. 2007). While this may be true in some cases, it will not be true in general.

Our BCGs are defined by metric luminosity, while some authors choose the brightest galaxy close to the X-ray center, even if it turns out to be the M2. The latter definition would risk the possibility of missing BCG that are still being accreted by rich galaxy clusters at the present epoch. There is no question that there are firstranked galaxies in many clusters that are offset by large velocities and projected separations for the X-ray defined centers. These are most easily understood as recently accreted additions to the cluster. The identity of the galaxy that occupies the first-ranked position will change as new galaxies are brought in, and may have changed a number of times in a given cluster over cosmological history, as is found in the simulations of De Lucia \& Blaizot (2007) and Martel et al. (2014). 


\subsection{How Does the Cluster Environment Influence the Properties of the BCGs?}

The relationships that we have observed between the properties of the BCGs and their clusters support the picture that the bulk of any given BCG is largely assembled outside of the cluster, before the galaxy is accreted by the cluster. The strongest effect is that $\alpha$ is clearly moderated by both $r_{x}$ and $\left|\Delta V_{1}\right| / \sigma_{c}$, such that $\alpha$ increases monotonically as the BCG lies closer and closer to the center of the cluster. BCGs with the largest $\alpha$ for their $L_{m}$ always reside close to the X-ray and velocity center of the cluster. Conversely, BCGs with the smallest $\alpha$ given $L_{m}$ are strongly displaced from the center, often with $r_{x} \gg 100 \mathrm{kpc}$ and $\left|\Delta V_{1}\right| / \sigma_{c}>0.5$. However, $L_{m}$ is only weakly, if at all, related to the position of the BCG within the cluster.

We conclude that the envelopes of the BCGs are expanded by and perhaps even grown by interactions that become increasingly important as the BCG spends the majority of the time in ever denser regions of the clusters and dynamical relaxation reduces its peculiar velocity. The fact that $L_{m}$ does not vary with spatial or velocity offset from the center of the cluster argues that the denser central body of the BCG, however, is less affected the same processes. Some BCGs, even at low redshifts, have been recently accreted into the outskirts of the clusters. Even though they may not have the classic extended envelopes associated with, say, massive $\mathrm{cD}$ galaxies, their $L_{m}$ is already high enough that they can claim first rank over all other galaxies within the cluster.

It is here that the properties of the M2 galaxies are important. When M2 is close in luminosity to the BCG, we see that which of the BCG or M2 has higher $\alpha$ depends on which is closer to the mean cluster velocity or the center of the cluster potential as marked by X-ray emission. Clearly, the processes that act on the BCG as a function of its location in the cluster also act on M2. In fact, the luminosity differences between the two sets of galaxies reveal the nature of the competition for first rank. While our sample of M2 is incomplete, we only see large luminosity differences between them when $\left|\Delta V_{1}\right|$ is relatively small. We hypothesize that when the BCG initially enters the cluster with a high $\left|\Delta V_{1}\right|$, the previous BCG, now demoted to $\mathrm{M} 2$, can still survive as a close rival until the BCG undergoes enough interactions to be captured into the central potential of the cluster. Conversely, when it is the M2 that has a high peculiar velocity or has an orbit that keeps it mainly in the outskirts of the cluster, it can persist as close rival in luminosity to the BCG.

\subsection{A Brief History of BCGs}

Throughout the narrative our results raise the question of the extent to which BCGs grow their luminosity inside versus outside of the their present hosting clusters. We see that there are some BCGs with large $L_{m}$, meaning that the assembly of their stellar mass is essentially complete, but that also have large $r_{x}$ and large $\left|\Delta V_{1}\right| / \sigma_{c}$, which means that they are relatively recent arrivals to their hosting clusters. In the clusters for which this is so, there may also be a centrally-located M2 that many would pick as the "true" BCG - certainly, that galaxy is more likely to have the greatly extended enve- lope that's often associated with the classical pictures of BCGs. The inference is that most of the stellar mass of any given $\mathrm{BCG}$, or at least its portion within the metric radius, grew from the merger of progenitor galaxies outside the rich cluster environment. Such a scenario was suggested by Merritt (1985), who noted that the lower velocity dispersions of galaxy groups, rather than rich clusters, made them attractive as the birthplace of future BCGs. The BCG may represent the merging terminus of many of the galaxies within the group. The merged system may later be accreted by a rich cluster, but then may be subjected to only minor interactions or mergers once it arrives there. These would add energy to its envelope but little stellar mass.

On the other hand, in this study we see that BCG $L_{m}$ increases markedly with $\sigma_{c}$, and as we noted earlier in the Introduction, BCG luminosity also tracks cluster X-ray luminosity and temperature. One might presume that this only reflects the likelihood that today's rich clusters once had the richest retinue of surrounding groups, but there is evidence that major mergers can take place within clusters (Lauer 1988). The most luminous BCGs are unlikely to have strong M2 rivals for the position of first rank. Faint M2 galaxies are further uniquely associated with BCGs with modest $\left|\Delta V_{1}\right| / \sigma_{c}$. Whatever level of minor merging that may have taken place, this result suggests that many of the current BCGs have cannibalized their closest rivals, an event that would have been a major merger. It is also a feature of dry merging that this may cause little luminosity growth within the metric aperture (Hausman \& Ostriker 1978, Boylan-Kolchin et al. 2006), while greatly extending the envelope. The growth in $\alpha$ as the BCGs become more centrally located argue that this is happening.

The question of whether most BCG luminosity growth occurs inside or outside of rich clusters intersects with the issue of whether or not BCGs are "special." Lin et al. (2010) have argued that only the BCGs in the most X-ray luminous clusters exhibit luminosities high enough such that they cannot be explained as simply being drawn from a standard cluster luminosity function. This is thus a partial contradiction of the analysis of Tremaine \& Richstone (1977) that suggested that all BCGs were created by special processes. In this work we see that the M2s are most structurally similar to their corresponding BCGs when the BCGs have more modest metric luminosities. It is when $M_{m}$ starts to grow beyond $\sim-22.5$ that we begin to see their properties diverge. We conclude that if BCGs were born in relatively small groups, it is their accretion into rich clusters that later shapes and grows them to their final form and special luminosities. We will explore these themes further in follow-on papers that will elucidate the relation of the present sample of BCGs to the properties of giant elliptical galaxies and measure the on-going rate of merger interactions in BCGs.

This work is based on extensive observational work conducted at Kitt Peak National Observatory and Cerro Tololo Inter-American Observatory, both operated by the National Optical Astronomy Observatory (NOAO). We are indebted to the professional staff of NOAO for the development and operation of the telescopes and instru- 
ments that we used for this project. NOAO is operated by AURA, Inc., under cooperative agreement with the National Science Foundation. We thank Beth Reid, Megan Donahue, Kayhan Gültekin, Christine Jones, and William Forman for useful conversations. We thank Katelyn Millette for a critical reading of the manuscript. This research has made use of the NASA/IPAC Extragalactic Database (NED), which is operated by the Jet Propulsion Laboratory, California Institute of Technology, under contract with the National Aeronautics and Space Administration. This paper has also made use of the data from the SDSS. Funding for the SDSS and SDSS-II has been provided by the Alfred P. Sloan Foundation, the Participating Institutions, the National Science Foundation, the U.S. Department of Energy, the National Aeronautics and Space Administration, the Japanese Monbukagakusho, the Max Planck Society, and the Higher Education Funding Council for England.

\section{REFERENCES}

Abazajian, K. N., Adelman-McCarthy, J. K., Agüeros, M. A., et al. 2009, ApJS, 182, 543

Abell, G. O. 1958, ApJS, 3, 211

Abell, G. O., Corwin, H. G., Jr., \& Olowin, R. P. 1989, ApJS, 70,

Adelman-McCarthy, J. K., Agüeros, M. A., Allam, S. S., et al. 2008, ApJS, 175, 297

Allen, S. W. 1998, MNRAS, 296, 392

Ascaso, B., Aguerri, J. A. L., Varela, J., et al. 2011, ApJ, 726, 69

Bahcall, N., \& Soniera, R. M. 1983, ApJ, 270, 20

Bechtold, J., Forman, W., Jones, C., et al. 1983, ApJ, 265, 26

Beers, T. C., \& Geller, M. J. 1983, ApJ, 274, 491

Beers, T. C., Flynn, K., \& Gebhardt, K. 1990, AJ, 100, 32

Bernardi, M., Hyde, J. B., Sheth, R. K., Miller, C. J., \& Nichol, R. C. 2007, AJ, 133, 1741

Binggeli, B. 1982, A\&A, 107, 338

Bolton, A. S., Schlegel, D. J., Aubourg, É., et al. 2012, AJ, 144, 144

Boylan-Kolchin, M., Ma, C.-P., \& Quataert, E. 2006, MNRAS, 369, 1081

Brough, S., Collins, C. A., Burke, D. J., Lynam, P. D., \& Mann, R. G. 2005, MNRAS, 364, 1354

Burstein, D., \& Heiles, C. 1982, AJ, 87, 1165

Cappellari, M., \& Emsellem, E. 2004, PASP, 116, 138

Carlberg, R. G., Yee, H. K. C., \& Ellingson, E. 1997, ApJ, 478, 462

Cavagnolo, K. W., Donahue, M., Voit, G. M., \& Sun, M. 2009, ApJS, 182, 12

Chisari, N. E., Lauer, T. R., Postman, M., Strauss, M. A., \& Graves, G. J. 2014, in preparation.

Collins, C. A., \& Mann, R. G. 1998, MNRAS, 297, 128

Coziol, R., Andernach, H., Caretta, C. A., Alamo-Martínez, K. A., \& Tago, E. 2009, AJ, 137, 4795

De Lucia, G., \& Blaizot, J. 2007, MNRAS, 375, 2

Diaferio, A., \& Geller, M. J. 1996, ApJ, 467, 19

Djorgovski, S., \& Davis, M. 1987, ApJ, 313, 59

Dressler, A., Lynden-Bell, D., Burstein, D., et al. 1987, ApJ, 313, 42

Ebeling, H., Voges, W., Bohringer, H., et al. 1996, MNRAS, 281, 799

Edge, A. C. 1991, MNRAS, 250, 103

Edge, A. C., \& Stewart, G. C. 1991, MNRAS, 252, 414

Faber, S. M., \& Jackson, R. E. 1976, ApJ, 204, 668

Finkbeiner, D. P., Davis, M., \& Schlegel, D. J. 1999, ApJ, 524, 867

Fisher, K. B., Davis, M., Strauss, M. A., Yahil, A., \& Huchra, J. P. 1994, MNRAS, 267, 927

Geller, M. J., \& Postman, M. 1983, ApJ, 274, 31.

Graves, G. J., Faber, S. M., Schiavon, R. P., \& Yan, R. 2007, ApJ, 671, 243
Greene, J. E., Murphy, J. D., Graves, G. J., Gunn, J. E., Raskutti, S., Comerford, J. M., \& Gebhardt, K. 2013, ApJ, 776,64

Gunn, J. E., \& Oke, J. B. 1975, ApJ, 195, 255

Haarsma, D. B., Leisman, L., Donahue, M., et al. 2010, ApJ, 713, 1037

Hao, J., Kubo, J. M., Feldmann, R., et al. 2011, ApJ, 740, 39

Hashimoto, Y., Henry, J. P., \& Boehringer, H. 2014, MNRAS, 440,588

Hausman, M. A., \& Ostriker, J. P. 1978, ApJ, 224, 320

Hogg, D. W., Bovy, J., \& Lang, D. 2010, arXiv:1008.4686

Hudson, D. S., Mittal, R., Reiprich, T. H., et al. 2010, A\&A, 513, A37

Hudson, M. J., \& Ebeling, H. 1997, ApJ, 479, 621

Humason, M. L., Mayall, N. U., \& Sandage, A. R. 1956, AJ, 61, 97

Hoessel, J. G. 1980, ApJ, 241, 493

Hopkins, P. F., Bundy, K., Murray, N., et al. 2009, MNRAS, 398, 898

Jones, C., \& Forman, W. 1984, ApJ, 276, 38

Juszkiewicz, R., Fisher, K. B., \& Szapudi, I. 1998, ApJ, 504, L1

Kelly, B. C. 2011, arXiv:1112.1745

Lambas, D. G., Groth, E. J., \& Peebles, P. J. E. 1988, AJ, 95, 996

Landolt, A. U. 1983, AJ, 88, 439

Lauer, T. R. 1986, ApJ, 311, 34

Lauer, T. R. 1988, ApJ, 325, 49

Lauer, T. R., Faber, S. M., Richstone, D., et al. 2007, ApJ, 662, 808

Lauer, T. R., \& Postman, M. 1992, ApJ, 400, L47

Lauer, T. R., \& Postman, M. 1994, ApJ, 425, 418

Ledlow, M. J., Voges, W., Owen, F. N., \& Burns, J. O. 2003, AJ, 126,2740

Lin, Y.-T., Ostriker, J. P., \& Miller, C. J. 2010, ApJ, 715, 1486

Liu, F. S., Xia, X. Y., Mao, S., Wu, H., \& Deng, Z. G. 2008, MNRAS, 385, 23

Loh, Y.-S., \& Strauss, M. A. 2006, MNRAS, 366, 373

Mahdavi, A., \& Geller, M. J. 2001, ApJ, 554, L129

Malumuth, E. M. 1992, ApJ, 386, 420

Martel, H., Robichaud, F., \& Barai, P. 2014, ApJ, 786, 79

Marzke, R. O., Geller, M. J., da Costa, L. N., \& Huchra, J. P. 1995, AJ, 110, 477

Merritt, D. 1985, ApJ, 289, 18

Miller, C. J., Nichol, R. C., Reichart, D., et al. 2005, AJ, 130, 968

Newman, A. B., Treu, T., Ellis, R. S., \& Sand, D. J. 2011, ApJ, 728, L39

Niederste-Ostholt, M., Strauss, M. A., Dong, F., Koester, B. P., \& McKay, T. A. 2010, MNRAS, 405, 2023

Oegerle, W. R., \& Hill, J. M. 2001, AJ, 122, 2858

Oegerle, W. R., \& Hoessel, J. G. 1991, ApJ, 375, 15

Ostriker, J. P., \& Tremaine, S. D. 1975, ApJ, 202, L113

Patel, P., Maddox, S., Pearce, F. R., Aragón-Salamanca, A., \& Conway, E. 2006, MNRAS, 370, 851

Postman, M., Huchra, J. P., \& Geller, M. J. 1992, ApJ, 384, 404

Postman, M., \& Lauer, T. R. 1995, ApJ, 440, 28

Postman, M., Lauer, T. R., Oegerle, W., \& Donahue, M. 2002, ApJ, 579, 93

Quintana, H., \& Lawrie, D. G. 1982, AJ, 87, 1

Quintana, H., \& Melnick, J. 1982, AJ, 87, 972

Reid, B. A., Seo, H.-J., Leauthaud, A., Tinker, J. L., \& White, M. 2014, arXiv:1404.3742

Sánchez-Blázquez, P., Gorgas, J., Cardiel, N., \& González, J. J. 2006, A\&A, 457, 809

Richstone, D. O. 1975, ApJ, 200, 535

Sandage, A. 1972a, ApJ, 173, 485

Sandage, A. 1972b, ApJ, 178, 1

Sandage, A. 1975, ApJ, 202, 563

Sandage, A. 1976, ApJ, 205, 6

Sandage, A., \& Hardy, E. 1973, ApJ, 183, 743

Sastry, G. N. 1968, PASP, 80, 252

Schechter, P. 1976, ApJ, 203, 297

Schlegel, D. J., Finkbeiner, D. P., \& Davis, M. 1998, ApJ, 500, 525

Schombert, J. M. 1988, ApJ, 328, 475

Skibba, R. A., van den Bosch, F. C., Yang, X., et al. 2011, MNRAS, 410, 417

Smith, G. P., Khosroshahi, H. G., Dariush, A., et al. 2010, MNRAS, 409, 169 
Smith, G. P., Kneib, J.-P., Smail, I., et al. 2005, MNRAS, 359, 417

Solinger, A. B., \& Tucker, W. H. 1972, ApJ, 175, L107

Stephens, M. 1974, J. Am. Stat. Assoc., 69, 730

Stott, J. P., Hickox, R. C., Edge, A. C., et al. 2012, MNRAS, 422, 2213

Tonry, J., \& Davis, M. 1979, AJ, 84, 1511

Tremaine, S. D., \& Richstone, D. O. 1977, ApJ, 212, 311

van Dokkum, P. G., Whitaker, K. E., Brammer, G., et al. 2010, ApJ, 709, 1018

Vazdekis, A., Sánchez-Blázquez, P., Falcón-Barroso, J., Cenarro, A. J., Beasley, M. A., Cardiel, N., Gorgas, J., \& Peletier, R. F. 2010, MNRAS, 404, 1639
Voges, W., Aschenbach, B., Boller, T., et al. 1999, A\&A, 349, 389 von der Linden, A., Best, P. N., Kauffmann, G., \& White,

S. D. M. 2007, MNRAS, 379, 867

White, S. D. M., Briel, U. G., \& Henry, J. P. 1993, MNRAS, 261, L8

Wu, X.-P., Xue, Y.-J., \& Fang, L.-Z. 1999, ApJ, 524, 22

Yahil, A., \& Vidal, N. V. 1977, Ap.J, 214, 347

Zabludoff, A. I., Geller, M. J., Huchra, J. P., \& Ramella, M. 1993, AJ, 106, 1301

Zabludoff, A. I., Huchra, J. P., \& Geller, M. J. 1990, ApJS, 74, 1

Table 1

The BCG and Abell Cluster Sample

\begin{tabular}{|c|c|c|c|c|c|c|c|c|}
\hline Abell & $\begin{array}{c}\alpha \\
(2000)\end{array}$ & $\begin{array}{c}\delta \\
(2000)\end{array}$ & $\begin{array}{c}V_{1} \\
\mathrm{~km} \mathrm{~s}^{-1}\end{array}$ & $\begin{array}{c}V_{c} \\
\mathrm{~km} \mathrm{~s}^{-1}\end{array}$ & $\begin{array}{c}\sigma_{c} \\
\mathrm{~km} \mathrm{~s}^{-1}\end{array}$ & $N_{g}$ & $A_{B}$ & Notes \\
\hline 14 & 3.7957 & -23.8823 & $19280 \pm 26$ & $19397 \pm 111$ & $474 \pm 118$ & 18 & 0.094 & E473-G005 \\
\hline 27 & 6.1965 & -20.7324 & $15519 \pm 0$ & $16037 \pm 90$ & $339 \pm 79$ & 14 & 0.094 & E539-G013 \\
\hline 71 & 9.4947 & +29.6035 & $22044 \pm 33$ & $22044 \pm 33$ & & 2 & 0.210 & \\
\hline 74 & 9.5903 & -22.3449 & $19013 \pm 46$ & $19326 \pm 56$ & $212 \pm 59$ & 14 & 0.091 & \\
\hline 75 & 9.8692 & +21.2295 & $17610 \pm 42$ & $17355 \pm 326$ & $731 \pm 511$ & 5 & 0.146 & \\
\hline 76 & 9.8597 & +6.7340 & $11283 \pm 17$ & $11969 \pm 148$ & $491 \pm 120$ & 11 & 0.179 & I1565 15K \\
\hline 77 & 10.1181 & +29.5558 & $21424 \pm 35$ & $21135 \pm 187$ & $419 \pm 246$ & 5 & 0.190 & U00428 \\
\hline 85 & 10.4602 & -9.3034 & $16581 \pm 56$ & $16622 \pm 62$ & $1009 \pm 31$ & 263 & 0.162 & \\
\hline 86 & 10.6762 & -21.7745 & $18481 \pm 41$ & $18887 \pm 111$ & $415 \pm 72$ & 14 & 0.086 & \\
\hline 102 & 12.1301 & +1.3547 & $18946 \pm 37$ & $19702 \pm 166$ & $624 \pm 125$ & 14 & 0.124 & U00496 \\
\hline 114 & 13.5312 & -21.7143 & $17290 \pm 45$ & $17705 \pm 105$ & $515 \pm 77$ & 24 & 0.079 & \\
\hline 116 & 13.9435 & +0.6450 & $20100 \pm 42$ & $19992 \pm 92$ & $629 \pm 80$ & 46 & 0.122 & \\
\hline 117 & 13.9662 & -9.9857 & $16453 \pm 47$ & $16460 \pm 63$ & $629 \pm 49$ & 99 & 0.156 & I1602 \\
\hline 119 & 14.0672 & -1.2561 & $13338 \pm 22$ & $13334 \pm 55$ & $901 \pm 40$ & 267 & 0.167 & U00579 $15 \mathrm{~K}$ \\
\hline 133 & 15.6739 & -21.8820 & $17041 \pm 50$ & $16759 \pm 94$ & $790 \pm 63$ & 70 & 0.080 & E541-G013 \\
\hline 134 & 15.6628 & -2.6962 & $20639 \pm 31$ & $21000 \pm 86$ & $345 \pm 61$ & 16 & 0.190 & \\
\hline 147 & 17.1579 & +2.2684 & $13149 \pm 56$ & $13030 \pm 115$ & $621 \pm 79$ & 29 & 0.108 & $15 \mathrm{~K}$ \\
\hline 150 & 17.3268 & +13.1687 & $17754 \pm 38$ & $17750 \pm 191$ & $664 \pm 151$ & 12 & 0.122 & U00716 \\
\hline 151 & 17.2130 & -15.4072 & $15936 \pm 49$ & $16011 \pm 66$ & $795 \pm 59$ & 144 & 0.113 & I0080 \\
\hline 152 & 17.5135 & +13.9778 & $17491 \pm 35$ & $17611 \pm 99$ & $844 \pm 59$ & 72 & 0.154 & U00727 \\
\hline 154 & 17.7647 & +17.6518 & $18236 \pm 39$ & $19115 \pm 183$ & $988 \pm 146$ & 29 & 0.276 & I1635 \\
\hline 158 & 17.9387 & +16.8862 & $18958 \pm 38$ & $18958 \pm 38$ & $\ldots$ & 2 & 0.349 & \\
\hline 161 & 18.8433 & $\begin{array}{l}+37.3396 \\
\end{array}$ & $22185 \pm 45$ & $22185 \pm 45$ & & 2 & 0.279 & \\
\hline 168 & 18.7404 & +0.4311 & $13546 \pm 50$ & $13476 \pm 54$ & $625 \pm 36$ & $13 \overline{3}$ & 0.149 & U00797 15K \\
\hline 171 & 19.3248 & +16.2652 & $20718 \pm 45$ & $20718 \pm 45$ & & 2 & 0.466 & \\
\hline 174 & 20.0677 & +35.8072 & $22490 \pm 43$ & $22490 \pm 43$ & & 2 & 0.250 & \\
\hline 179 & 20.5452 & +19.6205 & $15618 \pm 39$ & $16187 \pm 127$ & $284 \pm 0$ & 5 & 0.224 & \\
\hline 189 & 20.8597 & $\begin{array}{r}1 \\
+1.7049\end{array}$ & $10195 \pm 14$ & $9600 \pm 66$ & $332 \pm 46$ & 25 & 0.142 & VV $50915 \mathrm{~K}$ \\
\hline 193 & 21.2814 & +8.6992 & $14664 \pm 43$ & $14529 \pm 78$ & $776 \pm 62$ & 97 & 0.219 & I1695 15K \\
\hline 194 & 21.4952 & -1.3394 & $5296 \pm 20$ & $5390 \pm 43$ & $495 \pm 29$ & 132 & 0.182 & N0545 15K \\
\hline 195 & 21.7281 & +19.2139 & $12915 \pm 60$ & $12656 \pm 85$ & $306 \pm 58$ & 13 & 0.236 & $\mathrm{I} 011515 \mathrm{~K}$ \\
\hline 208 & 22.8873 & +0.5558 & $23802 \pm 41$ & $23729 \pm 76$ & $601 \pm 50$ & 61 & 0.102 & \\
\hline 225 & 24.7043 & +18.8251 & $20819 \pm 41$ & $20832 \pm 269$ & $660 \pm 272$ & 6 & 0.249 & \\
\hline 240 & 25.5252 & +7.6646 & $18253 \pm 36$ & $18253 \pm 36$ & & 3 & 0.181 & U01191 \\
\hline 245 & 26.0341 & +6.3162 & $23391 \pm 34$ & $23391 \pm 34$ & & 2 & 0.240 & \\
\hline 246 & 26.1812 & $\begin{array}{r}+5.8119 \\
\end{array}$ & $22691 \pm 51$ & $22617 \pm 158$ & $571 \pm 138$ & 13 & 0.308 & \\
\hline 257 & 27.2851 & +13.9629 & $21143 \pm 42$ & $21054 \pm 72$ & $499 \pm 42$ & 47 & 0.239 & \\
\hline 260 & 27.6790 & +33.0819 & $10642 \pm 19$ & $10931 \pm 90$ & $754 \pm 74$ & 70 & 0.210 & I1733 15K \\
\hline 261 & 27.8603 & -2.2532 & $13965 \pm 21$ & $14159 \pm 69$ & $183 \pm 61$ & 7 & 0.125 & $15 \mathrm{~K}$ \\
\hline 262 & 28.1928 & +36.1515 & $4813 \pm 41$ & $4860 \pm 44$ & $540 \pm 38$ & 150 & 0.373 & N0708 15K \\
\hline 267 & 28.3134 & +1.0388 & $17963 \pm 34$ & $17878 \pm 46$ & $296 \pm 42$ & 40 & 0.108 & \\
\hline 268 & 28.2052 & -1.0018 & $17917 \pm 29$ & $17925 \pm 35$ & $160 \pm 88$ & 20 & 0.121 & \\
\hline 279 & 29.0707 & +1.0507 & $23680 \pm 34$ & $24013 \pm 78$ & $753 \pm 78$ & 92 & 0.126 & \\
\hline 292 & 30.5789 & +19.0669 & $19423 \pm 48$ & $19423 \pm 48$ & & 3 & 0.340 & U01518 \\
\hline 295 & 30.5718 & -1.1279 & $12813 \pm 56$ & $12694 \pm 49$ & $406 \pm 37$ & 66 & 0.122 & U01525 15K \\
\hline 311 & 32.3687 & +19.7761 & $19580 \pm 43$ & $19580 \pm 43$ & & 1 & 0.751 & \\
\hline 326 & 33.4156 & -7.1453 & $17052 \pm 51$ & $16503 \pm 216$ & $483 \pm 0$ & 5 & 0.126 & \\
\hline 347 & 36.3603 & +41.8242 & $5234 \pm 14$ & $5628 \pm 80$ & $627 \pm 61$ & 60 & 0.251 & N0910 15K \\
\hline 357 & 37.3652 & $\begin{array}{r}+13.2661 \\
\end{array}$ & $16948 \pm 48$ & $16948 \pm 48$ & & 3 & 0.461 & \\
\hline 358 & 37.4957 & -13.2649 & $16951 \pm 41$ & $16855 \pm 97$ & $322 \pm 232$ & 11 & 0.073 & \\
\hline 376 & 41.5168 & +36.9046 & $14560 \pm 36$ & $14394 \pm 72$ & $830 \pm 59$ & 130 & 0.314 & U02232 15K \\
\hline 386 & 42.5095 & -17.0484 & $17835 \pm 34$ & $17835 \pm 34$ & & 3 & 0.112 & \\
\hline 397 & 44.1205 & +15.9164 & $10381 \pm 17$ & $9920 \pm 111$ & $638 \pm 85$ & 33 & 0.650 & U02413 15K \\
\hline 399 & 44.4715 & +13.0305 & $21415 \pm 49$ & $21550 \pm 110$ & $1224 \pm 62$ & 122 & 0.759 & U02438 \\
\hline 400 & 44.4240 & $\begin{array}{l}+6.0214 \\
\end{array}$ & $7250 \pm 10$ & $7281 \pm 63$ & $683 \pm 39$ & 116 & 0.779 & N1128 \\
\hline 401 & 44.7410 & +13.5826 & $22215 \pm 58$ & $22045 \pm 104$ & $1161 \pm 76$ & 124 & 0.678 & U02450 \\
\hline 404 & 45.3950 & $\begin{array}{l}+41.4873 \\
\end{array}$ & $18420 \pm 34$ & $18686 \pm 101$ & $202 \pm 145$ & 4 & 0.525 & \\
\hline 407 & 45.4650 & $\begin{array}{r}+35.8409 \\
\end{array}$ & $14059 \pm 110$ & $13872 \pm 85$ & $762 \pm 62$ & 80 & 0.843 & U02489 15K \\
\hline 415 & 46.7200 & -12.1063 & $23588 \pm 53$ & $24025 \pm 192$ & $665 \pm 130$ & 12 & 0.257 & \\
\hline 423 & 47.8220 & -12.1284 & $23435 \pm 49$ & $24147 \pm 404$ & $1280 \pm 315$ & 10 & 0.271 & \\
\hline
\end{tabular}


Table 1 - Continued

\begin{tabular}{|c|c|c|c|c|c|c|c|c|}
\hline Abell & $\begin{array}{c}\alpha \\
(2000)\end{array}$ & $\begin{array}{c}\delta \\
(2000)\end{array}$ & $\begin{array}{c}V_{1} \\
\mathrm{~km} \mathrm{~s}^{-1}\end{array}$ & $\begin{array}{c}V_{c} \\
\mathrm{~km} \mathrm{~s}^{-1}\end{array}$ & $\begin{array}{c}\sigma_{c} \\
\mathrm{~km} \mathrm{~s}^{-1}\end{array}$ & $N_{g}$ & $A_{B}$ & Notes \\
\hline 428 & 49.0522 & -19.0822 & $19908 \pm 42$ & $20024 \pm 62$ & $317 \pm 51$ & 26 & 0.127 & \\
\hline 436 & 51.6473 & +9.1841 & $19061 \pm 51$ & $19061 \pm 51$ & 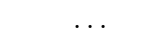 & 2 & 1.189 & \\
\hline 450 & 55.2308 & +23.5922 & $17685 \pm 51$ & $17685 \pm 51$ & & 2 & 0.881 & \\
\hline 496 & 68.4070 & -13.2610 & $9839 \pm 7$ & $9899 \pm 42$ & $737 \pm 29$ & 305 & 0.586 & $15 \mathrm{~K}$ \\
\hline 498 & 69.4616 & +21.2053 & $17399 \pm 55$ & $17399 \pm 55$ & & 1 & 1.725 & \\
\hline 500 & 69.7190 & -22.1110 & $20340 \pm 48$ & $20347 \pm 80$ & $771 \pm 175$ & 92 & 0.215 & \\
\hline 505 & 74.9836 & +80.1784 & $16017 \pm 47$ & $16017 \pm 47$ & & 1 & 0.409 & U03197 \\
\hline 514 & 71.7771 & -20.4811 & $21359 \pm 49$ & $21638 \pm 129$ & $1180 \pm 69$ & 83 & 0.237 & \\
\hline 533 & 75.2844 & -22.5828 & $14384 \pm 28$ & $14563 \pm 119$ & $610 \pm 157$ & 26 & 0.151 & $15 \mathrm{~K}$ \\
\hline 539 & 79.2297 & +6.5526 & $9690 \pm 23$ & $8710 \pm 94$ & $833 \pm 40$ & 78 & 0.726 & $15 \mathrm{~K}$ \\
\hline 548 & 87.3406 & -25.3464 & $11858 \pm 19$ & $12293 \pm 59$ & $795 \pm 28$ & 178 & 0.124 & E488-G033 15K \\
\hline 564 & 105.1685 & +69.9069 & $23188 \pm 34$ & $23188 \pm 34$ & $0 \pm 70$ & 3 & 0.243 & \\
\hline 568 & 106.9232 & +35.0582 & $23496 \pm 48$ & $23025 \pm 259$ & $687 \pm 0$ & 7 & 0.277 & \\
\hline 569 & 107.2823 & +48.6154 & $5839 \pm 3$ & $5949 \pm 58$ & $394 \pm 25$ & 45 & 0.307 & N2329 15K \\
\hline 576 & 110.5285 & +55.8748 & $12087 \pm 21$ & $11503 \pm 76$ & $1093 \pm 37$ & 205 & 0.325 & $15 \mathrm{~K}$ \\
\hline 582 & 112.0035 & +41.9185 & $17518 \pm 5$ & $17336 \pm 74$ & $324 \pm 56$ & 19 & 0.375 & \\
\hline 592 & 115.7208 & +9.2809 & $18697 \pm 35$ & $18846 \pm 55$ & $123 \pm 0$ & 5 & 0.154 & \\
\hline 595 & 117.1535 & +52.2220 & $19282 \pm 36$ & $20705 \pm 85$ & $601 \pm 56$ & 49 & 0.237 & \\
\hline 600 & 119.1492 & +63.7397 & $23302 \pm 34$ & $23302 \pm 34$ & & 3 & 0.216 & \\
\hline 602 & 118.1166 & +29.5423 & $17784 \pm 28$ & $18137 \pm 93$ & $796 \pm 61$ & 73 & 0.244 & \\
\hline 634 & 123.9371 & +58.3211 & $8139 \pm 13$ & $7921 \pm 38$ & $331 \pm 25$ & 74 & 0.238 & U04289 15K \\
\hline 644 & 124.3569 & -7.5128 & $21176 \pm 43$ & $20982 \pm 148$ & $960 \pm 114$ & 42 & 0.471 & \\
\hline 671 & 127.1330 & +30.4300 & $14970 \pm 120$ & $14877 \pm 75$ & $850 \pm 33$ & 126 & 0.203 & $\mathrm{I} 237815 \mathrm{~K}$ \\
\hline 690 & 129.8163 & +28.8439 & $23750 \pm 250$ & $24045 \pm 66$ & $546 \pm 46$ & 68 & 0.240 & \\
\hline 695 & 130.3047 & +32.4161 & $20560 \pm 84$ & $20282 \pm 80$ & $402 \pm 52$ & 25 & 0.168 & \\
\hline 744 & 136.8357 & +16.6514 & $21865 \pm 25$ & $21844 \pm 76$ & $445 \pm 920$ & 34 & 0.150 & \\
\hline 754 & 137.1347 & -9.6303 & $16440 \pm 57$ & $16374 \pm 50$ & $995 \pm 35$ & 389 & 0.277 & \\
\hline 757 & 138.2826 & +47.7082 & $15415 \pm 0$ & $15410 \pm 46$ & $360 \pm 32$ & 61 & 0.072 & \\
\hline 779 & 139.9450 & +33.7496 & $6897 \pm 1$ & $6885 \pm 39$ & $450 \pm 23$ & 133 & 0.073 & N2832 15K \\
\hline 780 & 139.5238 & -12.0955 & $16286 \pm 8$ & $16356 \pm 139$ & $871 \pm 112$ & 39 & 0.193 & \\
\hline 819 & 143.0709 & +9.6831 & $22912 \pm 95$ & $22757 \pm 96$ & $654 \pm 66$ & 46 & 0.166 & \\
\hline 834 & 145.3872 & +66.7105 & $21307 \pm 50$ & $20910 \pm 68$ & $392 \pm 0$ & 33 & 0.602 & \\
\hline 838 & 144.2860 & -5.0417 & $15668 \pm 39$ & $15524 \pm 143$ & $477 \pm 120$ & 11 & 0.201 & \\
\hline 841 & 144.6500 & -4.3353 & $20838 \pm 34$ & $20838 \pm 34$ & & 3 & 0.229 & \\
\hline 912 & 150.2892 & -0.0796 & $13580 \pm 8$ & $13660 \pm 66$ & $369 \pm 49$ & 31 & 0.158 & $15 \mathrm{~K}$ \\
\hline 957 & 153.4095 & -0.9254 & $13387 \pm 19$ & $13497 \pm 70$ & $772 \pm 52$ & 119 & 0.181 & U05515 15K \\
\hline 970 & 154.3572 & -10.6892 & $17496 \pm 38$ & $17682 \pm 72$ & $841 \pm 75$ & 135 & 0.233 & \\
\hline 978 & 155.1107 & -6.5270 & $16211 \pm 29$ & $16215 \pm 87$ & $837 \pm 55$ & 91 & 0.181 & \\
\hline 979 & 155.0804 & -7.8937 & $15907 \pm 39$ & $15919 \pm 129$ & $484 \pm 86$ & 14 & 0.214 & \\
\hline 993 & 155.4832 & -4.8905 & $16300 \pm 52$ & $16213 \pm 50$ & $513 \pm 37$ & 104 & 0.212 & \\
\hline 999 & 155.8494 & +12.8349 & $9750 \pm 10$ & $9621 \pm 38$ & $286 \pm 25$ & 56 & 0.175 & $15 \mathrm{~K}$ \\
\hline 1003 & 156.2570 & +47.8416 & $19153 \pm 40$ & $18726 \pm 72$ & $501 \pm 50$ & 48 & 0.049 & VV 675 \\
\hline 1016 & 156.7830 & +11.0103 & $9711 \pm 5$ & $9693 \pm 30$ & $204 \pm 53$ & 45 & 0.137 & I0613 15K \\
\hline 1020 & 156.9567 & +10.4416 & $19611 \pm 10$ & $19598 \pm 68$ & $314 \pm 41$ & 21 & 0.148 & \\
\hline 1032 & 157.5425 & +4.0068 & $19823 \pm 40$ & $19990 \pm 114$ & $706 \pm 47$ & 38 & 0.141 & \\
\hline 1035 & 158.0584 & +40.2711 & $23951 \pm 150$ & $23524 \pm 115$ & $814 \pm 79$ & 50 & 0.056 & \\
\hline 1060 & 159.1773 & -27.5286 & $3858 \pm 5$ & $3714 \pm 37$ & $712 \pm 25$ & 371 & 0.342 & N3311 15K \\
\hline 1066 & 159.9119 & +5.1751 & $21216 \pm 7$ & $20718 \pm 79$ & $817 \pm 55$ & 105 & 0.110 & \\
\hline 1069 & 159.9313 & -8.6870 & $19612 \pm 57$ & $19589 \pm 85$ & $706 \pm 66$ & 69 & 0.175 & \\
\hline 1090 & 161.4225 & -18.3571 & $22994 \pm 0$ & $22994 \pm 0$ & & 1 & 0.174 & \\
\hline 1100 & 162.1905 & +22.2176 & $13981 \pm 35$ & $13947 \pm 47$ & $451 \pm 0$ & 89 & 0.093 & $15 \mathrm{~K}$ \\
\hline 1139 & 164.5459 & +1.6043 & $11515 \pm 20$ & $11839 \pm 37$ & $436 \pm 25$ & 136 & 0.135 & U06057 15K \\
\hline 1142 & 165.1891 & +10.5532 & $10138 \pm 39$ & $10595 \pm 79$ & $757 \pm 44$ & 91 & 0.125 & I0664 15K \\
\hline 1145 & 165.3681 & +16.7680 & $20704 \pm 100$ & $20565 \pm 49$ & $322 \pm 134$ & 43 & 0.082 & \\
\hline 1149 & 165.7401 & +7.6031 & $21247 \pm 35$ & $21522 \pm 50$ & $314 \pm 37$ & 39 & 0.173 & \\
\hline 1155 & 166.1649 & $\begin{array}{r}1.001 \\
+35.2295\end{array}$ & $22139 \pm 95$ & $22063 \pm 52$ & $277 \pm 41$ & 28 & 0.101 & \\
\hline 1169 & 166.9560 & +43.9166 & $17531 \pm 6$ & $17664 \pm 78$ & $738 \pm 49$ & 89 & 0.050 & \\
\hline 1171 & 166.8750 & +2.9101 & $22666 \pm 38$ & $22459 \pm 91$ & $388 \pm 56$ & 18 & 0.225 & \\
\hline 1177 & 167.4355 & +21.7588 & $9561 \pm 26$ & $9597 \pm 49$ & $331 \pm 59$ & 45 & 0.077 & N3551 15K \\
\hline 1185 & 167.6597 & +28.7675 & $10521 \pm 30$ & $9791 \pm 56$ & $758 \pm 54$ & 183 & 0.124 & N3550 15K \\
\hline 1186 & 168.5090 & $\begin{array}{r}+75.4309 \\
\end{array}$ & $23540 \pm 30$ & $23540 \pm 30$ & & 1 & 0.263 & \\
\hline 1187 & 167.7902 & +39.5976 & $23714 \pm 0$ & $22397 \pm 118$ & $952 \pm$ & 65 & 0.094 & \\
\hline 1190 & 167.9320 & +40.8205 & $23489 \pm 36$ & $22584 \pm 64$ & $671 \pm 43$ & 110 & 0.075 & \\
\hline 1203 & 168.4510 & +40.2856 & $22655 \pm 38$ & $22565 \pm 55$ & $552 \pm 36$ & 99 & 0.121 & \\
\hline 1213 & 169.0953 & +29.2523 & $13535 \pm 35$ & $14051 \pm 51$ & $572 \pm 43$ & 125 & 0.080 & $15 \mathrm{~K}$ \\
\hline 1216 & 169.5337 & -4.4303 & $15885 \pm 24$ & $15994 \pm 78$ & $390 \pm 51$ & 25 & 0.217 & \\
\hline 1228 & 170.3466 & +34.3568 & $10674 \pm 29$ & $10549 \pm 28$ & $246 \pm 23$ & 76 & 0.102 & $\mathrm{I} 273815 \mathrm{~K}$ \\
\hline 1238 & 170.7267 & $\begin{array}{r}+1.1142 \\
\end{array}$ & $21628 \pm 150$ & $22149 \pm 58$ & $564 \pm 46$ & 94 & 0.155 & \\
\hline 1257 & 171.3796 & +35.5042 & $10175 \pm 39$ & $10430 \pm 138$ & $1202 \pm 58$ & 75 & 0.098 & $15 \mathrm{~K}$ \\
\hline 1267 & 172.1517 & +26.9054 & $9795 \pm 39$ & $9880 \pm 34$ & $210 \pm 52$ & 38 & 0.081 & $15 \mathrm{~K}$ \\
\hline 1279 & 172.9149 & +67.2414 & $16258 \pm 18$ & $16293 \pm 38$ & $186 \pm 30$ & 23 & 0.050 & \\
\hline 1291 & 173.0499 & +56.0477 & $17556 \pm 67$ & $17502 \pm 78$ & $724 \pm 53$ & 85 & 0.080 & \\
\hline 1308 & 173.2712 & -4.0138 & $15535 \pm 27$ & $15465 \pm 67$ & $375 \pm 31$ & 31 & 0.199 & $15 \mathrm{~K}$ \\
\hline 1314 & 173.7059 & +49.0773 & $9999 \pm 31$ & $9877 \pm 58$ & $648 \pm 25$ & 123 & 0.071 & I0712 15K \\
\hline 1317 & 173.8044 & -13.5519 & $21632 \pm 40$ & $21706 \pm 185$ & $717 \pm 111$ & 15 & 0.160 & \\
\hline 1318 & 174.3931 & +54.8529 & $17335 \pm 37$ & $16992 \pm 59$ & $482 \pm 36$ & 66 & 0.057 & \\
\hline
\end{tabular}


Table 1 - Continued

\begin{tabular}{|c|c|c|c|c|c|c|c|c|}
\hline Abell & $\begin{array}{c}\alpha \\
(2000)\end{array}$ & $\begin{array}{c}\delta \\
(2000)\end{array}$ & $\begin{array}{c}V_{1} \\
\mathrm{~km} \mathrm{~s}^{-1}\end{array}$ & $\begin{array}{c}V_{c} \\
\mathrm{~km} \mathrm{~s}^{-1}\end{array}$ & $\begin{array}{c}\sigma_{c} \\
\mathrm{~km} \mathrm{~s}^{-1}\end{array}$ & $N_{g}$ & $A_{B}$ & Notes \\
\hline 1334 & 174.7656 & -4.3177 & $16882 \pm 49$ & $16706 \pm 102$ & $687 \pm 70$ & 45 & 0.218 & \\
\hline 1344 & 175.2210 & -10.7238 & $23213+6$ & $23300 \pm 68$ & $137+142$ & 4 & 0.125 & \\
\hline 1365 & 176.1274 & +30.8831 & $22904 \pm 41$ & $22709 \pm 78$ & $369 \pm 61$ & 22 & 0.104 & \\
\hline 1367 & 176.0090 & $\begin{array}{l}+19.9496 \\
\end{array}$ & $6276 \pm 21$ & $6562 \pm 59$ & $872 \pm 42$ & 217 & 0.100 & N3842 15K \\
\hline 1371 & 176.3290 & $\begin{array}{r}+15.4867 \\
\end{array}$ & $20348 \pm 45$ & $20475 \pm 68$ & $577 \pm 50$ & 71 & 0.188 & \\
\hline 1375 & 176.5157 & -8.2711 & $22338 \pm 50$ & $23951 \pm 285$ & $570 \pm 530$ & 4 & 0.209 & I0734 \\
\hline 1377 & 176.8394 & +55.7297 & $15457 \pm 36$ & $15465 \pm 66$ & $745 \pm 47$ & 126 & 0.047 & \\
\hline 1383 & 177.1909 & +54.5190 & $17988 \pm 51$ & $17898 \pm 85$ & $816 \pm 39$ & 92 & 0.057 & \\
\hline 1400 & 177.8067 & +55.1161 & $23684 \pm 60$ & $23819 \pm 78$ & $332 \pm 53$ & 18 & 0.058 & \\
\hline 1404 & 178.0518 & -2.8050 & $23182 \pm 46$ & $23251 \pm 74$ & $365 \pm 52$ & 24 & 0.080 & \\
\hline 1424 & 179.3710 & +5.0889 & $23150 \pm 13$ & $22708 \pm 77$ & $697 \pm 55$ & 80 & 0.089 & \\
\hline 1436 & 179.8603 & +56.4036 & $18889 \pm 36$ & $19464 \pm 71$ & $703 \pm 36$ & 97 & 0.077 & \\
\hline 1452 & 180.8684 & +51.7153 & $18229 \pm 37$ & $18608 \pm 86$ & $560 \pm 63$ & 42 & 0.098 & \\
\hline 1461 & 181.1035 & +42.5615 & $16180 \pm 27$ & $15984 \pm 66$ & $402 \pm 35$ & 37 & 0.045 & \\
\hline 1474 & 182.0659 & $\begin{array}{r}+15.0380 \\
\end{array}$ & $24327 \pm 38$ & $24135 \pm 94$ & $739 \pm 42$ & 61 & 0.138 & \\
\hline 1507 & 183.7030 & $\begin{array}{r}+59.9060 \\
\end{array}$ & $18438+30$ & $18024+50$ & $405+48$ & 65 & 0.082 & N4199 \\
\hline 1520 & 184.8960 & -13.4368 & $21260 \pm 30$ & $20853 \pm 92$ & $320 \pm 180$ & 12 & 0.238 & \\
\hline 1534 & 186.1789 & +61.4705 & $21008 \pm 7$ & $20983 \pm 64$ & $371 \pm 55$ & 33 & 0.081 & \\
\hline 1569 & 189.0342 & $\begin{array}{l}+16.6411 \\
\end{array}$ & $23597 \pm 4$ & $23814 \pm 105$ & $622 \pm 1314$ & 35 & 0.123 & I 3557 \\
\hline 1589 & 190.3230 & +18.5743 & $20955 \pm 0$ & $21643 \pm 99$ & $899 \pm 546$ & 82 & 0.090 & \\
\hline 1610 & 191.9402 & $\begin{array}{r}+30.0769 \\
\end{array}$ & $18174 \pm 0$ & $18659 \pm 62$ & $292 \pm 403$ & 22 & 0.070 & I0822 \\
\hline 1630 & 192.9729 & +4.5796 & $19355 \pm 47$ & $19529 \pm 71$ & $441 \pm 53$ & 38 & 0.159 & \\
\hline 1631 & 193.3258 & -15.5328 & $14278 \pm 3$ & $13830 \pm 43$ & $753 \pm 24$ & 305 & 0.235 & $15 \mathrm{~K}$ \\
\hline 1644 & 194.2990 & -17.4097 & $14267 \pm 49$ & $14120 \pm 61$ & $1016 \pm 49$ & 273 & 0.309 & $15 \mathrm{~K}$ \\
\hline 1648 & 194.7155 & -26.6492 & $23462 \pm 53$ & $23182 \pm 294$ & $721 \pm 382$ & 6 & 0.339 & \\
\hline 1656 & 195.0335 & +27.9767 & $6498 \pm 10$ & $6960 \pm 38$ & $1035 \pm 25$ & 713 & 0.035 & N4889 15K \\
\hline 1691 & 197.7863 & $\begin{array}{l}+39.2267 \\
\end{array}$ & $21686 \pm 16$ & $21723 \pm 79$ & $784 \pm 45$ & 97 & 0.074 & \\
\hline 1709 & 199.6266 & -21.4416 & $15429 \pm 45$ & $15700 \pm 114$ & $414 \pm 173$ & 13 & 0.585 & \\
\hline 1736 & 201.8668 & -27.3243 & $13608 \pm 65$ & $13530 \pm 95$ & $1127 \pm 54$ & 139 & 0.250 & $\mathrm{I} 425215 \mathrm{~K}$ \\
\hline 1741 & 201.1101 & +71.4055 & $22650 \pm 42$ & $22650 \pm 42$ & & 2 & 0.064 & \\
\hline 1749 & 202.3377 & $\begin{array}{l}+37.6227 \\
\end{array}$ & $16881 \pm 7$ & $16838 \pm 94$ & $707 \pm 66$ & 56 & 0.047 & I4269 \\
\hline 1767 & 204.0354 & +59.2061 & $21194 \pm 150$ & $21150 \pm 78$ & $887 \pm 31$ & 127 & 0.047 & \\
\hline 1773 & 205.5404 & $\begin{array}{r}+2.2270 \\
\end{array}$ & $23232 \pm 6$ & $23257 \pm 84$ & $839 \pm 59$ & 98 & 0.124 & \\
\hline 1775 & 205.4551 & +26.3736 & $22616 \pm 39$ & $22581 \pm 64$ & $568 \pm 60$ & 77 & 0.050 & U08669 \\
\hline 1780 & 206.1360 & +2.9538 & $23259 \pm 42$ & $23276 \pm 74$ & $590 \pm 44$ & 62 & 0.114 & \\
\hline 1795 & 207.2191 & +26.5927 & $19019 \pm 92$ & $18797 \pm 64$ & $861 \pm 56$ & 177 & 0.055 & \\
\hline 1800 & 207.3484 & $\begin{array}{l}+28.1070 \\
\end{array}$ & $22554 \pm 42$ & $22590 \pm 84$ & $767 \pm 190$ & 82 & 0.066 & U08738 \\
\hline 1809 & 208.2769 & +5.1494 & $23618 \pm 40$ & $23822 \pm 71$ & $745 \pm 30$ & 110 & 0.112 & \\
\hline 1825 & 209.5019 & +20.6318 & $18050 \pm 10$ & $18919 \pm 154$ & $1024 \pm 0$ & 44 & 0.136 & U08888 \\
\hline 1827 & 209.5256 & +21.6722 & $19917 \pm 39$ & $19743 \pm 49$ & $282 \pm 76$ & 32 & 0.136 & \\
\hline 1828 & 209.5617 & $\begin{array}{l}+18.3459 \\
\end{array}$ & $18830 \pm 20$ & $18736 \pm 94$ & $388 \pm 84$ & 17 & 0.124 & \\
\hline 1831 & 209.8131 & +27.9760 & $22777 \pm 41$ & $22644 \pm 111$ & $1176 \pm 118$ & 112 & 0.083 & \\
\hline 1836 & 210.4243 & -11.6063 & $11231 \pm 68$ & $11275 \pm 48$ & $354 \pm 37$ & 54 & 0.277 & $15 \mathrm{~K}$ \\
\hline 1873 & 212.8771 & +28.1707 & $22983 \pm 44$ & $22594 \pm 169$ & $656 \pm 0$ & 15 & 0.085 & \\
\hline 1890 & 214.4077 & $\begin{array}{r}+8.1792 \\
\end{array}$ & $17569 \pm 16$ & $17294 \pm 61$ & $550 \pm 59$ & 80 & 0.147 & N5539 \\
\hline 1898 & 215.1374 & +25.3439 & $23695 \pm 24$ & $23589 \pm 74$ & $419 \pm 111$ & 32 & 0.090 & \\
\hline 1899 & 215.4241 & +17.7520 & $16445 \pm 39$ & $15796 \pm 89$ & $646 \pm 0$ & 52 & 0.112 & \\
\hline 1904 & 215.5429 & +48.5701 & $21555 \pm 0$ & $21533 \pm 66$ & $772 \pm 31$ & 134 & 0.090 & \\
\hline 1913 & 216.8215 & $\begin{array}{l}+16.8307 \\
\end{array}$ & $15452 \pm 39$ & $15944 \pm 60$ & $636 \pm 130$ & 111 & 0.106 & I 4426 \\
\hline 1964 & 221.6482 & -8.7685 & $21313 \pm 44$ & $21313 \pm 44$ & & 2 & 0.364 & \\
\hline 1982 & 222.8104 & +30.6919 & $16494 \pm 62$ & $16775 \pm 241$ & $1325 \pm 0$ & 30 & 0.088 & \\
\hline 1983 & 223.1802 & $\begin{array}{l}+16.9036 \\
\end{array}$ & $13829 \pm 39$ & $13499 \pm 48$ & $541 \pm 27$ & 122 & 0.114 & $15 \mathrm{~K}$ \\
\hline 1991 & 223.6314 & $\begin{array}{r}+18.6420 \\
\end{array}$ & $17752 \pm 14$ & $17538 \pm 69$ & $604 \pm 57$ & 76 & 0.144 & N5778 \\
\hline 2022 & 226.0664 & +28.4963 & $16938 \pm 0$ & $17354 \pm 68$ & $607 \pm 74$ & 79 & 0.120 & \\
\hline 2028 & 227.3677 & $\begin{array}{r}+7.5563 \\
\end{array}$ & $23148 \pm 35$ & $23213 \pm 89$ & $658 \pm 57$ & 54 & 0.149 & \\
\hline 2029 & 227.7337 & +5.7444 & $23401 \pm 9$ & $23186 \pm 124$ & $1222 \pm 75$ & 97 & 0.170 & I1101 \\
\hline 2040 & 228.1989 & +7.4339 & $13734 \pm 47$ & $13530 \pm 52$ & $567 \pm 39$ & 119 & 0.186 & U09767 15K \\
\hline 2052 & 229.1856 & +7.0213 & $10314 \pm 14$ & $10406 \pm 65$ & $681 \pm 41$ & 109 & 0.160 & U09799 15K \\
\hline 2061 & 230.3360 & $\begin{array}{r}T .0606 \\
+30.6706\end{array}$ & $23706 \pm 34$ & $23436 \pm 66$ & $851 \pm 28$ & 166 & 0.091 & \\
\hline 2063 & 230.7728 & +8.6092 & $10269 \pm 36$ & $10474 \pm 78$ & $930 \pm 57$ & 141 & 0.147 & $15 \mathrm{~K}$ \\
\hline 2065 & 230.6005 & +27.7141 & $20740 \pm 15$ & $21667 \pm 109$ & $1286 \pm 140$ & 137 & 0.174 & \\
\hline 2067 & 230.7852 & +30.8771 & $22061 \pm 54$ & $22346 \pm 69$ & $621 \pm 31$ & 80 & 0.093 & \\
\hline 2079 & 231.9383 & +28.9286 & $19616 \pm 33$ & $19828 \pm 68$ & $676 \pm 41$ & 97 & 0.096 & U09861 \\
\hline 2089 & 233.2082 & $\begin{array}{r}+28.0392 \\
\end{array}$ & $22073 \pm 21$ & $22072 \pm 80$ & $593 \pm 57$ & 55 & 0.123 & \\
\hline 2092 & 233.3144 & +31.1446 & $20410 \pm 45$ & $19979 \pm 53$ & $470 \pm 49$ & 77 & 0.110 & \\
\hline 2107 & 234.9127 & +21.7821 & $12611 \pm 60$ & $12429 \pm 66$ & $629 \pm 46$ & 90 & 0.247 & U09958 15K \\
\hline 2147 & 240.5710 & +15.9743 & $10617 \pm 42$ & $10978 \pm 54$ & $1033 \pm 33$ & 359 & 0.139 & U10143 15K \\
\hline 2151 & 241.1490 & +17.7217 & $10552 \pm 23$ & $10937 \pm 46$ & $842 \pm 30$ & 335 & 0.205 & N6041A $15 \mathrm{~K}$ \\
\hline 2152 & 241.3720 & $\begin{array}{l}+16.4364 \\
\end{array}$ & $13249 \pm 59$ & $13243 \pm 50$ & $456 \pm 62$ & 81 & 0.172 & U10187 15K \\
\hline 2162 & 243.1489 & $\begin{array}{r}1 \\
+29.4843\end{array}$ & $9581 \pm 5$ & $9679 \pm 57$ & $435 \pm 37$ & 57 & 0.161 & N6086 15K \\
\hline 2184 & 245.2711 & +50.2223 & $16017 \pm 6$ & $16361 \pm 124$ & $634 \pm 357$ & 26 & 0.092 & \\
\hline 2197 & 247.4377 & $\begin{array}{r}1 \\
+40.8118\end{array}$ & $8825 \pm 8$ & $9093 \pm 41$ & $615 \pm 21$ & 216 & 0.031 & N6173 15K \\
\hline 2198 & 246.9807 & +43.9480 & $17485 \pm 32$ & $17581 \pm 69$ & $208 \pm 60$ & 9 & 0.031 & \\
\hline 2199 & 247.1602 & $\begin{array}{r}+39.5508 \\
\end{array}$ & $9317 \pm 10$ & $9088 \pm 38$ & $819 \pm 32$ & 454 & 0.050 & N6166 15K \\
\hline 2241 & 254.7856 & +32.5005 & $18467 \pm 60$ & $18573 \pm 57$ & $275 \pm 42$ & 23 & 0.130 & \\
\hline 2247 & 253.2003 & +81.6328 & $11135 \pm 18$ & $11583 \pm 75$ & $353 \pm 59$ & 22 & 0.274 & U10638 15K \\
\hline
\end{tabular}


Table 1 - Continued

\begin{tabular}{|c|c|c|c|c|c|c|c|c|}
\hline Abell & $\begin{array}{c}\alpha \\
(2000)\end{array}$ & $\begin{array}{c}\delta \\
(2000)\end{array}$ & $\begin{array}{c}V_{1} \\
\mathrm{~km} \mathrm{~s}^{-1}\end{array}$ & $\begin{array}{c}V_{c} \\
\mathrm{~km} \mathrm{~s}^{-1}\end{array}$ & $\begin{array}{c}\sigma_{c} \\
\mathrm{~km} \mathrm{~s}^{-1}\end{array}$ & $N_{g}$ & $A_{B}$ & Notes \\
\hline 2248 & 254.7646 & +77.0476 & $19481 \pm 60$ & $19128 \pm 306$ & $1224 \pm 1758$ & 16 & 0.168 & \\
\hline 2250 & 257.7555 & +39.6903 & $19721 \pm 40$ & $19416 \pm 160$ & $699 \pm 0$ & 19 & 0.186 & \\
\hline 2256 & 256.1144 & +78.6401 & $17808 \pm 53$ & $17461 \pm 75$ & $1301 \pm 42$ & 299 & 0.227 & U10726 \\
\hline 2271 & 259.5696 & $\begin{array}{r}1 \\
+78.0182\end{array}$ & $16962 \pm 45$ & $17133 \pm 162$ & $538 \pm 135$ & 11 & 0.183 & \\
\hline 2293 & 270.5712 & +57.5480 & $21151 \pm 35$ & $20782 \pm 337$ & $754 \pm 0$ & 5 & 0.225 & \\
\hline 2308 & 278.5358 & +70.9552 & $23970 \pm 39$ & $23970 \pm 39$ & $\ldots$ & 2 & 0.287 & \\
\hline 2309 & 279.7795 & +77.8395 & $15393 \pm 49$ & $15393 \pm 49$ & & 3 & 0.318 & \\
\hline 2325 & 307.5258 & -24.9837 & $24129 \pm 51$ & $24154 \pm 146$ & $293 \pm 0$ & 4 & 0.225 & \\
\hline 2331 & 314.5507 & -7.7599 & $23681 \pm 44$ & $24092 \pm 141$ & $788 \pm 101$ & 31 & 0.348 & \\
\hline 2361 & 324.7629 & -14.3646 & $17987 \pm 54$ & $18260 \pm 46$ & $313 \pm 36$ & 46 & 0.231 & \\
\hline 2362 & 325.0611 & -14.2315 & $18379 \pm 35$ & $18238 \pm 47$ & $321 \pm 39$ & 45 & 0.242 & \\
\hline 2366 & 325.7031 & -6.8687 & $16130 \pm 31$ & $15908 \pm 69$ & $540 \pm 49$ & 61 & 0.189 & \\
\hline 2370 & 326.0999 & -19.4410 & $17728 \pm 36$ & $17783 \pm 281$ & $744 \pm 260$ & 7 & 0.158 & \\
\hline 2372 & 326.3148 & -19.9947 & $17623 \pm 39$ & $17857 \pm 231$ & $612 \pm 183$ & 7 & 0.154 & \\
\hline 2382 & 327.9820 & -15.7063 & $19704 \pm 40$ & $19170 \pm 66$ & $900 \pm 81$ & 184 & 0.248 & \\
\hline 2383 & 327.8515 & -21.0779 & $17224 \pm 54$ & $17252 \pm 33$ & $75 \pm 162$ & 5 & 0.164 & \\
\hline 2388 & 328.4138 & +8.2524 & $18202 \pm 36$ & $18202 \pm 36$ & & 1 & 0.209 & \\
\hline 2399 & 329.2572 & -7.8397 & $18630 \pm 35$ & $17370 \pm 51$ & $713 \pm 27$ & 190 & 0.168 & \\
\hline 2401 & 329.5939 & -20.1046 & $17379 \pm 48$ & $17164 \pm 83$ & $470 \pm 62$ & 32 & 0.126 & \\
\hline 2405 & 329.9260 & -17.8009 & $10961 \pm 41$ & $11045 \pm 78$ & $234 \pm 47$ & 9 & 0.179 & \\
\hline 2412 & 331.0102 & -21.4482 & $21963 \pm 42$ & $21620 \pm 370$ & $1282 \pm 281$ & 12 & 0.161 & \\
\hline 2415 & 331.3591 & -5.7424 & $17007 \pm 31$ & $17291 \pm 70$ & $722 \pm 202$ & 105 & 0.286 & \\
\hline 2457 & 338.9204 & +1.4845 & $17602 \pm 43$ & $17608 \pm 67$ & $642 \pm 53$ & 91 & 0.363 & \\
\hline 2459 & 339.1904 & -15.7347 & $21822 \pm 42$ & $21898 \pm 234$ & $620 \pm 218$ & 7 & 0.225 & \\
\hline 2462 & 339.7976 & -17.3413 & $22281 \pm 53$ & $22235 \pm 96$ & $702 \pm 67$ & 53 & 0.153 & \\
\hline 2469 & 340.1448 & +12.3115 & $18918 \pm 40$ & $18918 \pm 40$ & & 2 & 0.213 & \\
\hline 2480 & 341.4961 & -17.6258 & $20450 \pm 41$ & $21640 \pm 237$ & $823 \pm 187$ & 12 & 0.123 & \\
\hline 2492 & 342.6269 & -19.2430 & $20728 \pm 39$ & $20968 \pm 233$ & $739 \pm 336$ & 10 & 0.123 & \\
\hline 2495 & 342.5824 & +10.9033 & $23895 \pm 45$ & $23744 \pm 212$ & $638 \pm 188$ & 9 & 0.329 & \\
\hline 2511 & 344.7129 & -7.5828 & $23078 \pm 51$ & $23359 \pm 238$ & $534 \pm 816$ & 5 & 0.192 & \\
\hline 2524 & 345.7331 & +17.7498 & $24304 \pm 33$ & $23902 \pm 173$ & $627 \pm 175$ & 13 & 0.400 & \\
\hline 2525 & 346.0086 & -10.7519 & $23554 \pm 47$ & $23568 \pm 81$ & $425 \pm 48$ & 27 & 0.167 & \\
\hline 2558 & 348.1816 & +10.3619 & $19450 \pm 38$ & $19450 \pm 38$ & & 2 & 0.188 & \\
\hline 2559 & 348.2615 & -13.6233 & $23770 \pm 44$ & $23749 \pm 57$ & $163 \pm 75$ & 8 & 0.140 & \\
\hline 2572 & 349.3064 & +18.7078 & $12175 \pm 61$ & $11638 \pm 62$ & $593 \pm 36$ & 90 & 0.222 & N7578B $15 \mathrm{~K}$ \\
\hline 2589 & 350.9898 & +16.7766 & $12442 \pm 57$ & $12394 \pm 84$ & $872 \pm 60$ & 107 & 0.129 & N7647 15K \\
\hline 2593 & 351.0840 & +14.6473 & $12507 \pm 28$ & $12532 \pm 43$ & $644 \pm 23$ & 217 & 0.188 & N7649 15K \\
\hline 2618 & 353.5233 & +22.9830 & $21036 \pm 36$ & $21036 \pm 36$ & & 2 & 0.343 & \\
\hline 2622 & 353.7563 & +27.3719 & $18417 \pm 38$ & $18296 \pm 114$ & $860 \pm 121$ & 56 & 0.246 & \\
\hline 2625 & 354.4560 & +20.8091 & $17200 \pm 46$ & $18063 \pm 235$ & $1506 \pm 171$ & 41 & 0.295 & \\
\hline 2626 & 354.1276 & +21.1466 & $16559 \pm 38$ & $16505 \pm 73$ & $648 \pm 53$ & 78 & 0.270 & I5338 \\
\hline 2630 & 354.5735 & +15.6689 & $19788 \pm 39$ & $20087 \pm 68$ & $420 \pm 1336$ & 38 & 0.265 & \\
\hline 2634 & 354.6227 & +27.0305 & $9105 \pm 44$ & $9343 \pm 66$ & $919 \pm 45$ & 193 & 0.303 & N7720 15K \\
\hline 2637 & 354.7225 & +21.4644 & $21283 \pm 37$ & $21104 \pm 90$ & $361 \pm 59$ & 16 & 0.187 & \\
\hline 2644 & 355.2803 & +0.0949 & $20599 \pm 33$ & $20684 \pm 205$ & $943 \pm 50$ & 21 & 0.127 & \\
\hline 2656 & 356.1805 & -4.0123 & $23034 \pm 41$ & $22926 \pm 262$ & $695 \pm 280$ & 7 & 0.165 & \\
\hline 2657 & 356.1270 & +9.2643 & $12296 \pm 59$ & $12019 \pm 105$ & $807 \pm 52$ & 59 & 0.546 & $15 \mathrm{~K}$ \\
\hline 2660 & 356.3166 & -25.8358 & $16398 \pm 59$ & $15953 \pm 107$ & $870 \pm 71$ & 65 & 0.088 & E537-G005 \\
\hline 2665 & 357.7109 & +6.1492 & $16840 \pm 57$ & $16840 \pm 57$ & & 3 & 0.345 & \\
\hline 2666 & 357.7444 & +27.1466 & $8165 \pm 43$ & $8240 \pm 66$ & $377 \pm 47$ & 32 & 0.168 & N7768 15K \\
\hline 2670 & 358.5570 & -10.4193 & $23290 \pm 51$ & $22870 \pm 66$ & $963 \pm 34$ & 208 & 0.187 & \\
\hline 2675 & 358.9278 & +11.3429 & $22168 \pm 44$ & $21559 \pm 117$ & $372 \pm 156$ & 10 & 0.362 & \\
\hline 2678 & 359.0014 & +11.7238 & $21475 \pm 41$ & $21552 \pm 108$ & $361 \pm 156$ & 11 & 0.347 & \\
\hline 2716 & 0.7546 & -27.1355 & $20134 \pm 78$ & $19905 \pm 91$ & $683 \pm 58$ & 56 & 0.072 & \\
\hline 2717 & 0.8032 & -35.9380 & $14853 \pm 34$ & $14814 \pm 60$ & $568 \pm 40$ & 89 & 0.045 & E349-G022 15K \\
\hline 2731 & 2.4960 & -57.0216 & $9434 \pm 33$ & $9236 \pm 86$ & $564 \pm 76$ & 43 & 0.064 & N0025 15K \\
\hline 2734 & 2.8406 & -28.8548 & $18456 \pm 39$ & $18461 \pm 68$ & $843 \pm 49$ & 151 & 0.072 & E409-G025 \\
\hline 2764 & 5.1489 & -49.2132 & $21424 \pm 55$ & $21370 \pm 177$ & $924 \pm 119$ & 27 & 0.073 & E194-G006 \\
\hline 2771 & 6.1340 & -40.1253 & $20839 \pm 40$ & $20984 \pm 56$ & $285 \pm 46$ & 26 & 0.039 & \\
\hline 2793 & 8.4373 & -82.6024 & $17498 \pm 23$ & $17498 \pm 23$ & & 2 & 0.691 & \\
\hline 2799 & 9.3651 & -39.1301 & $19015 \pm 23$ & $19042 \pm 58$ & $475 \pm 40$ & 67 & 0.056 & \\
\hline 2800 & 9.4902 & -25.0741 & $18999 \pm 24$ & $18948 \pm 48$ & $397 \pm 29$ & 66 & 0.066 & \\
\hline 2806 & 10.2020 & -56.2148 & $8188 \pm 37$ & $8291 \pm 67$ & $435 \pm 78$ & 42 & 0.065 & N0215 15K \\
\hline 2810 & 10.3740 & -61.0817 & $17138 \pm 26$ & $17221 \pm 113$ & $321 \pm 0$ & 8 & 0.060 & \\
\hline 2819 & 11.5212 & -63.5555 & $22369 \pm 49$ & $22396 \pm 64$ & $445 \pm 46$ & 48 & 0.100 & \\
\hline 2824 & 12.1252 & -21.3606 & $18382 \pm 39$ & $18131 \pm 94$ & $311 \pm 104$ & 11 & 0.070 & \\
\hline 2836 & 13.2174 & -47.6247 & $23684 \pm 0$ & $23684 \pm 0$ & & 2 & 0.067 & \\
\hline 2841 & 13.7691 & -48.9383 & $19342 \pm 28$ & $19360 \pm 19$ & $38 \pm 850$ & 4 & 0.045 & \\
\hline 2854 & 15.1947 & -50.5350 & $18209 \pm 27$ & $18541 \pm 63$ & $397 \pm 45$ & 39 & 0.062 & E195-G022 \\
\hline 2859 & 15.5518 & -67.5813 & $19491 \pm 43$ & $19825 \pm 9$ & $21 \pm 0$ & 5 & 0.078 & \\
\hline 2864 & 16.0347 & -66.9799 & $20948 \pm 47$ & $20948 \pm 47$ & & 3 & 0.086 & \\
\hline 2870 & 16.9243 & -46.9087 & $6728 \pm 45$ & $6744 \pm 170$ & $1165 \pm 46$ & 47 & 0.058 & $\mathrm{I} 162515 \mathrm{~K}$ \\
\hline 2877 & 17.4784 & -45.9326 & $7216 \pm 19$ & $7335 \pm 98$ & $991 \pm 63$ & 102 & 0.051 & I1633 15K \\
\hline 2881 & 17.8089 & -17.0707 & $13208 \pm 57$ & $13674 \pm 377$ & $1510 \pm 202$ & 16 & 0.086 & $15 \mathrm{~K}$ \\
\hline 2896 & 19.5775 & -37.1047 & $9473 \pm 28$ & $9923 \pm 351$ & $995 \pm 537$ & 8 & 0.077 & E352-G038 15K \\
\hline 2923 & 23.0894 & -31.0925 & $21376 \pm 37$ & $21395 \pm 70$ & $377 \pm 50$ & 29 & 0.089 & \\
\hline
\end{tabular}


Table 1 - Continued

\begin{tabular}{|c|c|c|c|c|c|c|c|c|}
\hline Abell & $\begin{array}{c}\alpha \\
(2000)\end{array}$ & $\begin{array}{c}\delta \\
(2000)\end{array}$ & $\begin{array}{c}V_{1} \\
\mathrm{~km} \mathrm{~s}^{-1}\end{array}$ & $\begin{array}{c}V_{c} \\
\mathrm{~km} \mathrm{~s}^{-1}\end{array}$ & $\begin{array}{c}\sigma_{c} \\
\mathrm{~km} \mathrm{~s}^{-1}\end{array}$ & $N_{g}$ & $A_{B}$ & Notes \\
\hline 2954 & 28.9372 & -71.4585 & $16943 \pm 87$ & $16975 \pm 29$ & $71 \pm 43$ & 6 & 0.141 & \\
\hline 2992 & 33.7125 & -26.6601 & $17389 \pm 21$ & $17259 \pm 79$ & $463 \pm 46$ & 34 & 0.060 & \\
\hline 3004 & 34.7164 & -47.9995 & $19866 \pm 20$ & $19686 \pm 188$ & $625 \pm 192$ & 11 & 0.107 & \\
\hline 3009 & 35.5305 & -48.5645 & $19754 \pm 26$ & $19731 \pm 189$ & $846 \pm 170$ & 20 & 0.071 & \\
\hline 3027 & 37.4830 & -33.1779 & $23761 \pm 38$ & $23020 \pm 91$ & $909 \pm 40$ & 99 & 0.097 & \\
\hline 3045 & 40.9220 & -51.4615 & $22623 \pm 41$ & $22569 \pm 274$ & $727 \pm 419$ & 7 & 0.116 & \\
\hline 3074 & 44.5472 & -52.7287 & $21790 \pm 44$ & $21578 \pm 113$ & $299 \pm 107$ & 7 & 0.084 & \\
\hline 3078 & 45.1065 & -51.8475 & $21771 \pm 28$ & $21836 \pm 78$ & $500 \pm 55$ & 41 & 0.085 & \\
\hline 3089 & 47.0707 & -36.7088 & $19524 \pm 33$ & $19915 \pm 122$ & $691 \pm 105$ & 32 & 0.089 & \\
\hline 3094 & 47.8544 & -26.9315 & $20577 \pm 31$ & $20269 \pm 71$ & $762 \pm 47$ & 115 & 0.071 & E481-G006 \\
\hline 3095 & 48.1109 & -27.1403 & $19468 \pm 41$ & $19803 \pm 90$ & $607 \pm 50$ & 45 & 0.093 & E481-G012 \\
\hline 3098 & 48.4314 & -38.3037 & $23895 \pm 33$ & $24482 \pm 160$ & $679 \pm 38$ & 18 & 0.078 & E300-G019 \\
\hline 3100 & 48.4702 & -47.7930 & $18642 \pm 230$ & $18910 \pm 87$ & $338 \pm 55$ & 15 & 0.076 & \\
\hline 3104 & 48.5910 & -45.4206 & $21791 \pm 35$ & $21778 \pm 84$ & $618 \pm 57$ & 53 & 0.100 & \\
\hline 3106 & 48.6246 & -58.0968 & $19523 \pm 38$ & $19461 \pm 112$ & $355 \pm 104$ & 10 & 0.116 & E116-G013 \\
\hline 3107 & 49.0260 & -42.7534 & $19833 \pm 26$ & $19534 \pm 124$ & $483 \pm 97$ & 15 & 0.070 & \\
\hline 3109 & 49.1643 & -43.8547 & $18608 \pm 32$ & $19372 \pm 261$ & $1045 \pm 195$ & 16 & 0.061 & \\
\hline 3110 & 49.0517 & -50.9534 & $22249 \pm 33$ & $22890 \pm 270$ & $975 \pm 148$ & 13 & 0.111 & \\
\hline 3111 & 49.4382 & -45.7557 & $23044 \pm 47$ & $23201 \pm 131$ & $839 \pm 93$ & 41 & 0.123 & \\
\hline 3112 & 49.4905 & -44.2382 & $22777 \pm 56$ & $22540 \pm 104$ & $919 \pm 71$ & 78 & 0.052 & E248-G006 \\
\hline 3120 & 50.4853 & -51.3268 & $21413 \pm 44$ & $20543 \pm 444$ & $1332 \pm 469$ & 9 & 0.095 & \\
\hline 3122 & 50.5760 & -41.3282 & $20130 \pm 45$ & $19137 \pm 83$ & $869 \pm 59$ & 108 & 0.075 & \\
\hline 3123 & 50.7284 & -52.0303 & $18278 \pm 48$ & $18409 \pm 110$ & $480 \pm 173$ & 19 & 0.072 & \\
\hline 3125 & 51.9783 & -53.3717 & $17444 \pm 37$ & $18189 \pm 61$ & $614 \pm 17$ & 101 & 0.068 & \\
\hline 3128 & 52.6601 & -52.6201 & $17693 \pm 39$ & $18030 \pm 48$ & $892 \pm 42$ & 341 & 0.070 & \\
\hline 3133 & 53.0252 & -45.9300 & $21377 \pm 67$ & $21014 \pm 194$ & $726 \pm 166$ & 14 & 0.042 & \\
\hline 3135 & 53.5281 & -38.9930 & $18316 \pm 47$ & $18770 \pm 96$ & $775 \pm 49$ & 65 & 0.081 & \\
\hline 3142 & 54.1561 & -39.7948 & $20068 \pm 30$ & $19986 \pm 205$ & $918 \pm 138$ & 20 & 0.066 & \\
\hline 3144 & 54.2703 & -55.0224 & $13205 \pm 28$ & $13525 \pm 109$ & $598 \pm 86$ & 30 & 0.068 & $15 \mathrm{~K}$ \\
\hline 3151 & 55.1127 & -28.6772 & $20274 \pm 52$ & $20339 \pm 119$ & $935 \pm 72$ & 61 & 0.053 & \\
\hline 3158 & 55.8747 & -53.6924 & $18766 \pm 40$ & $17771 \pm 69$ & $1095 \pm 37$ & 252 & 0.066 & \\
\hline 3164 & 56.4457 & -57.0358 & $17823 \pm 47$ & $17647 \pm 153$ & $703 \pm 106$ & 21 & 0.118 & \\
\hline 3188 & 59.3486 & -27.1213 & $19316 \pm 22$ & $19193 \pm 181$ & $993 \pm 77$ & 30 & 0.053 & \\
\hline 3193 & 59.5564 & -52.3290 & $10103 \pm 31$ & $10188 \pm 74$ & $451 \pm 51$ & 37 & 0.058 & N1500 15K \\
\hline 3195 & 59.7288 & -35.3026 & $22525 \pm 32$ & $22525 \pm 32$ & & 3 & 0.022 & \\
\hline 3202 & 59.8714 & -53.6396 & $21090 \pm 17$ & $20830 \pm 110$ & $613 \pm 55$ & 31 & 0.085 & \\
\hline 3223 & 62.1360 & -30.8223 & $17923 \pm 29$ & $18002 \pm 87$ & $790 \pm 51$ & 81 & 0.068 & \\
\hline 3225 & 62.4046 & -59.5919 & $16994 \pm 33$ & $16740 \pm 165$ & $1226 \pm 110$ & 55 & 0.083 & \\
\hline 3231 & 62.8154 & -64.5356 & $23152 \pm 39$ & $23152 \pm 39$ & & 1 & 0.198 & \\
\hline 3266 & 67.8066 & -61.4536 & $18025 \pm 181$ & $17768 \pm 63$ & $1251 \pm 41$ & 387 & 0.088 & E118-G030 \\
\hline 3301 & 75.2054 & -38.6745 & $16297 \pm 23$ & $16231 \pm 144$ & $706 \pm 77$ & 24 & 0.093 & N1759 \\
\hline 3323 & 77.8447 & -28.9924 & $19221 \pm 47$ & $19070 \pm 59$ & $145 \pm 40$ & 6 & 0.059 & E422-G043 \\
\hline 3332 & 79.1462 & -42.2029 & $23954 \pm 45$ & $23954 \pm 45$ & & 1 & 0.109 & \\
\hline 3336 & 80.3782 & -40.8173 & $23900 \pm 34$ & $23734 \pm 57$ & $114 \pm 116$ & 4 & 0.109 & \\
\hline 3341 & 81.3970 & -31.6020 & $11024 \pm 30$ & $11279 \pm 63$ & $574 \pm 50$ & 83 & 0.083 & \\
\hline 3354 & 83.6795 & -28.6906 & $17623 \pm 60$ & $17504 \pm 60$ & $435 \pm 49$ & 52 & 0.113 & \\
\hline 3367 & 87.4238 & -24.5455 & $13432 \pm 84$ & $13480 \pm 49$ & $273 \pm 121$ & 31 & 0.129 & $15 \mathrm{~K}$ \\
\hline 3374 & 89.1790 & -21.2534 & $14237 \pm 62$ & $14552 \pm 114$ & $343 \pm 0$ & 9 & 0.264 & $15 \mathrm{~K}$ \\
\hline 3376 & 90.1709 & -40.0463 & $13829 \pm 30$ & $13831 \pm 68$ & $855 \pm 54$ & 154 & 0.223 & E307-G013 15K \\
\hline 3380 & 91.7425 & -49.4934 & $15692 \pm 34$ & $15831 \pm 165$ & $573 \pm 0$ & 12 & 0.224 & \\
\hline 3381 & 92.4740 & -33.5928 & $11493 \pm 3$ & $11358 \pm 50$ & $333 \pm 34$ & 43 & 0.160 & $15 \mathrm{~K}$ \\
\hline 3389 & 95.5888 & -64.9346 & $8263 \pm 23$ & $8052 \pm 77$ & $616 \pm 64$ & 63 & 0.333 & N2235 15K \\
\hline 3390 & 96.1659 & -37.3359 & $9283 \pm 5$ & $9818 \pm 179$ & $1062 \pm 155$ & 35 & 0.358 & \\
\hline 3391 & 96.5850 & -53.6934 & $16522 \pm 43$ & $16178 \pm 110$ & $1209 \pm 55$ & 119 & 0.408 & E161-G007 \\
\hline 3392 & 96.7778 & -35.4879 & $16379 \pm 33$ & $16513 \pm 97$ & $437 \pm 71$ & 20 & 0.288 & \\
\hline 3395 & 96.9018 & -54.4505 & $14613 \pm 29$ & $15132 \pm 70$ & $950 \pm 57$ & 181 & 0.486 & E161-G008 15K \\
\hline 3407 & 106.2470 & -49.0835 & $11780 \pm 48$ & $12819 \pm 111$ & $684 \pm 125$ & 38 & 0.361 & E207-G019 \\
\hline 3408 & 107.1236 & -49.2137 & $12483 \pm 44$ & $12623 \pm 111$ & $638 \pm 87$ & 33 & 0.397 & \\
\hline 3420 & 143.0290 & -24.8179 & $18973 \pm 21$ & $18891 \pm 121$ & $438 \pm 109$ & 13 & 0.286 & \\
\hline 3429 & 149.8525 & -24.9711 & $14044 \pm 42$ & $14768 \pm 122$ & $503 \pm 78$ & 17 & 0.261 & E499-G030 \\
\hline 3432 & 150.3548 & -33.0243 & $20598 \pm 30$ & $20588 \pm 55$ & $123 \pm 90$ & 5 & 0.513 & \\
\hline 3490 & 176.3340 & -34.4338 & $20417 \pm 36$ & $20589 \pm 101$ & $996 \pm 158$ & 97 & 0.374 & \\
\hline 3492 & 179.0938 & -33.5132 & $17125 \pm 0$ & $17174 \pm 196$ & $481 \pm 212$ & 6 & 0.407 & \\
\hline 3494 & 179.2911 & -32.1520 & $20690 \pm 39$ & $20566 \pm 104$ & $208 \pm 112$ & 4 & 0.316 & \\
\hline 3497 & 180.0257 & -31.3881 & $20489 \pm 49$ & $20413 \pm 71$ & $761 \pm 81$ & 112 & 0.309 & \\
\hline 3500 & 180.7318 & -30.1275 & $13249 \pm 20$ & $13249 \pm 20$ & & 3 & 0.313 & \\
\hline 3505 & 182.1788 & -34.4436 & $17322 \pm 27$ & $17471 \pm 91$ & $389 \pm 84$ & 18 & 0.330 & \\
\hline 3526 & 192.2039 & -41.3117 & $2904 \pm 40$ & $3218 \pm 58$ & $794 \pm 43$ & 182 & 0.492 & N4696 15K \\
\hline 3528 & 193.5930 & -29.0128 & $16377 \pm 51$ & $16330 \pm 75$ & $961 \pm 35$ & 160 & 0.334 & E443-G004 15K \\
\hline 3530 & 193.9004 & -30.3470 & $16274 \pm 81$ & $16338 \pm 103$ & $716 \pm 82$ & 48 & 0.373 & E443-G011 15K \\
\hline 3531 & 194.2873 & -32.9168 & $23105 \pm 0$ & $22910 \pm 136$ & $409 \pm 120$ & 9 & 0.359 & \\
\hline 3532 & 194.3423 & -30.3632 & $16256 \pm 34$ & $16641 \pm 82$ & $734 \pm 60$ & 79 & 0.366 & 151 \\
\hline 3537 & 195.2530 & -32.4414 & $5072 \pm 6$ & $4917 \pm 54$ & $333 \pm 30$ & 38 & 0.403 & E443-G024 15K \\
\hline 3542 & 197.1730 & -34.5754 & $10372 \pm 34$ & $10372 \pm 34$ & & 2 & 0.249 & $15 \mathrm{~K}$ \\
\hline 3548 & 198.5019 & -44.1782 & $15618 \pm 42$ & $15759 \pm 151$ & $566 \pm 124$ & 14 & 0.670 & \\
\hline 3549 & 198.5258 & -29.4249 & $22408 \pm 33$ & $22586 \pm 101$ & $227 \pm 64$ & 5 & 0.244 & \\
\hline
\end{tabular}


Table 1 - Continued

\begin{tabular}{|c|c|c|c|c|c|c|c|c|}
\hline Abell & $\begin{array}{c}\alpha \\
(2000)\end{array}$ & $\begin{array}{c}\delta \\
(2000)\end{array}$ & $\begin{array}{c}V_{1} \\
\mathrm{~km} \mathrm{~s}^{-1}\end{array}$ & $\begin{array}{c}V_{c} \\
\mathrm{~km} \mathrm{~s}^{-1}\end{array}$ & $\begin{array}{c}\sigma_{c} \\
\mathrm{~km} \mathrm{~s}^{-1}\end{array}$ & $N_{g}$ & $A_{B}$ & Notes \\
\hline 3552 & 199.7300 & -31.8179 & $15573 \pm 25$ & $15354 \pm 180$ & $903 \pm 100$ & 25 & 0.251 & \\
\hline 3553 & 199.8128 & -37.1796 & $14551 \pm 29$ & $15172 \pm 89$ & $448 \pm 90$ & 25 & 0.252 & $15 \mathrm{~K}$ \\
\hline 3554 & 199.8814 & -33.4888 & $14220 \pm 67$ & $14648 \pm 96$ & $562 \pm 75$ & 34 & 0.276 & E382-G043 15K \\
\hline 3556 & 201.0282 & -31.6708 & $14424 \pm 38$ & $14339 \pm 48$ & $657 \pm 46$ & 186 & 0.259 & E444-G025 $15 \mathrm{~K}$ \\
\hline 3557 & 201.2298 & -28.8877 & $23405 \pm 61$ & $23341 \pm 78$ & $222 \pm 230$ & 8 & 0.256 & \\
\hline 3558 & 201.9855 & -31.4963 & $13986 \pm 98$ & $14294 \pm 64$ & $1002 \pm 51$ & 240 & 0.218 & E444-G046 15K \\
\hline 3559 & 202.4626 & -29.5147 & $14116 \pm 8$ & $14134 \pm 87$ & $688 \pm 49$ & 62 & 0.241 & E444-G055 15K \\
\hline 3560 & 202.9722 & -33.2346 & $3705 \pm 9$ & $3634 \pm 75$ & $261 \pm 131$ & 12 & 0.240 & N5193 15K \\
\hline 3562 & 203.3947 & -31.6723 & $14677 \pm 38$ & $14605 \pm 94$ & $966 \pm 60$ & 105 & 0.251 & E444-G072 15K \\
\hline 3563 & 203.4536 & -42.5430 & $21202 \pm 31$ & $20264 \pm 188$ & $777 \pm 144$ & 17 & 0.462 & \\
\hline 3564 & 203.7307 & -35.0994 & $14468 \pm 44$ & $14958 \pm 155$ & $490 \pm 127$ & 10 & 0.266 & $15 \mathrm{~K}$ \\
\hline 3565 & 204.1627 & -33.9658 & $3754 \pm 50$ & $3848 \pm 48$ & $414 \pm 29$ & 73 & 0.267 & I4296 $15 \mathrm{~K}$ \\
\hline 3570 & 206.6000 & -37.9710 & $11298 \pm 36$ & $11146 \pm 71$ & $530 \pm 39$ & 55 & 0.351 & E325-G016 15K \\
\hline 3571 & 206.8684 & -32.8644 & $11563 \pm 44$ & $11718 \pm 107$ & $1128 \pm 59$ & 111 & 0.233 & E383-G076 15K \\
\hline 3572 & 207.0594 & -33.3827 & $12201 \pm 28$ & $11830 \pm 132$ & $1008 \pm 111$ & 58 & 0.235 & $15 \mathrm{~K}$ \\
\hline 3574 & 207.2720 & -30.2958 & $4538 \pm 40$ & $4673 \pm 53$ & $566 \pm 29$ & 114 & 0.261 & $\mathrm{I} 432915 \mathrm{~K}$ \\
\hline 3575 & 208.1598 & -32.8881 & $11132 \pm 19$ & $11216 \pm 131$ & $571 \pm 99$ & 19 & 0.247 & $15 \mathrm{~K}$ \\
\hline 3577 & 208.5615 & -27.8481 & $14776 \pm 49$ & $14668 \pm 94$ & $561 \pm 59$ & 35 & 0.297 & \\
\hline 3581 & 211.8730 & -27.0186 & $6531 \pm 29$ & $6506 \pm 61$ & $526 \pm 47$ & 73 & 0.263 & I $437415 \mathrm{~K}$ \\
\hline 3599 & 216.9523 & -23.5421 & $7697 \pm 44$ & $6978 \pm 292$ & $773 \pm 146$ & 7 & 0.397 & \\
\hline 3603 & 218.3521 & -31.8254 & $18052 \pm 0$ & $18128 \pm 166$ & $471 \pm 171$ & 8 & 0.379 & \\
\hline 3605 & 218.7682 & -28.4255 & $20633 \pm 33$ & $19335 \pm 254$ & $623 \pm 214$ & 6 & 0.383 & \\
\hline 3615 & 225.7287 & -80.5623 & $20356 \pm 47$ & $20356 \pm 191$ & $506 \pm 0$ & 7 & 1.333 & \\
\hline 3651 & 298.0359 & -55.0628 & $17923 \pm 28$ & $17958 \pm 86$ & $777 \pm 61$ & 81 & 0.229 & \\
\hline 3656 & 300.2082 & -38.5766 & $5972 \pm 6$ & $5835 \pm 55$ & $386 \pm 48$ & 48 & 0.313 & I4931 15K \\
\hline 3667 & 303.1139 & -56.8271 & $16621 \pm 51$ & $16558 \pm 76$ & $1028 \pm 59$ & 179 & 0.210 & I 4965 \\
\hline 3676 & 306.1021 & -40.3665 & $12124 \pm 15$ & $12153 \pm 57$ & $127 \pm 90$ & 5 & 0.184 & E340-G02: \\
\hline 3677 & 306.5982 & -33.3510 & $13850 \pm 47$ & $12388 \pm 541$ & $1210 \pm 607$ & 5 & 0.303 & $15 \mathrm{~K}$ \\
\hline 3684 & 308.8428 & -78.0939 & $23036 \pm 2$ & $23036 \pm 2$ & & 3 & 0.682 & \\
\hline 3685 & 308.0675 & -56.4277 & $18632 \pm 13$ & $18632 \pm 13$ & & 2 & 0.252 & \\
\hline 3687 & 308.2502 & -63.0292 & $22898 \pm 47$ & $22980 \pm 126$ & $358 \pm 85$ & 8 & 0.306 & \\
\hline 3698 & 308.9842 & -25.2792 & $5817 \pm 20$ & $5860 \pm 59$ & $244 \pm 60$ & 17 & 0.194 & N6936 15K \\
\hline 3703 & 310.0072 & -61.3338 & $21551 \pm 44$ & $21929 \pm 100$ & $458 \pm 76$ & 21 & 0.231 & \\
\hline 3716 & 312.9867 & -52.6300 & $14046 \pm 34$ & $13895 \pm 72$ & $609 \pm 49$ & 70 & 0.158 & E187-G026 15K \\
\hline 3731 & 315.4248 & -38.4983 & $13860 \pm 45$ & $13821 \pm 78$ & $391 \pm 47$ & 25 & 0.193 & \\
\hline 3733 & 315.4961 & -28.0596 & $10990 \pm 24$ & $11586 \pm 75$ & $743 \pm 52$ & 96 & 0.495 & N6999 15K \\
\hline 3736 & 316.2687 & -43.4192 & $14577 \pm 33$ & $14340 \pm 127$ & $311 \pm 233$ & 6 & 0.127 & E286-G041 15K \\
\hline 3741 & 317.7610 & -82.1486 & $23114 \pm 6$ & $23114 \pm 6$ & & 2 & 0.774 & \\
\hline 3742 & 316.9678 & -47.1787 & $4811 \pm 38$ & $5012 \pm 38$ & $269 \pm 29$ & 48 & 0.143 & N7014 15K \\
\hline 3744 & 316.8175 & -25.4690 & $11005 \pm 16$ & $11465 \pm 68$ & $653 \pm 37$ & 90 & 0.277 & N7016 15K \\
\hline 3747 & 317.1622 & -43.4864 & $9317 \pm 2$ & $9244 \pm 83$ & $299 \pm 50$ & 13 & 0.133 & E286-G059 15K \\
\hline 3753 & 318.6332 & -26.7703 & $21840 \pm 57$ & $21840 \pm 57$ & & 2 & 0.328 & \\
\hline 3764 & 321.5746 & -34.7851 & $23965 \pm 39$ & $22696 \pm 97$ & $781 \pm 56$ & 64 & 0.344 & \\
\hline 3771 & 322.4437 & -50.7058 & $22086 \pm 39$ & $22642 \pm 178$ & $536 \pm 920$ & 9 & 0.110 & \\
\hline 3781 & 323.6044 & -66.8459 & $17091 \pm 60$ & $17050 \pm 54$ & $122 \pm 86$ & 5 & 0.137 & \\
\hline 3782 & 323.7987 & -62.0790 & $16879 \pm 36$ & $16999 \pm 144$ & $557 \pm 190$ & 15 & 0.169 & \\
\hline 3785 & 323.5741 & -53.6369 & $22876 \pm 60$ & $23492 \pm 249$ & $897 \pm 312$ & 13 & 0.077 & \\
\hline 3796 & 324.8754 & -51.3957 & $22693 \pm 58$ & $22898 \pm 98$ & $197 \pm 0$ & 4 & 0.119 & \\
\hline 3799 & 325.7601 & -72.6601 & $13714 \pm 54$ & $13591 \pm 142$ & $493 \pm 122$ & 12 & 0.209 & \\
\hline 3806 & 326.5950 & -57.2874 & $22359 \pm 3$ & $23005 \pm 121$ & $991 \pm 58$ & 67 & 0.150 & \\
\hline 3809 & 326.7465 & -43.8992 & $18642 \pm 33$ & $18719 \pm 55$ & $677 \pm 39$ & 150 & 0.078 & \\
\hline 3816 & 327.8754 & -55.3375 & $11417 \pm 46$ & $11573 \pm 61$ & $354 \pm 61$ & 33 & 0.095 & \\
\hline 3822 & 328.7510 & -57.6579 & $23315 \pm 51$ & $22720 \pm 107$ & $942 \pm 76$ & 77 & 0.128 & \\
\hline 3825 & 329.5412 & -60.2455 & $21276 \pm 54$ & $22431 \pm 114$ & $865 \pm 70$ & 57 & 0.157 & \\
\hline 3826 & 330.1012 & -56.1786 & $22654 \pm 48$ & $22654 \pm 48$ & & 1 & 0.088 & \\
\hline 3844 & 333.3238 & -34.6668 & $21897 \pm 31$ & $21903 \pm 39$ & $154 \pm 30$ & 15 & 0.067 & \\
\hline 3851 & 334.2507 & -52.5274 & $16148 \pm 21$ & $16116 \pm 81$ & $345 \pm 64$ & 18 & 0.081 & \\
\hline 3869 & 335.1294 & -55.1250 & $11869 \pm 10$ & $12294 \pm 113$ & $253 \pm 346$ & 5 & 0.091 & N7249 15K \\
\hline 3879 & 336.9555 & -69.0236 & $19860 \pm 26$ & $20060 \pm 76$ & $474 \pm 55$ & 39 & 0.138 & \\
\hline 3880 & 336.9770 & -30.5763 & $17254 \pm 29$ & $17279 \pm 77$ & $854 \pm 41$ & 122 & 0.065 & \\
\hline 3895 & 339.6907 & -36.7444 & $18018 \pm 43$ & $17672 \pm 94$ & $532 \pm 54$ & 32 & 0.069 & \\
\hline 3897 & 339.7978 & -17.3416 & $22269 \pm 53$ & $22235 \pm 96$ & $702 \pm 67$ & 53 & 0.153 & \\
\hline 3898 & 340.0435 & -62.4152 & $22061 \pm 28$ & $22061 \pm 28$ & & 1 & 0.107 & \\
\hline 3912 & 341.5412 & -36.0013 & $20168 \pm 33$ & $20664 \pm 124$ & $596 \pm 71$ & 23 & 0.055 & \\
\hline 3925 & 342.7925 & -46.6728 & $23289 \pm 86$ & $23916 \pm 189$ & $500 \pm 194$ & 7 & 0.045 & \\
\hline 4008 & 352.6255 & -39.2813 & $16415 \pm 22$ & $16410 \pm 56$ & $401 \pm 49$ & 51 & 0.077 & \\
\hline 4038 & 356.8679 & -28.1096 & $8201 \pm 25$ & $8950 \pm 68$ & $831 \pm 35$ & 146 & 0.081 & I5353 15I \\
\hline 4049 & 357.9027 & -28.3655 & $8227 \pm 34$ & $8817 \pm 97$ & $768 \pm 53$ & 62 & 0.087 & I5362 15K \\
\hline 4059 & 359.2515 & -34.7590 & $14740 \pm 39$ & $14740 \pm 67$ & $830 \pm 37$ & 151 & 0.075 & E349-G010 15K \\
\hline
\end{tabular}

Note. - Columns: (1) Abell cluster number, (2) J2000 RA of the BCG, (3) J2000 Dec of the BCG, (4) heliocentric velocity of the BCG, (5) mean heliocentric cluster velocity, (6) cluster velocity dispersion (when $N>3$ ), (7) number of cluster galaxies used to derive the mean velocity and dispersion, (8) Schlegel et al. (1998) $A_{B}$ extinction for the cluster, and (9) notes, which gives the NCG, UGC, IC, and ESO catalogue designations of the BCGs where available, and $15 \mathrm{~K}$ denotes a BCG originally selected in the LP94 and PL95 sample. 
Table 2

Abell Clusters Not Observed

\begin{tabular}{|c|c|c|c|}
\hline Abell & $\begin{array}{c}V_{c} \\
\mathrm{~km} \mathrm{~s}^{-1}\end{array}$ & $N_{g}$ & Notes \\
\hline A0087 & $16566 \pm 75$ & 166 & No obs. \\
\hline A0126 & $16142 \pm 139$ & 16 & No obs. \\
\hline A0160 & $12854 \pm 85$ & 113 & No obs. M2 misidentified in $15 \mathrm{~K}$ sample as BCG. \\
\hline A0396 & $5301 \pm 71$ & 88 & No obs. \\
\hline A0419 & $20644 \pm 127$ & 48 & No obs. Foreground group observed in $15 \mathrm{~K}$ sample. \\
\hline A0480 & $4363 \pm 200$ & 17 & No obs. \\
\hline A0484 & $20495 \pm 246$ & 20 & No obs. \\
\hline $\mathrm{A} 0524$ & $23330 \pm 156$ & 27 & No obs. \\
\hline A0833 & $20314 \pm 161$ & 17 & No obs. \\
\hline A0842 & $10056 \pm 81$ & 24 & Non-E BCG \\
\hline A0865 & $21707 \pm 61$ & 25 & Non-E BCG \\
\hline A0930 & $17250 \pm 101$ & 81 & No obs. \\
\hline A1218 & $24017 \pm 87$ & 23 & Non-E BCG \\
\hline A 1270 & $20681 \pm 67$ & 66 & Non-E BCG \\
\hline A 1275 & $18819 \pm 64$ & 32 & No obs. \\
\hline A1356 & $21735 \pm 346$ & 14 & No obs. \\
\hline A1423 & $23965 \pm 107$ & 31 & No obs. \\
\hline A1564 & $23810 \pm 81$ & 74 & No obs. \\
\hline A1638 & $18642 \pm 131$ & 4 & No obs. \\
\hline A1668 & $19192 \pm 87$ & 49 & No obs. \\
\hline A1781 & $18717 \pm 67$ & 53 & No obs. \\
\hline A1783 & $20545 \pm 54$ & 58 & No obs. \\
\hline A1837 & $20974 \pm 116$ & 36 & No obs. \\
\hline A1846 & $6329 \pm 59$ & 13 & No obs. \\
\hline A2004 & $19290 \pm 24$ & 1 & Non-E BCG \\
\hline A2020 & $14460 \pm 104$ & 16 & Non-E BCG \\
\hline A2033 & $23825 \pm 180$ & 40 & No obs. \\
\hline A2056 & $22704 \pm 147$ & 8 & Non-E BCG \\
\hline A 2122 & $19793 \pm 74$ & 111 & No obs. \\
\hline A 2124 & $19830 \pm 76$ & 110 & No obs. \\
\hline A 2168 & $18807 \pm 105$ & 12 & No obs. \\
\hline A2169 & $17352 \pm 52$ & 82 & No obs. \\
\hline A 2592 & $12082 \pm 125$ & 11 & Non-E BCG \\
\hline A2911 & $24085 \pm 84$ & 45 & No obs. Foreground group observed in $15 \mathrm{~K}$ sample. \\
\hline A2995 & $11167 \pm 51$ & 7 & Non-E BCG. Was in $15 \mathrm{~K}$ sample. \\
\hline A3108 & $18940 \pm 89$ & 17 & No obs. \\
\hline A3356 & $23035 \pm 62$ & 5 & No obs. \\
\hline A3397 & $22005 \pm 264$ & 6 & No obs. \\
\hline A3509 & $17151 \pm 146$ & 7 & No obs. \\
\hline A3524 & $22404 \pm 268$ & 22 & No obs. \\
\hline A3535 & $20203 \pm 82$ & 61 & Non-E BCG \\
\hline A3561 & $20242 \pm 168$ & 18 & No obs. \\
\hline A3566 & $15421 \pm 69$ & 48 & Non-E BCG. Was in $15 \mathrm{~K}$ sample. \\
\hline A 3578 & $11713 \pm 230$ & 40 & No obs. \\
\hline A3584 & $12362 \pm 91$ & 4 & No obs. \\
\hline A3623 & $7673 \pm 817$ & 3 & Non-E BCG \\
\hline A3626 & $20016 \pm 39$ & 1 & Non-E BCG \\
\hline A3631 & $21723 \pm 0$ & 1 & Non-E BCG \\
\hline A 3756 & $22919 \pm 152$ & 9 & No obs. \\
\hline A3831 & $20720 \pm 608$ & 4 & No obs. \\
\hline A3963 & $20980 \pm 70$ & 9 & No obs. \\
\hline A4016 & $24115 \pm 36$ & 1 & Non-E BCG \\
\hline A4053 & $21094 \pm 123$ & 70 & No obs. \\
\hline
\end{tabular}

Note. - Columns: (1) Abell cluster number, (2) mean heliocentric cluster velocity, (3) number of cluster galaxies used to derive the mean velocity, and (4) notes. 
Table 3

M2 Galaxy Sample

\begin{tabular}{|c|c|c|c|c|}
\hline Abell & $\begin{array}{c}\alpha \\
(2000)\end{array}$ & $\begin{array}{c}\delta \\
(2000)\end{array}$ & $\begin{array}{c}V_{2} \\
\mathrm{~km} \mathrm{~s}^{-1}\end{array}$ & Notes \\
\hline 0027 & 6.2335 & -20.7316 & $16241 \pm 47$ & E539-G013; BCG by total flux \\
\hline 0071 & 9.4697 & +29.6076 & $21436 \pm 31$ & \\
\hline 0074 & 9.9201 & -22.1967 & $19194 \pm 0$ & \\
\hline 0075 & 9.8897 & +21.2361 & $18494 \pm 120$ & \\
\hline 0076 & 9.8896 & +6.8146 & $11916 \pm 31$ & I1566 \\
\hline 0086 & 10.6667 & -21.8004 & $17639 \pm 49$ & \\
\hline 0102 & 12.1249 & +1.3531 & $18613 \pm 34$ & U00496 \\
\hline 0116 & 14.0482 & +0.6819 & $20086 \pm 60$ & \\
\hline 0119 & 14.1064 & -1.2625 & $11399 \pm 54$ & U00583 \\
\hline 0134 & 15.9356 & -2.4188 & $21203 \pm 32$ & \\
\hline 0147 & 17.0494 & +2.1932 & $12587 \pm 75$ & U00701 \\
\hline 0154 & 17.7623 & +17.6625 & $20107 \pm 33$ & I1634 \\
\hline 0161 & 18.8215 & +37.3651 & $21934 \pm 45$ & \\
\hline 0171 & 19.3645 & +16.3065 & $20410 \pm 41$ & \\
\hline 0174 & 20.0019 & +35.8062 & $22459 \pm 35$ & \\
\hline 0179 & 20.6370 & +19.5252 & $16374 \pm 43$ & BCG by total flux \\
\hline 0240 & 25.5183 & +7.6503 & $17557 \pm 31$ & VV $177 a$ \\
\hline 0246 & 26.1973 & +5.8316 & $21302 \pm 24$ & \\
\hline 0399 & 44.5364 & +13.0732 & $20498 \pm 32$ & \\
\hline 0400 & 44.4230 & +6.0267 & $6830 \pm 10$ & N1128 \\
\hline 0428 & 49.0694 & -19.0834 & $20420 \pm 38$ & \\
\hline 0436 & 51.7830 & +9.1889 & $19453 \pm 72$ & \\
\hline 0500 & 69.5581 & -22.2383 & $20450 \pm 0$ & \\
\hline 0533 & 75.4000 & -22.6009 & $14642 \pm 34$ & \\
\hline 0539 & 79.1556 & +6.4411 & $8318 \pm 47$ & U03274 \\
\hline 0568 & 106.9300 & +35.0603 & $22088 \pm 150$ & \\
\hline 0576 & 110.3855 & +55.7572 & $12177 \pm 100$ & BCG by total flux \\
\hline 0582 & 112.0198 & +41.9630 & $16916 \pm 36$ & \\
\hline 0592 & 115.6812 & +9.3683 & $18833 \pm 30$ & \\
\hline 0595 & 117.3640 & +52.0421 & $20514 \pm 37$ & \\
\hline 0602 & 118.3612 & +29.3592 & $18067 \pm 49$ & BCG by total flux \\
\hline 0819 & 143.0760 & +9.6983 & $22185 \pm 60$ & \\
\hline 0838 & 144.2907 & -5.0413 & $15068 \pm 42$ & \\
\hline 0841 & 144.6379 & -4.3265 & $21135 \pm 40$ & \\
\hline 0970 & 154.3435 & -10.6634 & $19483 \pm 47$ & \\
\hline 0978 & 155.1824 & -6.5330 & $16400 \pm 60$ & \\
\hline 0979 & 155.0460 & -7.8484 & $16158 \pm 17$ & \\
\hline 1032 & 157.6027 & +3.9917 & $20354 \pm 56$ & \\
\hline 1066 & 159.7778 & +5.2094 & $20310 \pm 63$ & \\
\hline 1069 & 159.9942 & -8.7903 & $19801 \pm 66$ & \\
\hline 1142 & 165.2394 & +10.5058 & $11150 \pm 43$ & N3492 \\
\hline 1145 & 165.4456 & +16.7588 & $20664 \pm 38$ & \\
\hline 1149 & 165.8353 & +7.5123 & $21410 \pm 57$ & \\
\hline 1169 & 166.9835 & +43.9187 & $17378 \pm 22$ & \\
\hline 1171 & 166.8335 & +2.9718 & $22579 \pm 40$ & BCG by total flux \\
\hline 1187 & 167.8013 & +39.5969 & $22639 \pm 0$ & \\
\hline 1190 & 167.9160 & +40.8399 & $22100 \pm 0$ & \\
\hline 1203 & 168.4483 & +40.2979 & $22070 \pm 63$ & \\
\hline 1228 & 170.4279 & +34.3631 & $10578 \pm 39$ & I2744 \\
\hline 1238 & 170.7167 & +1.1129 & $22095 \pm 64$ & \\
\hline 1317 & 173.7233 & -13.6104 & $21871 \pm 0$ & \\
\hline 1318 & 174.0151 & +55.0751 & $17155 \pm 33$ & \\
\hline 1371 & 176.3427 & +15.4951 & $20350 \pm 100$ & \\
\hline 1375 & 176.5165 & -8.2652 & $23751 \pm 52$ & I0734; BCG by total flux \\
\hline 1377 & 177.0033 & +55.7609 & $15260 \pm 150$ & \\
\hline 1383 & 177.0247 & +54.6459 & $17834 \pm 150$ & BCG by total flux \\
\hline 1436 & 180.0593 & +56.2501 & $19474 \pm 34$ & BCG by total flux \\
\hline 1452 & 180.7798 & +51.6748 & $19007 \pm 46$ & \\
\hline 1630 & 192.7873 & +4.5807 & $19943 \pm 25$ & \\
\hline 1644 & 194.4554 & -17.5457 & $14013 \pm 50$ & \\
\hline 1648 & 194.7360 & -26.6264 & $22664 \pm 58$ & \\
\hline 1656 & 194.8985 & +27.9592 & $7176 \pm 15$ & N4874 \\
\hline 1709 & 199.7237 & -21.5127 & $16250 \pm 60$ & E576-G028 \\
\hline 1736 & 201.7029 & -27.1436 & $13719 \pm 49$ & E509-G009 \\
\hline 1767 & 203.9866 & +59.2330 & $21660 \pm 100$ & \\
\hline 1773 & 205.6229 & +2.2009 & $21480 \pm 45$ & \\
\hline 1775 & 205.4604 & +26.3703 & $20789 \pm 37$ & U08669 \\
\hline 1780 & 206.1693 & +2.8616 & $23351 \pm 51$ & \\
\hline 1809 & 208.2255 & +5.1203 & $23439 \pm 46$ & \\
\hline 1825 & 209.5139 & +20.6185 & $18677 \pm 60$ & U08888 \\
\hline 1827 & 209.5400 & +21.6976 & $19817 \pm 42$ & \\
\hline 1890 & 214.3805 & +8.2081 & $17079 \pm 37$ & N5535 \\
\hline 1898 & 215.1811 & +25.1441 & $24227 \pm 31$ & \\
\hline 1982 & 222.8340 & +30.6636 & $14986 \pm 62$ & \\
\hline
\end{tabular}


Present Epoch Brightest Cluster Galaxies

Table 3 - Continued

\begin{tabular}{|c|c|c|c|c|}
\hline Abell & $\begin{array}{c}\alpha \\
(2000)\end{array}$ & $\begin{array}{c}\delta \\
(2000)\end{array}$ & $\begin{array}{c}V_{2} \\
\mathrm{~km} \mathrm{~s}^{-1}\end{array}$ & Notes \\
\hline 1983 & 223.2302 & +16.7025 & $13581 \pm 52$ & \\
\hline 2022 & 226.0306 & +28.5194 & $17183 \pm 31$ & \\
\hline 2028 & 227.3912 & +7.5148 & $23485 \pm 37$ & \\
\hline 2061 & 230.3936 & +30.7202 & $23484 \pm 42$ & \\
\hline 2065 & 230.6218 & +27.7072 & $22454 \pm 0$ & \\
\hline 2079 & 231.9355 & +28.9182 & $19541 \pm 35$ & U09861 \\
\hline 2147 & 240.5530 & +15.9078 & $10489 \pm 39$ & U10143 \\
\hline 2152 & 241.3607 & +16.4424 & $13713 \pm 0$ & U10187 \\
\hline 2197 & 246.9216 & +40.9269 & $9408 \pm 77$ & N6160 \\
\hline 2198 & 246.9654 & +43.9598 & $17702 \pm 18$ & \\
\hline 2241 & 254.7547 & +32.4943 & $18710 \pm 250$ & \\
\hline 2247 & 252.7461 & +81.5746 & $11409 \pm 98$ & U10638; BCG by total flux \\
\hline 2248 & 254.4118 & +77.0629 & $19034 \pm 0$ & \\
\hline 2250 & 257.7347 & +39.6919 & $18666 \pm 34$ & \\
\hline 2256 & 256.0550 & +78.6281 & $16818 \pm 39$ & \\
\hline 2293 & 270.3396 & +57.6506 & $21198 \pm 38$ & \\
\hline 2325 & 307.5208 & -24.9819 & $23947 \pm 50$ & \\
\hline 2331 & 314.4778 & -7.6871 & $25471 \pm 42$ & \\
\hline 2361 & 324.8019 & -14.3188 & $17924 \pm 40$ & \\
\hline 2370 & 326.1072 & -19.4740 & $17672 \pm 0$ & \\
\hline 2372 & 326.3107 & -20.0080 & $17359 \pm 32$ & \\
\hline 2382 & 327.9763 & -15.6246 & $18843 \pm 0$ & \\
\hline 2399 & 329.3729 & -7.7960 & $17400 \pm 30$ & \\
\hline 2412 & 331.1290 & -21.4996 & $21200 \pm 130$ & \\
\hline 2459 & 339.1995 & -15.7443 & $21376 \pm 0$ & BCG by total flux \\
\hline 2462 & 339.8252 & -17.3369 & $22184 \pm 75$ & \\
\hline 2492 & 342.6565 & -19.2266 & $21912 \pm 34$ & \\
\hline 2511 & 344.7271 & -7.6024 & $23409 \pm 45$ & BCG by total flux \\
\hline 2524 & 345.8252 & 17.6729 & $24399 \pm 40$ & \\
\hline 2525 & 346.0455 & -10.7735 & $24039 \pm 0$ & \\
\hline 2572 & 349.3000 & +18.7013 & $11974 \pm 37$ & N7578A \\
\hline 2618 & 353.3166 & +22.9409 & $20790 \pm 38$ & \\
\hline 2625 & 354.2523 & +20.6493 & $17538 \pm 12$ & \\
\hline 2637 & 354.6761 & +21.5009 & $21471 \pm 43$ & \\
\hline 2656 & 356.1832 & -4.0023 & $22935 \pm 47$ & \\
\hline 2657 & 356.2393 & +9.1919 & $12361 \pm 46$ & BCG by total flux \\
\hline 2678 & 358.9390 & +11.6533 & $22509 \pm 40$ & \\
\hline 2764 & 5.1297 & -49.2441 & $19834 \pm 57$ & \\
\hline 2771 & 6.1129 & -40.1361 & $21042 \pm 0$ & \\
\hline 2793 & 8.3710 & -82.6114 & $17976 \pm 65$ & \\
\hline 2799 & 9.3500 & -39.1387 & $19044 \pm 54$ & \\
\hline 2800 & 9.5307 & -25.0674 & $19969 \pm 54$ & \\
\hline 2806 & 10.0538 & -56.1542 & $8227 \pm 13$ & N0212 \\
\hline 2810 & 10.3557 & -61.0804 & $17756 \pm 21$ & \\
\hline 2819 & 11.5020 & -63.5646 & $22383 \pm 52$ & BCG by total flux \\
\hline 2824 & 12.1504 & -21.3654 & $18125 \pm 39$ & \\
\hline 2836 & 13.4048 & -47.6053 & $22683 \pm 51$ & \\
\hline 2854 & 15.2002 & -50.5490 & $18777 \pm 33$ & E195-G022 \\
\hline 2859 & 15.3283 & -67.5410 & $19940 \pm 45$ & \\
\hline 2864 & 16.0524 & -67.0099 & $21165 \pm 75$ & \\
\hline 2881 & 17.7269 & -17.1977 & $12665 \pm 38$ & \\
\hline 2923 & 23.1002 & -31.0835 & $20808 \pm 38$ & \\
\hline 2954 & 28.8930 & -71.4744 & $16926 \pm 37$ & \\
\hline 3004 & 34.7666 & -47.9751 & $19501 \pm 45$ & \\
\hline 3027 & 37.6363 & -33.0153 & $23391 \pm 0$ & \\
\hline 3074 & 44.5271 & -52.7475 & $21380 \pm 64$ & \\
\hline 3078 & 45.1189 & -51.8218 & $22151 \pm 27$ & \\
\hline 3089 & 47.1586 & -36.7471 & $18489 \pm 107$ & \\
\hline 3094 & 47.8942 & -26.8969 & $20534 \pm 60$ & \\
\hline 3098 & 48.4282 & -38.2978 & $25398 \pm 32$ & E300-G019 \\
\hline 3104 & 48.5439 & -45.4444 & $21412 \pm 43$ & \\
\hline 3106 & 48.3360 & -58.1268 & $18989 \pm 45$ & \\
\hline 3107 & 48.9867 & -42.6537 & $18599 \pm 34$ & \\
\hline 3109 & 49.1555 & -43.8844 & $19444 \pm 60$ & \\
\hline 3110 & 49.1302 & -50.9120 & $22050 \pm 100$ & \\
\hline 3120 & 50.4407 & -51.3547 & $22215 \pm 64$ & \\
\hline 3122 & 50.5850 & -41.3628 & $19245 \pm 36$ & \\
\hline 3123 & 50.7330 & -52.0532 & $18056 \pm 0$ & \\
\hline 3128 & 52.7127 & -52.5090 & $17184 \pm 60$ & \\
\hline 3133 & 53.2687 & -45.9172 & $20544 \pm 51$ & \\
\hline 3135 & 53.5141 & -39.0103 & $18886 \pm 0$ & \\
\hline 3151 & 55.1055 & -28.6776 & $20403 \pm 39$ & \\
\hline 3158 & 55.7212 & -53.6314 & $17284 \pm 54$ & E156-G008; BCG by total flux \\
\hline 3164 & 56.4810 & -57.1971 & $19067 \pm 150$ & \\
\hline 3223 & 62.1389 & -30.8106 & $18685 \pm 36$ & \\
\hline 3341 & 81.4110 & -31.5530 & $10346 \pm 54$ & \\
\hline
\end{tabular}


LAUER ET AL.

Table 3 - Continued

\begin{tabular}{|c|c|c|c|c|}
\hline Abell & $\begin{array}{c}\alpha \\
(2000)\end{array}$ & $\begin{array}{c}\delta \\
(2000)\end{array}$ & $\begin{array}{c}V_{2} \\
\mathrm{~km} \mathrm{~s}^{-1}\end{array}$ & Notes \\
\hline 3354 & 83.5789 & -28.6657 & $17737 \pm 60$ & \\
\hline 3374 & 89.2291 & -21.2517 & $14502 \pm 36$ & \\
\hline 3389 & 95.3600 & -64.9936 & $8023 \pm 3$ & N2230 \\
\hline 3391 & 96.5773 & -53.6927 & $16034 \pm 43$ & E161-G007 \\
\hline 3408 & 107.0475 & -49.1649 & $12610 \pm 40$ & \\
\hline 3420 & 142.9985 & -24.9731 & $19711 \pm 0$ & \\
\hline 3492 & 179.0675 & -33.4347 & $16855 \pm 0$ & \\
\hline 3497 & 180.0851 & -31.4336 & $20233 \pm 0$ & \\
\hline 3505 & 182.1915 & -34.4273 & $17546 \pm 25$ & \\
\hline 3531 & 194.2284 & -32.9491 & $23090 \pm 45$ & \\
\hline 3548 & 198.2548 & -44.0293 & $16429 \pm 0$ & \\
\hline 3570 & 206.6972 & -37.9079 & $11237 \pm 74$ & \\
\hline 3577 & 208.5743 & -27.8534 & $14082 \pm 39$ & \\
\hline 3603 & 218.3657 & -31.7045 & $18519 \pm 68$ & \\
\hline 3605 & 218.7790 & -28.3420 & $19527 \pm 15$ & \\
\hline 3651 & 297.9859 & -55.0043 & $18386 \pm 57$ & \\
\hline 3733 & 315.4066 & -28.0323 & $11793 \pm 13$ & N6998 \\
\hline 3764 & 321.4397 & -34.7316 & $22166 \pm 47$ & \\
\hline 3771 & 322.3297 & -50.8846 & $23980 \pm 61$ & \\
\hline 3785 & 323.5283 & -53.5721 & $23491 \pm 119$ & \\
\hline 3799 & 325.8549 & -72.6641 & $13368 \pm 70$ & \\
\hline 3822 & 328.5180 & -57.8678 & $22615 \pm 59$ & \\
\hline 3825 & 329.6099 & -60.4261 & $22528 \pm 46$ & \\
\hline 3844 & 333.3715 & -34.6691 & $21975 \pm 89$ & \\
\hline 3880 & 336.9610 & -30.5622 & $16793 \pm 39$ & \\
\hline 3895 & 339.6317 & -36.7873 & $17517 \pm 38$ & \\
\hline 3912 & 341.5176 & -36.0255 & $18889 \pm 0$ & \\
\hline 3925 & 342.7470 & -46.6853 & $23624 \pm 0$ & \\
\hline 4038 & 356.9373 & -28.1421 & $8605 \pm 30$ & I5358 \\
\hline
\end{tabular}

Note. - Columns: (1) Abell cluster number, (2) J2000 RA of M2, (3) J2000 Dec of M2, (4) heliocentric velocity of M2, (5) notes, which gives the NCG, UGC, IC, and ESO catalogue designations of the BCGs where available. M2 galaxies that exceed the luminosity of the nominal BCG at large radii are indicated. 
Table 4

Summary of Imaging Runs

\begin{tabular}{ccccc}
\hline \hline Run ID & Telescope & Detector & $\begin{array}{c}\text { FOV } \\
\left({ }^{\prime}\right)\end{array}$ & $\begin{array}{c}\text { Scale } \\
\left({ }^{\prime \prime} / \text { pixel }\right)\end{array}$ \\
\hline KP89F & $4 \mathrm{~m}$ & TI-2 & 3.99 & 0.299 \\
CT89F & $1.5 \mathrm{~m}$ & TI-2 & 3.64 & 0.273 \\
CT90F & $1.5 \mathrm{~m}$ & TI-2 & 3.64 & 0.273 \\
KP91S & $2.1 \mathrm{~m}$ & TEK1024 & 5.19 & 0.304 \\
CT91S & $1.5 \mathrm{~m}$ & TEK1024 & 7.41 & 0.434 \\
KP93F & $2.1 \mathrm{~m}$ & TEK1024 & 5.19 & 0.304 \\
CT93F & $1.5 \mathrm{~m}$ & TEK1024 & 7.41 & 0.434 \\
CT94S & $1.5 \mathrm{~m}$ & TEK1024 & 7.41 & 0.434 \\
KP94S & $2.1 \mathrm{~m}$ & T1KA & 5.19 & 0.304 \\
KP94F & $2.1 \mathrm{~m}$ & T1KA & 5.19 & 0.304 \\
CT94F & $1.5 \mathrm{~m}$ & TEK1024 & 7.41 & 0.434 \\
CT95S & $1.5 \mathrm{~m}$ & TEK2K\#4 & 14.80 & 0.434 \\
KP95S & $2.1 \mathrm{~m}$ & T1KA & 5.19 & 0.304 \\
& & & & \\
\hline
\end{tabular}

Note. - Run ID names encode the observatory $(\mathrm{CT}=\mathrm{CTIO}$, $\mathrm{KP}=\mathrm{KPNO}$ ), the year of the observations (e.g., $94=1994$ ), and the observing semester $(\mathrm{F}=$ Fall, $\mathrm{S}=$ Spring). Note that the first five runs provided data for the 15K sample presented in PL95.

Table 5

Summary of Spectroscopic Runs

\begin{tabular}{lcllcccc}
\hline \hline Run ID & Telescope & Detector & Disperser & $\begin{array}{c}\text { Slit } \\
\text { P.A. }\end{array}$ & $\begin{array}{c}\text { Spectral Range } \\
(\AA)\end{array}$ & $\begin{array}{c}\text { Dispersion } \\
(\AA / \text { pixel })\end{array}$ & $\begin{array}{c}\text { Spatial Scale } \\
(" / \text { pixel })\end{array}$ \\
\hline CT92F & $1.5-\mathrm{m}$ & GEC-10 & KPGL-3 & $90^{\circ}$ & $4351-5759$ & 2.449 & 0.398 \\
CT93S & $1.5-\mathrm{m}$ & GEC-10 & KPGL-3 & $90^{\circ}$ & $4351-5759$ & 2.447 & 0.398 \\
KP92S & $2.1-\mathrm{m}$ & TI-800 & G32 & $0^{\circ}$ & $4275-6247$ & 2.471 & $\ldots$ \\
KP92F & $2.1-\mathrm{m}$ & Ford 3KA & G32 & $0^{\circ}$ & $3344-7957$ & 2.442 & 0.76 \\
KP93S & $2.1-\mathrm{m}$ & Ford 3KA & G32 & $0^{\circ}$ & $3309-7949$ & 2.443 & 0.76 \\
CT94S & $4-\mathrm{m}$ & GEC-16 & KPGL-2 & $90^{\circ}$ & $4629-6280$ & 2.895 & 0.73 \\
KP94S & $2.1-\mathrm{m}$ & Ford 3KC & G32 & $90^{\circ}$ & $3706-7850$ & 2.440 & 0.76 \\
CT94F & $4-\mathrm{m}$ & GEC-16 & KPGL-2 & $90^{\circ}$ & $4723-6367$ & 2.891 & 0.73 \\
KP94F & $2.1-\mathrm{m}$ & Ford 3KC & G32 & $90^{\circ}$ & $3695-6852$ & 2.431 & 0.76 \\
CT95S & $4-\mathrm{m}$ & Loral 3K & KPGL-3 & $90^{\circ}$ & $3674-7351$ & 1.220 & 0.51 \\
CT95F & $4-\mathrm{m}$ & Loral 3K & KPGL-3 & $90^{\circ}$ & $3688-7357$ & 1.224 & 0.51 \\
KP95F & $2.1-\mathrm{m}$ & Ford 3KA & G32 & $90^{\circ}$ & $3536-7676$ & 2.439 & 0.76 \\
KP96S & $2.1-\mathrm{m}$ & Ford 3KB & G32 & $90^{\circ}$ & $3573-7712$ & 2.439 & 0.78 \\
CT96F & $4-\mathrm{m}$ & Loral 3K & KPGL-3 & $90^{\circ}$ & $3693-7369$ & 1.222 & 0.51 \\
& & & & & & & \\
\hline
\end{tabular}

Note. - Run ID names encode the observatory ( $\mathrm{CT}=\mathrm{CTIO}$, $\mathrm{KP}=\mathrm{KPNO}$ ), the year of the observations (e.g., $94=1994$ ), and the observing semester $(\mathrm{F}=$ Fall, $\mathrm{S}=$ Spring $)$. 
LAUER ET AL.

Table 6

Redshift Catalog

\begin{tabular}{|c|c|c|c|c|c|c|c|}
\hline $\begin{array}{l}\text { Galaxy } \\
\text { ID }\end{array}$ & $\begin{array}{c}\text { R.A. } \\
\text { (J2000) }\end{array}$ & $\begin{array}{c}\text { Dec. } \\
(\mathrm{J} 2000)\end{array}$ & $\begin{array}{c}\mathrm{v}_{\text {helio }} \\
\mathrm{km} \mathrm{s}^{-1}\end{array}$ & $\begin{array}{c}\mathrm{V}_{e r r} \\
\mathrm{~km} \mathrm{~s}^{-1}\end{array}$ & $\begin{array}{c}\text { Tonry } \\
\mathrm{R} \text { value }\end{array}$ & $\begin{array}{c}\text { Emission } \\
\text { Flag }\end{array}$ & $\begin{array}{l}\text { Observing } \\
\text { Run ID }\end{array}$ \\
\hline A0014-A & 001510.98 & -235256.2 & 19280 & 26 & 10.12 & & CT94F \\
\hline A0027-A & 002456.04 & -204353.8 & 16241 & 47 & 8.47 & & KP95F \\
\hline A0044-A & 002931.67 & +120259.0 & 21614 & 29 & 11.64 & & KP94F \\
\hline A0051-A & 003142.11 & -233845.9 & 33064 & 43 & 8.87 & & СТ95F \\
\hline A0051-B & 003141.52 & -233846.2 & 33250 & 53 & 8.04 & & CT95F \\
\hline A0071-A & 003758.74 & +293612.6 & 22044 & 33 & 12.46 & & KP94F \\
\hline A0071-B & 003752.72 & +293627.5 & 21436 & 31 & 12.67 & & KP94F \\
\hline A0074-A & 003821.67 & -222041.5 & 19013 & 46 & 8.72 & & CT95F \\
\hline A0075-B & 003928.60 & +211346.1 & 17610 & 42 & 10.34 & & KP95F \\
\hline A0076-1 & 003926.32 & +064402.5 & 11297 & 36 & 11.07 & & СТ94F KР92F КР94F \\
\hline A0077-A & 004028.35 & +293320.9 & 21424 & 35 & 11.69 & & KP94F \\
\hline A0077-X & & & 21577 & 69 & 3.79 & & KP94F \\
\hline A0085-A & 004150.45 & -091812.3 & 16581 & 56 & 7.90 & & KP95F \\
\hline A0086-A & 004242.30 & -214628.1 & 18481 & 41 & 9.88 & & KP95F \\
\hline A0086-B & 004240.00 & -214801.3 & 17639 & 49 & 8.16 & & KP95F \\
\hline A0087-A & 004310.10 & -095142.3 & 15118 & 41 & 9.75 & & KP95F \\
\hline A0093-A & 004400.32 & -182754.2 & 31116 & 37 & 10.92 & & KP94F \\
\hline A0102-A & 004831.23 & +012117.1 & 18946 & 37 & 11.39 & & KP94F \\
\hline A0114-A & 005407.50 & -214251.4 & 17290 & 45 & 8.77 & & KP95F \\
\hline A0116-A & 005546.44 & +003841.9 & 20100 & 42 & 10.94 & & KP95F \\
\hline A0117-A & 005551.89 & -095908.5 & 16453 & 47 & 9.75 & & KP95F \\
\hline A0119-1 & 005616.13 & $-01 \quad 1522.1$ & 13357 & 39 & 10.63 & & СТ94F KР94F KР92F \\
\hline A0126-A & 010015.94 & -141237.4 & 11456 & 33 & 9.96 & & $\mathrm{KP} 94 \mathrm{~F}$ \\
\hline A0126-B & 005953.92 & -141440.7 & 16448 & 39 & 11.00 & & KP94F \\
\hline A0133-A & 010241.73 & -215255.2 & 17041 & 50 & 8.30 & & KP95F \\
\hline A0134-A & 010239.08 & -024146.3 & 20639 & 31 & 12.96 & & KP94F \\
\hline A0147-1 & 010837.90 & +021606.1 & 13167 & 55 & 8.31 & & KP95F KP92F \\
\hline A0150-A & 010918.44 & +131007.5 & 17754 & 38 & 11.43 & & KP95F \\
\hline A0151-A & 010851.11 & -152425.8 & 15936 & 49 & 9.26 & & KP95F \\
\hline A0152-A & $01 \quad 1003.23$ & +135840.2 & 17491 & 35 & 11.03 & & KP95F \\
\hline A0154-A & 011103.54 & +173906.3 & 18236 & 39 & 10.86 & & KP94F \\
\hline A0158-A & 011145.30 & +165310.2 & 18958 & 38 & 11.47 & & KP95F \\
\hline A0160-1 & 011259.76 & +152929.3 & 13147 & 36 & 10.23 & & KP94F \\
\hline A0161-A & 011522.39 & +372022.6 & 22185 & 45 & 8.94 & & KP95F \\
\hline A0168-A & 011457.70 & +002552.1 & 13555 & 54 & 8.46 & & KP95F KP92F \\
\hline A0170-A & 011553.94 & +131112.5 & 9843 & 30 & 12.72 & & KP95F \\
\hline A0171-A & $\begin{array}{llll}01 & 17 & 17.96\end{array}$ & +161554.9 & 20718 & 45 & 10.20 & & KP95F \\
\hline A0174-A & 012016.25 & +354825.9 & 22490 & 43 & 9.26 & & KP95F \\
\hline A0179-D & 012210.86 & +1937 13.9 & 15618 & 39 & 11.30 & & KP95F \\
\hline A0189-1 & 012326.33 & +014217.8 & 10210 & 33 & 11.06 & & СТ94F КР94F СТ96F \\
\hline A0193-1 & 012507.54 & +084157.0 & 14654 & 61 & 8.06 & & КР94F СТ96F СТ94F \\
\hline A0194-1 & 012558.84 & -012021.8 & 5303 & 38 & 9.54 & & СТ94F СТ96F КР94F \\
\hline A0194-1a & $\ldots$ & $\ldots$ & 5468 & 33 & 8.74 & & CT94F \\
\hline A0195-1 & 012654.75 & +19 1250.1 & 12943 & 52 & 8.53 & & KP95F KP92F \\
\hline A0208-A & 013132.96 & +003321.0 & 23802 & 41 & 10.06 & & KP95F \\
\hline A0225-A & 013849.03 & +184930.2 & 20819 & 41 & 9.96 & & KP95F \\
\hline A0237-A & 014043.02 & +001854.0 & 25203 & 27 & $\ldots$ & $\mathrm{E}$ & KP95F \\
\hline A0237-D & $0140 \quad 49.19$ & +000525.6 & 25190 & 41 & 8.23 & & KP95F \\
\hline A0240-A & 014206.06 & $\begin{array}{r}073952.6 \\
\end{array}$ & 18253 & 36 & 11.51 & & KP95F \\
\hline A0245-B & 014408.19 & +061858.3 & 23391 & 34 & 10.08 & & KР94F \\
\hline A0246-A & 014443.48 & +054842.8 & 22691 & 51 & 8.12 & & KP95F \\
\hline A0257-A & 014908.43 & +135746.3 & 21143 & 42 & 9.84 & & KP94F \\
\hline A0260-A & 015042.96 & +330454.8 & 10647 & 40 & 9.78 & & KP92S KP95F \\
\hline A0261-1 & 015126.48 & -021511.5 & 13974 & 43 & 10.90 & & KP95F KP92F \\
\hline A0262-1 & 015246.28 & +360905.3 & 4824 & 50 & 8.00 & & KP92S KP95F \\
\hline A0267-A & 015315.22 & +010219.5 & 17963 & 34 & 10.49 & & KP94F \\
\hline A0268-A & 015249.24 & -010006.5 & 17917 & 29 & 13.09 & & KP94F \\
\hline A0271-A & 015406.73 & +013952.9 & 24814 & 31 & 12.06 & & KP94F \\
\hline A0279-A & 015616.96 & +010302.5 & 23680 & 34 & 12.05 & & KP94F \\
\hline A0292-A & 020218.94 & +190400.7 & 19423 & 48 & 8.78 & & KР95F \\
\hline A0295-1 & $02 \quad 02 \quad 17.24$ & -010740.6 & 12813 & 56 & 8.00 & & KP92F \\
\hline A0303-B & 020633.60 & -032348.8 & 17416 & 24 & $\ldots$ & $\mathrm{E}$ & KP95F \\
\hline A0311-A & 020928.48 & +194633.8 & 19580 & 43 & 9.33 & & KP94F \\
\hline A0326-A & 021339.75 & -070843.0 & 17066 & 56 & 6.55 & & KP95F \\
\hline A0347-1 & 022526.47 & +414927.1 & 5229 & 41 & 9.38 & & KP92S KP95F KP92F \\
\hline A0357-A & 022927.65 & +131557.9 & 16948 & 48 & 7.99 & & KP95F \\
\hline A0358-A & 022958.96 & -131553.6 & 16951 & 41 & 9.40 & & KP94F \\
\hline A0376-1 & 024604.04 & +365416.7 & 14562 & 41 & 9.91 & & KP95F KP92F \\
\hline A0386-A & 025002.29 & -170254.3 & 17835 & 34 & 12.71 & & KP94F \\
\hline A0386-B & 025004.25 & -171235.3 & 36376 & 41 & 9.62 & & KP94F \\
\hline A0396-B & 025733.45 & +413059.1 & 4911 & 34 & 11.43 & & KP94F \\
\hline A0396-C & 025708.91 & +413059.5 & 6343 & 36 & 10.40 & & KP94F KP95F \\
\hline A0397-1 & 025628.91 & +155458.9 & 10383 & 45 & 9.19 & & KP95F KP92F \\
\hline A0399-A & 025753.17 & $\begin{array}{r}+130149.8 \\
\end{array}$ & 21415 & 49 & 7.87 & & KP94F \\
\hline
\end{tabular}


Present Epoch Brightest Cluster Galaxies

Table 6 - Continued

\begin{tabular}{|c|c|c|c|c|c|c|c|}
\hline $\begin{array}{c}\text { Galaxy } \\
\text { ID }\end{array}$ & $\begin{array}{l}\text { R.A. } \\
\text { (J2000) }\end{array}$ & $\begin{array}{c}\text { Dec. } \\
\text { (J2000) }\end{array}$ & $\begin{array}{c}\mathrm{v}_{\text {helio }} \\
\mathrm{km} \mathrm{s}^{-1}\end{array}$ & $\begin{array}{c}\mathrm{v}_{\text {err }} \\
\mathrm{km} \mathrm{s}^{-1}\end{array}$ & $\begin{array}{c}\text { Tonry } \\
\mathrm{R} \text { value }\end{array}$ & $\begin{array}{c}\text { Emission } \\
\text { Flag }\end{array}$ & $\begin{array}{l}\text { Observing } \\
\text { Run ID }\end{array}$ \\
\hline A0401-A & 025857.84 & +133457.2 & 22215 & 58 & 7.12 & & KP95F \\
\hline A0404-A & 030134.79 & +412914.2 & 18420 & 34 & 12.12 & & KP95F \\
\hline A0404-B & $\begin{array}{lll}03 & 02 & 06.80\end{array}$ & +413536.8 & 9140 & 49 & 7.56 & & KP95F \\
\hline A0407-I & 030151.60 & +355027.4 & 14376 & 35 & 11.97 & & KP95F \\
\hline A0415-A & 030652.80 & -120622.7 & 23588 & 53 & 7.14 & & KP95F \\
\hline A0419-1 & $0308 \quad 15.84$ & -234129.0 & 20392 & 48 & 5.81 & & KP92S \\
\hline A0419-2 & 030828.73 & -233653.2 & 12246 & 43 & 7.99 & & KP92S KP95F \\
\hline A0423-A & $\begin{array}{llll}03 & 11 & 17.28\end{array}$ & -120742.1 & 23435 & 49 & 8.13 & & KP95F \\
\hline A0423-X & $\ldots$ & $\ldots$ & 24337 & 40 & 10.23 & & KP95F \\
\hline A0428-A & 031612.53 & -190455.9 & 19908 & 42 & 9.09 & & KP95F \\
\hline A0428-B & 031616.65 & -190500.1 & 20420 & 38 & 9.94 & & KP95F \\
\hline A0436-A & 032635.35 & +091102.9 & 19061 & 51 & 7.97 & & KP94F \\
\hline A0436-C & 032707.91 & +091120.0 & 19459 & 40 & 9.16 & & KP94F \\
\hline A0450-A & 034055.40 & +233531.9 & 17685 & 51 & 8.05 & & KP95F \\
\hline A0484-D & $0415 \quad 49.25$ & -074120.5 & 20670 & 59 & 6.62 & & KP95F \\
\hline A0496-1 & 043337.68 & -131539.7 & 9830 & 56 & 3.59 & & CT96F KP95F \\
\hline A0497-A & 043736.01 & +104040.7 & 28189 & 51 & 7.41 & & KP95F \\
\hline A0497-B & 043729.37 & +103937.0 & 26842 & 45 & 7.88 & & KP95F \\
\hline A0498-A & 043750.79 & +211219.0 & 17399 & 55 & 6.93 & & KP94F \\
\hline A0500-A & 043852.55 & -220639.6 & 20340 & 48 & 9.79 & & KP95F \\
\hline A0505-A & 045956.06 & +801042.3 & 16017 & 47 & 9.65 & & KP94F \\
\hline A0514-A & 044706.51 & -202852.0 & 21359 & 49 & 8.96 & & KP95F \\
\hline A0524-F & 045751.00 & -194229.1 & 22914 & 48 & 8.12 & & KP95F \\
\hline A0533-1 & $\begin{array}{lll}05 & 01 & 08.25\end{array}$ & -223458.1 & 14380 & 45 & 8.69 & & KP92S CT96F KP95F KP92F \\
\hline A0533-X & $\ldots$ & $\ldots$ & 15899 & 62 & 6.11 & & KP95F \\
\hline A0536-A & 050744.52 & -091527.0 & 44295 & 45 & 9.16 & & KP94F \\
\hline A0539-1 & 051655.12 & +063309.5 & 9707 & 43 & 10.46 & & KP92S KP95F KP92F \\
\hline A0548-B & 054921.74 & -252047.0 & 11867 & 37 & 9.40 & & KP92S KP95F \\
\hline A0550-A & 055251.12 & -210304.5 & 29829 & 54 & 7.98 & & KP95F \\
\hline A0553-A & 061241.21 & +483543.8 & 20323 & 42 & 10.71 & & KP94F \\
\hline A0555-B & 061733.37 & -171525.3 & 29742 & 62 & 3.87 & & CT94S \\
\hline A0559-A & 063933.00 & +695153.7 & 23310 & 37 & 10.22 & & KP95F \\
\hline A0564-A & 070040.43 & +695424.8 & 23188 & 34 & 10.09 & & KP94F \\
\hline A0568-A & 070741.58 & +350329.6 & 23496 & 48 & 8.01 & & KP94F \\
\hline A0569-1 & 070907.76 & +483655.3 & 5839 & 46 & 8.59 & & KP95F KP93S \\
\hline A0576-A & 072206.83 & +555229.3 & 12093 & 34 & 10.42 & & KP92S KP95F \\
\hline A0582-A & 072800.83 & +415506.5 & 17517 & 36 & 12.38 & & KP94S KP94F \\
\hline A0592-A & 074243.49 & +092205.8 & 18833 & 30 & 14.64 & & KP94F \\
\hline A0592-C & 074252.99 & +09 1651.2 & 18697 & 35 & 13.36 & & KP94F \\
\hline A0595-A & 074836.83 & +521319.1 & 19282 & 36 & 12.04 & & KP94F \\
\hline A0595-B & 074927.35 & +520231.7 & 20514 & 37 & 11.44 & & KP94F \\
\hline A0600-A & 075635.81 & +634423.0 & 23302 & 34 & 10.08 & & KP94F \\
\hline A0607-A & 075737.11 & +392017.8 & 29448 & 30 & 12.03 & & KP94F \\
\hline A0612-A & $0801 \quad 05.49$ & +344939.2 & 46215 & 70 & 6.39 & & KP94F \\
\hline A0634-A & 081544.90 & +581915.8 & 8143 & 39 & 9.67 & & KP92S KP94S KP95F \\
\hline A0644-A & 081725.65 & -073046.2 & 21176 & 43 & 6.00 & & CT94S \\
\hline A0644-B & 081754.50 & -072656.2 & 21191 & 37 & 7.26 & & CT94S \\
\hline A0695-A & $0841 \quad 13.13$ & +322457.9 & 20560 & 84 & 4.71 & & KP94S \\
\hline A0757-A & 091307.82 & +474229.5 & 15415 & 40 & 10.91 & & KP95F KP94S \\
\hline A0779-1 & 091946.80 & +334458.7 & 6897 & 44 & 10.39 & & KP93S KP94F KP94S KP95F \\
\hline A0780-A & 091805.71 & -120543.9 & 16275 & 79 & 2.07 & & KP94S CT95S KP94S \\
\hline A0834-A & 094132.94 & +664237.8 & 21307 & 50 & 7.34 & & KP95F \\
\hline A0834-C & 094019.60 & +663841.9 & 17919 & 46 & 8.83 & & KP95F \\
\hline A0838-A & 093709.76 & -050228.7 & 15068 & 42 & 8.51 & & CT95S \\
\hline A0838-B & 093708.63 & -050230.1 & 15668 & 39 & 9.90 & & CT95S \\
\hline A0841-A & 093836.01 & -042006.9 & 20838 & 34 & 8.29 & & CT94S \\
\hline A0841-B & 093833.10 & -041935.4 & 21135 & 40 & 6.45 & & CT94S \\
\hline A0842-A & 093734.04 & -205134.8 & 10426 & 22 & 11.29 & & CT94S \\
\hline A0912-1 & 100109.41 & -000446.5 & 13580 & 24 & 11.27 & & KP92S CT94F CT94S \\
\hline A0912-G1 & & & 28355 & 56 & 3.38 & & KP92S \\
\hline A0957-1 & 101338.27 & -005531.3 & 13391 & 58 & 7.83 & & CT95S KP93S \\
\hline A0970-D & $1018 \quad 17.98$ & -104646.6 & 11927 & 20 & 13.89 & & CT94S \\
\hline A0978-A & 102026.57 & -063137.1 & 16216 & 42 & 9.84 & & CT95S KP94S \\
\hline A0979-A & 102019.30 & -075337.5 & 15907 & 39 & 10.24 & & CT95S \\
\hline A0993-A & 102155.96 & -045325.8 & 16300 & 52 & 8.35 & & KP96S \\
\hline A0994-A & 102237.82 & +192347.3 & 11826 & 43 & 10.04 & & KP96S \\
\hline A0999-1 & 102323.85 & +125005.8 & 9749 & 35 & 10.67 & & KP92S CT95S KP94S \\
\hline A1003-A & 102501.67 & +475029.7 & 19153 & 40 & 11.77 & & KP96S \\
\hline A1016-1 & 102707.91 & +110036.9 & 9713 & 38 & 10.06 & & KP92S CT95S KP94S \\
\hline A1020-A & 102749.60 & +102629.7 & 19612 & 40 & 10.99 & & KP94S KP96S \\
\hline A1021-A & 102925.23 & +373747.1 & 30891 & 42 & 10.90 & & KP96S \\
\hline A1021-D & 102916.99 & +373537.9 & 15116 & 78 & 3.61 & & KP96S \\
\hline A1032-A & 103010.20 & +040024.3 & 19823 & 40 & 10.07 & & KP96S \\
\hline A1060-1 & 103642.56 & -273142.9 & 3862 & 41 & 7.82 & & CT94F CT94S KP93S \\
\hline A1066-A & 103938.86 & +051030.5 & 21216 & 42 & 9.97 & & KP96S KP94S \\
\hline A1100-1 & 104845.73 & +221303.4 & 13981 & 35 & 8.82 & & KP92S \\
\hline
\end{tabular}


LAUER ET AL.

Table 6 - Continued

\begin{tabular}{|c|c|c|c|c|c|c|c|}
\hline $\begin{array}{c}\text { Galaxy } \\
\text { ID }\end{array}$ & $\begin{array}{c}\text { R.A. } \\
\text { (J2000) }\end{array}$ & $\begin{array}{c}\text { Dec. } \\
(\mathrm{J} 2000)\end{array}$ & $\begin{array}{l}\mathrm{v}_{\text {helio }} \\
\mathrm{km} \mathrm{s}^{-1}\end{array}$ & $\begin{array}{c}\mathrm{V}_{\text {err }} \\
\mathrm{km} \mathrm{s}^{-1}\end{array}$ & $\begin{array}{c}\text { Tonry } \\
\mathrm{R} \text { value }\end{array}$ & $\begin{array}{c}\text { Emission } \\
\text { Flag }\end{array}$ & $\begin{array}{c}\text { Observing } \\
\text { Run ID }\end{array}$ \\
\hline A1139-1 & 105811.02 & +013615.4 & 11515 & 29 & 11.06 & & KP92S CT94S \\
\hline A1142-2 & 110045.39 & +103311.6 & 10138 & 39 & 10.47 & & KP93S \\
\hline A1149-A & 110257.62 & +073611.2 & 21247 & 35 & 11.83 & & KP94S \\
\hline A1169-A & $\begin{array}{lll}11 & 07 & 49.44\end{array}$ & $\begin{array}{r}+435459.6 \\
\end{array}$ & 17532 & 36 & 11.64 & & KP96S KP94S \\
\hline A1171-E & $\begin{array}{lll}11 & 07 & 29.99\end{array}$ & $\begin{array}{r}+025436.4 \\
\end{array}$ & 22666 & 38 & 9.31 & & KP96S \\
\hline A1177-1 & 110944.52 & $\begin{array}{r}+214531.5 \\
\end{array}$ & 9561 & 26 & 12.18 & & KP92S \\
\hline A1179-B & 111019.87 & +235800.1 & 31861 & 35 & 12.73 & & KP96S \\
\hline A1190-A & 111143.67 & $\begin{array}{r}+404913.9\end{array}$ & 23489 & 36 & 10.67 & & KP96S \\
\hline A1203-A & 111348.25 & +401708.0 & 22655 & 38 & 10.04 & & KP96S \\
\hline A1213-1 & 111622.87 & +291508.1 & 13535 & 35 & 8.90 & & KP92S \\
\hline A1257-2 & 112531.10 & $\begin{array}{r}+353015.3 \\
\end{array}$ & 10175 & 39 & 9.73 & & KP93S \\
\hline A1267-1 & 112836.40 & $\begin{array}{r}+265419.4 \\
\end{array}$ & 9795 & 39 & 9.55 & & KP93S \\
\hline A1270-A & 112842.09 & $\begin{array}{r}+541019.3 \\
\end{array}$ & 21151 & 37 & 10.65 & & KP96S \\
\hline A1279-A & 113139.57 & +671429.1 & 16263 & 50 & 8.45 & & KP96S KP94S \\
\hline A1308-1 & 113305.09 & -040049.8 & 15535 & 41 & 9.41 & & KP92S CT95S \\
\hline A1314-1 & 113449.42 & +490438.3 & 10008 & 42 & 10.56 & & KP92S KP94S \\
\hline A1317-A & 113513.06 & -133307.0 & 21632 & 40 & 10.60 & & KP96S \\
\hline A1318-A & 113734.35 & $\begin{array}{r}+545110.3 \\
\end{array}$ & 17335 & 37 & 11.90 & & KP96S \\
\hline A1318-B & 113603.63 & $\begin{array}{r}+550430.4 \\
\end{array}$ & 17155 & 33 & 12.58 & & KP96S \\
\hline A1334-A & 113903.75 & -041903.6 & 16882 & 49 & 8.07 & & CT95S \\
\hline A1344-A & 114053.03 & -104325.7 & 23213 & 46 & 8.78 & & KP94S KP96S \\
\hline A1347-A & 114124.31 & -252331.6 & 29585 & 42 & 8.37 & & CT95S \\
\hline A1365-D & 114430.57 & +305259.3 & 22904 & 41 & 9.41 & & KP96S \\
\hline A1367-1 & $\begin{array}{lll}11 & 44 & 02.17\end{array}$ & $\begin{array}{r}+195658.7\end{array}$ & 6279 & 46 & 9.39 & & KP94S KP93S \\
\hline A1367-X & & & 6355 & 40 & 9.47 & & KP94S \\
\hline A1371-A & 114518.97 & +152912.3 & 20348 & 45 & 8.77 & & KP96S \\
\hline A1375-A & 114603.76 & -081615.8 & 22338 & 50 & 7.50 & & CT95S \\
\hline A1375-B & 114603.96 & -081554.7 & 23751 & 52 & 7.20 & & CT95S \\
\hline A1377-B & 114721.46 & +554346.8 & 15457 & 36 & 12.78 & & KP96S \\
\hline A1404-A & 115212.44 & -024818.1 & 23182 & 46 & 7.86 & & CT95S \\
\hline A1424-A & 115729.04 & +050520.0 & 23149 & 43 & 9.35 & & KP96S KP94S \\
\hline A1424-C & 115734.38 & $\begin{array}{r}050550.9\end{array}$ & 23937 & 43 & 8.14 & & KP96S \\
\hline A1436-A & 120014.24 & +561500.5 & 19474 & 34 & 11.85 & & KP96S \\
\hline A1436-I & 115926.47 & +562413.0 & 18889 & 36 & 12.56 & & KP96S \\
\hline A1452-A & $\begin{array}{ll}120328.42\end{array}$ & +514255.0 & 18229 & 37 & 12.37 & & KP96S \\
\hline A1461-A & 120424.84 & +423341.3 & 16185 & 39 & 11.09 & & KP96S KP94S \\
\hline A1474-A & 120807.79 & $\begin{array}{r}+150321.2\end{array}$ & 25566 & 37 & 9.86 & & KP96S \\
\hline A1520-A & 121935.05 & -132612.3 & 21260 & 30 & 9.52 & & CT94S \\
\hline A1526-F & 122143.87 & +134514.5 & 24529 & 44 & 9.39 & & KP96S \\
\hline A1534-A & 122442.93 & +612813.9 & 21009 & 41 & 10.00 & & KP96S KP94S \\
\hline A1569-A & 123608.20 & +163827.8 & 23596 & 48 & 8.44 & & KP94S KP96S \\
\hline A1625-A & 125133.90 & -203923.8 & 19000 & 38 & 8.49 & & CT95S \\
\hline A1625-C & 125133.79 & -203933.9 & 18960 & 45 & 7.01 & & CT95S \\
\hline A1630-A & 125153.50 & +043446.4 & 19355 & 47 & 9.53 & & KP96S \\
\hline A1631-1 & 125252.51 & -152447.5 & 4053 & 26 & 13.40 & & KP92S \\
\hline A1631-A & 125318.20 & -153158.0 & 14278 & 30 & 10.55 & & KP92S CT94S \\
\hline A1644-1 & 125711.76 & -172435.0 & 14267 & 49 & 6.84 & & KP92S \\
\hline A1648-A & 125851.73 & -263857.1 & 23462 & 53 & 7.42 & & CT95S \\
\hline A1648-B & 125856.63 & -263735.1 & 22664 & 58 & 5.31 & & CT95S \\
\hline A1656-1 & 130008.04 & $\begin{array}{r}275836.3 \\
\end{array}$ & 6499 & 50 & 9.39 & & KP96S KP93S KP94S \\
\hline A1691-A & 131108.72 & +391336.0 & 21686 & 36 & 11.57 & & KP94S KP96S \\
\hline A1709-A & 131830.39 & -212629.9 & 15429 & 45 & 8.92 & & CT95S \\
\hline A1736-1 & 132728.04 & $-27 \quad 1927.6$ & 13608 & 65 & 7.47 & & KP93S \\
\hline A1741-A & 132426.42 & +712419.8 & 22650 & 42 & 8.56 & & KP96S \\
\hline A1749-A & 132921.05 & +373721.6 & 16881 & 42 & 11.02 & & KP94S KP96S \\
\hline A1773-A & 134209.70 & +021337.1 & 23232 & 49 & 8.81 & & KP96S KP94S \\
\hline A1775-A & 134149.22 & $\begin{array}{r}+262224.9\end{array}$ & 22616 & 39 & 10.17 & & KP96S \\
\hline A1780-A & 134432.63 & $\begin{array}{r}025713.7 \\
\end{array}$ & 23259 & 42 & 9.06 & & KP96S \\
\hline A1780-B & 134440.62 & +025141.7 & 23351 & 51 & 8.15 & & KP96S \\
\hline A1783-B & 134247.69 & +553116.0 & 10584 & 28 & 12.76 & & KP96S \\
\hline A1794-A & 135023.29 & -262224.6 & 37683 & 49 & 4.82 & & CT94S \\
\hline A1794-B & 135023.59 & -262303.9 & 38352 & 56 & 4.25 & & CT94S \\
\hline A1800-A & 134923.61 & $\begin{array}{r}280625.3 \\
\end{array}$ & 22554 & 42 & 9.80 & & KP96S \\
\hline A1802-B & 135120.21 & -264503.1 & 28562 & 47 & 5.74 & & CT94S \\
\hline A1802-D & 135122.42 & -264514.5 & 29302 & 53 & 3.26 & & CT94S \\
\hline A1827-A & 135806.14 & +214020.0 & 19917 & 39 & 11.20 & & KP96S \\
\hline A1827-B & 135809.60 & +214151.2 & 19817 & 42 & 10.87 & & KP96S \\
\hline A1831-A & $1359 \quad 15.15$ & $\begin{array}{r}+275833.6 \\
\end{array}$ & 22777 & 41 & 10.22 & & KP96S \\
\hline A1836-1 & 140141.84 & -113622.7 & 11271 & 47 & 8.00 & & CT94S KP93S \\
\hline A1837-A & 140136.43 & -110743.4 & 20713 & 42 & 9.27 & & CT94S KP94S \\
\hline A1846-D & $1403 \quad 16.72$ & -252827.8 & 6789 & 47 & 7.12 & & KP96S \\
\hline A1846-E & 140319.25 & -251740.8 & 19325 & 48 & 7.48 & & KP96S \\
\hline A1873-B & 141136.38 & +281256.7 & 21758 & 39 & 10.53 & & KP96S \\
\hline A1873-D & 141130.51 & +281014.5 & 22983 & 44 & 9.31 & & KP96S \\
\hline A1890-A & 141737.85 & $\begin{array}{r}+081045.2 \\
\end{array}$ & 17569 & 42 & 10.79 & & KP96S \\
\hline A $1890-B$ & 141731.33 & $\begin{array}{r}+081229.2\end{array}$ & 17079 & 37 & 10.76 & & KP96S \\
\hline
\end{tabular}


Present Epoch Brightest Cluster Galaxies

Table 6 - Continued

\begin{tabular}{|c|c|c|c|c|c|c|c|}
\hline $\begin{array}{c}\text { Galaxy } \\
\text { ID }\end{array}$ & $\begin{array}{c}\text { R.A. } \\
\text { (J2000) }\end{array}$ & $\begin{array}{c}\text { Dec. } \\
(\mathrm{J} 2000)\end{array}$ & $\begin{array}{l}\mathrm{v}_{\text {helio }} \\
\mathrm{km} \mathrm{s}^{-1}\end{array}$ & $\begin{array}{c}\mathrm{V}_{\text {err }} \\
\mathrm{km} \mathrm{s}^{-1}\end{array}$ & $\begin{array}{c}\text { Tonry } \\
\mathrm{R} \text { value }\end{array}$ & $\begin{array}{c}\text { Emission } \\
\text { Flag }\end{array}$ & $\begin{array}{c}\text { Observing } \\
\text { Run ID }\end{array}$ \\
\hline A1899-A & 142141.78 & +174507.3 & 16445 & 39 & 11.13 & & KP96S \\
\hline A1913-A & $1427 \quad 17.15$ & +164950.7 & 15452 & 39 & 11.47 & & KP96S \\
\hline A1964-A & 144635.58 & -084606.7 & 21313 & 44 & 6.67 & & CT94S \\
\hline A1964-Ax & & & 21169 & 37 & 6.65 & & CT94S \\
\hline A1983-1 & 145243.25 & +165412.9 & 13829 & 39 & 10.32 & & KP93S \\
\hline A1991-A & 145431.54 & $\begin{array}{r}+183831.3\end{array}$ & 17752 & 48 & 8.52 & & KP94S KP96S \\
\hline A1996-A & 145731.52 & -235529.7 & 33895 & 58 & 7.78 & & CT95S \\
\hline A1996-C & 145733.62 & -235512.2 & 34404 & 50 & 7.01 & & CT95S \\
\hline A2004-E & 145826.61 & +245814.7 & 19290 & 24 & $\ldots$ & $\mathrm{E}$ & KP96S \\
\hline A2020-F & 150425.72 & +074928.5 & 14703 & 43 & 10.41 & & KP96S \\
\hline A 2025-A & 150644.54 & +342822.8 & 33424 & 62 & 9.99 & & KP96S \\
\hline A2029-A & 151056.09 & +054440.0 & 23399 & 58 & 8.15 & & KP96S KP94S \\
\hline A2040-1 & $15 \quad 12 \quad 47.74$ & +072602.0 & 13734 & 47 & 9.30 & & KP93S \\
\hline A $2052-1$ & 151644.55 & +070116.8 & 10314 & 14 & $\ldots$ & $\mathrm{E}$ & KP93S \\
\hline A2055-B & 151913.18 & +060749.5 & 13566 & 35 & 10.76 & & KP96S \\
\hline A2063-1 & $\begin{array}{lll}15 & 23 & 05.46\end{array}$ & +083633.2 & 10269 & 36 & 10.51 & & KP93S \\
\hline A2065-A & $1522 \quad 24.12$ & +274250.8 & 20740 & 41 & 10.49 & & KP94S KP96S \\
\hline A2067-A & 152326.86 & $\begin{array}{r}+310357.3 \\
\end{array}$ & 22425 & 39 & 10.05 & & KP96S \\
\hline A2079-A & 152745.20 & $\begin{array}{r}+285542.9\end{array}$ & 19619 & 40 & 10.76 & & KP94S KP96S \\
\hline A2089-A & 153249.96 & +280221.3 & 22073 & 46 & 9.36 & & KP96S KP94S \\
\hline A2107-1 & 153939.05 & +214655.7 & 12611 & 60 & 7.39 & & KP93S \\
\hline A $2124-\mathrm{A}$ & 154459.13 & +360633.5 & 19892 & 42 & 10.34 & & KP94S KP96S \\
\hline A $2143-\mathrm{A}$ & 155734.95 & +372036.1 & 28179 & 38 & 11.02 & & KP96S \\
\hline A2147-1 & 160217.04 & +155827.6 & 10617 & 42 & 9.25 & & KP93S \\
\hline A2151-1 & 160435.76 & +174318.0 & 10551 & 41 & 9.79 & & KP94S KP93S \\
\hline A2152-1 & 160529.28 & +162611.0 & 13249 & 59 & 8.08 & & KP93S \\
\hline A $2162-1$ & 161235.73 & +292903.5 & 9581 & 44 & 9.92 & & KP96S KP93S \\
\hline A2168-F & 161251.19 & +540800.2 & 18678 & 67 & 4.72 & & KP96S \\
\hline A2169-A & 161358.20 & +491121.8 & 18012 & 30 & 14.90 & & KP96S \\
\hline A2184-A & 162105.07 & +501320.2 & 16017 & 38 & 11.54 & & KP96S \\
\hline A2197-1 & 162945.04 & +404842.5 & 8826 & 43 & 9.58 & & KP96S KP93S KP94S \\
\hline A2198-B & 162755.37 & +435652.8 & 17485 & 32 & 12.63 & & KP96S \\
\hline A2199-1 & 162838.45 & +393303.0 & 9318 & 85 & 4.44 & & KP96S KP93S KP94S \\
\hline A2199-A & $\ldots$ & & 9232 & 134 & 2.30 & & KP96S \\
\hline A2247-1 & 165248.08 & +813757.9 & 11133 & 64 & 6.11 & & KP96S KP93S \\
\hline A2250-A & 171056.33 & +394130.7 & 18666 & 34 & 12.96 & & KP96S \\
\hline A2250-B & 171101.32 & +394125.0 & 19721 & 40 & 10.90 & & KP96S \\
\hline A2256-A & 170335.66 & +783743.5 & 16699 & 55 & 8.48 & & KP96S \\
\hline A2256-C & 170413.21 & +783741.0 & 16818 & 39 & 11.37 & & KP96S \\
\hline A2271-A & $17 \quad 18 \quad 16.71$ & +780105.4 & 16962 & 45 & 9.94 & & KP96S \\
\hline A2293-A & 180121.50 & +573902.0 & 21198 & 38 & 10.60 & & KP96S \\
\hline A2293-B & $\begin{array}{lll}18 & 02 & 17.08\end{array}$ & +573252.8 & 21151 & 35 & 10.70 & & KP96S \\
\hline A2293-F & 180056.63 & $\begin{array}{l}+573809.5 \\
\end{array}$ & 19997 & 58 & 5.79 & & KP96S \\
\hline A2293-H & $1801 \quad 10.57$ & $\begin{array}{r}+573839.6\end{array}$ & 21756 & 73 & 2.76 & & KP96S \\
\hline A2295-B & 180033.64 & +691318.0 & 24912 & 40 & 9.97 & & KP96S \\
\hline A2295-F & 180036.25 & +691423.0 & 30529 & 44 & 9.52 & & KP96S \\
\hline A2296-A & 175431.97 & +774220.9 & 12050 & 47 & 7.81 & & KP96S \\
\hline A $2308-A$ & 183408.60 & +705718.8 & 23970 & 39 & 9.48 & & KP96S \\
\hline A2309-A & 183907.09 & +775022.2 & 15393 & 49 & 9.26 & & KP96S \\
\hline A2319-A & 192110.20 & $\begin{array}{r}+435642.7 \\
\end{array}$ & 16379 & 54 & 7.80 & & KP95F \\
\hline A2325-A & 203006.18 & -245901.2 & 24129 & 51 & 7.39 & & CT95F \\
\hline A2325-B & 203004.98 & -245854.8 & 23947 & 50 & 6.42 & & СТ95F \\
\hline A2331-A & $2058 \quad 12.17$ & -074535.6 & 23681 & 44 & 8.40 & & KР95F \\
\hline A2360-A & 213707.67 & -150528.5 & 63030 & 57 & 6.93 & & KP95F \\
\hline A2360-J & 213744.14 & -150601.3 & 26604 & 43 & 7.67 & & KP95F \\
\hline A2361-A & 213912.45 & -141907.5 & 17924 & 40 & 11.26 & & KP95F \\
\hline A2362-A & 214014.67 & -141353.4 & 18379 & 35 & 12.57 & & KP94F \\
\hline A2366-A & 214248.75 & -065207.3 & 16130 & 31 & 14.00 & & KP94F \\
\hline A2370-A & 214423.98 & -192627.5 & 17728 & 36 & 10.83 & & KP94F \\
\hline A $2372-\mathrm{A}$ & $21 \quad 45 \quad 15.54$ & -195940.8 & 17623 & 39 & 10.82 & & KP95F \\
\hline A2381-A & 215126.16 & +022650.2 & 8034 & 31 & 11.75 & & KP94F \\
\hline A2382-A & 215155.67 & -154222.7 & 19704 & 40 & 9.73 & & KP94F \\
\hline A2388-A & 215339.32 & +081508.8 & 18202 & 36 & 10.47 & & KP94F \\
\hline A2399-C & 215701.74 & -075023.0 & 18630 & 35 & 12.31 & & KP94F \\
\hline A2401-A & 215822.53 & -200616.4 & 17379 & 48 & 8.90 & & KP95F \\
\hline A $2405-\mathrm{A}$ & 215942.25 & -174803.3 & 10961 & 41 & 8.76 & & KP95F \\
\hline A2405-D & 215859.77 & -175246.5 & 28081 & 42 & $\ldots$ & $\mathrm{E}$ & KP95F \\
\hline A2405-I & 215945.93 & -175342.2 & 11499 & 48 & $\ldots$ & $\mathrm{E}$ & KP95F \\
\hline A2412-A & 220402.45 & -212653.7 & 21963 & 42 & 9.18 & & KP95F \\
\hline A2415-A & $\begin{array}{lll}22 & 05 & 26.19\end{array}$ & -054432.6 & 17007 & 31 & 14.48 & & KP94F \\
\hline A2457-A & 223540.89 & $\begin{array}{r}012904.2 \\
\end{array}$ & 17602 & 43 & 10.46 & & KP95F \\
\hline A2459-D & 223645.69 & -154405.0 & 21822 & 42 & 9.51 & & KP95F \\
\hline A2462-A & 223911.42 & -172028.8 & 22281 & 53 & 7.96 & & KP95F \\
\hline A 2469-A & 224034.75 & +121841.3 & 18918 & 40 & 9.67 & & KP95F \\
\hline A2480-A & 224559.07 & -173732.8 & 20450 & 41 & 11.12 & & KP94F \\
\hline A2492-A & 225030.45 & -19 1434.7 & 20728 & 39 & 10.47 & & KP95F \\
\hline
\end{tabular}


LAUER ET AL.

Table 6 - Continued

\begin{tabular}{|c|c|c|c|c|c|c|c|}
\hline $\begin{array}{l}\text { Galaxy } \\
\text { ID }\end{array}$ & $\begin{array}{l}\text { R.A. } \\
\text { (J2000) }\end{array}$ & $\begin{array}{c}\text { Dec. } \\
\text { (J2000) }\end{array}$ & $\begin{array}{c}\mathrm{v}_{\text {helio }} \\
\mathrm{km} \mathrm{s}^{-1}\end{array}$ & $\begin{array}{c}\mathrm{v}_{\text {err }} \\
\mathrm{km} \mathrm{s}^{-1}\end{array}$ & $\begin{array}{c}\text { Tonry } \\
\mathrm{R} \text { value }\end{array}$ & $\begin{array}{c}\text { Emission } \\
\text { Flag }\end{array}$ & $\begin{array}{c}\text { Observing } \\
\text { Run ID }\end{array}$ \\
\hline A2495-A & 225019.77 & +105411.9 & 23895 & 45 & 8.03 & & KP95F \\
\hline A2506-1 & 225637.54 & +131846.7 & 38521 & 44 & 8.55 & & KP94F \\
\hline A2511-B & 225851.10 & -073458.1 & 23078 & 51 & 6.91 & & KP95F \\
\hline A2525-A & 230402.07 & -104506.9 & 23554 & 47 & 8.71 & & KP95F \\
\hline A2525-E & 230353.28 & -104039.2 & 23501 & 44 & 8.22 & & KP95F \\
\hline A2558-A & 231243.59 & +102142.7 & 19450 & 38 & 10.83 & & KP95F \\
\hline A2559-A & $\begin{array}{lll}23 & 13 & 02.77\end{array}$ & -133724.0 & 23770 & 44 & 8.95 & & KP95F \\
\hline A 2572-A & $\begin{array}{llll}23 & 17 & 13.54\end{array}$ & +184228.2 & 12175 & 61 & 7.43 & & KP92F \\
\hline A2589-1 & 232357.55 & +164635.8 & 12442 & 57 & 7.45 & & KP92F \\
\hline A2593-1 & 232420.15 & +143850.4 & 12525 & 46 & 9.51 & & KP92F \\
\hline A2596-A & 232508.22 & -232405.0 & 26829 & 38 & 10.12 & & KP95F \\
\hline A2596-B & $23 \quad 2503.27$ & -232335.4 & 26191 & 48 & 8.68 & & KP95F \\
\hline A2618-A & 233405.60 & +225858.9 & 21036 & 36 & 12.66 & & KP94F \\
\hline A2618-B & 233315.99 & +225627.3 & 20790 & 38 & 11.33 & & KP94F \\
\hline A2622-A & 233501.51 & $\begin{array}{r}+272219.0 \\
\end{array}$ & 18417 & 38 & 11.70 & & KP94F \\
\hline A2625-I & 233749.43 & +204832.7 & 17200 & 46 & 8.66 & & KP95F \\
\hline A2626-A & 233630.63 & +210847.7 & 16559 & 38 & 10.51 & & KP94F \\
\hline A2630-F & 233817.65 & +154008.0 & 19788 & 39 & 10.05 & & KР95F \\
\hline A2634-1 & 233829.44 & +270149.7 & 9105 & 44 & 10.36 & & KP94F \\
\hline A2637-A & 233853.40 & +212751.8 & 21283 & 37 & 11.57 & & KP95F \\
\hline A2637-B & 233842.26 & +213003.1 & 21471 & 43 & 9.69 & & KP95F \\
\hline A2644-A & $23 \quad 4107.27$ & +000541.5 & 20599 & 33 & 13.22 & & KP94F \\
\hline A2656-A & 234443.33 & -040044.2 & 23034 & 41 & 9.51 & & KP95F \\
\hline A2657-2 & 234430.49 & +091551.6 & 12296 & 59 & 6.41 & & KP92F \\
\hline A2660-A & $2345 \quad 15.98$ & -255008.9 & 16398 & 59 & 7.09 & & KP95F \\
\hline A2661-C & $23 \quad 4702.88$ & -102505.9 & 57163 & 98 & 4.12 & & KP95F \\
\hline A2665-A & 235050.61 & +060857.2 & 16840 & 57 & 7.14 & & KP95F \\
\hline A2666-1 & 235058.66 & +270847.6 & 8165 & 43 & 9.21 & & KP95F \\
\hline A2670-A & 235413.68 & -102509.4 & 23290 & 51 & 7.56 & & CT95F \\
\hline A2670-X1 & $\ldots$ & $\ldots$ & 21969 & 52 & 6.54 & & CT95F \\
\hline A $2670-\mathrm{X} 2$ & $\cdots$ & $\ldots$ & 23115 & 70 & 4.21 & & CT95F \\
\hline A2675-A & 235542.66 & +112034.5 & 22168 & 44 & 9.35 & & KP95F \\
\hline A2676-C & 235550.62 & +060513.8 & 17061 & 51 & 6.93 & & KP95F \\
\hline A2676-D & 235533.14 & +060726.8 & 12834 & 55 & & $\mathrm{E}$ & KP95F \\
\hline A2678-A & 235600.34 & +114325.8 & 21475 & 41 & 9.23 & & KP95F \\
\hline A2690-A & $00 \quad 0014.01$ & $-2511 \quad 12.2$ & 25403 & 32 & 7.71 & & CT94F \\
\hline A2690-B & $00 \quad 0013.40$ & -251108.7 & 25318 & 31 & 7.53 & & СТ94F \\
\hline A2691-C & 000151.72 & -030508.2 & 24212 & 54 & 7.12 & & KP95F \\
\hline A2716-A & 000301.10 & -270807.9 & 20134 & 78 & 6.50 & & KP95F \\
\hline A2716-E & $\begin{array}{lll}0 & 02 & 30.27\end{array}$ & -271747.2 & 8532 & 53 & 5.88 & & KP95F \\
\hline A $2717-1$ & $\begin{array}{llll}0 & 03 & 12.77\end{array}$ & -355616.7 & 14853 & 34 & 7.80 & & CT92F \\
\hline A2731-1 & 000959.04 & $-5701 \quad 17.7$ & 9434 & 33 & 8.48 & & CT92F \\
\hline A2734-A & 001121.74 & $-2851 \quad 17.2$ & 18456 & 39 & 7.34 & & CT94F \\
\hline A2759-A & 001838.90 & -303334.1 & 31962 & 39 & 6.21 & & СТ94F \\
\hline A 2759-B & 001839.05 & -303327.4 & 31496 & 40 & 6.63 & & СТ94F \\
\hline A2764-D & $00 \quad 2035.74$ & -491247.7 & 21424 & 55 & 7.45 & & СТ95F \\
\hline A2765-A & $00 \quad 22 \quad 07.23$ & -205359.3 & 23078 & 38 & 6.91 & & CT94F \\
\hline A $2771-A$ & 002432.16 & -400731.2 & 20839 & 40 & 10.04 & & CT96F \\
\hline A2782-A & 003016.33 & -532450.9 & 25850 & 40 & 5.34 & & CT94F \\
\hline A2793-A & 003344.94 & -823608.6 & 17498 & 23 & 13.17 & & СТ94F \\
\hline A2793-B & 003329.03 & -823641.2 & 17976 & 65 & 3.01 & & СТ94F \\
\hline A2793-H & 003403.97 & -823538.5 & 17345 & 25 & 8.95 & & СТ94F \\
\hline A 2799-A & 003727.62 & -390748.4 & 19015 & 23 & 12.85 & & СТ94F \\
\hline A2800-A & 003757.66 & -250426.8 & 18999 & 24 & 12.77 & & СТ94F \\
\hline A2806-1 & 004012.92 & -560915.0 & 8218 & 34 & 7.80 & & CT92F \\
\hline A2806-2 & 004048.47 & -561253.4 & 8188 & 37 & 7.45 & & CT92F \\
\hline A2810-A & 004129.77 & -610454.3 & 17138 & 26 & 11.13 & & СТ94F \\
\hline A2810-B & $00 \quad 4125.37$ & -610449.5 & 17756 & 21 & 12.61 & & СТ94F \\
\hline A2814-A & 004253.75 & -282811.7 & 24468 & 36 & 6.97 & & СТ94F \\
\hline A2816-A & 004544.91 & -181457.4 & 17787 & 38 & 10.26 & & KP95F \\
\hline A2819-B & 004605.08 & -633319.9 & 22369 & 49 & 7.79 & & CT95F \\
\hline A2819-D & 004600.48 & -633352.4 & 22383 & 52 & 7.08 & & CT95F \\
\hline A2824-A & 004830.05 & -212138.1 & 18382 & 39 & 10.34 & & KP95F \\
\hline A2824-B & 004836.10 & -212155.3 & 18125 & 39 & 9.83 & & KP95F \\
\hline A2836-B & 005341.81 & -473533.3 & 17312 & 57 & 2.96 & & CT95F \\
\hline A2836-C & 005337.15 & -473619.0 & 22683 & 51 & 6.61 & & СТ95F \\
\hline A2841-A & 005504.58 & -485618.0 & 19342 & 28 & 10.29 & & CT94F \\
\hline A2854-A & 010046.72 & -503206.0 & 18209 & 27 & 10.86 & & CT94F \\
\hline A2854-B & 010048.05 & -503256.4 & 18777 & 33 & 7.47 & & CT94F \\
\hline A2859-A & $\begin{array}{lll}01 & 02 & 12.42\end{array}$ & -673452.8 & 19491 & 43 & 9.44 & & СТ96F \\
\hline A2864-A & $\begin{array}{lll}01 & 04 & 08.32\end{array}$ & -665847.7 & 20948 & 47 & 7.93 & & СТ96F \\
\hline A2870-1 & $0107 \quad 41.84$ & -465431.2 & 6728 & 45 & 5.58 & & CT92F \\
\hline A2877-1 & 010954.82 & -455557.3 & 7226 & 57 & 6.46 & & СТ92F СТ95F \\
\hline A2881-3 & 011114.14 & -170414.5 & 13253 & 43 & 9.97 & & СТ94F KР94F КР92F \\
\hline A2889-A & 011429.96 & -482827.0 & 6978 & 34 & 6.30 & & CT94F \\
\hline A2896-1 & $01 \quad 1818.60$ & -370617.0 & 9473 & 28 & 9.20 & & CT92F \\
\hline
\end{tabular}


Table 6 - Continued

\begin{tabular}{|c|c|c|c|c|c|c|c|}
\hline $\begin{array}{c}\text { Galaxy } \\
\text { ID }\end{array}$ & $\begin{array}{l}\text { R.A. } \\
\text { (J2000) }\end{array}$ & $\begin{array}{c}\text { Dec. } \\
\text { (J2000) }\end{array}$ & $\begin{array}{c}\mathrm{v}_{\text {helio }} \\
\mathrm{km} \mathrm{s}^{-1}\end{array}$ & $\begin{array}{c}\mathrm{v}_{\text {err }} \\
\mathrm{km} \mathrm{s}^{-1}\end{array}$ & $\begin{array}{c}\text { Tonry } \\
\mathrm{R} \text { value }\end{array}$ & $\begin{array}{c}\text { Emission } \\
\text { Flag }\end{array}$ & $\begin{array}{l}\text { Observing } \\
\text { Run ID }\end{array}$ \\
\hline A2911-A & 012444.69 & -380742.3 & 5791 & 29 & 8.25 & & CT92F \\
\hline A2911-B & $\begin{array}{lll}01 & 25 & 12.19\end{array}$ & -380538.0 & 5931 & 28 & 8.44 & & CT92F \\
\hline A2913-A & 012803.88 & -340658.2 & 40278 & 40 & 6.71 & & СТ94F \\
\hline A2923-A & 013221.45 & -310533.0 & 21376 & 37 & 10.45 & & СТ96F \\
\hline A2923-X & & & 21188 & 50 & 6.85 & & СТ96F \\
\hline A2955-A & 015701.88 & -170123.8 & 28311 & 38 & 10.46 & & KP94F \\
\hline A2992-A & 021451.01 & -263936.4 & 17389 & 21 & 13.50 & & CT94F \\
\hline A3004-A & 021851.93 & -475958.1 & 19866 & 20 & 13.22 & & СТ94F \\
\hline A3009-A & $\begin{array}{lll}02 & 22 & 07.32\end{array}$ & -483352.2 & 19754 & 26 & 11.50 & & СТ94F \\
\hline A3027-A & 022955.93 & -331040.4 & 23761 & 38 & 9.51 & & СТ96F \\
\hline A3045-A & 024341.29 & -512741.3 & 22623 & 41 & 6.85 & & СТ94F \\
\hline A3047-A & $02 \quad 45 \quad 13.41$ & -462720.1 & 28321 & 54 & 4.86 & & CT94F \\
\hline A3052-A & 024700.16 & -273155.0 & 20155 & 17 & 14.68 & & CT94F \\
\hline A3059-A & 025101.20 & -461857.2 & 26300 & 39 & 6.40 & & СТ94F \\
\hline A3074-B & $0258 \quad 11.34$ & -524343.2 & 21790 & 44 & 8.70 & & СТ96F \\
\hline A3077-C & 030026.88 & -512258.1 & 25511 & 29 & 7.20 & & СТ94F \\
\hline A3078-A & $0300 \quad 25.55$ & -515050.9 & 21771 & 28 & 9.74 & & СТ94F \\
\hline A3089-B & $0308 \quad 16.98$ & -364231.7 & 19524 & 33 & 11.49 & & СТ95F \\
\hline A3089-X1 & $\ldots$ & $\ldots$ & 20470 & 76 & 4.35 & & CT95F \\
\hline A3094-A & 031125.06 & -265553.5 & 20577 & 31 & 9.46 & & CT94F \\
\hline A3095-A & $03 \quad 12 \quad 26.62$ & -270825.0 & 19468 & 41 & 9.54 & & СТ95F \\
\hline A3098-A & 031343.53 & $\begin{array}{llll}-38 & 18 & 13.4\end{array}$ & 25398 & 32 & 8.08 & & СТ94F \\
\hline A3098-B & 031342.76 & -381752.0 & 23895 & 33 & 7.84 & & СТ94F \\
\hline A3100-A & $03 \quad 1352.84$ & -474734.7 & 18624 & 25 & 11.65 & & CT94F \\
\hline A3104-A & 031421.84 & -452514.0 & 21791 & 35 & 8.58 & & СТ94F \\
\hline A3106-A & 031429.91 & -580548.5 & 19523 & 38 & 9.91 & & CT96F \\
\hline A3106-C & 031433.17 & -580547.8 & 19595 & 45 & 7.32 & & СТ96F \\
\hline A3107-B & $0316 \quad 06.24$ & -424512.3 & 19833 & 26 & 11.46 & & СТ94F \\
\hline A3107-X & $\ldots$ & $\ldots$ & 19612 & 22 & 11.63 & & СТ94F \\
\hline A3109-A & 031639.42 & -435117.0 & 18608 & 32 & 9.23 & & СТ94F \\
\hline A3110-A & $\begin{array}{lll}03 & 16 & 12.40\end{array}$ & -505712.1 & 22249 & 33 & 9.04 & & CT94F \\
\hline A3111-A & $\begin{array}{lll}03 & 17 & 45.17\end{array}$ & -454520.7 & 23044 & 47 & 5.86 & & СТ94F \\
\hline A3112-A & 031757.73 & -441417.5 & 22777 & 56 & 4.48 & & CT94F \\
\hline A3112-XN & $\ldots$ & $\ldots$ & 22406 & 33 & 6.45 & & СТ94F \\
\hline A3112-XS & $\ldots$ & $\ldots$ & 22728 & 50 & 5.98 & & СТ94F \\
\hline A3120-A & 032156.48 & -511936.6 & 21413 & 44 & 8.46 & & СТ96F \\
\hline A3122-A & 032220.39 & -412146.2 & 19245 & 36 & 11.36 & & CT95F \\
\hline A3122-B & 032218.23 & -411941.5 & 20130 & 45 & 9.30 & & CT95F \\
\hline A3123-A & 032254.82 & -520149.2 & 18278 & 48 & 8.84 & & СТ96F \\
\hline A3125-B & 032754.79 & -532218.0 & 17444 & 37 & 10.18 & & СТ96F \\
\hline A3128-B & 033038.42 & -523712.2 & 17693 & 39 & 10.40 & & СТ96F \\
\hline A3133-A & 033206.05 & -455548.0 & 21377 & 67 & 4.69 & & СТ95F \\
\hline A3135-B & 033406.74 & -385934.7 & 18316 & 47 & 7.99 & & CT96F \\
\hline A3142-A & 033637.47 & -394741.1 & 20068 & 30 & 12.59 & & CT96F \\
\hline A3142-B & 033644.30 & -394804.9 & 30996 & 76 & 5.43 & & CT96F \\
\hline A3142-X & & & 20314 & 37 & 8.67 & & СТ96F \\
\hline A3144-1 & 033704.88 & -550120.8 & 13205 & 28 & 9.86 & & CT92F \\
\hline A3151-A & 034027.04 & -284037.8 & 20274 & 52 & 7.99 & & KP95F \\
\hline A3151-B & 034025.31 & -284039.2 & 20403 & 39 & 10.34 & & KP95F \\
\hline A3152-A & $0340 \quad 24.82$ & -323448.4 & 28540 & 37 & 7.05 & & CT94F \\
\hline A3152-X & 034024.11 & -323408.2 & 28528 & 36 & 5.66 & & CT94F \\
\hline A3153-A & 034103.76 & $-34 \quad 1517.2$ & 36887 & 41 & 6.42 & & СТ94F \\
\hline A3153-D & 034101.78 & -341520.2 & 36722 & 48 & 4.72 & & СТ94F \\
\hline A3154-A & 034156.83 & -320330.7 & 29042 & 38 & 9.48 & & СТ96F \\
\hline A3154-B & $0342 \quad 27.05$ & -320142.5 & 12127 & 34 & 9.53 & & СТ96F \\
\hline A3158-C & 034329.93 & -534132.6 & 18766 & 40 & 10.60 & & CT96F \\
\hline A3161-A & $0344 \quad 44.48$ & -354103.1 & 1622 & 69 & 4.03 & & CT96F \\
\hline A3164-A & 034546.96 & -570208.8 & 17823 & 47 & 9.06 & & CT96F \\
\hline A3173-B & 034920.98 & -334630.3 & 35284 & 35 & 6.30 & & СТ94F \\
\hline A3183-G & 035408.76 & -315731.3 & 21881 & 30 & 8.26 & & СТ94F \\
\hline A3188-K & 035723.66 & -270716.8 & 19316 & 22 & 11.90 & & СТ94F \\
\hline A3193-1 & 035813.54 & -521944.4 & 10103 & 31 & 8.84 & & CT92F \\
\hline A3195-A & 035854.92 & -351809.3 & 22525 & 32 & 8.34 & & СТ94F \\
\hline A3202-2 & 035929.13 & -533822.6 & 21097 & 38 & 9.84 & & CT96F \\
\hline A3223-A & 040832.63 & -304920.3 & 17923 & 29 & 11.93 & & СТ94F \\
\hline A3223-D & 040833.33 & -304838.1 & 18685 & 36 & 7.91 & & СТ94F \\
\hline A3225-A & 040905.96 & -593721.9 & 15386 & 38 & 10.06 & & СТ96F \\
\hline A3225-B & 040937.10 & -593530.7 & 16994 & 33 & 11.27 & & СТ96F \\
\hline A3229-A & 041113.93 & -624254.1 & 5075 & 29 & 8.92 & & CT94F \\
\hline A3229-B & 041039.15 & -624334.1 & 4955 & 20 & 11.72 & & CT94F \\
\hline A3231-A & 041159.58 & -643625.2 & 46480 & 46 & 8.98 & & CT96F \\
\hline A3231-B & 041115.70 & -643208.1 & 23152 & 39 & 8.88 & & СТ96F \\
\hline A3234-C & 041323.43 & -454843.8 & 37054 & 54 & 4.70 & & СТ94F \\
\hline A3241-A & 041559.66 & -644648.4 & 19600 & 26 & 11.02 & & CT94F \\
\hline A3242-A & 041751.99 & -634031.7 & 5236 & 29 & 10.19 & & CT94F \\
\hline A3242-B & $04 \quad 15 \quad 59.41$ & -633714.1 & 14377 & 20 & 12.56 & & СТ94F \\
\hline
\end{tabular}


LAUER ET AL.

Table 6 - Continued

\begin{tabular}{|c|c|c|c|c|c|c|c|}
\hline $\begin{array}{l}\text { Galaxy } \\
\text { ID }\end{array}$ & $\begin{array}{l}\text { R.A. } \\
\text { (J2000) }\end{array}$ & $\begin{array}{c}\text { Dec. } \\
\text { (J2000) }\end{array}$ & $\begin{array}{c}\mathrm{v}_{\text {helio }} \\
\mathrm{km} \mathrm{s}^{-1}\end{array}$ & $\begin{array}{c}\mathrm{V}_{e r r} \\
\mathrm{~km} \mathrm{~s}^{-1}\end{array}$ & $\begin{array}{c}\text { Tonry } \\
\mathrm{R} \text { value }\end{array}$ & $\begin{array}{c}\text { Emission } \\
\text { Flag }\end{array}$ & $\begin{array}{l}\text { Observing } \\
\text { Run ID }\end{array}$ \\
\hline A3256-A & 042750.55 & -360411.9 & 14253 & 22 & 12.19 & & СТ94F \\
\hline A3266-A & 043113.58 & -612713.1 & 18042 & 21 & 13.80 & & CT94F \\
\hline A3266-B & 043116.96 & $\begin{array}{lll}-61 & 30 & 07.2\end{array}$ & 15833 & 23 & 13.11 & & CT94F \\
\hline A3269-B & 043228.99 & -322651.9 & 15995 & 20 & 12.53 & & CT94F \\
\hline A3301-A & 050049.30 & -384028.2 & 16297 & 23 & 12.93 & & CT94F \\
\hline A3323-A & $05 \quad 1122.73$ & -285932.6 & 19226 & 49 & 8.24 & & KР95F \\
\hline A3332-A & 051635.08 & -421210.6 & 23954 & 45 & 6.45 & & СТ94F \\
\hline A3336-A & 052130.77 & -404902.2 & 23900 & 34 & 7.80 & & СТ94F \\
\hline A3341-A & 052535.27 & $\begin{array}{lll}-31 & 36 & 07.2\end{array}$ & 11024 & 30 & 9.69 & & CT94F \\
\hline A3351-B & 053203.63 & -382731.5 & 28263 & 39 & 9.51 & & CT96F \\
\hline A3351-X & $\ldots$ & $\ldots$ & 28303 & 44 & 7.44 & & CT96F \\
\hline A3358-C & 053813.98 & -203750.5 & 27452 & 56 & 8.07 & & KР95F \\
\hline A3367-1 & 054941.71 & -243243.7 & 13456 & 52 & 7.43 & & KP95F KP92F \\
\hline A3370-A & 055451.38 & $-32 \quad 1935.4$ & 27991 & 39 & 6.63 & & CT94F \\
\hline A3372-A & 055522.96 & -345134.3 & 24656 & 52 & 7.49 & & CT96F \\
\hline A3372-B & 055536.67 & -345541.8 & 24382 & 46 & 5.36 & & CT96F \\
\hline A3374-1 & 055642.97 & $-21 \quad 1512.4$ & 14237 & 62 & 5.66 & & KP92F \\
\hline A3374-1E & $\ldots$ & $\ldots$ & 64 & 41 & 7.56 & & KР95F \\
\hline A3374-1F & $\ldots$ & $\ldots$ & 78 & 29 & 11.16 & & KP95F \\
\hline A3376-1 & 060041.01 & -400246.6 & 13829 & 30 & 9.07 & & CT92F \\
\hline A3379-C & 060607.94 & $-42 \quad 17 \quad 15.9$ & 24548 & 39 & 5.74 & & CT94S \\
\hline A3380-A & 060658.19 & -492936.4 & 15692 & 34 & 11.84 & & CT96F \\
\hline A3380-C & 060652.72 & -492943.9 & 15611 & 39 & 9.70 & & CT96F \\
\hline A3381-1 & 060953.76 & -333534.1 & 11494 & 27 & 9.17 & & CT92F \\
\hline A3385-A & 061750.69 & -520114.7 & 37434 & 71 & 3.66 & & CT94S \\
\hline A3385-B & $06 \quad 17 \quad 41.04$ & -520106.8 & 36225 & 53 & 5.60 & & CT94S \\
\hline A3385-X & $\ldots$ & $\ldots$ & 36990 & 54 & 4.50 & & CT94S \\
\hline A3389-1 & 062221.30 & -645604.6 & 8248 & 33 & 7.83 & & CT92F \\
\hline A3389-2 & 062126.41 & -645937.1 & 8021 & 36 & 7.59 & & CT92F \\
\hline A3390-A & 062439.81 & -372009.4 & 9283 & 43 & 8.65 & & CT96F \\
\hline A3391-A & 062620.40 & -534136.1 & 16522 & 43 & 10.01 & & CT96F \\
\hline A3391-B & 062618.56 & -534133.6 & 16034 & 43 & 10.11 & & CT96F \\
\hline A3392-A & 062706.67 & -352916.6 & 16379 & 33 & 8.74 & & CT94F \\
\hline A3395-1 & 062736.42 & -542701.7 & 14605 & 30 & 9.78 & & СТ94F CT92F CT94S \\
\hline A3397-A & 062940.23 & -521418.5 & 22704 & 34 & 8.05 & & CT94F \\
\hline A3403-A & 064149.63 & -500015.4 & 36700 & 47 & 5.88 & & CT94F \\
\hline A3404-A & 064529.60 & -541338.0 & 48837 & 52 & 8.08 & & СТ96F \\
\hline A3407-A & 070459.29 & -490500.5 & 11780 & 48 & 6.30 & & CT94F \\
\hline A3408-A & 070829.66 & -491249.2 & 12483 & 44 & 8.76 & & CT96F \\
\hline A3408-B & 070811.40 & -490953.5 & 12610 & 40 & 8.88 & & CT96F \\
\hline A3420-A & 093206.97 & -244904.3 & 18973 & 21 & 15.08 & & CT94S \\
\hline A3428-A & 095250.72 & -334432.0 & 2601 & 23 & $\cdots$ & $\mathrm{E}$ & KP96S \\
\hline A3429-A & 095924.61 & -245815.9 & 14044 & 42 & 8.69 & & CT95S \\
\hline A3432-A & 100125.16 & -330127.5 & 20598 & 30 & 9.10 & & CT94S \\
\hline A3432-F & 100129.52 & -330238.6 & 20468 & 37 & 5.09 & & CT94S \\
\hline A3436-A & 100438.39 & -314107.6 & 2994 & 9 & $\ldots$ & $\mathrm{E}$ & CT94S CT95S \\
\hline A3455-A & 105223.35 & $-34 \quad 12 \quad 16.4$ & 28958 & 53 & 4.67 & & CT94S \\
\hline A3474-A & 111627.14 & -432503.1 & 27065 & 47 & 5.34 & & CT94S \\
\hline A3474-B & 111551.06 & -431910.7 & 28112 & 47 & 5.84 & & CT94S \\
\hline A3488-A & 114039.78 & -274532.6 & 25512 & 44 & 6.18 & & CT94S \\
\hline A3489-A & 114055.25 & -311340.8 & 30722 & 72 & 6.21 & & KP96S \\
\hline A3490-A & 114520.16 & -342601.8 & 20417 & 36 & 8.66 & & CT94S \\
\hline A3494-A & 115709.87 & $\begin{array}{lll}-32 & 09 & 07.3\end{array}$ & 20690 & 39 & 5.33 & & CT94S \\
\hline A3494-F & 115704.27 & -320854.4 & 44565 & 71 & 3.41 & & CT94S \\
\hline A3497-A & 120006.16 & -312317.3 & 20489 & 49 & 7.91 & & CT95S \\
\hline A3500-A & 120255.64 & -300739.1 & 13249 & 20 & 14.01 & & CT94S \\
\hline A3500-D & $1202 \quad 15.36$ & -301323.3 & 19837 & 36 & 4.80 & & CT95S \\
\hline A3500-H & $\begin{array}{lll}12 & 02 & 53.68\end{array}$ & -300742.5 & 13037 & 6 & $\ldots$ & $\mathrm{E}$ & CT94S \\
\hline A3501-A & 120327.19 & -283700.1 & 2022 & 98 & 3.32 & & CT95S \\
\hline A3505-A & 120842.91 & -342636.9 & 17322 & 27 & 10.78 & & CT94S \\
\hline A3505-B & 120845.97 & -342538.3 & 17546 & 25 & 11.83 & & CT94S \\
\hline A3507-A & 121256.13 & -260356.5 & 24670 & 46 & 8.40 & & CT95S \\
\hline A3507-D & 121254.85 & -260320.5 & 24755 & 56 & 6.65 & & CT95S \\
\hline A3507-F & $\begin{array}{lll}12 & 12 & 53.28\end{array}$ & -260237.1 & 24047 & 51 & 6.38 & & CT95S \\
\hline A3509-A & 121620.17 & $-33 \quad 1614.1$ & 17230 & 23 & 12.13 & & CT94S \\
\hline A3509-B & 121618.77 & -332157.3 & 25379 & 37 & 6.10 & & CT94S \\
\hline A3509-D & 121652.75 & -331537.8 & 31658 & 43 & $\cdots$ & $\mathrm{E}$ & CT94S \\
\hline A3515-H & 122300.62 & -443512.6 & 32337 & 67 & 3.61 & & CT94S \\
\hline A3524-A & 124003.75 & -341331.0 & 21792 & 39 & 7.08 & & CT94S \\
\hline A3526-1 & 124848.94 & $-41 \quad 1842.0$ & 2904 & 40 & 6.44 & & CT93S \\
\hline A3528-1 & 125422.31 & -290045.9 & 16323 & 82 & 6.75 & & KP93S \\
\hline A3530-1 & 125536.10 & -302049.3 & 16274 & 81 & 6.28 & & KP93S \\
\hline A3531-D & 125654.13 & -330014.0 & 15055 & 43 & 9.19 & & CT95S \\
\hline A3532-1 & 125722.15 & -302147.5 & 16256 & 34 & 7.47 & & CT93S \\
\hline A3535-A & 125804.34 & -282952.5 & 19885 & 49 & 7.00 & & CT95S \\
\hline A3537-1 & 130100.71 & -322628.9 & 5073 & 38 & 7.69 & & CT94S CT93S \\
\hline
\end{tabular}


Present Epoch Brightest Cluster Galaxies

Table 6 - Continued

\begin{tabular}{|c|c|c|c|c|c|c|c|}
\hline $\begin{array}{c}\text { Galaxy } \\
\text { ID }\end{array}$ & $\begin{array}{l}\text { R.A. } \\
\text { (J2000) }\end{array}$ & $\begin{array}{c}\text { Dec. } \\
\text { (J2000) }\end{array}$ & $\begin{array}{c}\mathrm{v}_{\text {helio }} \\
\mathrm{km} \mathrm{s}^{-1}\end{array}$ & $\begin{array}{c}\mathrm{v}_{\text {err }} \\
\mathrm{km} \mathrm{s}^{-1}\end{array}$ & $\begin{array}{c}\text { Tonry } \\
\mathrm{R} \text { value }\end{array}$ & $\begin{array}{c}\text { Emission } \\
\text { Flag }\end{array}$ & $\begin{array}{l}\text { Observing } \\
\text { Run ID }\end{array}$ \\
\hline A3542-1 & 130841.52 & -343431.3 & 10372 & 34 & 7.75 & & CT93S \\
\hline A3546-A & 131305.98 & -295844.1 & 32075 & 52 & 8.07 & & KP96S \\
\hline A3548-A & 131328.98 & -440424.5 & 16359 & 58 & 6.66 & & CT95S \\
\hline A3548-B & 131400.45 & -441041.6 & 15618 & 42 & 9.19 & & CT95S \\
\hline A3549-A & 131406.20 & -292529.7 & 22408 & 33 & 6.09 & & CT94S \\
\hline A3552-A & $1318 \quad 55.20$ & $\begin{array}{lll}-31 & 49 & 04.4\end{array}$ & 15573 & 25 & 11.47 & & CT94S \\
\hline A3552-F & 131853.06 & -314915.3 & 16358 & 57 & 3.64 & & CT94S \\
\hline A3552-X & & & 15954 & 73 & 3.33 & & CT94S \\
\hline A3553-1 & 131915.08 & -371046.5 & 14551 & 29 & 8.88 & & CT93S \\
\hline A3554-1 & 131931.53 & -332919.7 & 14199 & 34 & 7.87 & & CT93S \\
\hline A $3556-1$ & 132406.76 & -314015.0 & 14424 & 38 & 7.88 & & CT93S \\
\hline A3558-1 & 132756.53 & -312946.8 & 14036 & 44 & 8.71 & & CT94S KP93S \\
\hline A3559-1 & 132951.02 & -293053.1 & 14118 & 56 & 8.03 & & CT95S KP93S \\
\hline A3559-X & & $\ldots$ & 13806 & 48 & 7.05 & & CT95S \\
\hline A3560-1 & 133153.33 & -331404.4 & 3702 & 38 & 7.93 & & CT95S CT93S \\
\hline A3561-A & 133315.81 & -425018.1 & 15457 & 38 & 7.18 & & CT94S \\
\hline A3562-1 & 133334.74 & -314020.3 & 14677 & 38 & 10.52 & & CT95S \\
\hline A3563-B & 133348.86 & -423234.7 & 21202 & 31 & 9.07 & & CT94S \\
\hline A3563-D & 133348.09 & -423334.7 & 21203 & 53 & 6.18 & & CT94S \\
\hline A3563-G & 133332.91 & $-42 \quad 2651.1$ & 19133 & 33 & 7.37 & & CT94S \\
\hline A3564-1 & 133455.37 & -350557.8 & 14468 & 44 & 8.77 & & CT95S \\
\hline A3565-1 & 133639.06 & -335756.7 & 3754 & 50 & 5.29 & & CT93S \\
\hline A3567-A & 133945.50 & -362640.2 & 36638 & 67 & 5.17 & & CT94S \\
\hline A3567-D & 133937.22 & -362713.0 & 36145 & 50 & 4.52 & & CT94S \\
\hline A3569-A & 134243.71 & -354512.7 & 30191 & 54 & 7.15 & & CT95S \\
\hline A3569-B & 134242.79 & -354601.1 & 29730 & 56 & 6.40 & & CT95S \\
\hline A3570-2 & 134624.00 & -375815.5 & 11280 & 29 & 9.75 & & CT93S CT94S \\
\hline A3571-1 & 134728.42 & -325151.8 & 11563 & 44 & 6.54 & & CT93S \\
\hline A3572-1 & $1348 \quad 14.26$ & -332257.8 & 12189 & 27 & 11.20 & & CT93S CT94S \\
\hline A3574-1 & 134905.29 & -301744.9 & 4538 & 40 & 6.74 & & CT93S \\
\hline A3575-1 & 135238.36 & -325317.3 & 11127 & 43 & 7.49 & & CT95S CT93S \\
\hline A3577-A & 135414.76 & -275053.2 & 14776 & 49 & 8.65 & & CT95S \\
\hline A3577-B & 135417.84 & -275112.1 & 14082 & 39 & 8.81 & & CT95S \\
\hline A3578-B & 135733.09 & -245229.8 & 11651 & 55 & 6.41 & & KP96S \\
\hline A3581-1 & 140729.51 & -270107.0 & 6506 & 66 & 3.26 & & CT94S KP93S \\
\hline A3583-B & 141011.05 & -221834.6 & 32105 & 40 & 7.13 & & CT94S \\
\hline A3584-B & 141041.25 & -195930.0 & 42866 & 53 & 4.79 & & CT94S \\
\hline A3584-H & 141126.17 & -195712.7 & 12374 & 26 & 9.24 & & CT94S \\
\hline A3597-A & 142644.29 & -190744.2 & 31283 & 48 & 2.10 & & CT94S CT95S \\
\hline A3597-B & 142642.54 & -190749.5 & 32389 & 47 & 5.72 & & CT95S CT94S \\
\hline A3599-A & 142748.56 & -233231.5 & 7697 & 44 & 8.24 & & CT95S \\
\hline A3603-A & 143338.45 & -315116.0 & 17660 & 47 & 7.65 & & CT95S \\
\hline A3603-C & 143327.54 & -314141.1 & 17327 & 47 & 7.46 & & CT95S \\
\hline A3603-E & 143327.77 & $-3142 \quad 16.2$ & 18577 & 48 & 3.72 & & CT95S \\
\hline A3605-A & 143504.38 & -282531.9 & 20633 & 33 & 8.21 & & CT94S \\
\hline A3605-Ax & & & 20684 & 34 & 8.06 & & CT94S \\
\hline A3608-A & 144226.28 & -310013.7 & 31618 & 49 & 9.04 & & CT95S \\
\hline A3608-E & 144222.46 & -310055.7 & 32474 & 46 & 8.37 & & CT95S \\
\hline A3612-A & 145503.42 & -280322.6 & 28503 & 39 & 6.25 & & CT94S \\
\hline A3613-D & 145454.51 & -304302.3 & 28127 & 56 & 6.79 & & KP96S \\
\hline A3614-C & 145806.40 & -300613.4 & 19674 & 51 & 7.21 & & CT95S \\
\hline A3615-A & 150254.90 & -803344.2 & 20356 & 47 & 6.06 & & CT94S \\
\hline A3623-D & 153740.48 & -235612.7 & 9281 & 60 & $\ldots$ & $\mathrm{E}$ & KP96S \\
\hline A3624-A & 160123.98 & -832127.7 & 24381 & 55 & 3.71 & & CT94S \\
\hline A3624-F & $15 \quad 5703.28$ & -832912.8 & 24764 & 61 & 3.90 & & CT94S \\
\hline A3624-G & 155652.59 & -832852.6 & 26151 & 47 & 5.55 & & CT94S \\
\hline A3624-K & 155216.65 & $\begin{array}{lll}-83 & 30 & 03.3\end{array}$ & 23244 & 33 & 7.47 & & CT94S \\
\hline A3626-A & 162132.60 & -834722.3 & 20016 & 39 & 6.59 & & CT94S \\
\hline A3627-1 & 161503.68 & -605427.5 & 5385 & 43 & 6.41 & & CT93S \\
\hline A3628-A & 163129.74 & -750653.0 & 31642 & 36 & 7.03 & & CT94S \\
\hline A3630-F & $1640 \quad 05.73$ & -755941.2 & 31860 & 48 & 5.56 & & CT94S \\
\hline A3631-A & 183557.35 & -784359.4 & 21717 & 57 & 5.74 & & CT95S \\
\hline A3631-B & 183042.83 & -785033.6 & 21766 & 52 & 7.22 & & CT95S \\
\hline A3631-X & $\cdots$ & $\ldots$ & 21312 & 71 & 4.83 & & CT95S \\
\hline A3644-B & 194606.51 & $\begin{array}{lll}-80 & 02 & 10.3\end{array}$ & 38577 & 66 & 5.72 & & СТ95F \\
\hline A3651-A & 195208.62 & -550346.1 & 17923 & 28 & 11.10 & & CT94S \\
\hline A3656-1 & $2000 \quad 49.97$ & -383435.9 & 5975 & 36 & 9.07 & & СТ92F СТ94S СТ94F СТ96F \\
\hline A3667-A & 201227.34 & -564937.4 & 16621 & 51 & 8.66 & & CT95S \\
\hline A3676-A & 202424.50 & -402159.5 & 12130 & 36 & 8.01 & & CT92F CT95S \\
\hline A3677-1 & 202623.56 & -332103.5 & 13888 & 41 & 7.73 & & CT92F CT95S \\
\hline A3681-A & 202827.73 & -332540.9 & 36022 & 75 & 4.65 & & CT96F CT95S \\
\hline A3681-C & 202830.02 & -332442.3 & 36043 & 60 & 5.01 & & CT95S \\
\hline A3684-B & 203544.36 & -780506.7 & 23344 & 95 & 2.23 & & CT95F \\
\hline A3684-E & 203522.28 & -780538.0 & 23036 & 62 & 6.02 & & CT95F \\
\hline A3684-X1 & & & 51996 & 82 & 3.45 & & CT95F \\
\hline A3685-A & 203216.19 & -562539.9 & 18632 & 13 & $\ldots$ & $\mathrm{E}$ & CT95F \\
\hline
\end{tabular}


LAUER ET AL.

Table 6 - Continued

\begin{tabular}{|c|c|c|c|c|c|c|c|}
\hline $\begin{array}{c}\text { Galaxy } \\
\text { ID }\end{array}$ & $\begin{array}{l}\text { R.A. } \\
\text { (J2000) }\end{array}$ & $\begin{array}{c}\text { Dec. } \\
\text { (J2000) }\end{array}$ & $\begin{array}{c}\mathrm{v}_{\text {helio }} \\
\mathrm{km} \mathrm{s}^{-1}\end{array}$ & $\begin{array}{c}\mathrm{v}_{\text {err }} \\
\mathrm{km} \mathrm{s}^{-1}\end{array}$ & $\begin{array}{c}\text { Tonry } \\
\mathrm{R} \text { value }\end{array}$ & $\begin{array}{c}\text { Emission } \\
\text { Flag }\end{array}$ & $\begin{array}{l}\text { Observing } \\
\text { Run ID }\end{array}$ \\
\hline A3687-A & 203300.06 & -630145.2 & 22898 & 47 & 7.58 & & CT95F \\
\hline A3690-A & 203345.46 & -351259.8 & 26996 & 37 & 9.27 & & CT96F \\
\hline A3698-1 & 203556.21 & -251645.3 & 5845 & 54 & 5.96 & & CT92F KP95F \\
\hline A3703-A & 204001.74 & -612001.6 & 21551 & 44 & 8.38 & & CT96F \\
\hline A3716-1 & 205156.82 & -523747.9 & 14046 & 34 & 7.67 & & CT92F \\
\hline A3728-A & 210503.75 & -824338.8 & 29287 & 54 & 6.64 & & СТ95F \\
\hline A3728-B & 210457.03 & -824352.3 & 28919 & 70 & 5.80 & & СТ95F \\
\hline A3728-X & & & 28545 & 58 & 6.23 & & СТ95F \\
\hline A3731-A & 210141.95 & -382953.8 & 13860 & 45 & 8.72 & & CT96F \\
\hline A3733-1 & 210159.07 & -280334.5 & 11005 & 45 & 7.66 & & CT92F KP95F \\
\hline A3733-2 & 210137.58 & -280156.2 & 11794 & 35 & 7.30 & & СТ93S CT93S \\
\hline A3734-C & 210150.31 & -271952.1 & 39478 & 67 & 5.24 & & KP95F \\
\hline A3736-A & 210504.49 & $\begin{array}{lll}-43 & 25 & 09.2\end{array}$ & 14577 & 33 & 8.55 & & CT92F \\
\hline A3741-A & 211102.64 & -820854.8 & 23114 & 54 & 6.48 & & СТ96F СТ95F \\
\hline A3741-F & 211039.70 & -820945.7 & 22869 & 43 & 7.03 & & CT95F \\
\hline A3742-A & 210752.28 & $-47 \quad 1043.4$ & 4811 & 38 & 6.98 & & СТ92F \\
\hline A3744-1 & $\begin{array}{lll}21 & 07 & 16.19\end{array}$ & -252808.4 & 10994 & 49 & 7.27 & & CT95F CT92F \\
\hline A3744-2 & 210720.93 & -252915.3 & 12765 & 41 & 7.16 & & CT95F \\
\hline A $3747-1$ & 210838.93 & -432911.1 & 9318 & 38 & 7.90 & & СТ92F СТ95F \\
\hline A $3747-2$ & 210828.99 & -432924.5 & 5098 & 29 & 7.30 & & CT93S CT92F \\
\hline A3751-A & $21 \quad 14 \quad 17.42$ & -425230.1 & 9271 & 49 & 3.52 & & CT95F \\
\hline A3751-B & $2114 \quad 16.94$ & -425344.8 & 28124 & 98 & 3.14 & & CT95F \\
\hline A3752-A & 211413.64 & -270747.9 & 28298 & 47 & 8.15 & & KP95F \\
\hline A3752-B & 211429.80 & -270636.8 & 29202 & 49 & 7.50 & & KP95F \\
\hline A3753-D & 211431.97 & -264613.1 & 21840 & 57 & 5.33 & & KP95F \\
\hline A3755-A & $21 \quad 15 \quad 29.98$ & -432217.4 & 17727 & 42 & 8.35 & & СТ95F \\
\hline A3755-F & $21 \quad 1522.16$ & -432244.2 & 17875 & 11 & $\ldots$ & $\mathrm{E}$ & СТ95F \\
\hline A3756-A & 211604.66 & -424222.2 & 16308 & 40 & 10.58 & & CT96F \\
\hline A3764-A & 212617.91 & -344706.4 & 23965 & 39 & 8.27 & & СТ96F \\
\hline A3764-B & 212545.53 & -344353.7 & 22166 & 47 & 7.77 & & CT96F \\
\hline A3771-A & 212942.70 & -504926.3 & 22646 & 45 & 8.21 & & CT96F \\
\hline A3771-B & 212946.48 & -504220.8 & 22086 & 39 & 8.99 & & СТ96F \\
\hline A3771-X & & & 21939 & 36 & 10.10 & & СТ96F \\
\hline A3775-D & 213122.54 & -431336.2 & 31661 & 41 & 10.42 & & CT96F \\
\hline A3782-A & 213511.70 & -620444.3 & 16879 & 36 & 11.06 & & CT96F \\
\hline A3785-A & 213417.78 & -533813.0 & 22876 & 60 & 7.02 & & CT96F \\
\hline A3796-A & 213930.10 & -512344.6 & 22693 & 58 & 5.83 & & СТ96F \\
\hline A3799-A & 214302.43 & -723936.2 & 13714 & 54 & 6.64 & & СТ95F \\
\hline A3799-E & 214325.18 & -723950.8 & 13368 & 70 & 4.31 & & СТ95F \\
\hline A3806-A & 214622.79 & -571714.6 & 22356 & 38 & 5.97 & & CT96F \\
\hline A3806-B & 214741.05 & -571839.7 & 22840 & 52 & 6.86 & & CT96F \\
\hline A3806-Xa & $\ldots$ & $\ldots$ & 22488 & 44 & 6.85 & & CT96F \\
\hline A3806-Xb & & $\cdots$ & 22578 & 41 & 8.60 & & CT96F \\
\hline A3809-A & 214659.16 & -435357.2 & 18642 & 33 & 11.76 & & СТ96F \\
\hline A3809-B & 214750.36 & -435311.4 & 19166 & 45 & 7.07 & & СТ96F \\
\hline A3816-A & 215130.10 & -552014.9 & 11417 & 46 & 7.69 & & СТ96F \\
\hline A3822-A & 215404.33 & -575204.0 & 22615 & 59 & 6.16 & & CT96F \\
\hline A3822-C & 215500.24 & -573928.3 & 23315 & 51 & 8.00 & & CT96F \\
\hline A3825-A & 215826.38 & -602534.0 & 22528 & 46 & 7.56 & & CT96F \\
\hline A3825-C & 215903.44 & -602440.5 & 10663 & 37 & 9.12 & & СТ96F \\
\hline A3826-A & $2200 \quad 24.30$ & -561042.9 & 22654 & 48 & 7.56 & & СТ96F \\
\hline A3836-A & 220921.58 & -514827.0 & 32755 & 39 & 7.33 & & СТ94F \\
\hline A3844-A & $22 \quad 1317.70$ & -344000.6 & 21897 & 31 & 8.67 & & СТ94F \\
\hline A3849-A & 221559.17 & -513407.5 & 31948 & 53 & 4.65 & & CT94F \\
\hline A3851-A & 221700.18 & -523138.6 & 16148 & 21 & 13.13 & & CT94F \\
\hline A3867-B & 222142.67 & -573910.5 & 25100 & 38 & 9.35 & & CT96F \\
\hline A3869-A & 222031.05 & -550729.9 & 11865 & 43 & 7.72 & & СТ95F СТ92F \\
\hline A3878-A & 222651.53 & -315745.0 & 35642 & 76 & 3.39 & & CT94F \\
\hline A3878-X & & & 34837 & 80 & 2.77 & & СТ94F \\
\hline A3879-A & 222749.33 & -690124.9 & 19860 & 26 & 11.36 & & CT94F \\
\hline A3880-A & 222754.49 & -303434.5 & 17254 & 29 & 9.04 & & CT94F \\
\hline A3886-A & 223204.08 & -545431.3 & 22012 & 31 & 9.34 & & CT94F \\
\hline A3891-A & 223703.53 & -595356.4 & 25299 & 37 & 7.08 & & СТ94F \\
\hline A3895-A & 223831.60 & -364714.1 & 17517 & 38 & 9.92 & & СТ96F \\
\hline A3895-B & 223845.76 & -364439.7 & 18018 & 43 & 9.56 & & CT96F \\
\hline A3897-A & 223911.47 & -172029.7 & 22269 & 53 & 7.84 & & KP95F \\
\hline A3898-A & $22 \quad 40 \quad 10.44$ & -622454.6 & 22061 & 28 & 8.73 & & CT94F \\
\hline A3912-A & 224609.88 & -360004.5 & 20168 & 33 & 8.97 & & CT94F \\
\hline A3915-A & 224819.17 & -520312.1 & 29191 & 52 & 4.80 & & CT94F \\
\hline A3925-B & 225230.93 & -464146.1 & 15194 & 26 & 9.71 & & СТ96F \\
\hline A3959-A & 230251.94 & -332323.1 & 19691 & 21 & 11.92 & & СТ94F \\
\hline A3969-A & 230558.23 & -441059.7 & 37820 & 42 & 7.01 & & CT94F \\
\hline A3985-A & $23 \quad 16 \quad 15.11$ & -232336.1 & 31801 & 41 & 10.08 & & KP95F \\
\hline A3985-B & 231558.31 & -232017.2 & 34218 & 47 & 8.11 & & KP95F \\
\hline A3987-A & 231630.93 & -481202.7 & 36394 & 44 & 9.19 & & CT96F \\
\hline A3992-A & 232028.05 & $-73 \quad 17 \quad 17.4$ & 27040 & 42 & 8.32 & & CT96F \\
\hline
\end{tabular}


Present Epoch Brightest Cluster Galaxies

Table 6 - Continued

\begin{tabular}{|c|c|c|c|c|c|c|c|}
\hline $\begin{array}{c}\text { Galaxy } \\
\text { ID }\end{array}$ & $\begin{array}{c}\text { R.A. } \\
\text { (J2000) }\end{array}$ & $\begin{array}{c}\text { Dec. } \\
\text { (J2000) }\end{array}$ & $\begin{array}{l}\mathrm{v}_{\text {helio }} \\
\mathrm{km} \mathrm{s}^{-1}\end{array}$ & $\begin{array}{c}\mathrm{v}_{\text {err }} \\
\mathrm{km} \mathrm{s}^{-1}\end{array}$ & $\begin{array}{c}\text { Tonry } \\
\mathrm{R} \text { value }\end{array}$ & $\begin{array}{c}\text { Emission } \\
\text { Flag }\end{array}$ & $\begin{array}{c}\text { Observing } \\
\text { Run ID }\end{array}$ \\
\hline A4008-A & 233030.12 & -391652.6 & 16415 & 22 & 13.97 & & CT94F \\
\hline A4016-A & 233434.81 & $\begin{array}{ll}-69 & 1428.0\end{array}$ & 24115 & 36 & 8.72 & & CT96F \\
\hline A4026-B & 234151.37 & -372931.9 & 30647 & 42 & 6.32 & & CT94F \\
\hline A4029-A & 234347.74 & -381405.1 & 30614 & 45 & 5.45 & & CT94F \\
\hline A4038-1 & 234744.96 & $-2808 \quad 31.4$ & 8605 & 30 & 8.26 & & CT93S \\
\hline A $4038-2$ & 234728.30 & -280634.6 & 8212 & 39 & 8.44 & & СТ92F CT95F \\
\hline A4049-1 & 235136.65 & -282155.7 & 8227 & 34 & 7.71 & & CT92F \\
\hline A4053-A & 235436.09 & -273405.1 & 15005 & 23 & 13.60 & & CT94F \\
\hline A4059-1 & 235700.37 & -344532.4 & 14740 & 39 & 6.90 & & CT92F \\
\hline
\end{tabular}


Table 7

BCG Parameters

\begin{tabular}{|c|c|c|c|c|c|c|c|}
\hline Abell & $\alpha$ & $M_{m}$ & $M_{m}\left(2 r_{m}\right)$ & $M_{m}\left(4 r_{m}\right)$ & $\begin{array}{c}\sigma \\
\mathrm{km} \mathrm{s}^{-1}\end{array}$ & $\begin{array}{c}r_{x} \\
\mathrm{kpc}\end{array}$ & Ref \\
\hline 0014 & 0.983 & -22.872 & -23.564 & -24.069 & $219 \pm 15$ & 73 & $\mathrm{R}$ \\
\hline 0027 & 0.289 & -22.298 & & $\ldots$ & & $\ldots$ & $\ldots$ \\
\hline 0071 & 0.397 & -22.517 & -22.740 & & $262 \pm 14$ & & \\
\hline 0074 & 0.447 & -22.927 & -23.223 & -23.461 & $315 \pm 6$ & $\ldots$ & $\ldots$ \\
\hline 0075 & 0.455 & -22.728 & & & $295 \pm 12$ & & \\
\hline 0076 & 0.552 & -22.932 & -23.329 & & $287 \pm 2$ & 467 & $\mathrm{R}$ \\
\hline 0077 & 0.789 & -23.049 & -23.589 & -23.982 & $278 \pm 13$ & & \\
\hline 0085 & 1.039 & -23.233 & -23.848 & -24.193 & $329 \pm 17$ & 1 & $\mathrm{C}$ \\
\hline 0086 & 0.676 & -23.209 & -23.648 & -23.944 & $274 \pm 15$ & 215 & $\mathrm{R}$ \\
\hline 0102 & 0.539 & -23.051 & -23.435 & -23.717 & $271 \pm 11$ & & \\
\hline 0114 & 0.520 & -23.076 & -23.427 & -23.702 & $299 \pm 13$ & & \\
\hline 0116 & 0.470 & -22.948 & -23.236 & -23.447 & $327 \pm 11$ & 76 & $\mathrm{R}$ \\
\hline 0117 & 0.670 & -23.224 & -23.659 & -23.963 & $323 \pm 11$ & 177 & $\mathrm{R}$ \\
\hline 0119 & 0.767 & -23.046 & -23.578 & -23.909 & $283 \pm 13$ & 23 & $\mathrm{C}$ \\
\hline 0133 & 0.775 & -22.987 & -23.507 & -23.888 & $273 \pm 13$ & 5 & $\mathrm{C}$ \\
\hline 0134 & 0.357 & -22.486 & -22.708 & $\ldots$ & $188 \pm 24$ & 1023 & $\mathrm{R}$ \\
\hline 0147 & 0.425 & -22.819 & -23.087 & & $289 \pm 3$ & 450 & $\mathrm{R}$ \\
\hline 0150 & 0.678 & -22.921 & -23.385 & -23.743 & $258 \pm 18$ & 34 & $\mathrm{R}$ \\
\hline 0151 & 0.712 & -23.138 & -23.624 & -24.035 & $308 \pm 12$ & 49 & $\mathrm{R}$ \\
\hline 0152 & 0.796 & -22.719 & -23.266 & -23.659 & $239 \pm 15$ & & \\
\hline 0154 & 0.601 & -23.225 & -23.644 & -24.018 & $314 \pm 12$ & 95 & $\mathrm{R}$ \\
\hline 0158 & 0.656 & -22.705 & -23.115 & & $262 \pm 16$ & & \\
\hline 0161 & 0.622 & -22.596 & -23.034 & -23.463 & $228 \pm 17$ & & \\
\hline 0168 & 0.571 & -22.848 & -23.226 & & $253 \pm 1$ & 286 & $\mathrm{R}$ \\
\hline 0171 & 0.561 & -23.192 & -23.569 & -23.874 & $337 \pm 10$ & $\ldots$ & \\
\hline 0174 & 0.596 & -22.622 & -23.022 & $\ldots$ & $290 \pm 15$ & $\ldots$ & $\ldots$ \\
\hline 0179 & 0.420 & -22.420 & -22.694 & & $269 \pm 14$ & & \\
\hline 0189 & 0.322 & -22.109 & & & $212 \pm 10$ & .. & \\
\hline 0193 & 0.690 & -22.944 & -23.448 & & $462 \pm 39$ & 1 & C \\
\hline 0194 & 0.587 & -22.611 & -22.981 & .. & $262 \pm 6$ & 57 & $\mathrm{R}$ \\
\hline 0195 & 0.410 & -22.706 & -22.985 & & $275 \pm 3$ & $\ldots$ & \\
\hline 0208 & 0.625 & -22.989 & -23.439 & -23.811 & $319 \pm 9$ & $\ldots$ & \\
\hline 0225 & 0.708 & -22.965 & -23.439 & -23.796 & $271 \pm 12$ & $\ldots$ & $\ldots$ \\
\hline 0240 & 0.805 & -22.788 & -23.306 & & $240 \pm 20$ & & \\
\hline 0245 & 0.427 & -22.401 & -22.628 & 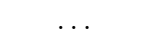 & $202 \pm 28$ & & \\
\hline 0246 & 0.525 & -22.556 & -22.900 & . & $316 \pm 16$ & 37 & $\mathrm{R}$ \\
\hline 0257 & 0.571 & -22.816 & -23.164 & & $257 \pm 18$ & & \\
\hline 0260 & 0.574 & -22.973 & -23.388 & 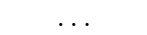 & $298 \pm 10$ & $\ldots$ & $\ldots$ \\
\hline 0261 & 0.564 & -22.897 & -23.283 & & $246 \pm 17$ & & \\
\hline 0262 & 0.734 & -22.383 & -22.856 & & $232 \pm 7$ & 2 & $\mathrm{C}$ \\
\hline 0267 & 0.732 & -22.326 & -22.827 & & $206 \pm 22$ & 576 & $\mathrm{C}$ \\
\hline 0268 & 0.424 & -22.087 & -22.365 & & $192 \pm 26$ & & \\
\hline 0279 & 0.718 & -23.162 & -23.662 & -24.080 & $268 \pm 12$ & 206 & $\mathrm{R}$ \\
\hline 0292 & 0.802 & -23.074 & -23.589 & -23.971 & $289 \pm 16$ & $\ldots$ & \\
\hline 0295 & 0.539 & -22.789 & -23.185 & & $258 \pm 12$ & $\ldots$ & $\ldots$ \\
\hline 0311 & 0.742 & -23.213 & -23.726 & -24.149 & $248 \pm 19$ & $\ldots$ & \\
\hline 0326 & 0.353 & -22.183 & -22.465 & $\ldots$ & $336 \pm 0$ & $\ldots$ & $\ldots$ \\
\hline 0347 & 0.577 & -22.524 & -22.935 & & $269 \pm 16$ & $\ldots$ & \\
\hline 0357 & 0.291 & -22.060 & & & $225 \pm 19$ & .. & \\
\hline 0358 & 0.669 & -22.894 & -23.317 & -23.636 & $260 \pm 14$ & 96 & $\mathrm{R}$ \\
\hline 0376 & 0.682 & -22.861 & -23.326 & -23.686 & $265 \pm 22$ & 75 & $\mathrm{R}$ \\
\hline 0386 & 0.335 & -22.555 & -22.738 & & $276 \pm 9$ & $\ldots$ & \\
\hline 0397 & 0.552 & -22.882 & -23.286 & -23.572 & $314 \pm 3$ & & \\
\hline 0399 & 0.962 & -23.236 & -23.863 & -24.378 & $242 \pm 24$ & 26 & $\mathrm{C}$ \\
\hline 0400 & 0.677 & -22.335 & -22.899 & & & 5 & $\mathrm{C}$ \\
\hline 0401 & 0.929 & -23.344 & -23.985 & -24.490 & $336 \pm 18$ & 62 & $\mathrm{C}$ \\
\hline 0404 & 0.539 & -22.845 & -23.192 & & $264 \pm 14$ & $\cdots$ & $\ldots$ \\
\hline 0407 & 0.778 & -22.819 & -23.406 & -23.898 & & & \\
\hline 0415 & 0.851 & -22.952 & -23.562 & -24.040 & $253 \pm 21$ & 62 & $\mathrm{R}$ \\
\hline 0423 & 0.609 & -22.792 & -23.203 & -23.511 & $294 \pm 11$ & & \\
\hline 0428 & 0.286 & -22.325 & -22.481 & & $230 \pm 17$ & .. & \\
\hline 0436 & 0.622 & -22.958 & -23.388 & -23.714 & $326 \pm 15$ & .. & \\
\hline 0450 & 0.405 & -22.638 & -22.899 & & $301 \pm 11$ & & \\
\hline 0496 & 0.766 & -23.198 & -23.730 & -24.152 & $290 \pm 4$ & 3 & $\mathrm{C}$ \\
\hline 0498 & 0.620 & -23.300 & -23.724 & -24.019 & $278 \pm 23$ & & \\
\hline 0500 & 0.610 & -23.114 & -23.556 & -23.957 & $365 \pm 10$ & 36 & $\mathrm{R}$ \\
\hline 0505 & 0.735 & -23.609 & -24.108 & & $383 \pm 14$ & & \\
\hline 0514 & 0.517 & -23.137 & -23.461 & -23.671 & $358 \pm 10$ & 1060 & $\mathrm{R}$ \\
\hline 0533 & 0.487 & -22.855 & -23.177 & -23.590 & $282 \pm 6$ & & \\
\hline 0539 & 0.457 & -22.800 & -23.153 & & $282 \pm 8$ & 281 & C \\
\hline 0548 & 0.474 & -22.936 & -23.256 & -23.630 & $222 \pm 18$ & 542 & $\mathrm{R}$ \\
\hline 0564 & 0.338 & -22.709 & & & $182 \pm 16$ & & \\
\hline 0568 & 0.557 & -23.200 & -23.613 & 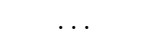 & $299 \pm 35$ & . & \\
\hline 0569 & 0.473 & -22.708 & -23.003 & $\ldots$ & $245 \pm 3$ & & \\
\hline 0576 & 0.298 & -22.477 & -22.619 & & $278 \pm 17$ & 387 & $\mathrm{C}$ \\
\hline
\end{tabular}


Present Epoch Brightest Cluster Galaxies

Table 7 - Continued

\begin{tabular}{|c|c|c|c|c|c|c|c|}
\hline Abell & $\alpha$ & $M_{m}$ & $M_{m}\left(2 r_{m}\right)$ & $M_{m}\left(4 r_{m}\right)$ & $\begin{array}{c}\sigma \\
\mathrm{km} \mathrm{s}^{-1}\end{array}$ & $\begin{array}{c}r_{x} \\
\mathrm{kpc}\end{array}$ & Ref \\
\hline 0582 & 0.484 & -22.819 & -23.163 & & $288 \pm 15$ & & \\
\hline 0592 & 0.461 & -22.948 & -23.231 & .. & $342 \pm 10$ & & \\
\hline 0595 & 0.402 & -22.789 & -23.032 & & $258 \pm 20$ & 816 & $\mathrm{R}$ \\
\hline 0600 & 0.648 & -22.725 & -23.126 & -23.497 & $191 \pm 24$ & & \\
\hline 0602 & 0.282 & -22.385 & -22.548 & s & & 1134 & $\mathrm{R}$ \\
\hline 0634 & 0.485 & -22.603 & -22.928 & & $251 \pm 9$ & & $\ldots$ \\
\hline 0644 & 0.796 & -22.954 & -23.549 & -24.012 & $262 \pm 15$ & 11 & $\mathrm{C}$ \\
\hline 0671 & 0.710 & -23.306 & -23.821 & -24.232 & & 111 & $\mathrm{R}$ \\
\hline 0690 & 0.633 & -23.427 & -23.882 & -24.260 & & $\ldots$ & $\ldots$ \\
\hline 0695 & 0.596 & -23.048 & -23.412 & & $363 \pm 14$ & & 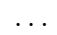 \\
\hline 0744 & 0.662 & -23.034 & -23.473 & -23.813 & & 2 & $\mathrm{C}$ \\
\hline 0754 & 0.748 & -23.358 & -23.879 & $\ldots$ & & 750 & $\mathrm{C}$ \\
\hline 0757 & 0.428 & -22.423 & -22.727 & $\ldots$ & $250 \pm 9$ & & \\
\hline 0779 & 0.593 & -23.255 & -23.685 & & $327 \pm 8$ & 26 & $\mathrm{R}$ \\
\hline 0780 & 0.794 & -23.026 & -23.532 & -23.881 & $266 \pm 7$ & 20 & $\mathrm{R}$ \\
\hline 0819 & 0.586 & -23.146 & -23.543 & -23.791 & & $\ldots$ & $\ldots$ \\
\hline 0834 & 0.696 & -22.768 & -23.239 & & $254 \pm 24$ & $\ldots$ & \\
\hline 0838 & 0.596 & -22.638 & -23.059 & & $226 \pm 6$ & $\ldots$ & $\ldots$ \\
\hline 0841 & 0.606 & -23.114 & -23.548 & -23.897 & $273 \pm 10$ & & \\
\hline 0912 & 0.440 & -22.390 & -22.663 & -23.006 & $207 \pm 15$ & & \\
\hline 0957 & 0.759 & -23.297 & -23.840 & -24.287 & $332 \pm 0$ & 169 & $\mathrm{R}$ \\
\hline 0970 & 0.559 & -22.388 & -22.830 & & & 120 & $\mathrm{R}$ \\
\hline 0978 & 0.615 & -23.135 & -23.594 & -23.965 & $248 \pm 4$ & 23 & $\mathrm{R}$ \\
\hline 0979 & 0.605 & -22.869 & -23.271 & -23.561 & $284 \pm 5$ & & \\
\hline 0993 & 0.552 & -23.221 & -23.570 & -23.800 & $294 \pm 15$ & 25 & $\mathrm{R}$ \\
\hline 0999 & 0.441 & -22.689 & -23.017 & $\ldots$ & $241 \pm 31$ & $-\infty$ & - \\
\hline 1003 & 0.644 & -22.748 & -23.169 & 年 & $324 \pm 10$ & $\ldots$ & 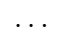 \\
\hline 1016 & 0.433 & -22.497 & -22.799 & .. & $255 \pm 2$ & & \\
\hline 1020 & 0.448 & -22.924 & -23.231 & 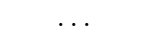 & $302 \pm 7$ & 481 & $\mathrm{R}$ \\
\hline 1032 & 0.391 & -22.768 & -23.007 & & $269 \pm 16$ & 543 & $\mathrm{R}$ \\
\hline 1035 & 0.600 & -23.154 & -23.542 & -23.824 & & 128 & $\mathrm{R}$ \\
\hline 1060 & 0.846 & -22.764 & -23.346 & & $208 \pm 12$ & 0 & $\mathrm{C}$ \\
\hline 1066 & 0.568 & -22.823 & -23.244 & -23.563 & $308 \pm 6$ & & \\
\hline 1069 & 0.757 & -23.130 & -23.696 & -24.050 & $\ldots$ & 110 & $\mathrm{R}$ \\
\hline 1090 & 0.518 & -22.706 & -23.078 & $\ldots$ & . & $\ldots$ & $\ldots$ \\
\hline 1100 & 0.562 & -22.803 & -23.184 & $\ldots$ & $246 \pm 32$ & & \\
\hline 1139 & 0.583 & -22.744 & -23.162 & .. & $263 \pm 20$ & 197 & $\mathrm{R}$ \\
\hline 1142 & 0.543 & -22.768 & -23.189 & $\ldots$ & $297 \pm 7$ & 52 & $\mathrm{R}$ \\
\hline 1145 & 0.364 & -22.254 & -22.478 & .. & & $\ldots$ & \\
\hline 1149 & 0.609 & -22.961 & -23.346 & $\ldots$ & $283 \pm 12$ & $\ldots$ & $\ldots$ \\
\hline 1155 & 0.504 & -22.664 & -23.016 & & & & \\
\hline 1169 & 0.407 & -22.437 & -22.717 & . & $269 \pm 23$ & 143 & $\mathrm{R}$ \\
\hline 1171 & 0.307 & -22.636 & -22.814 & & $258 \pm 15$ & 578 & $\mathrm{R}$ \\
\hline 1177 & 0.726 & -22.888 & -23.391 & -23.826 & $231 \pm 13$ & & \\
\hline 1185 & 0.621 & -22.882 & -23.270 & $\ldots$ & $\ldots$ & 161 & $\mathrm{R}$ \\
\hline 1186 & 0.522 & -22.306 & -22.672 & & & & \\
\hline 1187 & 0.599 & -23.077 & -23.479 & -23.764 & & 428 & $\mathrm{R}$ \\
\hline 1190 & 0.802 & -22.935 & -23.488 & -23.875 & $230 \pm 23$ & $\ldots$ & $\ldots$ \\
\hline 1203 & 0.582 & -23.078 & -23.485 & -23.800 & $280 \pm 15$ & $\ldots$ & $\ldots$ \\
\hline 1213 & 0.516 & -22.884 & -23.185 & $\ldots$ & $281 \pm 19$ & $\ldots$ & $\ldots$ \\
\hline 1216 & 0.452 & -22.633 & -22.921 & $\ldots$ & $\ldots$ & & \\
\hline 1228 & 0.428 & -22.490 & -22.784 & & & 183 & $\mathrm{R}$ \\
\hline 1238 & 0.703 & -22.819 & -23.332 & -23.799 & & 130 & $\mathrm{R}$ \\
\hline 1257 & 0.248 & -22.233 & & $\ldots$ & $260 \pm 8$ & $\ldots$ & $\ldots$ \\
\hline 1267 & 0.334 & -22.546 & -22.747 & & $257 \pm 7$ & & \\
\hline 1279 & 0.542 & -22.636 & -22.997 & .. & $236 \pm 3$ & & \\
\hline 1291 & 0.378 & -22.549 & -22.752 & & & 298 & $\mathrm{R}$ \\
\hline 1308 & 0.550 & -23.207 & -23.613 & -23.918 & $282 \pm 12$ & & \\
\hline 1314 & 0.544 & -22.888 & -23.315 & -23.718 & $291 \pm 23$ & 71 & $\mathrm{R}$ \\
\hline 1317 & 0.645 & -23.036 & -23.547 & -24.050 & $313 \pm 13$ & 87 & $\mathrm{R}$ \\
\hline 1318 & 0.477 & -22.860 & -23.169 & $\ldots$ & $293 \pm 12$ & 1458 & $\mathrm{R}$ \\
\hline 1334 & 0.419 & -22.701 & -22.980 & & $265 \pm 4$ & & \\
\hline 1344 & 0.672 & -23.190 & -23.645 & -24.041 & $302 \pm 0$ & & \\
\hline 1365 & 0.443 & -22.812 & -23.107 & $-2.0=$ & $283 \pm 11$ & 91 & $\mathrm{R}$ \\
\hline 1367 & 0.547 & -23.036 & -23.384 & & $308 \pm 3$ & 354 & $\mathrm{R}$ \\
\hline 1371 & 0.611 & -22.827 & -23.250 & -23.663 & $260 \pm 16$ & 198 & $\mathrm{R}$ \\
\hline 1375 & 0.517 & -23.344 & -23.666 & -23.850 & $296 \pm 5$ & & \\
\hline 1377 & 0.466 & -22.759 & -23.083 & -23.339 & $294 \pm 11$ & 140 & $\mathrm{R}$ \\
\hline 1383 & 0.377 & -22.560 & -22.785 & $\ldots$ & & 947 & $\mathrm{R}$ \\
\hline 1400 & 0.715 & -22.536 & -22.981 & & & 190 & $\mathrm{R}$ \\
\hline 1404 & 0.463 & -22.721 & -23.015 & -23.267 & $232 \pm 5$ & & \\
\hline 1424 & 0.565 & -23.259 & -23.631 & $\ldots$ & $313 \pm 4$ & 168 & $\mathrm{R}$ \\
\hline 1436 & 0.329 & -22.638 & -22.837 & $\cdots$ & $302 \pm 12$ & 968 & $\mathrm{R}$ \\
\hline 1452 & 0.501 & -22.901 & -23.232 & & $327 \pm 12$ & & \\
\hline 1461 & 0.470 & -22.580 & -22.869 & $\cdots$ & $250 \pm 22$ & $\cdots$ & $\ldots$ \\
\hline 1474 & 0.530 & -22.347 & -22.675 & $\ldots$ & $\ldots$ & $\ldots$ & $\ldots$ \\
\hline
\end{tabular}


LAUER ET AL.

Table 7 - Continued

\begin{tabular}{|c|c|c|c|c|c|c|c|}
\hline Abell & $\alpha$ & $M_{m}$ & $M_{m}\left(2 r_{m}\right)$ & $M_{m}\left(4 r_{m}\right)$ & $\begin{array}{c}\sigma \\
\mathrm{km} \mathrm{s}^{-1}\end{array}$ & $\begin{array}{c}r_{x} \\
\mathrm{kpc}\end{array}$ & Ref \\
\hline 1507 & 0.640 & -22.887 & -23.305 & & & 139 & $\mathrm{R}$ \\
\hline 1520 & 0.704 & -23.405 & -23.899 & -24.332 & $290 \pm 13$ & & \\
\hline 1534 & 0.569 & -23.095 & -23.489 & -23.801 & $299 \pm 7$ & 157 & $\mathrm{R}$ \\
\hline 1569 & 0.719 & -23.318 & -23.776 & -24.088 & $314 \pm 12$ & 5 & $\mathrm{C}$ \\
\hline 1589 & 0.660 & -23.430 & -23.888 & -24.293 & $\ldots$ & $\ldots$ & $\ldots$ \\
\hline 1610 & 0.476 & -23.242 & -23.574 & -23.807 & & & \\
\hline 1630 & 0.617 & -23.058 & -23.469 & -23.799 & $316 \pm 11$ & 157 & $\mathrm{R}$ \\
\hline 1631 & 0.665 & -23.083 & -23.519 & -23.884 & $254 \pm 23$ & & \\
\hline 1644 & 1.003 & -23.184 & -23.847 & -24.336 & $212 \pm 34$ & 2 & $\mathrm{C}$ \\
\hline 1648 & 0.483 & -23.004 & -23.402 & -23.713 & $296 \pm 8$ & & \\
\hline 1656 & 0.597 & -23.417 & -23.823 & & $386 \pm 2$ & 256 & $\mathrm{R}$ \\
\hline 1691 & 0.799 & -23.236 & -23.752 & -24.132 & $257 \pm 3$ & 45 & $\mathrm{R}$ \\
\hline 1709 & 0.536 & -23.032 & -23.364 & -23.683 & $283 \pm 5$ & & \\
\hline 1736 & 0.591 & -23.503 & -23.897 & -24.248 & $303 \pm 11$ & 687 & $\mathrm{C}$ \\
\hline 1741 & 0.417 & -22.784 & -23.066 & & $265 \pm 14$ & & \\
\hline 1749 & 0.557 & -23.168 & -23.569 & -23.894 & $302 \pm 7$ & 28 & $\mathrm{R}$ \\
\hline 1767 & 0.790 & -23.216 & -23.768 & -24.149 & & 25 & $\mathrm{R}$ \\
\hline 1773 & 0.585 & -22.972 & -23.391 & -23.834 & $383 \pm 21$ & 72 & $\mathrm{R}$ \\
\hline 1775 & 0.715 & -23.208 & -23.706 & -24.080 & $285 \pm 20$ & 48 & $\mathrm{R}$ \\
\hline 1780 & 0.469 & -22.913 & -23.210 & -23.257 & $259 \pm 12$ & 461 & $\mathrm{R}$ \\
\hline 1795 & 0.783 & -22.984 & -23.556 & -24.030 & & 12 & $\mathrm{C}$ \\
\hline 1800 & 0.940 & -23.315 & -23.924 & -24.350 & $324 \pm 17$ & 83 & $\mathrm{R}$ \\
\hline 1809 & 0.610 & -23.432 & -23.864 & -24.265 & & 47 & $\mathrm{R}$ \\
\hline 1825 & 0.565 & -22.848 & -23.238 & -23.530 & & . & \\
\hline 1827 & 0.463 & -22.917 & -23.205 & $\ldots$ & $300 \pm 11$ & $\ldots$ & \\
\hline 1828 & 0.342 & -22.628 & -22.845 & & & & \\
\hline 1831 & 0.792 & -23.337 & -23.886 & -24.318 & $331 \pm 12$ & 51 & $\mathrm{R}$ \\
\hline 1836 & 0.603 & -23.160 & -23.539 & -23.811 & $286 \pm 16$ & & \\
\hline 1873 & 0.451 & -22.287 & -22.599 & & $317 \pm 13$ & 79 & $\mathrm{R}$ \\
\hline 1890 & 0.674 & -23.382 & -23.838 & -24.164 & $335 \pm 4$ & 236 & $\mathrm{R}$ \\
\hline 1898 & 0.623 & -22.820 & -23.245 & -23.564 & & 1155 & $\mathrm{R}$ \\
\hline 1899 & 0.481 & -22.698 & -22.986 & -23.168 & $262 \pm 13$ & & \\
\hline 1904 & 0.682 & -23.262 & -23.729 & -24.069 & & 289 & $\mathrm{R}$ \\
\hline 1913 & 0.459 & -22.928 & -23.223 & & $248 \pm 15$ & 896 & $\mathrm{R}$ \\
\hline 1964 & 0.560 & -22.788 & -23.225 & -23.647 & $313 \pm 12$ & & \\
\hline 1982 & 0.644 & -22.823 & -23.249 & -23.541 & & & \\
\hline 1983 & 0.367 & -22.736 & -22.953 & & $304 \pm 6$ & 653 & $\mathrm{R}$ \\
\hline 1991 & 0.822 & -22.967 & -23.537 & -23.937 & $269 \pm 5$ & 11 & $\mathrm{C}$ \\
\hline 2022 & 0.644 & -23.035 & -23.504 & -23.934 & $\ldots$ & 61 & $\mathrm{R}$ \\
\hline 2028 & 0.655 & -23.221 & -23.663 & -24.028 & & & \\
\hline 2029 & 0.935 & -23.731 & -24.385 & -24.924 & $386 \pm 2$ & 1 & C \\
\hline 2040 & 0.678 & -22.542 & -23.113 & -23.556 & $246 \pm 15$ & 39 & $\mathrm{R}$ \\
\hline 2052 & 0.855 & -22.946 & -23.534 & -23.991 & $203 \pm 20$ & 0 & $\mathrm{C}$ \\
\hline 2061 & 0.554 & -23.383 & -23.775 & -24.147 & & 176 & $\mathrm{R}$ \\
\hline 2063 & 0.776 & -22.753 & -23.248 & -23.576 & $198 \pm 19$ & 11 & $\mathrm{C}$ \\
\hline 2065 & 0.623 & -22.737 & -23.149 & $\ldots$ & $265 \pm 1$ & 105 & $\mathrm{C}$ \\
\hline 2067 & 0.759 & -22.552 & -23.059 & & & 40 & $\mathrm{R}$ \\
\hline 2079 & 0.775 & -23.247 & -23.807 & -24.410 & $289 \pm 6$ & 158 & $\mathrm{R}$ \\
\hline 2089 & 0.582 & -23.173 & -23.584 & -23.943 & $330 \pm 7$ & & \\
\hline 2092 & 0.631 & -22.380 & -22.833 & & & 132 & $\mathrm{R}$ \\
\hline 2107 & 0.741 & -23.231 & -23.736 & -24.117 & $308 \pm 12$ & 1 & $\mathrm{C}$ \\
\hline 2147 & 0.694 & -22.850 & -23.303 & -23.734 & $280 \pm 10$ & 0 & $\mathrm{C}$ \\
\hline 2151 & 0.750 & -22.813 & -23.318 & -23.634 & $299 \pm 5$ & 1 & $\mathrm{C}$ \\
\hline 2152 & 0.412 & -22.786 & -23.037 & -23.154 & $282 \pm 10$ & 63 & $\mathrm{R}$ \\
\hline 2162 & 0.493 & -22.996 & -23.349 & 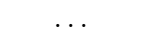 & $303 \pm 17$ & 49 & $\mathrm{R}$ \\
\hline 2184 & 0.353 & -22.253 & -22.457 & & $247 \pm 8$ & & \\
\hline 2197 & 0.574 & -23.356 & -23.742 & -24.034 & $276 \pm 2$ & 886 & $\mathrm{R}$ \\
\hline 2198 & 0.292 & -22.002 & -22.136 & & $209 \pm 20$ & & \\
\hline 2199 & 0.760 & -23.135 & -23.646 & -24.053 & $297 \pm 5$ & 1 & $\mathrm{C}$ \\
\hline 2241 & 0.574 & -22.495 & -22.838 & $\ldots$ & & & \\
\hline 2247 & 0.367 & -22.590 & -22.819 & 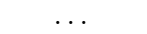 & $257 \pm 14$ & & \\
\hline 2248 & 0.469 & -22.747 & -23.054 & & & 424 & $\mathrm{R}$ \\
\hline 2250 & 0.544 & -22.699 & -23.121 & -23.560 & $297 \pm 15$ & & \\
\hline 2256 & 0.737 & -23.205 & -23.675 & -24.000 & & 143 & $\mathrm{C}$ \\
\hline 2271 & 0.783 & -23.042 & -23.576 & -23.942 & $289 \pm 14$ & 17 & $\mathrm{R}$ \\
\hline 2293 & 0.510 & -22.722 & -23.070 & & $222 \pm 17$ & & \\
\hline 2308 & 0.657 & -22.962 & -23.390 & -23.693 & $269 \pm 15$ & & \\
\hline 2309 & 0.571 & -22.817 & -23.203 & $\ldots$ & $298 \pm 13$ & $\ldots$ & . \\
\hline 2325 & 0.517 & -22.760 & -23.055 & & $253 \pm 10$ & & \\
\hline 2331 & 0.810 & -22.803 & -23.349 & -23.798 & $253 \pm 16$ & 246 & $\mathrm{R}$ \\
\hline 2361 & 0.368 & -22.836 & -23.088 & $\ldots$ & & & \\
\hline 2362 & 0.480 & -23.090 & -23.416 & & $307 \pm$ & & \\
\hline 2366 & 0.554 & -23.095 & -23.501 & $\cdots$ & $279 \pm 10$ & 175 & $\mathrm{R}$ \\
\hline 2370 & 0.257 & -21.729 & & & $240 \pm 22$ & & \\
\hline 2372 & 0.497 & -22.920 & -23.245 & -23.508 & $267 \pm 13$ & $\cdots$ & \\
\hline 2382 & 0.794 & -22.854 & -23.402 & & $243 \pm 13$ & 93 & $\mathrm{R}$ \\
\hline
\end{tabular}


Present Epoch Brightest Cluster Galaxies

Table 7 - Continued

\begin{tabular}{|c|c|c|c|c|c|c|c|}
\hline Abell & $\alpha$ & $M_{m}$ & $M_{m}\left(2 r_{m}\right)$ & $M_{m}\left(4 r_{m}\right)$ & $\begin{array}{c}\sigma \\
\mathrm{km} \mathrm{s}^{-1}\end{array}$ & $\begin{array}{c}r_{x} \\
\mathrm{kpc}\end{array}$ & Ref \\
\hline 2383 & 0.271 & -22.081 & -22.257 & & & & \\
\hline 2388 & 0.581 & -22.515 & -22.910 & . & $215 \pm 27$ & & \\
\hline 2399 & 0.516 & -22.832 & -23.154 & $\ldots$ & $276 \pm 19$ & 193 & $\mathrm{R}$ \\
\hline 2401 & 0.574 & -23.079 & -23.457 & $\cdots$ & $304 \pm 12$ & 58 & $\mathrm{R}$ \\
\hline 2405 & 0.330 & -22.416 & -22.645 & $\ldots$ & $254 \pm 11$ & & \\
\hline 2412 & 0.422 & -22.730 & -23.111 & & $269 \pm 13$ & 314 & $\mathrm{R}$ \\
\hline 2415 & 0.543 & -22.934 & -23.277 & -23.578 & $283 \pm 9$ & 600 & $\mathrm{R}$ \\
\hline 2457 & 0.617 & -23.226 & -23.641 & -23.977 & $327 \pm 14$ & 21 & $\mathrm{R}$ \\
\hline 2459 & 0.397 & -22.817 & -23.051 & & $276 \pm 13$ & & \\
\hline 2462 & 0.567 & -23.133 & -23.556 & -23.932 & $349 \pm 9$ & 1 & $\mathrm{C}$ \\
\hline 2469 & 0.428 & -22.436 & -22.669 & & $221 \pm 20$ & & \\
\hline 2480 & 0.472 & -23.176 & -23.488 & -23.752 & $305 \pm 13$ & & \\
\hline 2492 & 0.569 & -22.891 & -23.267 & & $287 \pm 11$ & 125 & $\mathrm{R}$ \\
\hline 2495 & 0.920 & -22.833 & -23.510 & -24.054 & $258 \pm 15$ & & \\
\hline 2511 & 0.377 & -22.499 & -22.723 & & $258 \pm 19$ & $\ldots$ & $\ldots$ \\
\hline 2524 & 0.595 & -23.103 & -23.489 & -23.823 & & & \\
\hline 2525 & 0.535 & -22.579 & -22.945 & & $301 \pm 11$ & $\ldots$ & $\ldots$ \\
\hline 2558 & 0.546 & -22.554 & -22.942 & & $270 \pm 14$ & $\because$ & \\
\hline 2559 & 0.801 & -23.070 & -23.603 & -23.984 & $276 \pm 16$ & & \\
\hline 2572 & 0.540 & -22.785 & -23.238 & & $323 \pm 8$ & 57 & $\mathrm{R}$ \\
\hline 2589 & 0.755 & -22.886 & -23.403 & -23.8 & $289 \pm 9$ & 3 & $\mathrm{C}$ \\
\hline 2593 & 0.795 & -22.828 & -23.315 & & $248 \pm 7$ & 10 & $\mathrm{R}$ \\
\hline 2618 & 0.648 & -23.330 & -23.772 & -24.137 & $304 \pm 11$ & & \\
\hline 2622 & 0.640 & -22.956 & -23.369 & -23.624 & $295 \pm 16$ & 55 & $\mathrm{R}$ \\
\hline 2625 & 0.452 & -22.963 & -23.263 & & $269 \pm 15$ & 2065 & $\mathrm{R}$ \\
\hline 2626 & 0.856 & -22.971 & -23.501 & -23.784 & $278 \pm 18$ & 2 & $\mathrm{C}$ \\
\hline 2630 & 0.489 & -22.965 & -23.260 & & $273 \pm 20$ & & \\
\hline 2634 & 0.627 & -23.097 & -23.553 & -23.869 & $366 \pm 8$ & 24 & $\mathrm{R}$ \\
\hline 2637 & 0.494 & -23.199 & -23.512 & -23.768 & $285 \pm 15$ & 177 & $\mathrm{R}$ \\
\hline 2644 & 0.500 & -22.756 & -23.070 & -23.283 & $303 \pm 12$ & 78 & $\mathrm{R}$ \\
\hline 2656 & 0.772 & -22.861 & -23.344 & -23.627 & $286 \pm 12$ & & \\
\hline 2657 & 0.336 & -22.408 & -22.605 & 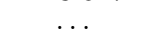 & $206 \pm 16$ & 376 & C \\
\hline 2660 & 0.553 & -22.649 & -23.021 & & $225 \pm 17$ & 154 & $\mathrm{R}$ \\
\hline 2665 & 0.804 & -23.137 & -23.669 & -24.100 & $285 \pm 24$ & & \\
\hline 2666 & 0.547 & -23.054 & -23.389 & -23.726 & $292 \pm 8$ & 65 & $\mathrm{R}$ \\
\hline 2670 & 0.686 & -23.342 & -23.848 & -24.250 & $299 \pm 7$ & 68 & $\mathrm{R}$ \\
\hline 2675 & 0.572 & -22.889 & -23.263 & -23.555 & $256 \pm 22$ & 176 & $\mathrm{R}$ \\
\hline 2678 & 0.487 & -22.682 & -22.982 & & $285 \pm 18$ & $\ldots$ & \\
\hline 2716 & 0.323 & -22.786 & -22.995 & -23.158 & $451 \pm 10$ & . & \\
\hline 2717 & 0.908 & -22.667 & -23.344 & -23.886 & $235 \pm 10$ & 16 & $\mathrm{C}$ \\
\hline 2731 & 0.510 & -22.790 & -23.151 & & $291 \pm 6$ & & \\
\hline 2734 & 0.864 & -22.893 & -23.454 & -23.884 & $236 \pm 15$ & 44 & $\mathrm{R}$ \\
\hline 2764 & 0.528 & -23.223 & -23.558 & -23.779 & $357 \pm 6$ & $\ldots$ & $\ldots$ \\
\hline 2771 & 0.563 & -22.862 & -23.261 & -23.652 & $263 \pm 5$ & $\ldots$ & $\ldots$ \\
\hline 2793 & 0.304 & -22.210 & & & $301 \pm 10$ & $\ldots$ & $\ldots$ \\
\hline 2799 & 0.533 & -22.619 & -22.930 & -23.060 & $271 \pm 13$ & $\ldots$ & $\ldots$ \\
\hline 2800 & 0.503 & -22.954 & & & $271 \pm 10$ & & \\
\hline 2806 & 0.351 & -22.664 & -22.885 & & $285 \pm 9$ & $\ldots$ & $\ldots$ \\
\hline 2810 & 0.451 & -22.553 & -22.845 & -22.998 & $265 \pm 12$ & $\ldots$ & $\ldots$ \\
\hline 2819 & 0.297 & -22.930 & -23.142 & $\ldots$ & $274 \pm 7$ & .. & $\ldots$ \\
\hline 2824 & 0.437 & -22.638 & -22.895 & $\ldots$ & $250 \pm 13$ & $\ldots$ & $\ldots$ \\
\hline 2836 & 0.325 & -22.409 & -22.596 & & & & \\
\hline 2841 & 0.554 & -22.952 & -23.333 & $\ldots$ & $265 \pm 10$ & $\ldots$ & $\ldots$ \\
\hline 2854 & 0.659 & -22.402 & -22.879 & 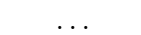 & $256 \pm 10$ & . & \\
\hline 2859 & 0.486 & -22.657 & & & $283 \pm 6$ & $\ldots$ & . \\
\hline 2864 & 0.488 & -22.325 & -22.645 & -22.920 & $240 \pm 6$ & $\cdots$ & \\
\hline 2870 & 0.590 & -22.736 & -23.129 & -23.396 & $292 \pm 16$ & & \\
\hline 2877 & 0.594 & -23.518 & -23.939 & $\ldots$ & $360 \pm 46$ & 69 & $\mathrm{R}$ \\
\hline 2881 & 0.390 & -22.292 & -22.530 & & $223 \pm 3$ & $\cdots$ & $\ldots$ \\
\hline 2896 & 0.353 & -22.548 & & & $238 \pm 7$ & $\ldots$ & $\ldots$ \\
\hline 2923 & 0.533 & -23.048 & -23.352 & -23.542 & $274 \pm 5$ & $\ldots$ & \\
\hline 2954 & 0.163 & -21.906 & -22.030 & & & $\ldots$ & \\
\hline 2992 & 0.340 & -22.569 & -22.799 & -23.046 & $257 \pm 7$ & $\ldots$ & $\ldots$ \\
\hline 3004 & 0.521 & -22.728 & -23.073 & & $223 \pm 8$ & & \\
\hline 3009 & 0.712 & -23.151 & -23.608 & -23.892 & $249 \pm 13$ & $\ldots$ & $\ldots$ \\
\hline 3027 & 0.622 & -22.752 & -23.191 & -23.561 & $237 \pm 6$ & & \\
\hline 3045 & 0.463 & -22.807 & -23.132 & $\ldots$ & $258 \pm 9$ & $\ldots$ & $\ldots$ \\
\hline 3074 & 0.732 & -23.134 & -23.612 & & $275 \pm 6$ & $\ldots$ & \\
\hline 3078 & 0.697 & -23.015 & -23.460 & -23.756 & $251 \pm 13$ & & \\
\hline 3089 & 0.524 & -22.563 & -22.843 & & $227 \pm 7$ & $\ldots$ & $\ldots$ \\
\hline 3094 & 0.571 & -22.914 & -23.258 & -23.452 & $266 \pm 11$ & & \\
\hline 3095 & 0.405 & -22.900 & -23.139 & & $303 \pm 6$ & $\ldots$ & $\ldots$ \\
\hline 3098 & 0.704 & -22.837 & -23.314 & -23.692 & $249 \pm 17$ & & \\
\hline 3100 & 0.533 & -22.485 & -22.833 & -23.093 & $208 \pm 13$ & $\ldots$ & $\ldots$ \\
\hline 3104 & 0.692 & -22.887 & -23.420 & -23.807 & $305 \pm 10$ & 18 & $\mathrm{R}$ \\
\hline 3106 & 0.778 & -22.780 & -23.364 & -23.859 & $242 \pm 5$ & & \\
\hline
\end{tabular}


Table 7 - Continued

\begin{tabular}{|c|c|c|c|c|c|c|c|}
\hline Abell & $\alpha$ & $M_{m}$ & $M_{m}\left(2 r_{m}\right)$ & $M_{m}\left(4 r_{m}\right)$ & $\begin{array}{c}\sigma \\
\mathrm{km} \mathrm{s}^{-1}\end{array}$ & $\begin{array}{c}r_{x} \\
\mathrm{kpc}\end{array}$ & Ref \\
\hline 3107 & 0.498 & -22.232 & -22.619 & & $240 \pm 14$ & & \\
\hline 3109 & 0.601 & -23.083 & -23.472 & -23.709 & $263 \pm 10$ & $\ldots$ & \\
\hline 3110 & 0.537 & -23.424 & -23.761 & -23.960 & $324 \pm 9$ & $\ldots$ & . \\
\hline 3111 & 0.717 & -23.063 & -23.562 & -23.948 & $311 \pm 13$ & & \\
\hline 3112 & 0.798 & -23.161 & -23.718 & -24.157 & $299 \pm 12$ & 0 & $\mathrm{C}$ \\
\hline 3120 & 0.744 & -22.797 & -23.312 & -23.704 & $270 \pm 7$ & $\ldots$ & $\ldots$ \\
\hline 3122 & 0.553 & -23.024 & -23.396 & -23.720 & $298 \pm 5$ & $\ldots$ & \\
\hline 3123 & 0.443 & -22.800 & -23.092 & & $345 \pm 5$ & $\ldots$ & $\ldots$ \\
\hline 3125 & 0.433 & -22.651 & -22.905 & -23.085 & $221 \pm 4$ & & \\
\hline 3128 & 0.578 & -23.121 & -23.507 & -23.766 & $269 \pm 5$ & 404 & $\mathrm{R}$ \\
\hline 3133 & 0.478 & -21.887 & -22.186 & -22.446 & $210 \pm 13$ & $\ldots$ & $\ldots$ \\
\hline 3135 & 0.449 & -22.822 & -23.092 & -23.244 & $283 \pm 5$ & $\ldots$ & $\ldots$ \\
\hline 3142 & 0.576 & -22.821 & -23.234 & & $241 \pm 6$ & $\ldots$ & $\ldots$ \\
\hline 3144 & 0.433 & -22.496 & -22.806 & -23.070 & $263 \pm 8$ & $\ldots$ & \\
\hline 3151 & 0.709 & -22.425 & -22.897 & & $288 \pm 18$ & & \\
\hline 3158 & 0.622 & -23.096 & -23.512 & -23.849 & $301 \pm 5$ & 458 & C \\
\hline 3164 & 0.513 & -23.108 & -23.452 & -23.726 & $306 \pm 5$ & 448 & $\mathrm{R}$ \\
\hline 3188 & 0.257 & -21.462 & & $\ldots$ & $217 \pm 8$ & $\ldots$ & $\ldots$ \\
\hline 3193 & 0.467 & -22.698 & -23.009 & & $278 \pm 9$ & $\ldots$ & \\
\hline 3195 & 0.585 & -22.833 & -23.167 & . & $261 \pm 14$ & $\ldots$ & $\ldots$ \\
\hline 3202 & 0.352 & -22.151 & & & $246 \pm 5$ & .. & \\
\hline 3223 & 0.478 & -23.143 & -23.474 & -23.714 & $333 \pm 11$ & $\ldots$ & ( \\
\hline 3225 & 0.617 & -22.518 & -22.948 & -23.361 & $218 \pm 5$ & $\ldots$ & $\ldots$ \\
\hline 3231 & 0.452 & -22.166 & -22.427 & & $188 \pm 6$ & $\ldots$ & \\
\hline 3266 & 0.845 & -23.329 & -23.957 & -24.504 & $257 \pm 11$ & 2 & $\mathrm{C}$ \\
\hline 3301 & 0.706 & -23.271 & -23.748 & -24.180 & $270 \pm 10$ & & \\
\hline 3323 & 0.845 & -22.435 & -23.057 & -23.471 & $244 \pm 8$ & $\ldots$ & $\ldots$ \\
\hline 3332 & 0.476 & -22.971 & -23.295 & -23.584 & $334 \pm 12$ & $\ldots$ & $\ldots$ \\
\hline 3336 & 0.711 & -22.975 & -23.480 & -23.894 & $261 \pm 13$ & $\ldots$ & \\
\hline 3341 & 0.657 & -22.585 & -23.042 & -23.395 & $258 \pm 10$ & 12 & $\mathrm{R}$ \\
\hline 3354 & 0.440 & -22.746 & -23.036 & -23.258 & & $\ldots$ & $\ldots$ \\
\hline 3367 & 0.375 & -22.393 & -22.654 & & $222 \pm 12$ & $\ldots$ & $\ldots$ \\
\hline 3374 & 0.272 & -22.593 & -22.655 & & $240 \pm 35$ & & \\
\hline 3376 & 0.622 & -23.037 & -23.466 & -23.871 & $319 \pm 9$ & 995 & $\mathrm{C}$ \\
\hline 3380 & 0.526 & -22.781 & -23.167 & -23.563 & $244 \pm 5$ & $\ldots$ & $\ldots$ \\
\hline 3381 & 0.584 & -22.399 & -22.817 & & $240 \pm 7$ & & \\
\hline 3389 & 0.520 & -23.084 & -23.428 & & $270 \pm 10$ & 81 & $\mathrm{R}$ \\
\hline 3390 & 0.581 & -22.530 & -22.931 & -23.266 & $264 \pm 3$ & 42 & $\mathrm{R}$ \\
\hline 3391 & 0.690 & -23.424 & -23.879 & & $320 \pm 6$ & 13 & $\mathrm{C}$ \\
\hline 3392 & 0.494 & -23.215 & -23.564 & -23.845 & $264 \pm 13$ & & \\
\hline 3395 & 0.781 & -22.946 & -23.467 & -23.806 & $287 \pm 23$ & 37 & $\mathrm{C}$ \\
\hline 3407 & 0.696 & -23.223 & -23.687 & -24.041 & $270 \pm 9$ & & \\
\hline 3408 & 0.592 & -23.363 & -23.780 & -24.138 & $297 \pm 4$ & 97 & $\mathrm{R}$ \\
\hline 3420 & 0.392 & -22.730 & -22.999 & & $260 \pm 12$ & $\ldots$ & $\ldots$ \\
\hline 3429 & 0.632 & -23.033 & -23.423 & -23.770 & $260 \pm 4$ & & \\
\hline 3432 & 0.472 & -22.869 & -23.194 & & $260 \pm 11$ & $\ldots$ & \\
\hline 3490 & 0.669 & -23.267 & -23.721 & -24.070 & $307 \pm 9$ & 84 & $\mathrm{R}$ \\
\hline 3492 & 0.421 & -22.765 & -23.042 & & & & \\
\hline 3494 & 0.848 & -22.267 & -22.821 & -23.299 & $185 \pm 26$ & & \\
\hline 3497 & 0.648 & -22.673 & -23.107 & -23.567 & $230 \pm 9$ & 352 & $\mathrm{R}$ \\
\hline 3500 & 0.311 & -22.389 & -22.582 & & $225 \pm 11$ & $\cdots$ & \\
\hline 3505 & 0.529 & -22.992 & -23.381 & & $247 \pm 13$ & & \\
\hline 3526 & 0.759 & -23.332 & -23.814 & & $288 \pm 11$ & 21 & $\mathrm{R}$ \\
\hline 3528 & 0.669 & -23.334 & -23.818 & -24.249 & $364 \pm 10$ & 7 & $\mathrm{R}$ \\
\hline 3530 & 0.765 & -23.270 & -23.849 & -24.355 & $292 \pm 14$ & 72 & $\mathrm{R}$ \\
\hline 3531 & 0.845 & -22.593 & -23.144 & & & & \\
\hline 3532 & 0.724 & -23.086 & -23.635 & -24.184 & $280 \pm 15$ & 128 & $\mathrm{R}$ \\
\hline 3537 & 0.527 & -23.057 & -23.410 & -23.769 & $321 \pm 11$ & $\ldots$ & \\
\hline 3542 & 0.279 & -22.450 & -22.637 & $\ldots$ & $303 \pm 9$ & & \\
\hline 3548 & 0.385 & -22.690 & -22.938 & . & $283 \pm 7$ & $\ldots$ & \\
\hline 3549 & 0.491 & -22.152 & & 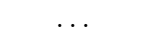 & $117 \pm 50$ & $\ldots$ & $\ldots$ \\
\hline 3552 & 0.571 & -22.731 & -23.119 & & $238 \pm 11$ & & \\
\hline 3553 & 0.444 & -22.452 & -22.731 & & $245 \pm 9$ & $\ldots$ & $\ldots$ \\
\hline 3554 & 0.725 & -22.858 & -23.340 & -23.732 & $263 \pm 11$ & & \\
\hline 3556 & 0.598 & -23.383 & -23.788 & & $347 \pm 12$ & 624 & $\mathrm{R}$ \\
\hline 3557 & 0.529 & -22.891 & -23.248 & -23.556 & & & \\
\hline 3558 & 0.906 & -23.451 & -24.061 & -24.561 & $282 \pm 14$ & 4 & $\mathrm{C}$ \\
\hline 3559 & 0.627 & -23.327 & -23.775 & -24.118 & $313 \pm 7$ & & \\
\hline 3560 & 0.331 & -22.488 & & & $234 \pm 7$ & 159 & $\mathrm{R}$ \\
\hline 3562 & 0.703 & -22.995 & -23.511 & -24.012 & $263 \pm 6$ & 38 & $\mathrm{C}$ \\
\hline 3563 & 0.362 & -22.527 & -22.759 & & $248 \pm 13$ & & \\
\hline 3564 & 0.457 & -22.826 & -23.096 & -23.197 & $276 \pm 5$ & .. & \\
\hline 3565 & 0.551 & -23.224 & -23.613 & - & $883 \pm 29$ & .. & \\
\hline 3570 & 0.330 & -22.678 & -22.873 & & $284 \pm 20$ & & \\
\hline 3571 & 1.125 & -23.280 & -24.003 & -24.496 & $313 \pm 11$ & 0 & $\mathrm{C}$ \\
\hline 3572 & 0.353 & -22.712 & -22.932 & & $239 \pm 2$ & $\ldots$ & \\
\hline
\end{tabular}


Present Epoch Brightest Cluster Galaxies

Table 7 - Continued

\begin{tabular}{|c|c|c|c|c|c|c|c|}
\hline Abell & $\alpha$ & $M_{m}$ & $M_{m}\left(2 r_{m}\right)$ & $M_{m}\left(4 r_{m}\right)$ & $\begin{array}{c}\sigma \\
\mathrm{km} \mathrm{s}^{-1}\end{array}$ & $\begin{array}{c}r_{x} \\
\mathrm{kpc}\end{array}$ & Ref \\
\hline 3574 & 0.743 & -23.064 & -23.588 & & $239 \pm 11$ & $\ldots$ & $\cdots$ \\
\hline 3575 & 0.280 & -21.949 & -22.168 & & $283 \pm 14$ & & $\cdots$ \\
\hline 3577 & 0.542 & -22.552 & & & $297 \pm 5$ & $\cdots$ & \\
\hline 3581 & 0.638 & -22.642 & -23.073 & -23.588 & $283 \pm 3$ & $\ddot{1}$ & $\ddot{\mathrm{C}}$ \\
\hline 3599 & 0.160 & -21.329 & & $\ldots$ & $223 \pm 5$ & $\ldots$ & $\ldots$ \\
\hline 3603 & 0.494 & -22.996 & -23.326 & & & $\ldots$ & $\ldots$ \\
\hline 3605 & 0.675 & -22.933 & -23.390 & -23.795 & $276 \pm 12$ & $\ldots$ & $\ldots$ \\
\hline 3615 & 0.579 & -23.236 & -23.647 & & $279 \pm 20$ & $\ldots$ & $\ldots$ \\
\hline 3651 & 0.730 & -23.302 & -23.757 & -24.049 & $284 \pm 15$ & $\cdots$ & \\
\hline 3656 & 0.574 & -23.001 & -23.444 & -23.948 & $296 \pm 3$ & & \\
\hline 3667 & 0.768 & -23.320 & -23.843 & -24.261 & $327 \pm 13$ & 137 & $\mathrm{C}$ \\
\hline 3676 & 0.468 & -22.872 & -23.164 & $\ldots$ & $218 \pm 29$ & $\ldots$ & $\ldots$ \\
\hline 3677 & 0.421 & -22.135 & -22.462 & $\ldots$ & $223 \pm 16$ & $\ldots$ & $\ldots$ \\
\hline 3684 & 0.496 & -22.483 & -22.819 & $\cdots$ & $294 \pm 13$ & $\ldots$ & $\ldots$ \\
\hline 3685 & 0.068 & -21.200 & & & $96 \pm 25$ & $\ldots$ & $\ldots$ \\
\hline 3687 & 0.672 & -22.959 & -23.403 & -23.727 & $251 \pm 9$ & . & $\cdots$ \\
\hline 3698 & 0.366 & -22.298 & & & $285 \pm 4$ & $\ldots$ & . \\
\hline 3703 & 0.610 & -22.942 & -23.396 & -23.771 & $234 \pm 7$ & & \\
\hline 3716 & 0.719 & -22.988 & -23.483 & -23.842 & $275 \pm 10$ & 271 & $\mathrm{R}$ \\
\hline 3731 & 0.497 & -22.950 & -23.296 & 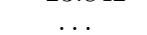 & $276 \pm 5$ & & \\
\hline 3733 & 0.632 & -22.561 & -23.017 & & $314 \pm 12$ & 182 & $\mathrm{R}$ \\
\hline 3736 & 0.679 & -23.301 & -23.752 & -24.081 & $282 \pm 9$ & $\ldots$ & $\ldots$ \\
\hline 3741 & 0.670 & -22.857 & -23.322 & -23.723 & $229 \pm 5$ & $\ldots$ & $\cdots$ \\
\hline 3742 & 0.415 & -22.376 & -22.642 & $\ldots$ & $287 \pm 8$ & $\ldots$ & $\ldots$ \\
\hline 3744 & 0.442 & -22.901 & -23.190 & $\ldots$ & $287 \pm 8$ & $\ldots$ & $\ldots$ \\
\hline 3747 & 0.436 & -22.631 & -22.916 & & $254 \pm 12$ & $\ldots$ & $\ldots$ \\
\hline 3753 & 0.169 & -21.916 & & & $141 \pm 37$ & $\ldots$ & $\ldots$ \\
\hline 3764 & 0.463 & -22.861 & -23.197 & & $215 \pm 6$ & $\ldots$ & $\ldots$ \\
\hline 3771 & 0.612 & -22.853 & -23.240 & -23.573 & $237 \pm 5$ & $\cdots$ & $\cdots$ \\
\hline 3781 & 0.501 & -21.893 & -22.182 & & & $\cdots$ & $\cdots$ \\
\hline 3782 & 0.619 & -23.088 & -23.506 & -23.854 & $259 \pm 5$ & $\ldots$ & $\ldots$ \\
\hline 3785 & 0.541 & -23.335 & -23.704 & -23.994 & $366 \pm 6$ & $\ldots$ & $\ldots$ \\
\hline 3796 & 0.610 & -22.770 & -23.214 & -23.613 & $230 \pm 7$ & .. & . \\
\hline 3799 & 0.471 & -22.211 & -22.553 & -22.855 & $268 \pm 6$ & $\ldots$ & \\
\hline 3806 & 0.772 & -23.346 & -23.848 & -24.260 & $277 \pm 0$ & 51 & $\ddot{R}$ \\
\hline 3809 & 0.720 & -22.822 & -23.319 & -23.721 & $246 \pm 6$ & 116 & $\mathrm{R}$ \\
\hline 3816 & 0.544 & -22.622 & -23.009 & -23.353 & $258 \pm 5$ & $\ldots$ & $\ldots$ \\
\hline 3822 & 0.628 & -23.052 & -23.455 & -23.777 & $328 \pm 6$ & & \\
\hline 3825 & 0.448 & -22.916 & -23.216 & -23.477 & & 1064 & $\mathrm{R}$ \\
\hline 3826 & 0.712 & -23.313 & -23.758 & -24.093 & $272 \pm 6$ & $\ldots$ & $\ldots$ \\
\hline 3844 & 0.366 & -21.851 & -22.157 & & $215 \pm 17$ & $\ldots$ & $\cdots$ \\
\hline 3851 & 0.508 & -22.815 & -23.163 & -23.471 & $239 \pm 10$ & $\ldots$ & $\ldots$ \\
\hline 3869 & 0.379 & -22.736 & -22.981 & & $284 \pm 13$ & $\ldots$ & $\ldots$ \\
\hline 3879 & 0.484 & -22.624 & -22.931 & -23.169 & $228 \pm 14$ & $\ldots$ & $\ldots$ \\
\hline 3880 & 0.751 & -22.936 & -23.403 & -23.716 & $251 \pm 13$ & 31 & $\mathrm{R}$ \\
\hline 3895 & 0.393 & -22.798 & -23.024 & & $295 \pm 4$ & $\ldots$ & $\ldots$ \\
\hline 3897 & 0.567 & -23.127 & -23.555 & -23.924 & $341 \pm 13$ & $\because$ & $\therefore$ \\
\hline 3898 & 0.410 & -22.011 & & & $212 \pm 14$ & $\cdots$ & $\cdots$ \\
\hline 3912 & 0.678 & -22.807 & -23.217 & -23.513 & $245 \pm 19$ & $\ldots$ & $\ldots$ \\
\hline 3925 & 0.554 & -23.144 & -23.560 & -23.914 & & .. & \\
\hline 4008 & 0.591 & -22.993 & -23.366 & -23.694 & $270 \pm 11$ & & $\ldots$ \\
\hline 4038 & 0.449 & -22.706 & -22.993 & & $274 \pm 8$ & 137 & $\mathrm{C}$ \\
\hline 4049 & 0.512 & -22.848 & -23.239 & & $272 \pm 9$ & & \\
\hline 4059 & 0.891 & -23.246 & -23.841 & -24.287 & $289 \pm 8$ & 8 & C \\
\hline
\end{tabular}

Note. - Columns: (1) Abell cluster number, (2) $\alpha$ at the metric radius, (3) absolute metric luminosity, (4) absolute metric luminosity within $2 r_{m}$, (5) absolute metric luminosity within $4 r_{m}$, (6) galaxy stellar velocity dispersion, (7) radial offset of the BCG from the X-ray cluster center, (8) X-ray reference, $\mathrm{R}=\mathrm{ROSAT}, \mathrm{C}=\mathrm{Chandra}$. The $\mathrm{CMB}$ frame has been adopted for the calculation of metric luminosities. 
LAUER ET AL.

Table 8

M2 Parameters

\begin{tabular}{|c|c|c|c|c|}
\hline Abell & $\alpha$ & $M_{m}$ & $\begin{array}{c}\sigma \\
\mathrm{km} \mathrm{s}^{-1}\end{array}$ & $\begin{array}{c}r_{x} \\
\mathrm{kpc}\end{array}$ \\
\hline 0027 & 0.542 & -22.229 & $224 \pm 20$ & \\
\hline 0071 & 0.323 & -22.340 & $223 \pm 21$ & $\ldots$ \\
\hline 0074 & 0.408 & -22.669 & $\ldots$ & \\
\hline 0075 & 0.528 & -22.316 & $\ldots$ & \\
\hline 0076 & 0.397 & -22.601 & & 313 \\
\hline 0086 & 0.469 & -22.864 & $260 \pm 16$ & 235 \\
\hline 0102 & 0.549 & -22.580 & & \\
\hline 0116 & 0.473 & -22.513 & $\ldots$ & 429 \\
\hline 0119 & 0.479 & -22.993 & $\ldots$ & 139 \\
\hline 0134 & 0.476 & -21.550 & $\ldots$ & 1307 \\
\hline 0147 & 0.570 & -22.426 & $\ldots$ & 44 \\
\hline 0154 & 0.677 & -23.026 & $\ldots$ & 141 \\
\hline 0161 & 0.168 & -22.143 & & \\
\hline 0171 & 0.410 & -22.722 & $\ldots$ & $\ldots$ \\
\hline 0174 & 0.403 & -22.497 & $\ldots$ & . \\
\hline 0179 & 0.584 & -22.403 & $\ldots$ & $\ldots$ \\
\hline 0240 & 0.338 & -22.547 & $\ldots$ & \\
\hline 0246 & 0.351 & -22.306 & $\ldots$ & 116 \\
\hline 0399 & 0.417 & -23.109 & $\ldots$ & 398 \\
\hline 0400 & 0.181 & -22.102 & & 4 \\
\hline 0428 & 0.405 & -22.251 & $200 \pm 24$ & . \\
\hline 0436 & 0.495 & -22.331 & $202 \pm 45$ & \\
\hline 0500 & 0.550 & -23.049 & $\ldots$ & 971 \\
\hline 0533 & 0.521 & -22.724 & $\ldots$ & \\
\hline 0539 & 0.791 & -22.523 & $\ldots$ & 2 \\
\hline 0568 & 0.340 & -22.488 & $\ldots$ & \\
\hline 0576 & 0.548 & -22.334 & $\ldots$ & 43 \\
\hline 0582 & 0.324 & -22.600 & & $\ldots$ \\
\hline 0592 & 0.314 & -22.829 & $267 \pm 9$ & \\
\hline 0595 & 0.489 & -22.647 & $281 \pm 17$ & 360 \\
\hline 0602 & 0.810 & -22.300 & $\ldots$ & 58 \\
\hline 0819 & 0.469 & -22.414 & & \\
\hline 0838 & 0.409 & -22.366 & $164 \pm 10$ & $\ldots$ \\
\hline 0841 & 0.570 & -22.742 & $237 \pm 11$ & \\
\hline 0970 & 0.307 & -22.227 & $\ldots$ & 81 \\
\hline 0978 & 0.321 & -22.307 & $\ldots$ & 257 \\
\hline 0979 & 0.417 & -22.375 & $\ldots$ & \\
\hline 1032 & 0.356 & -22.528 & $\ldots$ & 645 \\
\hline 1066 & 0.531 & -22.695 & $\ldots$ & \\
\hline 1069 & 0.412 & -22.033 & $\ldots$ & 563 \\
\hline 1142 & 0.664 & -22.618 & $\ldots$ & 139 \\
\hline 1145 & 0.272 & -22.110 & $\ldots$ & $\ldots$ \\
\hline 1149 & 0.376 & -22.633 & $\ldots$ & \\
\hline 1169 & 0.320 & -22.180 & $\ldots$ & 163 \\
\hline 1171 & 0.523 & -22.448 & $\ldots$ & 260 \\
\hline 1187 & 0.414 & -22.554 & $\ldots$ & 641 \\
\hline 1190 & 0.551 & -22.929 & $\ldots$ & $\ldots$ \\
\hline 1203 & 0.337 & -22.283 & $\ldots$ & \\
\hline 1228 & 0.533 & -22.281 & $\ldots$ & 277 \\
\hline 1238 & 0.404 & -22.412 & $\ldots$ & \\
\hline 1317 & 0.429 & -22.424 & & 603 \\
\hline 1318 & 0.653 & -22.763 & $235 \pm 14$ & 922 \\
\hline 1371 & 0.591 & -22.734 & & 291 \\
\hline 1375 & 0.642 & -23.118 & $298 \pm 6$ & \\
\hline 1377 & 0.565 & -22.691 & $\ldots$ & 738 \\
\hline 1383 & 0.487 & -22.450 & & 458 \\
\hline 1436 & 0.506 & -22.583 & $238 \pm 12$ & 303 \\
\hline 1452 & 0.562 & -22.857 & $\ldots$ & \\
\hline 1630 & 0.491 & -22.944 & $\ldots$ & 718 \\
\hline 1644 & 0.566 & -22.802 & & 726 \\
\hline 1648 & 0.460 & -22.331 & $256 \pm 12$ & \\
\hline 1656 & 0.859 & -22.984 & $\ldots$ & 103 \\
\hline 1709 & 0.571 & -22.420 & & \\
\hline 1736 & 0.636 & -22.850 & $\ldots$ & 66 \\
\hline 1767 & 0.330 & -22.799 & $\ldots$ & 445 \\
\hline 1773 & 0.298 & -22.496 & $\ldots$ & 536 \\
\hline 1775 & 0.439 & -23.089 & & 61 \\
\hline 1780 & 0.661 & -22.808 & $313 \pm 17$ & 113 \\
\hline 1809 & 0.702 & -22.772 & $\ldots$ & 340 \\
\hline 1825 & 0.504 & -22.850 & & \\
\hline 1827 & 0.550 & -22.310 & $273 \pm 21$ & \\
\hline 1890 & 0.534 & -22.598 & $240 \pm 20$ & 306 \\
\hline 1898 & 0.367 & -22.730 & $\ldots$ & 215 \\
\hline 1982 & 0.302 & -21.777 & $\ldots$ & \\
\hline 1983 & 0.786 & -22.250 & $\ldots$ & 64 \\
\hline
\end{tabular}


Present Epoch Brightest Cluster Galaxies

Table 8 - Continued

\begin{tabular}{|c|c|c|c|c|}
\hline Abell & $\alpha$ & $M_{m}$ & $\begin{array}{c}\sigma \\
\mathrm{km} \mathrm{s}^{-1}\end{array}$ & $\begin{array}{c}r_{x} \\
\mathrm{kpc}\end{array}$ \\
\hline 2022 & 0.298 & -21.818 & . & 254 \\
\hline 2028 & 0.394 & -22.359 & $\ldots$ & \\
\hline 2061 & 0.493 & -22.989 & $\ldots$ & 623 \\
\hline 2065 & 0.803 & -22.420 & $\cdots$ & 8 \\
\hline 2079 & 0.241 & -22.557 & $\ldots$ & 233 \\
\hline 2147 & 0.571 & -22.632 & $\ldots$ & 183 \\
\hline 2152 & 0.382 & -22.369 & $\ldots$ & 109 \\
\hline 2197 & 0.696 & -22.791 & $\ldots$ & 27 \\
\hline 2198 & 0.038 & -20.905 & $\ldots$ & $\ldots$ \\
\hline 2241 & 0.362 & -22.341 & $\ldots$ & \\
\hline 2247 & 0.641 & -22.460 & $\ldots$ & \\
\hline 2248 & 0.485 & -22.643 & & 1493 \\
\hline 2250 & 0.344 & -22.694 & $275 \pm 16$ & \\
\hline 2256 & 0.404 & -23.143 & $311 \pm 9$ & 2446 \\
\hline 2293 & 0.600 & -22.621 & $282 \pm 15$ & \\
\hline 2325 & 0.379 & -22.452 & $206 \pm 11$ & \\
\hline 2331 & 0.429 & -22.405 & & 552 \\
\hline 2361 & 0.435 & -22.759 & $301 \pm 12$ & $\ldots$ \\
\hline 2370 & 0.167 & -21.716 & $\ldots$ & \\
\hline 2372 & 0.377 & -22.320 & $\ldots$ & \\
\hline 2382 & 0.444 & -22.781 & $\ldots$ & 376 \\
\hline 2399 & 0.408 & -22.712 & $\ldots$ & 309 \\
\hline 2412 & 0.277 & -21.764 & $\ldots$ & 710 \\
\hline 2459 & 0.423 & -22.786 & $\ldots$ & \\
\hline 2462 & 0.281 & -21.958 & $\ldots$ & 149 \\
\hline 2492 & 0.404 & -22.112 & $\ldots$ & 302 \\
\hline 2511 & 0.586 & -22.353 & $\ldots$ & $\ldots$ \\
\hline 2524 & 0.594 & -22.980 & $\ldots$ & $\ldots$ \\
\hline 2525 & 0.354 & -22.515 & $\ldots$ & \\
\hline 2572 & 0.671 & -22.434 & & 32 \\
\hline 2618 & 0.659 & -23.255 & $332 \pm 11$ & \\
\hline 2625 & 0.525 & -22.827 & & 1125 \\
\hline 2637 & 0.342 & -22.615 & $312 \pm 11$ & 467 \\
\hline 2656 & 0.200 & -21.633 & $\ldots$ & \\
\hline 2657 & 0.890 & -22.354 & $\ldots$ & 1 \\
\hline 2678 & 0.363 & -22.641 & $\ldots$ & . \\
\hline 2764 & 0.400 & -22.436 & $\ldots$ & $\ldots$ \\
\hline 2771 & 0.353 & -22.154 & & $\ldots$ \\
\hline 2793 & 0.267 & -22.059 & $196 \pm 69$ & $\ldots$ \\
\hline 2799 & 0.423 & -21.934 & $\ldots$ & $\ldots$ \\
\hline 2800 & 0.478 & -22.773 & & \\
\hline 2806 & 0.627 & -22.404 & $207 \pm 12$ & $\ldots$ \\
\hline 2810 & 0.422 & -22.133 & $234 \pm 13$ & \\
\hline 2819 & 0.430 & -22.868 & $269 \pm 8$ & $\ldots$ \\
\hline 2824 & 0.371 & -22.198 & $231 \pm 20$ & $\ldots$ \\
\hline 2836 & 0.439 & -22.307 & $229 \pm 13$ & $\ldots$ \\
\hline 2854 & 0.361 & -22.307 & $221 \pm 20$ & $\ldots$ \\
\hline 2859 & 0.355 & -22.255 & $\ldots$ & $\ldots$ \\
\hline 2864 & 0.402 & -22.129 & $\ldots$ & $\ldots$ \\
\hline 2881 & 0.405 & -22.262 & $\ldots$ & $\ldots$ \\
\hline 2923 & 0.307 & -22.295 & $\ldots$ & $\ldots$ \\
\hline 2954 & 0.339 & -21.274 & $\ldots$ & $\ldots$ \\
\hline 3004 & 0.284 & -22.370 & $\ldots$ & $\ldots$ \\
\hline 3027 & 0.543 & -22.733 & $\ldots$ & $\ldots$ \\
\hline 3074 & 0.360 & -22.575 & $\ldots$ & \\
\hline 3078 & 0.256 & -22.071 & $\ldots$ & $\ldots$ \\
\hline 3089 & 0.531 & -22.416 & $\ldots$ & \\
\hline 3094 & 0.749 & -22.423 & & \\
\hline 3098 & 0.575 & -22.576 & $235 \pm 10$ & \\
\hline 3104 & 0.274 & -21.848 & $\ldots$ & 376 \\
\hline 3106 & 0.509 & -22.626 & $\ldots$ & $\ldots$ \\
\hline 3107 & 0.369 & -22.054 & $\ldots$ & \\
\hline 3109 & 0.290 & -21.348 & $\ldots$ & $\ldots$ \\
\hline 3110 & 0.468 & -22.752 & $\ldots$ & $\ldots$ \\
\hline 3120 & 0.305 & -21.472 & & \\
\hline 3122 & 0.611 & -22.761 & $274 \pm 11$ & $\ldots$ \\
\hline 3123 & 0.249 & -22.009 & & \\
\hline 3128 & 0.541 & -22.898 & $\ldots$ & 1057 \\
\hline 3133 & 0.181 & -21.340 & & \\
\hline 3135 & 0.510 & -22.778 & & $\ldots$ \\
\hline 3151 & 0.610 & -22.304 & $229 \pm 25$ & \\
\hline 3158 & 0.723 & -22.987 & & 56 \\
\hline 3164 & 0.397 & -22.947 & & 1109 \\
\hline 3223 & 0.311 & -22.561 & $233=$ & \\
\hline 3341 & 0.222 & -22.352 & $\ldots$ & 130 \\
\hline 3354 & 0.418 & -22.455 & $\ldots$ & \\
\hline
\end{tabular}


Table 8 - Continued

\begin{tabular}{ccclc}
\hline \hline & & & \multicolumn{2}{c}{$\sigma$} \\
Abell & $\alpha$ & $M_{m}$ & $\mathrm{~km} \mathrm{~s}^{-1}$ & \multicolumn{1}{c}{$\mathrm{kpc}$} \\
\hline 3374 & 0.376 & -22.397 & $\ldots$ & $\ldots$ \\
3389 & 0.506 & -22.909 & $293 \pm 9$ & 814 \\
3391 & 0.656 & -22.818 & $268 \pm 6$ & 80 \\
3408 & 0.666 & -22.849 & $245 \pm 5$ & 222 \\
3420 & 0.295 & -22.167 & $\ldots$ & $\ldots$ \\
3492 & 0.323 & -21.861 & $\ldots$ & $\ldots$ \\
3497 & 0.296 & -22.449 & $\ldots$ & 400 \\
3505 & 0.357 & -22.767 & $296 \pm 11$ & $\ldots$ \\
3531 & 0.553 & -22.645 & $\ldots$ & $\ldots$ \\
3548 & 0.500 & -22.607 & $\ldots$ & $\ldots$ \\
3570 & 0.633 & -22.211 & $\ldots$ & $\ldots$ \\
3577 & 0.422 & -22.417 & $199 \pm 5$ & $\ldots$ \\
3603 & 0.544 & -22.162 & $106 \pm 3$ & $\ldots$ \\
3605 & 0.368 & -22.711 & $\ldots$ & $\ldots$ \\
3651 & 0.336 & -22.580 & $\ldots$ & $\ldots$ \\
3733 & 0.506 & -22.553 & $279 \pm 20$ & 110 \\
3764 & 0.340 & -22.741 & $238 \pm 6$ & $\ldots$ \\
3771 & 0.373 & -22.818 & $\ldots$ & $\ldots$ \\
3785 & 0.540 & -23.281 & $\ldots$ & $\ldots$ \\
3799 & 0.305 & -21.702 & $161 \pm 8$ & $\ldots$ \\
3822 & 0.682 & -23.005 & $286 \pm 8$ & $\ldots$ \\
3825 & 0.535 & -22.792 & $249 \pm 6$ & 385 \\
3844 & 0.287 & -21.409 & $\ldots$ & $\ldots$ \\
3880 & 0.247 & -22.063 & $\ldots$ & 63 \\
3895 & 0.676 & -22.578 & $190 \pm 8$ & $\ldots$ \\
3912 & 0.379 & -22.325 & $\ldots$ & $\ldots$ \\
3925 & 0.394 & -22.401 & $\ldots$ & $\ldots$ \\
4038 & 0.719 & -22.499 & $249 \pm 9$ & 17 \\
& & & & \\
\hline & & & &
\end{tabular}

Note. - Columns: (1) Abell cluster number, (2) $\alpha$ at the metric radius, (3) absolute metric luminosity, (4) galaxy stellar velocity dispersion, (5) radial offset of the BCG from the X-ray cluster center, The CMB frame has been adopted for the calculation of metric luminosities. 